RC. $858-$ B

GEOLOGICAL SURVEY CIRCULAR 858-B

\title{
Preliminary Metallogenic Map of North America: An Alphabetical Listing of Deposits
}





\section{Preliminary Metallogenic Map of North America: An Alphabetical Listing of Deposits}

By Philip W. Guild

GEOLOGICAL SURVEY CIRCULAR 858-B

The names of 4,215 deposits are arranged in alphabetical order by political units (countries and subdivisions) 
United States Department of the Interior

JAMES G. WATT, Secretary

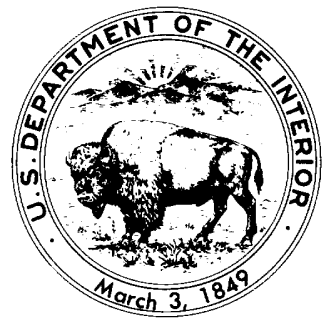

Geological Survey

Dallas L. Peck, Director

Library of Congress catalog-card No. 81-600157 


\section{CONTENTS}

Abstract

Introduction

Alphabetical listing of deposits

(The letters following names are the codes.)

Belize

British

\begin{tabular}{|c|c|}
\hline Alberta & CAAL \\
\hline British Columbia. & $\mathrm{CABC}$ \\
\hline Manitoba & CAMB \\
\hline New Brunswick & CANB. \\
\hline Newfoundland and Labrador & CANF \\
\hline Nova Scotia & CANS \\
\hline Ontario _. & $\mathrm{CAON}_{\text {- }}$ \\
\hline Quebec & CAQB \\
\hline Saskatchewan & CASA \\
\hline
\end{tabular}

\section{Northwest Territories}

Franklin District

Keewatin District

Mackenzie District

Yukon Territory

Costa Rica

Cuba

Dominican Republic

El Salvador

Greenland

Guatemala

Haiti

Honduras

Jamaica

Mexico

Aguascalientes

Baja California Norte

Baja California Sur

Chiapas

Chihuahua

Coahuila

Colima

Durango

Estado de México

Guanajuato

Guerrero

Hidalgo

Jalisco

Michoacán

Nayarit

Nuevo León

Oaxaca

Puebla

Querétaro

San Luis Potosí

Sinaloa

Sonora

Tamaulipas
UKUK _...--- 44

BHBH …-.. 2

VIVI _-.------ 72

2

2

2

8

11

11

12

13

17

21

8

8

8

9

22

24

24

25

25

25

26

26

26

26

27

27

27

27

32

28

31

31

32

34

34

34

35

35

36

37

37

38

39

40

42

40

40

42
Alphabetical listing of deposits-Continued Mexico-Continued

\begin{tabular}{|c|c|c|}
\hline Vera Cruz & MXVR & B42 \\
\hline Zacatecas & MXZC & 42 \\
\hline Nicaragua & NUNU & \\
\hline Panama.- & PNPN & \\
\hline Puerto Rico & RQRQ & \\
\hline Saint Pierre and Miquelon. & FRFR & \\
\hline United States & & \\
\hline Alabama & USAL & \\
\hline Alaska & USAK & \\
\hline Arizona & USAZ & \\
\hline Arkansas & USAR & \\
\hline California_-- & USCA & \\
\hline Colorado & USCO & \\
\hline Connecticut_. & USCT & \\
\hline Florida & USFL & \\
\hline Georgia & USGA & \\
\hline Idaho & USID_ & \\
\hline Illinois & USIL $_{-}$ & \\
\hline Indiana & USIN & \\
\hline Iowa & USIA & \\
\hline Kansas_.---. & USKS & \\
\hline Kentucky --- & USKY & \\
\hline Louisiana & USLA & \\
\hline Maine & USME. & \\
\hline Maryland _... & USMD & \\
\hline Massachusetts & USMA & \\
\hline Michigan .... & USMI & \\
\hline Minnesota & USMN & \\
\hline Mississippi _- & USMS & \\
\hline Missouri & USMO_ & \\
\hline Montana & USMT & \\
\hline Nevada & USNV & 52 \\
\hline New Hampshire & USNH & \\
\hline New Jersey & USNJ & \\
\hline New Mexico & USNM & \\
\hline New York & USNY. & \\
\hline North Carolina & USNC & \\
\hline North Dakota & USND_ & \\
\hline Ohio & USOH & 00 \\
\hline Oklahoma & USOK & 6 \\
\hline Oregon & USOR & be \\
\hline Pennsylvania. & USPA & 0 \\
\hline Rhode Island & USRI & $6^{r}$ \\
\hline South Carolina & USSC & 6 \\
\hline South Dakota & USSD & 6 \\
\hline Tennessee & USTN & 6 \\
\hline Texas & USTX . & 6 \\
\hline Utah & USUT & 6 \\
\hline Vermont & USVT & 7 \\
\hline Virginia & USVA & 7 \\
\hline Washington & USWA & 7 \\
\hline West Virginia & USWV & 71 \\
\hline Wisconsin & USWI & 7 \\
\hline Wyoming -- & USWY --- & \\
\hline
\end{tabular}

Page 42 



\title{
PRELIMINARY METALLOGENIC MAP OF NORTH AMERICA An Alphabetical Listing of Deposits
}

\author{
By Phillp W. Guild
}

ABSTRACT

The names of 4,215 ore deposits shown on the Preliminary Metallogenic Map of North America are listed in alphabetical order by country, and by subdivisions of the larger countries (Canada, Mexico, and the United States). Map numbers, major and minor constituents, geographic coordinates, and a geologic code are given for each entry.

\section{INTRODUCTION}

In Circular 858-A, the 4,215 deposits shown on the Preliminary Metallogenic Map of North America are identified by name, metal/mineral content, location, and certain other characteristics in a listing arranged numerically by country and by State, Province, or other subdivisions in the larger countries (Canada, Mexico, and the United States). Because many of these political units have from 100 to more than 300 individual entries, a listing arranged alphabetically by name within the units is provided in this Circular to aid the user in searching for a particular deposit that may be of interest to him. (The decision to publish these tables separately was made to avoid the problems in binding and handling that would be entailed in combining the two tables in a single volume.)

The explanatory information contained in Circular 858-A has not been repeated here; the user should refer to it for background in interpreting the entries in this Circular. This alphabetical listing differs in two respects from the numerical listing in Circular 858-A:

(1) Column headings are not repeated; the columns are, from left to right, a four-letter code identifying the country and subdivision, the map number, name, major and minor constituents, latitude and longitude, and the code.

(2) The order of the listing is alphabetical by the country/subdivision code, and by the initial letter in the deposit name. The country/subdivision code is given in the Contents; note that the order of this code does not necessarily follow in strict alphabetical order the names of the units to which they refer. 
METALLOGENIC MAP OF NORTH AMERICA

ALPHABETIC LISTING BY COUNTRY BY STATE BY DEPOSIT NAME

COUNTRY/

STITE

AL.-No.

NAME

MINOR

COORDINATES

BHBH 2 FIRST CREEK

BHBH 1 MOUNTAIN PINE RIDGE

BHBH 3 QUEBRADA CEIBO GRANDE

CAAL 11 DUNGARVAN CREEK

CAAL 8 DUVERNAY

CAAL $\quad 4$

MA JOR
BA
SN

LINDBERGH

MET I SKOW

$\begin{array}{lrl}\text { CAAL } & 9 & \text { METISKOW } \\ \text { CAAL } & 10 & \text { MOUNT INVINCIBLE }\end{array}$

CAAL 10 MOUNT INVINCIBLE

CAAL 6 NORTH SASKATCHEWAN RIVER

CAAL 1 PEACE POINT REGI

CAAL 5 STARLIGHT RANGE

CAAL 2 SWIFT CREEK

$\begin{array}{lrl}\text { CAAL } & 3 & \text { WATERWAYS } \\ \text { CABC } & 234 & \text { ADAMS PLATEAU AREA }\end{array}$

CABC 9 ADANAC-ADERA (RUBY CREEK AREA)

CABC 217 AFTON AND IRON MASK AREA

CABC 74 AIKEN LAKE (CROYDON)

CABC 82 AJAX

CABC 72 AKIE RIVER (ELF)

CABC 252 AKOLKOLEX RIVER (WIGWAM)

ALICE ARM AG AREA (DOLLY VARDEN, TORBRITT, ETC)

ALICE ARM MOLY AREA (B.C., ROUNDY CREEK, BELL)

ALSEK RIVER (WINOY)

$\begin{array}{lll}\text { CABC } & 2 & \\ \text { CABC ANKWILL CREEK (RAINBOW) }\end{array}$

CABC 206 ANTICLIMAX-ROCK ISLAND AREA

CABC 83 ANYOX (HIDDEN CREEK AND BONANZA MINE)

CABC 140 APEX

CABC 269 ARGONAUT MINE

CABC 282 ASHLOO MINE

CABC 65 ASITKA PEAK (ASITKA)

CABC 289 ASPEN GROVE AREA

CABC 111 ASTLAIS MOUNTAIN (BIG ONION)

CABC 26 ATAN LAKE-MOUNT HASKIN AREA

CABC 11 ATLIN AREA

CABC 96 ATNA RANGE

CABC 53 BALL CREEK AREA

CABC 117 BARRETT LAKE-EMERSON CREEK AREA (BARR, ETC)

CABC 47 BARRINGTON RIVER AREA

CABC 214 BASQUE

CABC 336

CABC 31

CABC 174

CABC 148

323

CABC 215

CABC 215

CABC 179

CABC 279

\begin{tabular}{ll}
$C A B C$ & 279 \\
\hline 2
\end{tabular}

BAYONNE MINE

BA

AU

FE
HAL

HAL

HAL

GYP

AU

GYP

FE

PB ZN AG

CU FE

CU AU MO

MO

$P B \quad Z N$ BA

$Z N$ PB

$A G P B Z N$ W $S N$

$A G P B$

MO

$\mathrm{CU}$

CU

CU FES

FE

AU CU

$\mathrm{CU}$

$\mathrm{CU}$

CU MO

ZN

AU

CU MO

MO $\mathrm{CU}$

MO

BEAVERDELL AREA (HIGHLAND-BELL MINE, ETC.)

AU AG

作

CU FE AU

CU MO

BERLIN, INLAND EMPIRE, ALICE LAKE

AU AG

BETHLEHEM MINE VALLEY, ETC.

BIRCH ISLAND (REXSPAR)

U $F$

SR TH

AU

BLUE GROUSE MINE

BLUEBELL MINE

ZN PB AG CD

N16 50 LONG. CODE LONG.

W088 $55 \quad 183 C 4 A 8$

N16 35 WOB9 10 4A3C4A8

N49 12 W113 55 2A1C4A5

N53 47 W11141 GB37A6A3

45346

N53 53

N52 21

N5O 40

N53 45

N59 10

N53 27

N56 52

N56 42

N51 03

N59 42

$\begin{array}{lll}\text { N5O } & 39 \\ \text { N56 } & 27\end{array}$

N55 35

N57 18

N5O 52

N51 12

N55 42

N55 25

N59 45

N55 40

N51 36

N55 26

N52 42

N49 52

N49 57

N56 37

N49 55

N54 49

N59 16

N59 33

N55 52

N57 15

N54 26

N57 53

N50 36

N49 07

N49 26

N5O 23

N53 48

N49 12

N50 30

N51 34

N51 37

N49 45

$\begin{array}{ll}W 111 & 41 \\ W 119 & 53\end{array}$

6B37A6A3

953

6A $1 B 6 A 4$

10 40

$w 11045$

W115 11

$\begin{array}{ll}W 112 & 33\end{array}$

$W 118$
$W$

$W 118 \quad 39$

$\begin{array}{lll} & 20\end{array}$

$W 11933$

W133 24

$W 12031$

W125 59

W129 24

W124 41

W117 58

W117 54

W 12932

$W 12925$
$W 13745$

$W 13745$

$\begin{array}{ll}W 126 & 30 \\ W 120 & 18\end{array}$

$W 12018$
$W 129$
$W$

W129 50

W131 55

W125 31

$W 12326$

W126 26

W120 36*

W126 52

W129 15

W133 29

W127 25*

W130 25

W126 52

W126 52

$\begin{array}{ll}W 121 & 22 \\ W 116 & 58\end{array}$

$w 11658$

$\begin{array}{ll}W 119 & 03 \\ W 127 & 14\end{array}$

$W 127$
$W 127$

W127 25

W118 04

$W 12100$
$W 11954$

$W 11954$

$W 124$
$W$

6B37A6A3

6B

6А 1C6A3

GA37A6A3

6A 1B6A4

2A37A2A6

6B37A6A3

7D5C 10A3+

3B4B5A5

2D456B5A4

3E 46C7A 4

3B45B5A

7C1C $10 A 3$

$7 C 1 C 10 A 2+$
$401 C 7 A 4+$

4D45B7A5+

3B 45B5A6

3A5C11A3

3A5C $10 A$

3B 45C5AG

3A5B11A4+

2045C3A

2A 45C3A4

4B4C7A

3A5C5A

$3 A 45 C 5 C$

3C45C5A

7D14C1OA2+

$3 B 4 C 5 B$

3 C 45C5A

3C 4C5A

3B 4C5A

603C6A8

4C4C7A

4D4B7A5

2D456B3A4

3C45A5A6

4C 4C7A

3 C4A5A4
$9 A 5 B 5 A 3+$

4A IC7A

$W 11651$
$W$

$3 A 45 C 3 A 4$ 
BRALORNE AND PIONEER MINES

3B 4B5A5 BRENDA MINE BRISCO

BRI TANNIA MINE

CU MO

BROMLEY GLACIER AREA

BUCCANEER AND MUSKETEER MINES

CU ZN AU AG FES

$W 12000$

$W 11619$

$3045 C 7 A$

BUGABOO CREEK

$\mathrm{MO} \mathrm{CU}$

N49 36

N55 57

W12309

AU

BUGABOO CREEK (CONQUEROR ETC)

BULL RIVER

BULL RIVER MINE

BURNABY ISLAND (JIB, ETC.)

BYRNNOR MINE

\section{CAMBORNE AREA}

CAMBRIAN CHIEFTAIN MINE

CARIBOO DISTRICT

CARIBOO HEART RANGE (NORTHSTAR)

CARIBOO, GOLD QUARTZ AND ISLAND MOUNTAIN MINES

CARIBOO-AMELIA MINE

CARIBOO-HUDSON MINE

CARMI

CARNES CREEK (MASTODON)

CASSIAR (MOUNT MCDAME)

CATFACE RANGE (CATFACE)

CHAPELLE

CHAPUT MINE

CHEHAL IS RIVER (SENECA)

FE

GYP

45049

N50 49

N49 43

FE

ZN PB

$W 12544$

W116 39

$W 12430$

W115 10

$\begin{array}{lll}W 11523 & 23\end{array}$

FE

AU AG PB ZN

AU

CU

$A U$
$A U$
$A U W$

MO

ZN PB

ASB

CU MO

$A U$ AG

ZN CU AG AU

CHERRY CREEK

CHU CHUA (CC)

CHUTANL I LAKE

CHUYAZEGA CRE

CLARK RANGE
CLEAR CREEK (GEM, ETC.)
CLEARWATER PEAK AREA

COLDWATER RIVER (HOPE, MAG)

COLUMBIA TUNGSTEN MINE

COMSTOCK MOUNTAIN (YREKA)

COPPER CREEK AREA

COPPER MOUNTAIN AND INGERBELLE MINES

COQUIHALLA-SPIDER PEAK GOLD BELT (CAROLIN)

CRAIGMONT MINE

DAVENPORT CREEK

DEASE LAKE ARE

DIVIDEND MINE

DRIFTPILE CREEK

DRIFTWOOD RIVER (DRIFTWOOD)

DRUM LUMMON

DUCKLING CREEK AREA (LORRAINE ETC.)

AU

CU $Z N$ AU $A G$

CU MO

MG

$\mathrm{CU}$

MO

AG CU PB ZN

CU

HG

HG

AU

CU FE

MO

AU CU

PB ZN BA

$\mathrm{CU}$

CU AU AG

DULLIS (SOUAW) CREEK

CU

DUNCAN

ECSTALL RIVER AREA

EMERALD GLACIER MINE

EMERALD-INVINCIBLE MINE

ENDAKO MINE

ERIKSON CREEK (VOLLAUG, CORNUCOPIA)

PB ZN

FES ZN CU

$P B$ ZN AG

$W$

MU

AU
N52 24

N49 O3

N50 46

N49 40

N52 56

N56 03

N53 05

$\begin{array}{ll}N 49 & 07\end{array}$

N52 58

N49 31

N51 15

N59 19

N49 15

N57 17

N50 16

N50 16

N49 19

N51 23

N53 2

N54 58

N49 10

N49 43

N51 47

N49 43

N53 10

N50 27

N50 49

N49 20

N49 31

N50 12

N59 52

N58 44

N49 01

N58 04

N55 41

N53 57

N55 54

N60 00

N50 22

N53 52

N53 44

N49 06

N54 02

N59 29

N59 15
$\begin{array}{lll}W 131 & 18\end{array}$

$\begin{array}{ll}W 125 & 27\end{array}$

$W 11737$
$W 123$

W12355

W121 28

$W 126 \quad 15$

W121 33

$\begin{array}{lll}W 119 & 17\end{array}$

$W 12121$

W119 10

W118 07

W129 49

W125 59

$W 12707$

W118 56

W12157

W118 35

W120 O3

W124 30

W122 23

W114 25

$W 12144$

W120 22

W121 01

W121 35

W127 34

$\begin{array}{lll}W & 120 & 47\end{array}$

W120 32*

W121 18*

$W 12055$

W133 O5

$\begin{array}{lll}W 130 & 09\end{array}$

W11930

W125 55

W126 36

W12900

W125 19

W137 07

W116 57

$\begin{array}{ll}W 129 & 30 \\ W 127 & 15\end{array}$

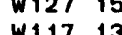

W117 13

W125 06

W134 14

W129 40

3D5B711A5

3C45C5A6

4A4C7A4-

2A45C3A4

$6 A 1-6 C 3$

3E TC7A

2A45B3A

2A456B3A4

4D5C7A4+

3A5C 3A

4A3B 4B7+

4A TB7A3+

4A45C7AG

4A 1C7A

7 C 1C7A4t

5E58A5A-

3C 45B5A6

4C 45C7A 4

$4015 C 7 A$

305C 11 A 4

पA3C $4 A B$

3D5B 11 A3

3C45C5A

$3 A 1 C 10 A 1+$

3B 4B5A 7

3B 4C5A

404C7A

1A 1C7A

$843 C 7 A 6+$

$3 A 25 B 5 B 4$

$4 A 58 C 7 B$

2D45B3A 4

$3 B 15 C 5 A$

4A3C4A7+

4B5C $3 A$

7C IC $10 A 3$

3E 4C 1A

4A3C4AB

7C1B10A2+

$3 D 15 B 11 \mathrm{~A}$

C7A5+

1A 14B3A5

3B4A5A5

4A5C7A3+ 


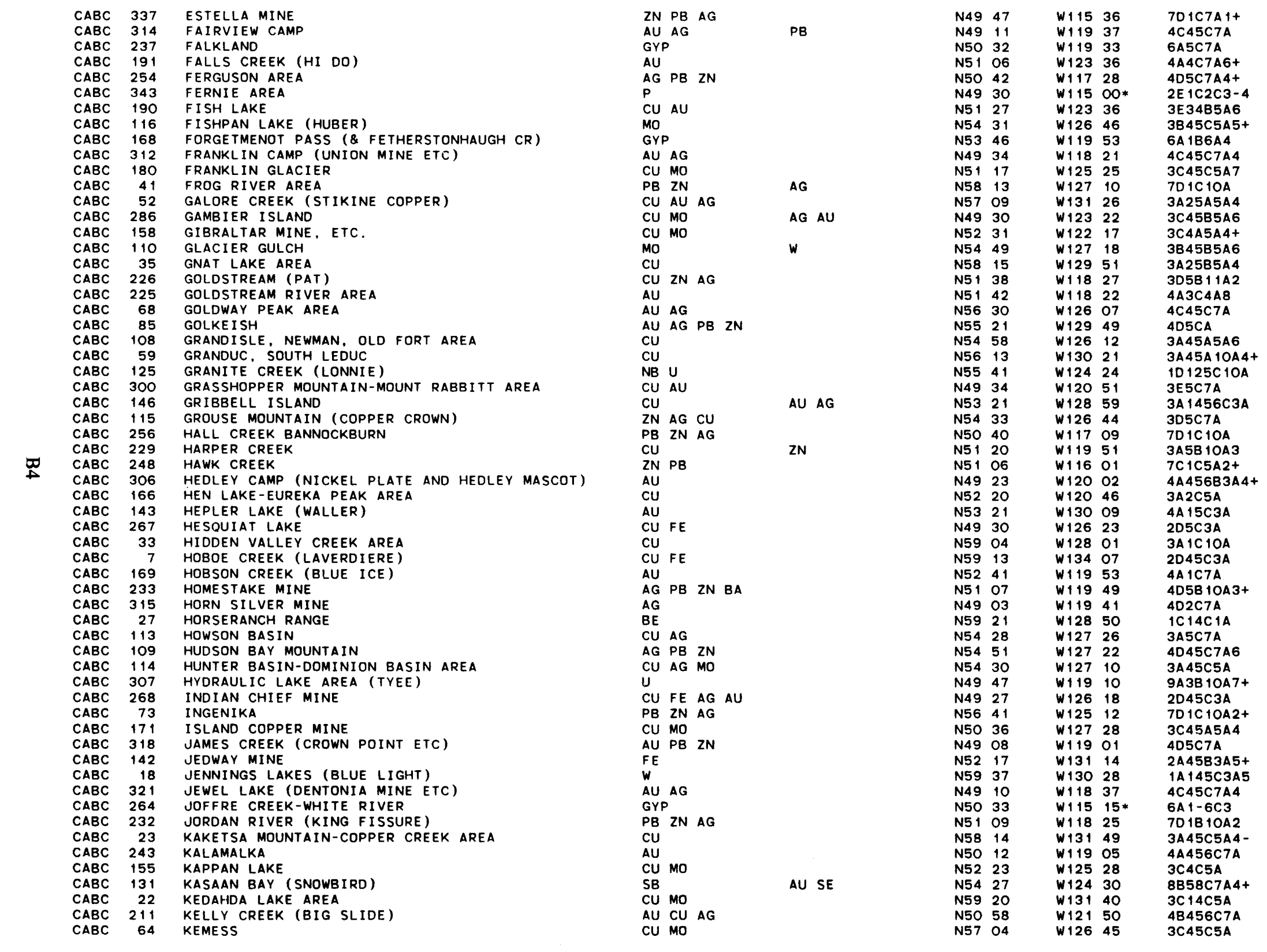




\begin{tabular}{|c|c|c|}
\hline $\begin{array}{l}\text { CABC } \\
\text { CABC }\end{array}$ & $\begin{array}{l}224 \\
235\end{array}$ & $\begin{array}{l}\text { KINBASKET LAKE } \\
\text { KINGF I SHER CREEK }\end{array}$ \\
\hline CABC & 92 & KLEANZA MOUNTAIN (COLUMARIO ETC.) \\
\hline CABC & 326 & KOKANEE CREEK (MOLLY GIBSON) \\
\hline CABC & 265 & KOOTENAY RIVER \\
\hline CABC & 38 & KUTCHO CREEK (JEFF) \\
\hline CABC & 37 & KUTCHO CREEK (LETAIN) \\
\hline CABC & 128 & KWANIKA CREEK (HOGAN, ETC.) \\
\hline CABC & 308 & LASSIE LAKE AREA (FUKI, DONEN, BLIZZARD) \\
\hline CABC & 39 & LEGUIL CREEK (BEAR, MOOSE) \\
\hline CABC & 170 & LEMPRIERE (VERITY) \\
\hline CABC & 306A & LIGHTNING PEAK (WATERLOO) \\
\hline CABC & 48 & LIMPOKE CREEK (LIMPOKE) \\
\hline CABC & 451 & LINDOUIST LAKE (DEER HORN) \\
\hline CABC & 301 & LODESTONE MOUNTAIN AREA \\
\hline CABC & 218 & LORNEX, HIGHMONT (HIGHLAND VALLEY) \\
\hline CABC & 139 & LOUISE ISLAND (IRON DUKE) \\
\hline CABC & 40 & LOWER LIARD CROSSING (GEM, ETC.) \\
\hline CABC & 266 & LUSSIER RIVER \\
\hline CABC & 272 & LYNX MINE \\
\hline CABC & 212 & MAGGIE \\
\hline CABC & 124 & MANSON CREEK AREA \\
\hline CABC & 28 & MARBLE CREEK (S.Q.E.) \\
\hline CABC & 173 & MARBLE RIVER \\
\hline CABC & 340 & MARYSVILLE \\
\hline CABC & 341 & MAYOOK \\
\hline CABC & 305 & MCBRIDE CREEK (ASH, NOLA) \\
\hline CABC & 156 & MCCL INCHY LAKE \\
\hline CABC & 30 & MCDAME CREEK \\
\hline CABC & 58 & MCQUILLAN RIDGE (MAX) \\
\hline CABC & 187 & MENZIES BAY \\
\hline CABC & 260 & MINERAL KING MINE \\
\hline CABC & 197 & MINTO AREA \\
\hline CABC & 57 & MITCHELL CREEK-SULPHURETTS CREEK AREA \\
\hline CABC & 262 & MITCHELL RIVER (MAG) \\
\hline CABC & 246 & MONARCH AND KICKING HORSE MINES \\
\hline CABC & 244 & MONASHEE MINE \\
\hline CABC & 200 & MOON CREEK \\
\hline CABC & 105 & MORRISON LAKE AREA \\
\hline CABC & 284 & MOUNT BALDWIN \\
\hline CABC & 231 & MOUNT COPELAND MINE \\
\hline CABC & 107 & MOUNT CRONIN AREA \\
\hline CABC & 181 & MOUNT DARTMOUTH AREA \\
\hline CABC & 25 & MOUNT HASKIN (JOEM) \\
\hline CABC & 294 & MOUNT HENNING \\
\hline CABC & 88 & MOUNT KNAUSS (FIDDLER-DOREEN) \\
\hline CABC & 90 & MOUNT OBRIEN AREA \\
\hline CABC & 183 & MOUNT PERRITT \\
\hline CABC & 12 & MOUNT SANFORD \\
\hline CABC & 102 & MOUNT THOEN AREA \\
\hline CABC & 91 & MOUNT VANARSDOLL \\
\hline CABC & 8 & MOUNT VAUGHAN (ATLIN-RUFFNER) \\
\hline CAEC & 270 & MOUNT WASHINGTON MINE \\
\hline CABC & 157 & MOUSE MOUNTAIN \\
\hline CABC & 78 & MT. BURDEN \\
\hline CABC & 277 & MT. MCOUILLAN AREA (THISTLE) \\
\hline CABC & 15 & MT. OGDEN (NAN) \\
\hline CABC & 43 & MUNCHO LAKE \\
\hline CABC & 120 & NADINA MINE \\
\hline CABC & 119 & NAKINA RIVER AREA (LUCKY SHIP, ETC.) \\
\hline
\end{tabular}

\begin{tabular}{|c|c|c|c|c|c|c|}
\hline PB & $\mathrm{ZN}$ & & & & N5 1 & \\
\hline $2 N$ & PB & $A G$ & $\mathrm{CU}$ & & N50 & 44 \\
\hline AU & & & & & N54 & 33 \\
\hline AG & PB & $\mathrm{ZN}$ & & & N49 & 44 \\
\hline GYP & & & & & N5O & 14 \\
\hline $\mathrm{cu}$ & $Z N$ & $A G$ & & & N58 & 12 \\
\hline ASB & & & & & N58 & 20 \\
\hline CU 1 & мо & & & & N55 & 30 \\
\hline $\mathbf{u}$ & & & & & N49 & 35 \\
\hline BA & & & & & N59 & 45 \\
\hline NB & $u$ & & & & N52 & 22 \\
\hline$A G$ & PB & $\mathrm{ZN}$ & & & N49 & 53 \\
\hline CU & & & & & N57 & 48 \\
\hline AU & AG & $w$ & & & N53 & 22 \\
\hline FE & & & & & N49 & 28 \\
\hline CU & MO & & & & N50 & 26 \\
\hline $\mathrm{FE}$ & & & & & N52 & 59 \\
\hline$F B$ & $3 A$ & & & & N59 & 26 \\
\hline GYP & & & & & N50 & 01 \\
\hline $\mathbf{Z N}$ & $\mathrm{CU}$ & PB & $A G A U$ & CD BA FES & N49 & 34 \\
\hline cu 1 & MO & & & & N50 & 55 \\
\hline$A U$ & & & & & N55 & 45 \\
\hline MO & & & & & N59 & 15 \\
\hline $\mathbf{Z N}$ & AG & & & & N50 & 26 \\
\hline MG & & & & & N49 & 35 \\
\hline GYP & & & & & N49 & 28 \\
\hline CU 1 & мо & & & & N49 & 07 \\
\hline CU & мо & & & & N52 & 01 \\
\hline AU & & & & & N59 & 16 \\
\hline FE & $\mathrm{cu}$ & & & & N56 & 25 \\
\hline CU & & & & $v$ & N50 & 09 \\
\hline ZN & PB & AG & BA & & N5O & 22 \\
\hline AU & $\mathrm{SB}$ & & & & N50 & 54 \\
\hline CU 1 & MO & & & & N56 & 32 \\
\hline MG & & & & & N50 & 47 \\
\hline $\mathrm{ZN}$ & PB & AG & & & N51 & 24 \\
\hline AU & $\mathbf{A G}$ & & & PB ZN & N50 & 08 \\
\hline ASB & & & & & N50 & 45 \\
\hline $\mathrm{cu}$ & & & & & N55 & 12 \\
\hline $\mathrm{Cu}$ & $\mathrm{ZN}$ & $A G$ & & & N49 & 39 \\
\hline мо & & & & & N5 1 & 08 \\
\hline$A G$ & PB & $\mathrm{ZN}$ & & & N54 & 55 \\
\hline cu 1 & мо & & & & N51 & 15 \\
\hline мO & $w$ & & & & N59 & 20 \\
\hline $\mathrm{cu}$ & & & & мо & N49 & 38 \\
\hline AU & $\Delta G$ & PB & $\mathrm{ZN}$ & & N54 & 48 \\
\hline$A U$ & $A G$ & PB & $Z N \mathrm{CU}$ & & N54 & 40 \\
\hline $\mathrm{FE}$ & & & & & N5O & 18 \\
\hline MO & & & & & N59 & 28 \\
\hline$A G$ & PB & $\mathrm{ZN}$ & & & N55 & 23 \\
\hline MO & & & & & N54 & 36 \\
\hline AG & PB & ZN & & & N59 & 44 \\
\hline $\mathrm{Cu}$ & AG & AU & AS & & N49 & \\
\hline $\mathrm{CU}$ & & & & & N53 & 01 \\
\hline $\mathrm{ZN}$ & PB & & & & N56 & \\
\hline $\mathrm{cu}$ & $A \bar{U}$ & AG & & & N49 & 06 \\
\hline MO & & & & & N58 & 27 \\
\hline BA & $F$ & & & & N58 & 55 \\
\hline $\mathrm{ZN}$ & $\mathrm{CU}$ & PB & $\mathbf{A G}$ & & N54 & 05 \\
\hline MO & & & & & N54 & \\
\hline
\end{tabular}

3C4A5A4

W131 42

W126 O5

W115 31*

$W 12535$

$W 12126$

$W 12440$

$W 12950$

$\begin{array}{ll}W 127 & 24 \\ W 115 & 58\end{array}$

$\begin{array}{ll}W 115 & 58 \\ W 115 & 32\end{array}$

$\begin{array}{ll}W 11532 \\ W 120 & 19\end{array}$

$\begin{array}{ll}W 120 & 19 \\ W & 125 \\ W\end{array}$

$\begin{array}{ll}W 125 & 23 \\ W 129 & 22\end{array}$

$\begin{array}{ll}W 129 & 22 \\ W 130 & 32\end{array}$

$\begin{array}{ll}W 130 & 32\end{array}$

$W 11625$
$W 116$

$W 1162$
$W 122$

$W 12246$
$W 130$
$W$

W130 15

$W 11540$

$W 11626$
$W 11828$

W118 28

W12201

$W 12620$

$W 12302$

$W 11829$

$\begin{array}{ll}W & 126 \\ W & 48\end{array}$

$\begin{array}{ll}W 124 & 23 \\ W 129 & 29\end{array}$

$\begin{array}{ll}W 129 & 29 \\ W 120 & 58\end{array}$

$W 12058$

$\begin{array}{ll}W 128 & 27 \\ W 128 & 10\end{array}$

$W$
$W$
$W$

$\begin{array}{ll}W 124 & 54 \\ W 132 & 47\end{array}$

W126 54

2A45C3A5+

8C 1B 1OA2

6A 1B6C2

3D5B $11 A 3$

3C5B5A6

4A3C4AB

3B4B5A5

7B45C7A

601A2A2

6A1C6A3

3C34C5A6

3C 4C5A

4A3C4AB

2D45C3A4t

7D 1B5A1+

8B45C7A6+

3C45C5A4

G01A2A2

7C 1B 1OA2

4C456C7A

3A45B5A6

305C7A

4B 125C

3C45C5A

3C 14B3A6

$3 A 45 C 5 A$

4D3C7A

4D45C7A

3B45C5A 7

4D45C7C

W128 33

$W 13331$

$\begin{array}{lll}W 125 & 18\end{array}$

W122 19

W123 22

W124 37

W13320

$\begin{array}{lll}W & 125 & 43 \\ W & \end{array}$

3B4C5A

4D6C7A

3E 34C7A7

3A5C5A

7C 1C10A2 +

3E 45C3A

3B4C5A

W126 44 4D34B7AG

3B45B5A6 


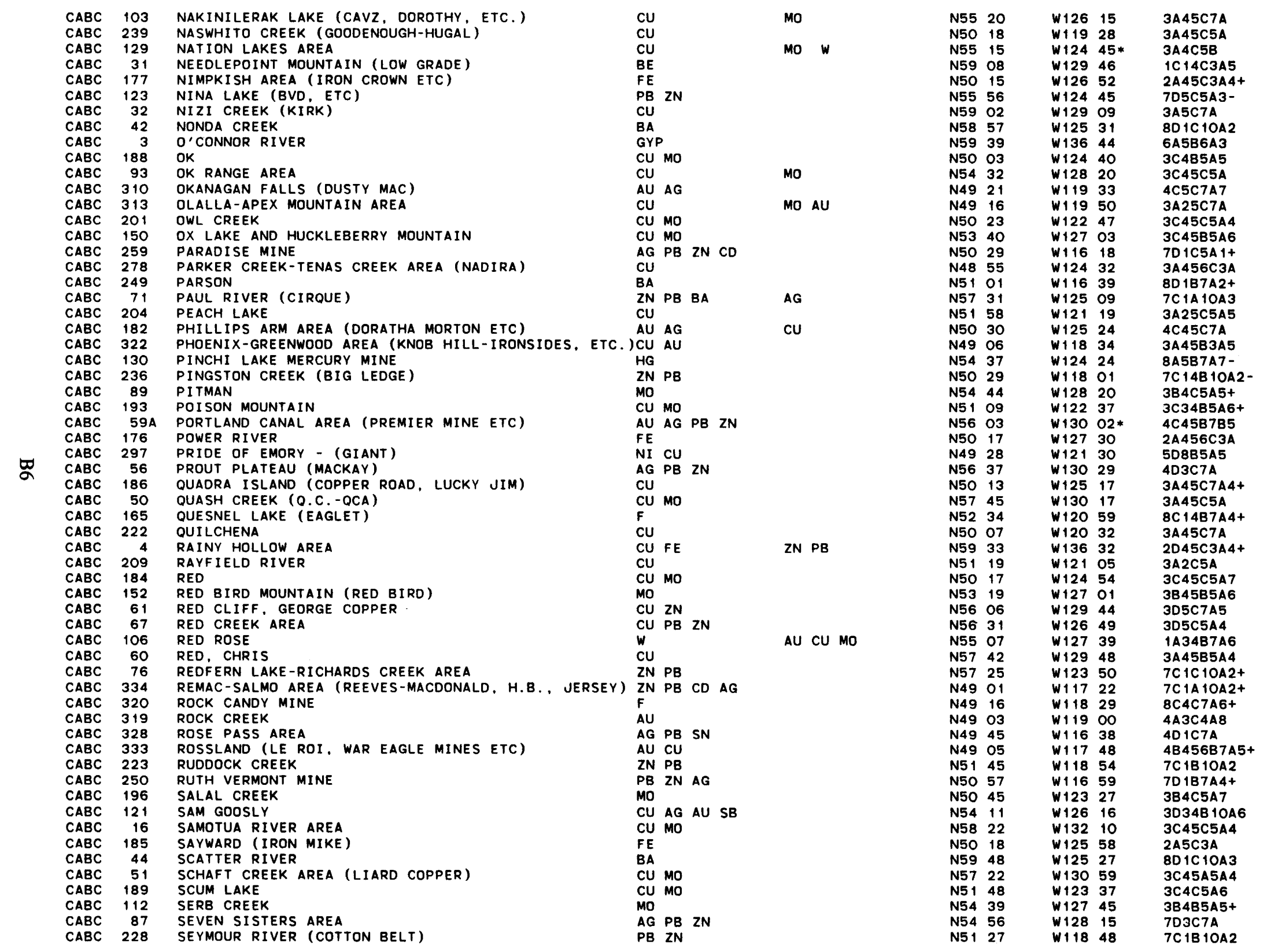


CABC 332 SHEEP CREEK AREA (KOOTENAY BELL, QUEEN, ETC.) $\begin{array}{lll}\text { CABC } & 251 & \text { SILVER GIANT MINE } \\ \text { CABC } & 101 & \text { SILVER STANDARD MINE }\end{array}$

CABC 303 SILVERDAISY MOUNTAIN (CANAM)

CABC 325 SILVERTON-SLOCAN CITY AREA

CABC 296

CABC 136

CABC 324

CABC 19

CABC 55

CABC 288

$\begin{array}{rr}C A B C & 135 \\ C A B C & 69\end{array}$

CABC 344

$\begin{array}{ll}\text { CABC } & 100 \\ \text { CABC } & 175\end{array}$

$\begin{array}{ll}C A B C & 175 \\ C A B C & 220\end{array}$

$\begin{array}{ll}C A B C & 220 \\ C A B C & 329\end{array}$

$\begin{array}{ll}C A B C & 329 \\ C A B C & 295\end{array}$

CABC 28

$\begin{array}{ll}\text { CABC } & 147\end{array}$

$\begin{array}{ll}\text { CABC } & 79 \\ \text { CABC } & 66\end{array}$

CABC 132

CABC 14

CABC 219

$\begin{array}{ll}C A B C & 127 \\ & \end{array}$

CABC 21

$\begin{array}{lll}\forall & \text { CABC } & 138 \\ & \text { CABC } & 199\end{array}$

$\begin{array}{ll}\text { CABC } & 153 \\ & \end{array}$

$\begin{array}{rr}\text { CABC } & 273 \\ \text { CABC } & 99\end{array}$

$\begin{array}{lr}\text { CABC } & 99 \\ \text { CABC } & 330\end{array}$

$\begin{array}{lr}\text { CABC } & 45\end{array}$

$\begin{array}{ll}\text { CABC } & 62 \\ \text { CABC' }^{\prime} & 17\end{array}$

CABC 118

$\begin{array}{rr}\text { CABC } & 94 \\ \text { CABC } & 299\end{array}$

CABC 255

CABC 3014

$\begin{array}{rr}\text { CABC } & 13 \\ & \end{array}$

CABC
CA4

CABC 287

$\begin{array}{rr}C A B C & 54 \\ C A B C & 194\end{array}$

$\begin{array}{ll}\text { CABC } & 194 \\ & 137\end{array}$

CABC 192

CABC 271

CABC 210

CABC 75

CABC 95

CABC 238
CABC

CABC 36

$\begin{array}{ll}\text { CABC } & \mathbf{2 4 2} \\ \text { CABC } & 245\end{array}$

CABC $\quad 339$

SIWASH CREEK AREA

SKIDEGATE (SOUTHEASTER)

SLOCAN-AINSWORTH AREA

SLOUGH MOUNTAIN

SNIPPAKER CREEK (E. \& L.)

SOOKE PENINSULA

SPECOGNA

SQUINGULA RIVER AREA

ST. EUGENE MINE

STERRITT CREEK AREA (LAURA, ETC)

STOREY CREEK

STUMP LAKE AREA (NICOLA)

SULLIVAN MINE

SUMMERS CREEK (AXE)

SUNRO MINE

SURF INLET MINE

SURF POINT MINE

SUSTUT COPPER

STHERLAND RIVER (KID)

SUTLAHINE RIVER AREA (THORN)

SWAKUM MOUNTAIN

TAKLA SILVER (LUSTOUST)

TANZILLA RIVER

TASU MINE

TENQUILLE LAKE AREA

TETACHUCK RIVER (TET, ETC.)

TEXADA MINE

THOML INSON MOUNTAIN

TOAD MOUNTAIN (SILVER KING MINE, ETC.)

TOAD RIVER-RACING RIVER AREA

TOOOOGGONE LAKE (GARNET, SPARTAN)

TOOTSEE LAKE

TREASURE MOUNTAIN (NORTHWEST ETC.)

TREASURE MOUNTAIN (SUMMIT CAMP)

TROUT LAKE

TROUT LAKE

TULSEQUAH AREA

TUNKWA LAKE

TURNAGAIN RIVER (PYRRHOTITE)

TWIN J MINE

TWIN RIVER-VERRETT MOUNTAIN AREA (ISKUT SILVER)

TYAUGHTON LAKE AREA (MANITOU, PHILLIPS)

UNA POINT (EARLY BIRD)

UPPER TASEKO RIVER AREA

VANANDA AREA (MARBLE BAY, CORNELL ETC)

VIDETTE MINE

WASI LAKE AREA (BEVELEY, ETC.)

WEDEENE

WESTWOLD (KENALLEN)

WHEATON CREEK

WHITE ELEPHANT

WHITEROCKS MOUNTAIN

WILDHORSE RIVER
PB ZN AG BA

N49 10 AG ZN PB AU CD

AG PB ZN

MO AG AU

$A G$ CU PB ZN

$A G$ PB ZN CD

CU MO

NI $\mathrm{CU}$

$\mathrm{CU}$

$A U A G$

PB ZN AG

ZN PB CU AG

$\mathrm{CU}$

$A G \quad P B \quad Z N$

PB ZN AG CD SN FES

CU MO

$\mathrm{CU}$ AU

AU CU

AU

MO

CU

HG

AG PB SB ZN

CU MO

FE CU $\mathrm{ZN} \mathrm{CU}$

AG PB

FE CU AU AG

ME

AG CU

$\mathrm{CU}$

AG PB ZN

$A G$ PB $Z N \quad C U$

$C U$

AG PB ZN

MO

ZN PB CU AU AG CD BA

HG

CU NI

$Z N$ CU PB AG AU CD

$A G P B \quad Z N$

HG

CU MO

CU AU AG

CU AU AG

PB ZN AG

FE CU

MO

$A U$
$\mathrm{AU}$
$\mathrm{CU}$

CU MO
W117 07

$W 11629$

W127 37

W121 01

W117 22

W120 20

$W 13200$

W117 13*

$W 130 \quad 15$

$W 13042$

W123 40

W132 13

$\begin{array}{lll}W 127 & 10\end{array}$

$W \uparrow 1549$

W127 38

W126 55

W120 22

$W 11600$

$W 12032 *$

W124 02

W128 54

W130 35

W126 42

W124 52

W133 48

W120 46

$W 12521$

W125 21

$W 12524$
$W 130$
$W$

W132 02

W122 55

$w 12534$

$W 12434$
$W 127$

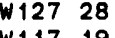

$\begin{array}{lll}W 117 & 19\end{array}$

$w+2525$

$W 12650$

W130 25

$\begin{array}{ll}W 126 & 14\end{array}$

W128 01

$\begin{array}{ll}W 121 & 05 \\ W 117 & 36\end{array}$

$W 11736$
$W 12038 *$

W133 36

W120 49

$W 128$
$W 0$
$W 123$

$W 12347$

$W 13106$
$W 12246$

$W 13211$

$W 12320$

W124 34

W120 54

W125 04

W128 37

$W 11949$

W129O2

W119 33

$W 11945$
$W 11535$
4A 1C7A5+

7D 1C5A2+

4D34C7A6

3A45C5A6

4D4C7A4+

4A C C7A

4D45B7B4+

3C4C5A

5056B5A

DAC7A

AACSA?

7D 1B7A1+

3C4C5A

7D5C3A

4D5C7A4+

7D1A11A1

3C45B5C4

3А36C7A7

$4 B 145 C 7 A$

AA4C7A

4A
3B 4C5 $10 A 4$

3A4C5A

7D5C7A

BA5C7A7 -

4D45C7A

DD $45 B 3 A$

2045B3A4

3C45C5A

3C45C5A

3B34B5A6

3A45C7A4+

3A 16B7A1

3A25C5A4

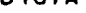

4D3C7A

3A5C5A4t

34C7A

$3 B 45 B 5 A 4+$

4 A3C 4 C8

7D5B 11A4

8 A5C7A

$305 C+1 A 3$

4D25C7A

8A58B7A7

4A5C7A

3С34C5AG

3E 45C3A

4C5C7A

7D 1C 10A2+

उB5C3A

4A3C4A8

4A3C4AB 


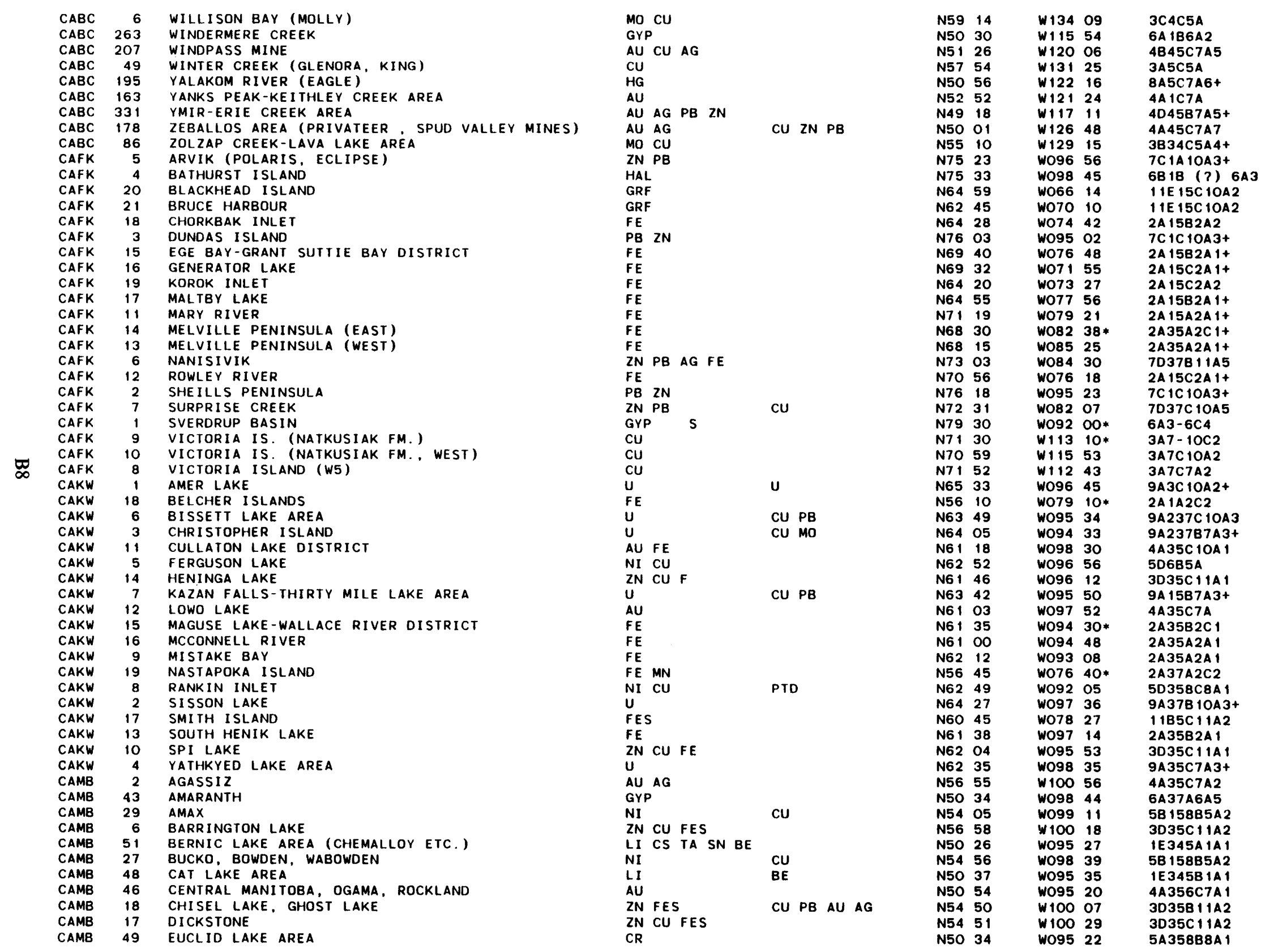




\begin{tabular}{|c|c|c|}
\hline $\begin{array}{l}\text { CAMB } \\
\text { CAMB }\end{array}$ & $\begin{array}{l}53 \\
54\end{array}$ & $\begin{array}{l}\text { FALCON LAKE - STAR LAKE (SUNBEAM ETC.) } \\
\text { FALCON LAKE - WEST BRAINTREE AREA }\end{array}$ \\
\hline CAMB & 14 & FLIN FLON (INCL. SCHIST LAKE, CUPRUS) \\
\hline CAMB & 4 & FOX LAKE MINE \\
\hline CAMB & 33 & FOX RIVER SILL \\
\hline CAMB & 37 & GODS LAKE \\
\hline CAMB & 34 & GODS LAKE (BIL) \\
\hline CAMB & 41 & GOLD ISLAND \\
\hline CAMB & 47 & GUNNAR \\
\hline CAMB & 16 & GURNEY \\
\hline CAMB & 42 & GYPSUMVILLE \\
\hline CAMB & 40 & HIGH ROCK ISLAND \\
\hline CAMB & 32 & HYERS ISLAND \\
\hline CAMB & 36 & JOWSEY I SLAND \\
\hline CAMB & 9 & JUNGLE, BOB \\
\hline CAMB & 1 & KASMERE LAKE AREA \\
\hline CAMB & 23 & LAGUNA \\
\hline CAMB & 5 & LASTHOFE LAKE \\
\hline CAMB & 39 & LINKLATER ISLAND \\
\hline CAMB & 3 & LYNN LAKE \\
\hline CAMB & 7 & MACBRIDE LAKE \\
\hline CAMB & 28 & MANIBR IDGE \\
\hline CAMB & 30 & MOAK, MYSTERY \\
\hline CAMB & 44 & NEEPAWA \\
\hline CAMB & 35 & NO NAME \\
\hline CAMB & 15 & NORTH STAR, DON JON \\
\hline CAMB & 50 & OISEAU (BIRD) RIVER AREA \\
\hline CAMB & 21 & OSBORNE LAKE \\
\hline CAMB & 25 & PIPE \\
\hline CAMB & 38 & PORCUPINE MOUNTAIN (BIRCH RIVER) \\
\hline CAMB & 19 & REED LAKE \\
\hline CAMB & 8 & RUTTAN LAKE \\
\hline CAMB & 45 & SAN ANTONIO \\
\hline CAMB & 10 & SHERIDON (SHERRITT GORDON) \\
\hline CAMB & 52 & SILVER PLAINS \\
\hline CAMB & 13 & SNOW LAKE (NOR-ACME) \\
\hline CAMB & 26 & SOAB, GRASS RIVER \\
\hline CAMB & 20 & SPRUCE POINT \\
\hline CAMB & 22 & STALL LAKE, ANDERSON LAKE \\
\hline CAMB & 31 & THOMPSON, BIRCHTREE \\
\hline CAMB & 12 & VAMP (MARCON) \\
\hline CAMB & 24 & WEKUSKO LAKE AREA \\
\hline CAMB & 11 & WIM \\
\hline CAMK & 43 & ACK GROUP \\
\hline CAMK & 42 & AGRICOLA LAKE (YAVA) \\
\hline CAMK & 34 & ARSENO LAKE (DINGO) \\
\hline CAMK & 45 & BACK RIVER \\
\hline CAMK & 18 & BARRY ISLANDS \\
\hline CAMK & 74 & BIG HILL LAKE \\
\hline CAMK & 63 & BIG SPRUCE LAKE \\
\hline CAMK & 84 & BLACHFORD LAKE COMPLEX \\
\hline CAMK & 68 & BLAISDELL LAKE (1) \\
\hline CAMK & 68A & BLAISDELL LAKE (2) \\
\hline CAMK & 100 & BLANCHET ISLAND \\
\hline CAMK & 82 & BUCKHAM LAKE AREA \\
\hline CAMK & 67 & CAMLAREN MINE AREA \\
\hline CAMK & 27 & CAMSELL RIVER SILVER (SILVER BAY) \\
\hline CAMK & 55 & CAP MOUNTAIN \\
\hline CAMK & 87 & CAVALIER \\
\hline CAMK & 66 & CLAN LAKE \\
\hline
\end{tabular}

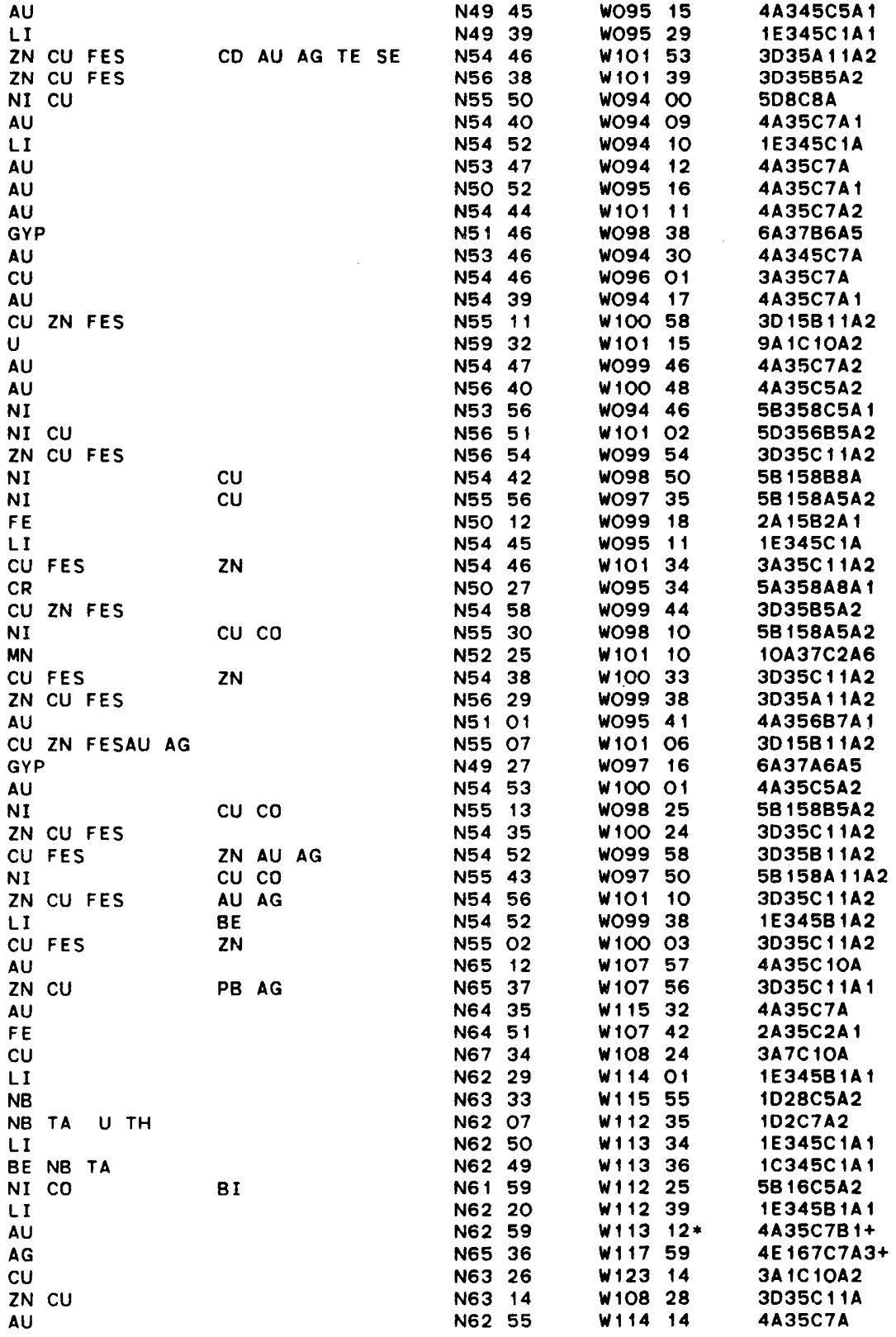




\begin{tabular}{|c|c|c|}
\hline CAMK & 54 & COATES LAKE (REDSTONE, KVALE) \\
\hline CAMK & 73 & CON-RYCON AND NEGUS MINES \\
\hline CAMK & 26 & CONTACT LAKE \\
\hline CAMK & 40 & CONTWOYTO LAKE AREA \\
\hline CAMK & 39 & CONTWOYTO LAKE DISTRICT \\
\hline CAMK & 89 & COPPER PASS MINES (GOGO) \\
\hline CAMK & 11 & COPPERMINE DISTRICT \\
\hline CAMK & 65 & DISCOVERY MINE AREA \\
\hline CAMK & 3 & DOT GROUP (NO. 47) \\
\hline CAMK & 102 & EASTER ISLAND \\
\hline CAMK & 8 & EL BONANZA \\
\hline CAMK & 7 & ELDORADO DISTRICT (INCL \\
\hline CAMK & 32 & EXMOUTH LAKE \\
\hline CAMK & 5 & FAULT RIVER \\
\hline CAMK & 95 & FLAT RIVER (CANADA TUNGSTEN) \\
\hline CAMK & 80 & FRANCOIS LAKE AREA \\
\hline CAMK & 15 & GALENA POINT \\
\hline CAMK & 21 & GAYNA RIVER \\
\hline CAMK & 46 & GODLIN LAKES AREA \\
\hline CAMK & 38 & HACKETT RIVER \\
\hline CAMK & 52 & HAYHOOK \\
\hline CAMK & 83 & HEARNE CHANNEL-DREVER \\
\hline CAMK & 19 & HIGH LAKE \\
\hline CAMK & 69 & HOMER LAKE \\
\hline CAMK & 1 & HOPE BAY (1) \\
\hline CAMK & 2 & HOPE BAY (2) \\
\hline CAMK & 104 & HORNBY CHANNEL AREA \\
\hline CAMK & 29 & HOTTAH LAKE AREA \\
\hline CAMK & 48 & HOWARDS PASS \\
\hline CAMK & 6 & HUNTER BAY (SLOAN RIVER) \\
\hline CAMK & 86 & INDIAN MOUNTAIN LAKE (BB) \\
\hline CAMK & 36 & INDIN LAKE \\
\hline CAMK & 31 & IZOK LAKE \\
\hline CAMK & 10 & JUNE \\
\hline CAMK & 51 & KEELE RIVER (JUNE CREEK) \\
\hline CAMK & 91 & LAC DUHAMEL (GO GO NO. 3) \\
\hline CAMK & 49 & LENED \\
\hline CAMK & 98 & LIARD RIVER \\
\hline CAMK & 59 & LOU LAKE \\
\hline CAMK & 96 & LUCKY LAKE \\
\hline CAMK & 25 & MACKENZIE BASIN \\
\hline CAMK & 62 & MACKENZIE TROUGH \\
\hline CAMK & 47 & MACMILLAN PASS (MACTUNG) \\
\hline CAMK & 90 & MARY GROUP \\
\hline CAMK & 58 & MAZENOD LAKE \& PRECAMBRIAN INLIER AREA \\
\hline CAMK & 109 & MC INNIS LAKE \\
\hline CAMK & 94 & MERIDIAN LAKE (ANN) \\
\hline CAMK & 64 & MOSHER LAKE (BR) \\
\hline CAMK & 4 & MOUNTAIN LAKE \\
\hline CAMK & 92 & MURKY CHANNEL (SPD) \\
\hline CAMK & 12 & MUSKOX INTRUSION (i) \\
\hline CAMK & 13 & MUSKOX INTRUSION (2) \\
\hline CAMK & 99 & NAHANNI BUTTE \\
\hline CAMK & 35 & NORR IS LAKE \\
\hline CAMK & 108 & O'CONNOR LAKE AREA \\
\hline CAMK & 57 & OLD FORT ISLAND \\
\hline CAMK & 101 & OUTPOST I SLAND \\
\hline CAMK & 78 & PAYNE LAKE AREA (PAT) \\
\hline CAMK & 53 & PER \\
\hline CAMK & 105 & PINE POINT AREA \\
\hline
\end{tabular}

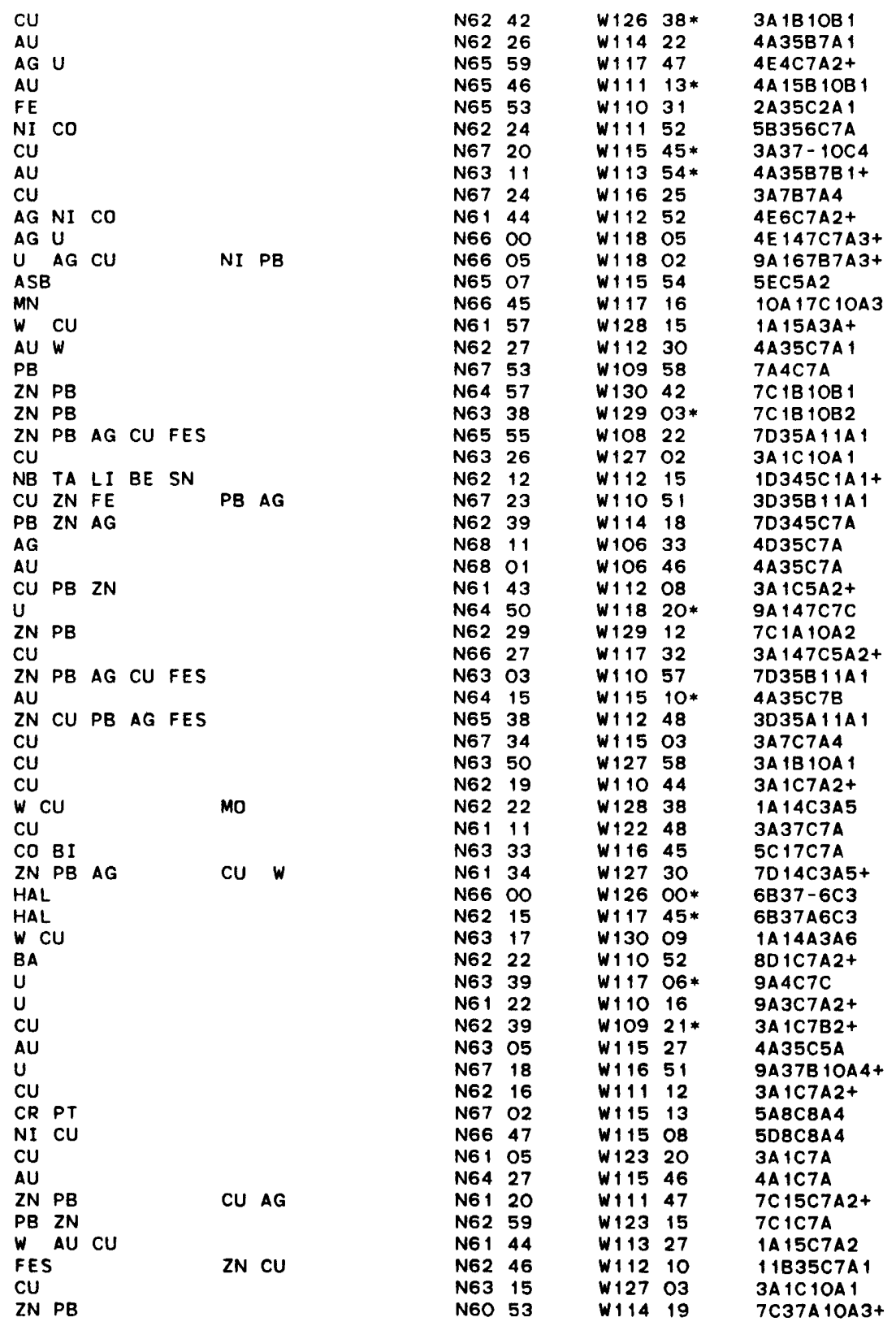




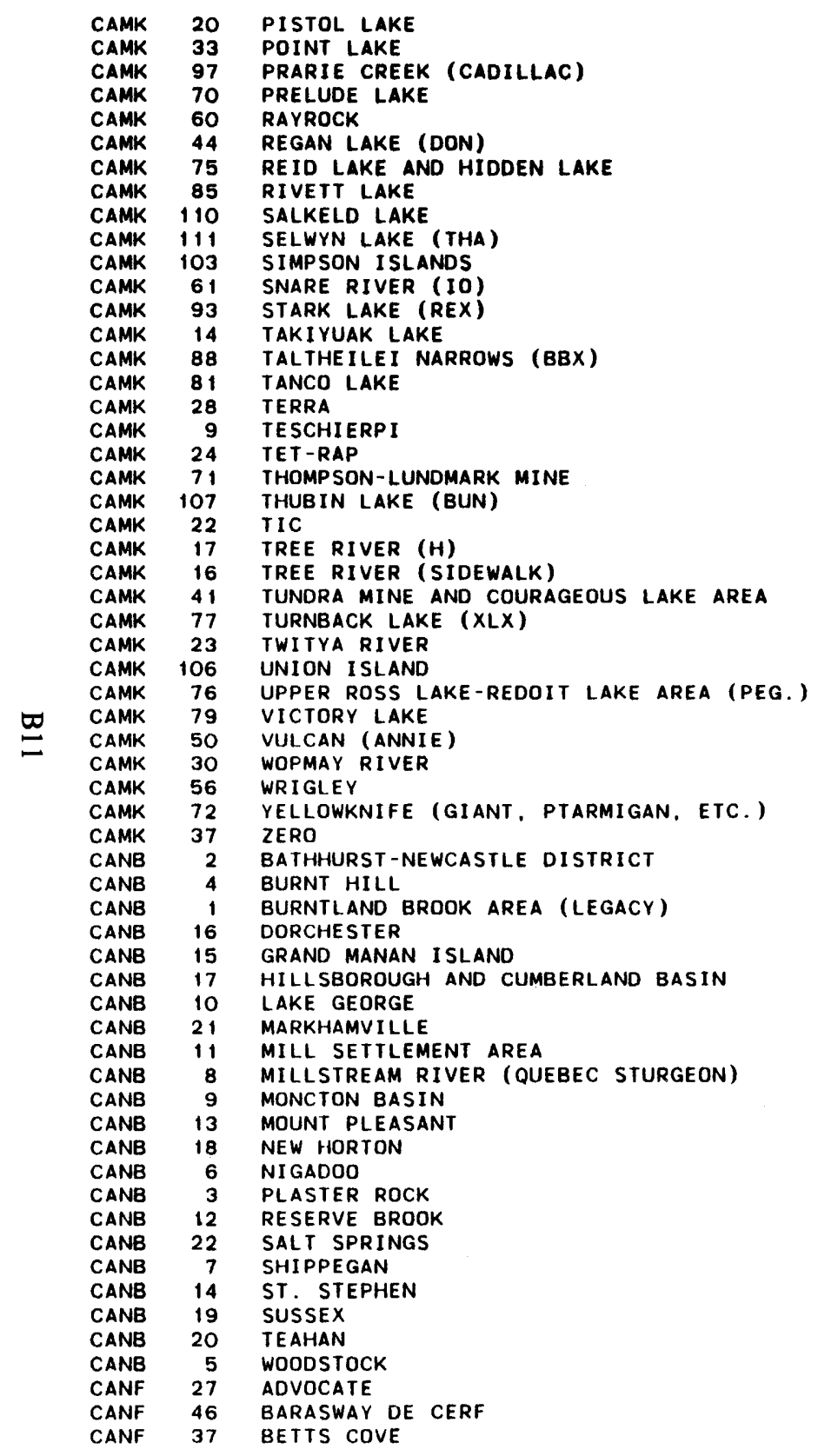

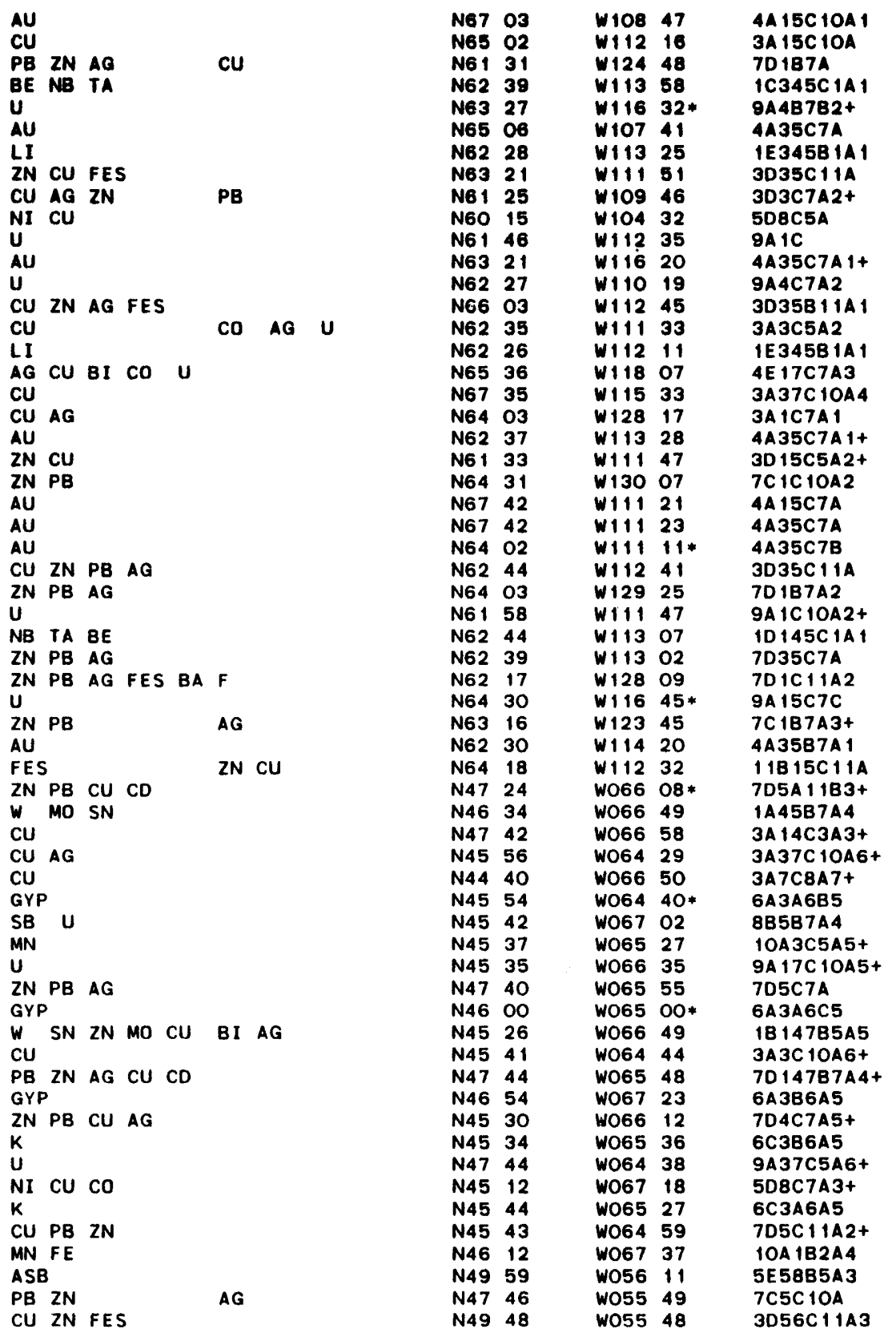




\begin{tabular}{|c|c|c|}
\hline CANF & 21 & BLUFF HEAD \\
\hline CANF & 32 & BUCHANS \\
\hline CANF & 41 & BURNT HILL \\
\hline CANF & 13 & CAROL LAKE \\
\hline CANF & 42 & CODROY RIVER DISTRICT \\
\hline CANF & 50 & COLLIER COVE \\
\hline CANF & 52 & CONCEPTION BAY (BRIGUS) \\
\hline CANF & 17 & DANIEL'S HARBOUR \\
\hline CANF & 26 & FISCHELLS \\
\hline CANF & 24 & FLAT BAY \\
\hline CANF & 18 & FLEUR DE LYS (HODDER) \\
\hline CANF & 16 & GOOSE COVE \\
\hline CANF & 35 & GREAT BURNT LAKE \\
\hline CANF & 19 & GREGORY RIVER \\
\hline CANF & 45 & GREY RIVER \\
\hline CANF & 31 & GULL POND (GULLBRIDGE) \\
\hline CANF & 23 & INDIAN HEAD \\
\hline CANF & 44 & ISLE AUX MORTS RIVER (RIOCANEX) \\
\hline CANF & 4 & KIGLAPAIT INTRUSION \\
\hline CANF & 11 & KITTS \\
\hline CANF & 5 & KNOB LAKE DISTRICT \\
\hline CANF & 49 & LA MANCHE \\
\hline CANF & 38 & LITTLE BAY AND WHALESBACK \\
\hline CANF & 10 & MAKKOVIK \\
\hline CANF & 53 & MANUELS \\
\hline CANF & 12 & MICHELIN \\
\hline CANF & 8 & MORAN LAKE AREA \\
\hline CANF & 40 & MORETON'S HARBOUR \\
\hline CANF & 39 & PILLEY'S ISLAND \\
\hline CANF & $40 A$ & POINT LEAMINGTON \\
\hline CANF & 22 & PORT AU PORT PEN. (BOSWARLOS) \\
\hline CANF & 29 & RAMBLER \\
\hline CANF & 47 & RECONTRE EAST \\
\hline CANF & 1 & ROSWELL HARBOUR \\
\hline CANF & 2 & SAGELEK BAY \\
\hline CANF & 3 & SAGELEK BAY-HEBRON DISTRICT \\
\hline CANF & 48 & SAINT LAWRENCE \\
\hline CANF & 6 & SAWYER LAKE \\
\hline CANF & 9 & SEAL LAKE DISTRICT \\
\hline CANF & 54 & SILVER CLIFF \\
\hline CANF & 30 & SOPS ARM (BROWNING) \\
\hline CANF & 25 & STEEL MOUNTAIN \\
\hline CANF & 43 & STRICKLAND-LA POILE \\
\hline CANF & 7 & TEN MILE LAKE \\
\hline CANF & 28 & IERRA NOVA \\
\hline CANF & 36 & TILT COVE \\
\hline CANF & 34 & TULK'S POND \\
\hline CANF & 33 & VICTORIA \\
\hline CANF & 51 & WABANA \\
\hline CANF & 14 & WABUSH \\
\hline CANF & 15 & WILSON LAKE \\
\hline CANF & 20 & YORK HARBOUR \\
\hline CANS & 15 & ANT I GONISH \\
\hline CANS & 9 & BRAS D'OR LAKE DISTRICT \\
\hline CANS & 44 & BRAZIL LAKE \\
\hline CANS & 26 & BROOKFIELD \\
\hline CANS & 29 & CARIBOU DISTRICT \\
\hline CANS & 3 & CHE T I CAMP \\
\hline CANS & 7 & COXHEATH \\
\hline CANS & 2 & DINGWALL \\
\hline
\end{tabular}

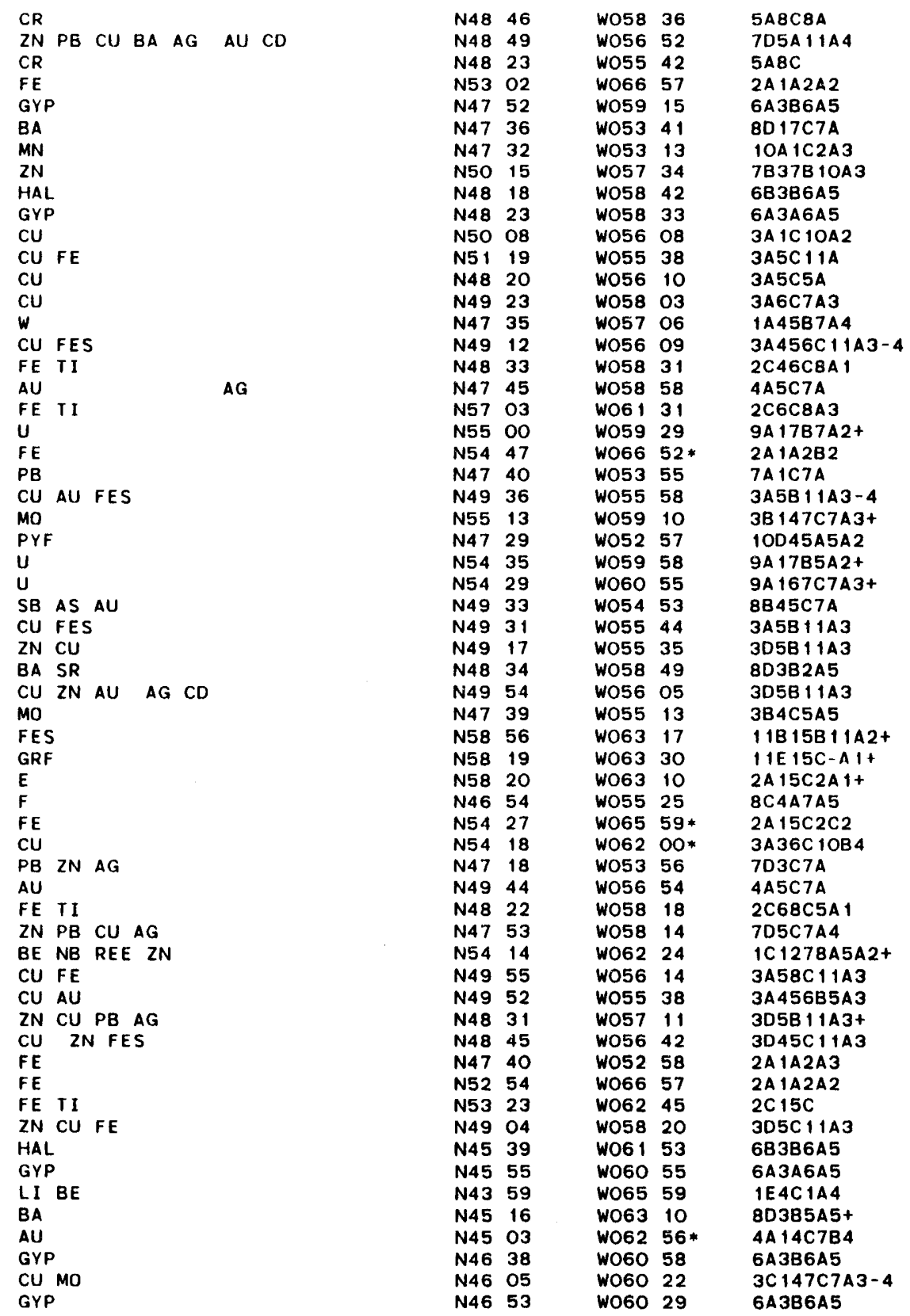




\begin{tabular}{|c|c|c|}
\hline CANS & 43 & EAST KEMPTVILLE \\
\hline CANS & 23 & EAST MOUNTAIN \\
\hline CANS & 10 & GABARUS \\
\hline CANS & 30 & GAYS RIVER \\
\hline CANS & 33 & GOLDENVILLE \\
\hline CANS & 32 & HALIFAX-HANTS DISTRICT \\
\hline CANS & 24 & HILDEN \\
\hline CANS & 5 & LAKE AINSLIE \\
\hline CANS & 12 & LAKE ENON \\
\hline CANS & 42 & LEIPSIGATE \\
\hline CANS & 14 & LIME HILL \\
\hline CANS & 22 & LONDONDERRY \\
\hline CANS & 6 & MABOU \\
\hline CANS & 36 & MARGARETSVILLE DISTRICT \\
\hline CANS & 1 & MEAT COVE \\
\hline CANS & 31 & MILFORD \\
\hline CANS & 25 & MINASVILLE-TENNYCAPE \\
\hline CANS & 41 & MOLEGA AND BROOKFIELD \\
\hline CANS & 34 & NAPPAN \\
\hline CANS & 39 & NEW ROSS \\
\hline CANS & 40 & NEW ROSS DISTRICT \\
\hline CANS & 38 & NICTAUX \\
\hline CANS & 17 & PORT RICHMOND \\
\hline CANS & 19 & PUGWASH \\
\hline CANS & 4 & ROCKY BROOK \\
\hline CANS & 11 & SALMON RIVER (YAVA) \\
\hline CANS & 18 & SEAL HARBOUR AREA \\
\hline CANS & 45 & SHELBURNE DISTRICT \\
\hline lCANS & 27 & SMITHFIELD (LEADVALE) \\
\hline CANS & 13 & STIRLING (MINDAMAR) \\
\hline CANS & 21 & TATAMAGOUCHE AREA \\
\hline CANS & 20 & WALLACE \\
\hline CANS & 35 & WALTON \\
\hline CANS & 28 & WEST GORE \\
\hline CANS & 37 & WINDSOR DISTRICT \& CHEVERIE \\
\hline CAON & 202 & ADMASTON TP. \\
\hline CAON & 173 & AFTON TP. (NEW GOLDEN ROSE) \\
\hline CAON & 181 & AGNEW LAKE DISTRICT \\
\hline CAON & 198 & ALBEMARLE TP. \\
\hline CAON & 128 & ALEXO \\
\hline CAON & 54 & ARGOR ALKALINE COMPLEX \\
\hline CAON & 17 & ARGOSY (NEW JASON) \\
\hline CAON & 136 & ASHLEY \\
\hline CAON & 66 & ATIKWA LAKE (MAYBRUN) \\
\hline CAON & 27 & AVIS LAKE DISTRICT \\
\hline CAON & 33 & BAMAJI LAKE AREA \\
\hline CAON & 213 & BANCROFT AREA (FARADAY ETC ...) \\
\hline CAON & 94 & BEAVER, BADGER, ETC... \\
\hline CAON & 227 & BEDFORD TP. (DESERT LAKE) \\
\hline CAON & 13 & BEE LAKE \\
\hline CAON & 77 & BEILDELMAN BAY AND YOUNG LAKE \\
\hline CAON & 28 & BELANGER TP. - SNAKEWOOD LAKE AREA \\
\hline CAON & 228 & BELMONT TP. (BLAIRTON) \\
\hline CAON & 222 & BELMONT TP. (CORDOVA) \\
\hline CAON & 69 & BENDING LAKE \\
\hline CAON & 6 & BERENS RIVER (GOLSIL) \\
\hline CAON & 10 & BIG BEAVERHOUSE LAKE ALKALINE COMPLEX \\
\hline CAON & 209 & BLACK DONALD \\
\hline CAON & 4 & BORLAND LAKE (BEATRICE) \\
\hline CAON & 139 & BOSTON TP. (ADAMS) \\
\hline
\end{tabular}

\begin{tabular}{|c|c|c|c|c|}
\hline SN & & $C U \quad Z N \quad A G$ & N44 & 06 \\
\hline MN & & & N45 & 25 \\
\hline MO & B I & $\mathrm{CU}$ & N45 & 53 \\
\hline PB & $\mathrm{ZN}$ & & N45 & 01 \\
\hline$A U$ & & & $\mathrm{~N} 45$ & 07 \\
\hline$A U$ & & & N44 & 50 \\
\hline $\mathbf{s}$ & & & N45 & 19 \\
\hline $\mathbf{F}$ & BA & & N46 & 07 \\
\hline SR & & & N45 & 48 \\
\hline$A U$ & & & N44 & 19 \\
\hline $\mathbf{Z N}$ & & PB & N45 & 46 \\
\hline FE & & & N45 & 28 \\
\hline HAL & & & N46 & 02 \\
\hline $\mathrm{CU}$ & & & $\mathrm{N} 45$ & 04 \\
\hline$Z N$ & & & N47 & $\infty$ \\
\hline GYP & & & N45 & $\infty$ \\
\hline MN & & & N45 & 17 \\
\hline$A U$ & & & N44 & 20 \\
\hline HAL & & & N45 & 47 \\
\hline MN & & & N44 & 51 \\
\hline SN & & & N44 & 47 \\
\hline $\mathrm{FE}$ & & & N44 & 55 \\
\hline HAL & & & N45 & 35 \\
\hline HAL & & & N45 & 50 \\
\hline $\mathrm{ZN}$ & $\mathrm{CU}$ & & N46 & 31 \\
\hline PB & $\Delta G$ & & N45 & 52 \\
\hline$A U$ & & & N45 & 12 \\
\hline BE & & & $\mathrm{N} 43$ & 46 \\
\hline PB & $\mathrm{ZN}$ & & N45 & 16 \\
\hline PB & $Z N A G$ & $\mathrm{CU}$ & N45 & 4 \\
\hline cU & & $u$ & N45 & 40 \\
\hline HAL & & $\mathrm{k}$ & N45 & 47 \\
\hline BA & & $A G \quad P B \quad Z N \quad C U$ & N45 & 12 \\
\hline SB & $A U$ & & N45 & 05 \\
\hline GYP & & & N44 & 5 \\
\hline $\mathrm{ZN}$ & & & N45 & 25 \\
\hline$A U$ & & & N46 & 56 \\
\hline$u$ & TH REE & & N46 & 2 \\
\hline $\mathrm{ZN}$ & & PB & N44 & 47 \\
\hline NI & & $\mathrm{cu}$ & N48 & 39 \\
\hline NB & & & N50 & 5 \\
\hline$A U$ & & $A G$ & N5 1 & 2 \\
\hline$A U$ & & $\mathbf{A G}$ & N48 & 01 \\
\hline CU & $A U$ & & $\mathrm{~N} 49$ & \\
\hline $\mathrm{FE}$ & & & N50 & 57 \\
\hline MO & & $\mathrm{CU}$ & N5 1 & \\
\hline $\mathbf{u}$ & TH REE & & N45 & 01 \\
\hline AG & $A U$ & PB ZN & N48 & 19 \\
\hline GRF & & & N44 & 34 \\
\hline AU & $w$ & & N5O & 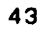 \\
\hline MO & $\mathrm{CU}$ & & N49 & 5 \\
\hline $\mathrm{ZN}$ & $\mathrm{CU} F E$ & & N5O & 58 \\
\hline $\mathrm{FE}$ & & & N44 & \\
\hline$A U$ & & & N44 & 32 \\
\hline $\mathrm{FE}$ & & & N49 & 1 \\
\hline AG & $A U Z N \quad P B$ & & N52 & \\
\hline NB & & & N52 & \\
\hline GRF & & & N45 & \\
\hline AG & PB & & N52 & \\
\hline FE & & & N48 & \\
\hline
\end{tabular}

W065 43 W063 10

W060 06

W063 22

W062 01

W063 40*

W063 19

W061 107

W060 33

W064 37

$\begin{array}{ll}061 & 09\end{array}$

W063 39

W06 127

W065 01

W060 35

W063 25

W063 48

W064 54*

W064 14

W064 27

W064 27

W065 00

W061 16

W061 16

W063 40

W060 53

WO61 38*

WO65 21*

WO63 05

WO60 26

$\begin{array}{ll}\text { WO6 } 15 & 15\end{array}$

$\begin{array}{ll}\text { WO63 } & 15 \\ \text { W063 } & 27\end{array}$

W063 27

$\begin{array}{ll}\text { W064 } & 02 \\ \text { W063 } 47\end{array}$

W063 47

W064 05

W076 42

WO8O 19

WO8 110

W080 49

W080 35

W092 20

W080 54

W080 54

W093 38

W092 25

W077 55

W077 55

W089 38

W076 35

W095 08

W09 110

W092 55

W077 46

W077 47

W092 11

W093 38

WO89 55

W076 55

W094 07

WO79 55
14B3A 4

B 147C5A

7C3B5A5+

4A 1C7A4

4A 14C7B2

8C17B7A5+

8E 3A6A5-6

4A 1C7A

7B 14C3A

2A 17C5A4

6B3B6A5

$3 A 3 C 10 A 7$
$7 B 145 B 3 A 1-4$

6A37A6A5

10A3C5A5

$4 A 14 C 7 B 4$

6B3B6A5

10A4C5A 4

1B4C7A4

6B 1 C2A4

6B3B6A5

$7 D 1 C$

7A37B $10 A 6$

4A 14C7B2

1C4C 1B4

7D17B11A3+

3A37C $10 A 6$

6B3B6A5

8D3A5A5

88 1B7A4+

6A3A6A5

7B5C $10 A 3$

4A35C 10A 1

9E37B 10A2

7B37C $10 A 3$

5B358C5A 1

1028A5A2

4A35C7A1

3A35C10A

उA35A

2A $35 A 2 A 1$

3B4C5A

4D367C7B4+

$11 E$ 1B 1OA4 -

4A35C7A

3C4C5A

3D 35C 1 1A 1

2A 156C3A4

4A6C7A4+

2A35A2A1

4C35C7A 1

1028C5A4

4D 35C $10 A$

2A35B2A 1 


\begin{tabular}{|c|c|c|}
\hline CAON & 210 & BROUGHAM TP. \\
\hline CAON & 163 & BRUCE MINES \\
\hline CAON & 98 & BURROWS LAKE \\
\hline CAON & 172 & BURWASH LAKE \\
\hline CAON & 211 & CALABOGIE DISTRICT \\
\hline CAON & 236 & CALEDONIA-HAGERSVILLE-CAYUGA DISTRICT \\
\hline CAON & 1 & CARB LAKE ALKALINE COMPLEX \\
\hline CAON & 199 & CARDIFF AND MONMOUTH TPS. \\
\hline CAON & 116 & CARGILL ALKALINE COMPLEX \\
\hline CAON & 72 & CARPENTER TP. \\
\hline CAON & 189 & CASEY + HARRIS TPS. \\
\hline CAON & 31 & CENTRAL PATRICIA \\
\hline CAON & 115 & CLAY-HOWELLS ALKALINE COMPLEX \\
\hline CAON & 190 & COBALT DISTRICT (COLEMAN + BUCKE TPS.) \\
\hline CAON & 19 & COCHENOUR WILLIAMS, CAMPBELL, ETC. \\
\hline CAON & 107 & COLDWELL COMPLEX \\
\hline CAON & 175 & CRAIG TP. (STRALAK) \\
\hline CAON & 194 & CROCAN AND TIMBER LAKES \\
\hline CAON & 155 & CUNNINGHAM TP. (ALDRA) \\
\hline CAON & 180 & DAVIS TP. (NORSTAR LAKE) \\
\hline CAON & 154 & DESROSIERS TP. (ALIKE LAKE) \\
\hline CAON & 55 & DETOUR LAKE \\
\hline CAON & 23 & DIXIE \\
\hline CAON & 71 & DOBIE TP., EMO \\
\hline CAON & 67 & DOG PAW LAKE \\
\hline CAON & 36 & DORAN LAKE \\
\hline CAON & 92 & DORION TP. ( DORION AND OGEMA) \\
\hline CAON & 188 & DRYDEN TP. \\
\hline CAON & 112 & EGO \\
\hline CAON & 159 & ELLIOT LAKE DISTRICT (NORDIC ZONE) \\
\hline CAON & 158 & ELLIOT LAKE DISTRICT (QUIRKE ZONE) \\
\hline CAON & 99 & ELMHIRST TP. \\
\hline CAON & 7 & FAVOURABLE LAKE \\
\hline CAON & 203 & FITZROY TP. (KINGDON) \\
\hline CAON & 138 & GARRISON TP. \\
\hline CAON & 104 & GEORGIA LAKE-JEAN LAKE DISTRICT \\
\hline CAON & 103 & GERALDTON DISTRICT \\
\hline CAON & 102 & GERALDTON IRON RANGE (ERRINGTON TP.) \\
\hline CAON & 234 & GODERICH DISTRICT \\
\hline CAON & 63 & GOLDLUND \\
\hline CAON & 15 & GORDON LAKE - WERNER LAKE \\
\hline CAON & 110 & GOUDREAU \\
\hline CAON & 111 & GOUDREAU DISTRICT \\
\hline CAON & 157 & GOULAIS RIVER \\
\hline CAON & 170 & GOWGANDA \\
\hline CAON & 73 & GRASSY PORTAGE BAY \\
\hline CAON & 22 & GRIFFITH \\
\hline CAON & 208 & GRIFFITH TP. (SPAIN) \\
\hline CAON & 93 & GUNFLINT \\
\hline CAON & 164 & HERMINA AND MASSEY \\
\hline CAON & 56 & HIGH LAKE \\
\hline CAON & 44 & HOLLINGSWORTH LAKE \\
\hline CAON & 122 & HORWOOD LAKE (PENHORWOOD TP.) \\
\hline CAON & 153 & HORWOOD LAKE - COPPELL LAKE AREA- \\
\hline CAON & 87 & HUNTER ISLAND DISTRICT \\
\hline CAON & 224 & HUNT INGTON TP. (HENDERSON-CONLEY) \\
\hline CAON & 81 & HUTCHINSON TP. (ATIKOKAN) \\
\hline CAON & 42 & ISHKI SH LAKE \\
\hline CAON & 24 & JACKSON-MANION \\
\hline CAON & 151 & JARDUN \\
\hline
\end{tabular}

\begin{tabular}{|c|c|c|c|c|c|}
\hline MO & & & & N45 & 19 \\
\hline $\mathrm{CU}$ & & & & N46 & 18 \\
\hline MO & & & & N49 & 55 \\
\hline $\mathrm{FE}$ & & & & N47 & 05 \\
\hline $\mathrm{FE}$ & & & & N45 & 18 \\
\hline GYP & & & & N43 & 05 \\
\hline NB & REE & & & N54 & 48 \\
\hline MO & & $\mathbf{F}$ & $u$ & N45 & 01 \\
\hline$P$ & & NB & REE & N49 & 18 \\
\hline $\mathrm{FE}$ & & & & N48 & 39 \\
\hline$A G$ & $\mathrm{co}$ & NI & $c U$ & N47 & 35 \\
\hline$A U$ & FES & $\mathbf{A G}$ & & N5 1 & 29 \\
\hline$F E$ & & & & N49 & 50 \\
\hline$A G$ & $\mathrm{co}$ & NI & $\mathrm{CU}$ & N47 & 22 \\
\hline AU & & $A G$ & & N51 & 03 \\
\hline $\mathrm{FE}$ & TI CU & & & N48 & 49 \\
\hline $\mathrm{ZN}$ & CU AG FES & & & N46 & 48 \\
\hline KYN & & & & N46 & 33 \\
\hline $\mathrm{ZN}$ & CU FES & & & N47 & 43 \\
\hline AU C & $\mathrm{cu}$ & & & N46 & 39 \\
\hline MO & & & & N47 & 48 \\
\hline$A U$ & & & & N50 & 01 \\
\hline $\mathrm{CU}=$ & $Z N F E$ & & & N5O & 52 \\
\hline $\operatorname{cus} 1$ & NI co & & & N48 & 40 \\
\hline$\Delta U$ & & & & N49 & 20 \\
\hline FE & & & & N5O & 58 \\
\hline PB & & $Z N$ & BA & N48 & 45 \\
\hline KVN & & & & N46 & 30 \\
\hline CU & $A U Z N$ FES & & & N48 & 15 \\
\hline $\mathrm{u}$ & TH REE & & & N46 & 23 \\
\hline U & TH REE & & & N46 & 31 \\
\hline NI & $\mathrm{cu}$ & & & N49 & 49 \\
\hline$u$ & & мо & & N52 & 50 \\
\hline PB & & $\mathrm{ZN}$ & & N45 & 26 \\
\hline$A S B$ & & & & N48 & 32 \\
\hline LI & & & & N49 & 21 \\
\hline$A U$ & & $A G$ & $w$ & N49 & 40 \\
\hline $\mathrm{FE}$ & & & & N49 & 42 \\
\hline HAL & & & & N43 & 44 \\
\hline$A U$ & & & & N49 & 54 \\
\hline NI & $\mathrm{cU}$ & $\mathrm{co}$ & PTD & N5O & 28 \\
\hline FES & & & & N48 & 17 \\
\hline$A U$ & & & & N48 & 19 \\
\hline $\mathrm{FE}$ & & & & N47 & 03 \\
\hline $\mathbf{A G}$ & $\mathrm{CO}$ & NI & $\mathrm{CU}$ & N47 & 40 \\
\hline $\mathrm{Cu}$ & & NI & & N48 & 41 \\
\hline $\mathrm{FE}$ & & & & N5O & 49 \\
\hline MO & & & & N45 & 17 \\
\hline $\mathrm{FE}$ & & & & N48 & 15 \\
\hline $\mathrm{cu}$ & & & & N46 & 15 \\
\hline MO & CU AU & & & N49 & 42 \\
\hline $\mathrm{FE}$ & & & & N5O & 35 \\
\hline$B A$ & & & & N48 & 05 \\
\hline$A U$ & & & & N47 & 55 \\
\hline FE & & & & N48 & 08 \\
\hline TLC & & & & N44 & 30 \\
\hline $\mathrm{FE}$ & & cu & NI CO & N48 & 47 \\
\hline$A U$ & FE & & & N51 & 37 \\
\hline$A U$ & & $A G$ & & N51 & 06 \\
\hline PB & $\mathrm{ZN}$ & $\mathrm{cu}$ & & N48 & 38 \\
\hline
\end{tabular}

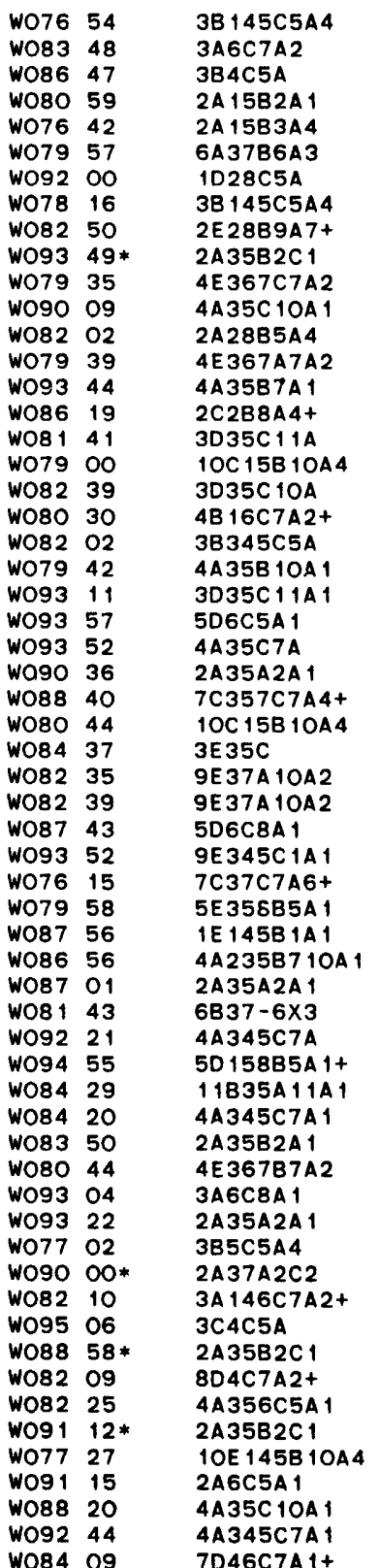




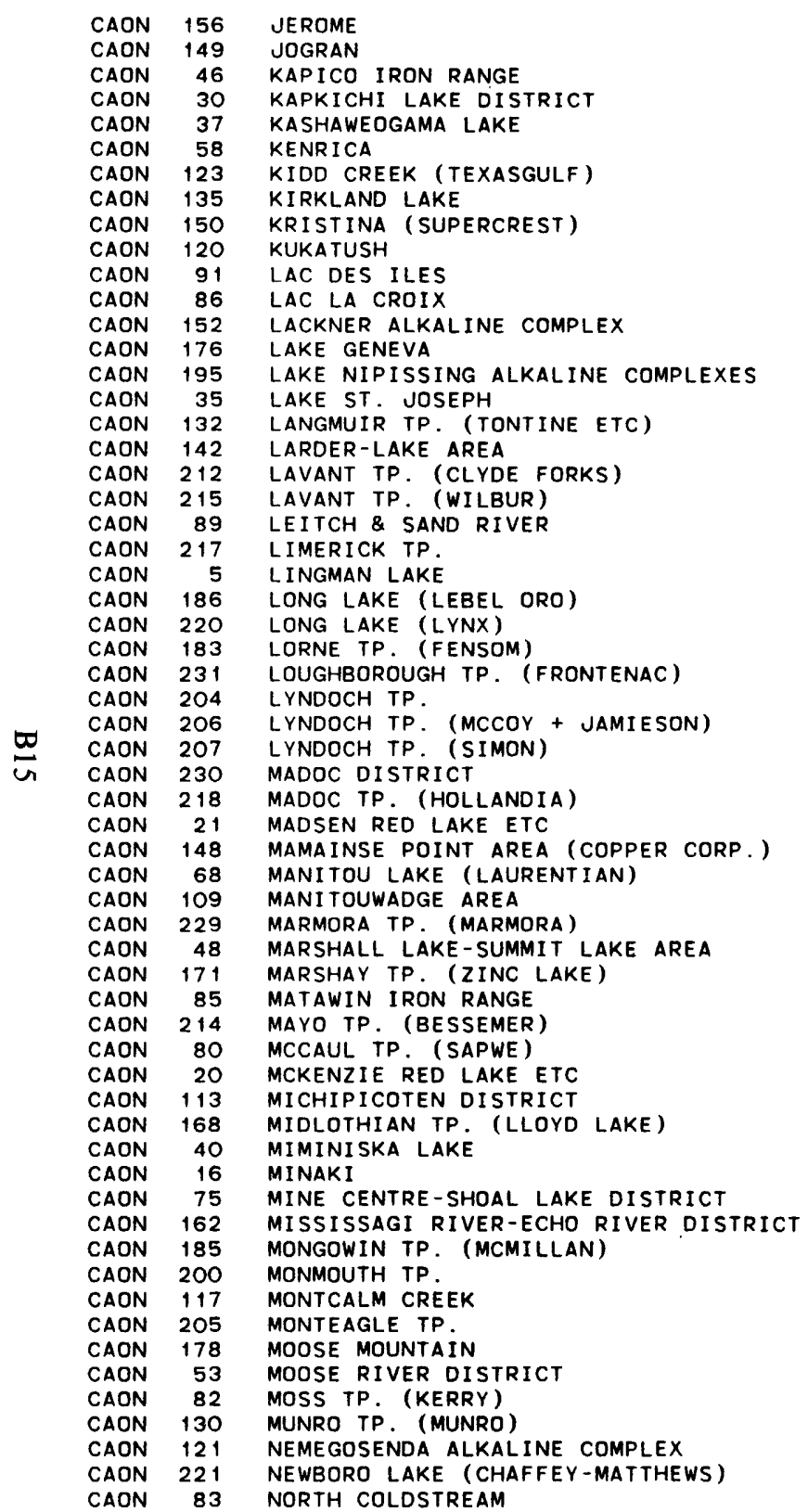

\begin{tabular}{|c|c|c|c|c|c|c|c|c|c|}
\hline \multirow{3}{*}{$\begin{array}{l}\mathrm{AU} \\
\mathrm{CU} \\
\mathrm{FE}\end{array}$} & \multirow{3}{*}{ MO } & & & AG & \multirow{2}{*}{$\begin{array}{l}\text { N47 } \\
\text { N47 }\end{array}$} & \multirow{2}{*}{$\begin{array}{l}37 \\
02\end{array}$} & \multirow{3}{*}{$\begin{array}{l}\text { WO82 } \\
\text { WO84 }\end{array}$} & \multirow{3}{*}{$\begin{array}{l}14 \\
37 \\
58\end{array}$} & \multirow{2}{*}{$\begin{array}{l}4 A 345 C 7 A 1 \\
3 C 4 C 5 A\end{array}$} \\
\hline & & & & & & & & & \\
\hline & & & & & & 41 & & & 2A35A2A1 \\
\hline $\mathrm{cu}$ & NI & & & & N5 1 & 30 & WO90 & 20 & 506B5A 1 \\
\hline FE & & & & & N50 & 25 & w090 & 41 & 2A35B2A 1 \\
\hline$A U$ & & & & & N49 & 43 & W094 & 40 & 4A35C7A \\
\hline $\mathrm{ZN}$ & $\mathrm{Cu}$ & $\mathbf{A G}$ & FES & PB & N48 & 42 & W08 1 & 22 & 3035A11A1 \\
\hline$A U$ & & & & $A G$ & N48 & 09 & W080 & 03 & 4A235A7A1+ \\
\hline $\mathrm{CU}$ & & & & & N46 & 53 & W084 & 05 & 3A4C7A \\
\hline FE & & & & & N48 & 09 & W082 & 14 & 2A35B2A1 \\
\hline PO & PT & & & NI $\mathrm{CU}$ & N49 & 10 & W089 & 37 & $5 \mathrm{C} 6 \mathrm{C} \mathrm{A}_{1}$ \\
\hline LI & & & & & N48 & 21 & W091 & 59 & IE $145 B 1 A 4$ \\
\hline NB & FE & P & U TH & REE & N47 & 47 & W083 & 08 & 1028A5A4 \\
\hline $\mathbf{Z N}$ & $\mathrm{PB}$ & & & $A G$ & N46 & 47 & W08 1 & 31 & $7 C 35 \mathrm{C} 7 \mathrm{~A}$ \\
\hline NB & $u$ & & & & N46 & 16 & W079 & 34 & 1028B5A6 \\
\hline $\mathrm{FE}$ & & & & & N50 & 58 & W09 1 & 03 & 2A35A2A1 \\
\hline NI & & & & $\mathrm{cu}$ & N48 & 21 & W08 1 & 01 & 5В358B5A 1 \\
\hline$A U$ & & & & $\mathbf{A G}$ & N48 & 08 & W079 & 35 & 4АЗ5B5A 1 \\
\hline cu & SB & $\mathbf{A G}$ & HG & & N45 & 07 & W076 & 43 & $3 A+C 10 A 4+$ \\
\hline $\mathrm{FE}$ & & & & & N45 & 01 & W076 & 41 & $2 A 145 C 3 A 4$ \\
\hline$A U$ & & & & $A G$ & N49 & 37 & W088 & 01 & 4A35B7A 1 \\
\hline NI & $\mathrm{cu}$ & & & & N44 & 51 & W077 & 43 & 5D 156C5A4 \\
\hline AU & & & & & N53 & 52 & W092 & 53 & $4 A 35 C 7 A$ \\
\hline AU & & & & & N46 & 18 & W081 & 09 & $4 A 146 C 5 A 3-4$ \\
\hline $\mathrm{ZN}$ & PB & & & & N44 & 41 & WO76 & 46 & $7 B 1 C 10 A 4+$ \\
\hline $\mathrm{ZN}$ & $\mathrm{CU}$ & FES & & & N46 & 17 & W08 1 & 33 & $301 C 7 A 2+$ \\
\hline PB & & & & $Z N A G$ & N44 & 28 & WO76 & 31 & 7A1C7A4+ \\
\hline $\mathrm{BE}$ & & & & & N45 & 20 & W077 & 24 & IC $145 C$ C IA4 \\
\hline MO & & & & & N45 & 16 & W077 & 28 & $3 B 45 C 5 A 4$ \\
\hline cu & $Z N$ & FES & & & N45 & 12 & W077 & 19 & $3 D 5 C$ \\
\hline $\mathbf{F}$ & & & & & N44 & 29 & W077 & 28 & $8 C 37 B 7 A 6+$ \\
\hline PB & & & & & N44 & 40 & W077 & 33 & $7 A 5 C 7 A 4+$ \\
\hline$A U$ & & & & AG & N5O & 58 & W093 & 55 & 4A35B7A 1 \\
\hline CU & & & & & N47 & 01 & W084 & 45 & 3A37C7A4 \\
\hline$A U$ & & & & & N49 & 27 & w092 & 42 & $4 A 35 C 7 A$. \\
\hline $\mathbf{Z N}$ & CU & $\mathbf{A G}$ & FES & PB AU & N49 & 09 & W085 & 47 & $3035 A 1 \mid A 1$ \\
\hline $\mathrm{FE}$ & & & & & N44 & 29 & W077 & 39 & $2 A 125 B 3 A 4$ \\
\hline $\mathrm{ZN}$ & $\mathrm{CU}$ & $\Delta G$ & & & N5O & 24 & W087 & 37 & $3 D 35 C 11 A 1$ \\
\hline $\mathrm{ZN}$ & PB & & & $A G$ & N47 & 09 & W08 1 & 27 & 7C35C 1OA \\
\hline $\mathrm{FE}$ & & & & & N48 & 33 & W090 & $05 *$ & 2A35B2C1 \\
\hline FE & & & & & N45 & 03 & W077 & 38 & $2 A 145 C 3 A 4$ \\
\hline$A U$ & & & & & N48 & 47 & W09 1 & 24 & $4 A 345 C 7 A$ \\
\hline$A U$ & & & & $A G$ & N5 1 & 04 & w093 & 50 & $\triangle A 4 C 7 A 1$ \\
\hline $\mathrm{FE}$ & & & & & N48 & 07 & W084 & 38 & 2A35A2A 1 \\
\hline ASB & & & & & N47 & 53 & W08 1 & $\infty 0$ & 5E8B5A \\
\hline FES & & & & & N5 1 & 37 & WO88 & $35 *$ & 2A35A2C1 \\
\hline FES & & & & & N5O & 04 & W094 & 36 & $11 B 35 B 11 A$ \\
\hline$A U$ & & & & & N48. & 44 & W092 & 37 & 4A345C7AI \\
\hline $\mathrm{CU}$ & & & & & N46 & 25 & W083 & 30 & $3 A 367 C 7 A 2$ \\
\hline$A U$ & & & & & N46 & 08 & W08 1 & 48 & $4 A 16 C 7 A 2+$ \\
\hline$u$ & & & & & N44 & 55 & W078 & 19 & 9A $145 C 3 A 4$ \\
\hline NI & $\mathrm{CU}$ & & & & N48 & 40 & W08 2 & 06 & 50356B5A 1 \\
\hline GRF & & & & & N45 & 13 & W077 & 52 & TIE IC TOA 4 \\
\hline FE & & & & & N46 & 51 & W08 1 & 02 & 2A35B2A1 \\
\hline GYP & & & & & N50 & 51 & W08 1 & 17 & 6АЗ7А6A3 \\
\hline$A U$ & AG & & & & N48 & 32 & W090 & 47 & $4 A 345 C 7 A 1$ \\
\hline ASB & & & & & N48 & 33 & W080 & 15 & 5Е $358 B 5 A 1$ \\
\hline NB & FE & $\mathbf{P}$ & U TH & REE & N48 & $\infty$ & W083 & 04 & $1028 A 5 A 4$ \\
\hline $\mathrm{FE}$ & TI & & & & N44 & 38 & W076 & 20 & 2C68B8A4 \\
\hline $\mathrm{CU}$ & & & & $A U A G$ & N48 & 36 & W090 & 35 & $3 A 356 C 5 A 1$ \\
\hline
\end{tabular}




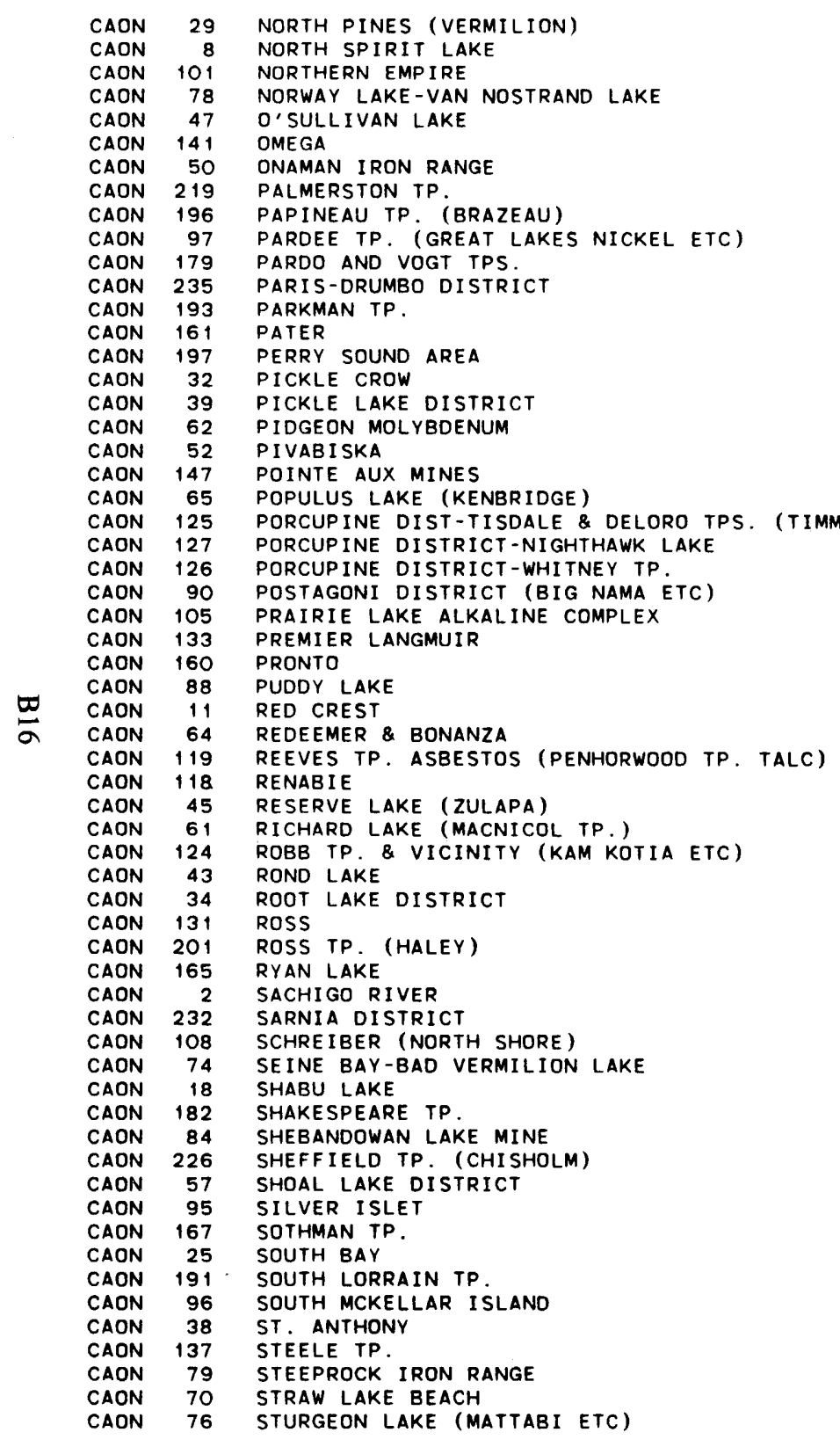

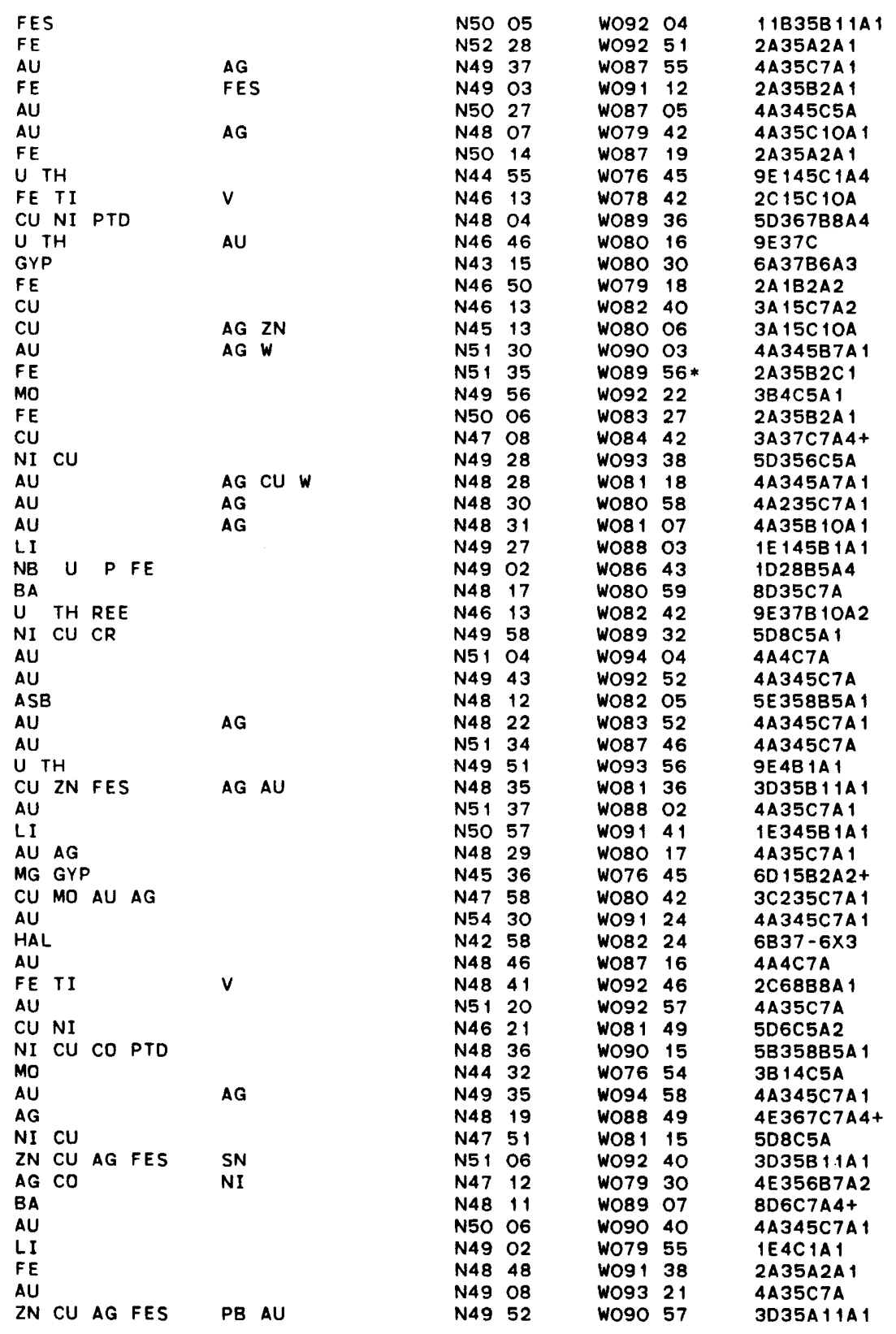




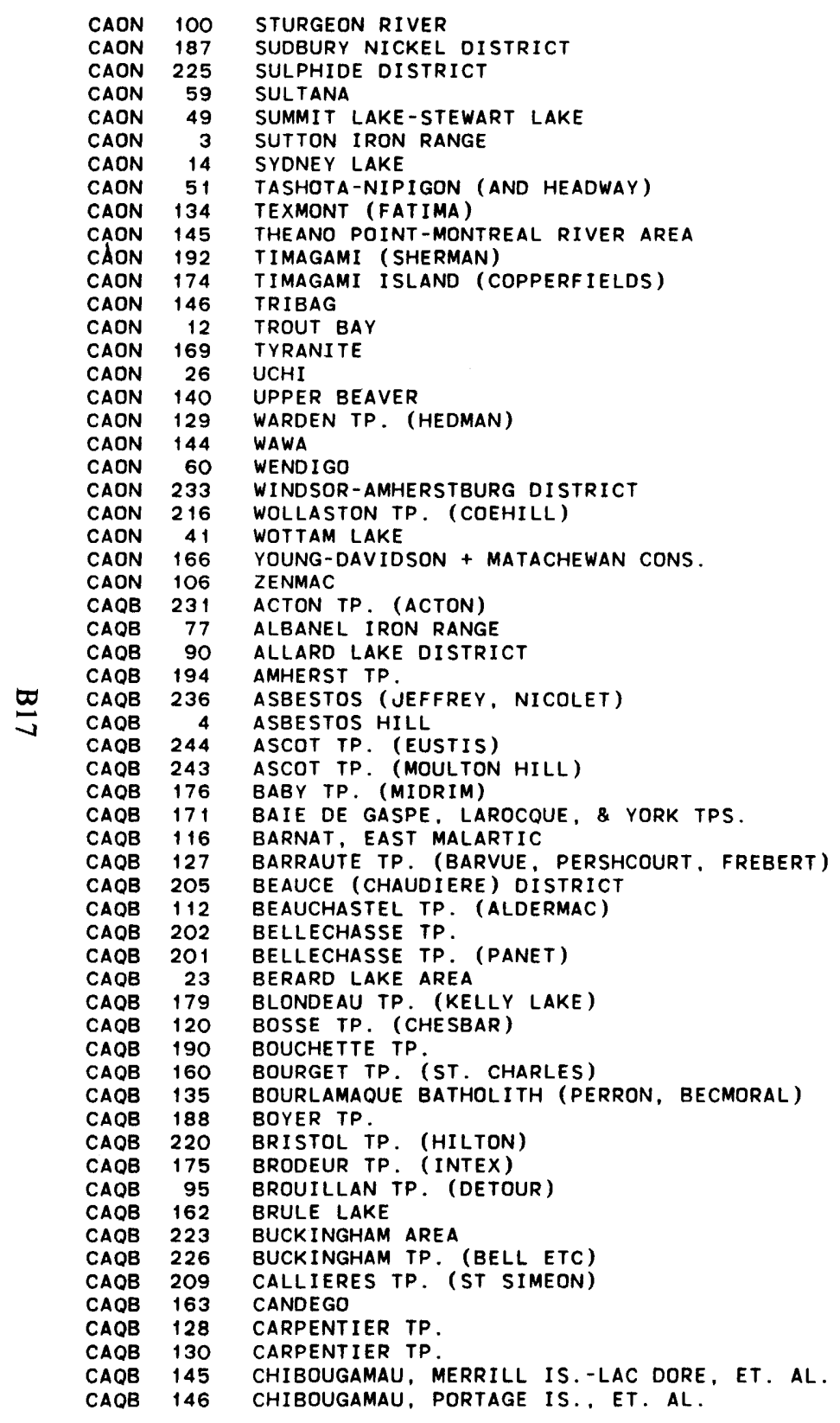

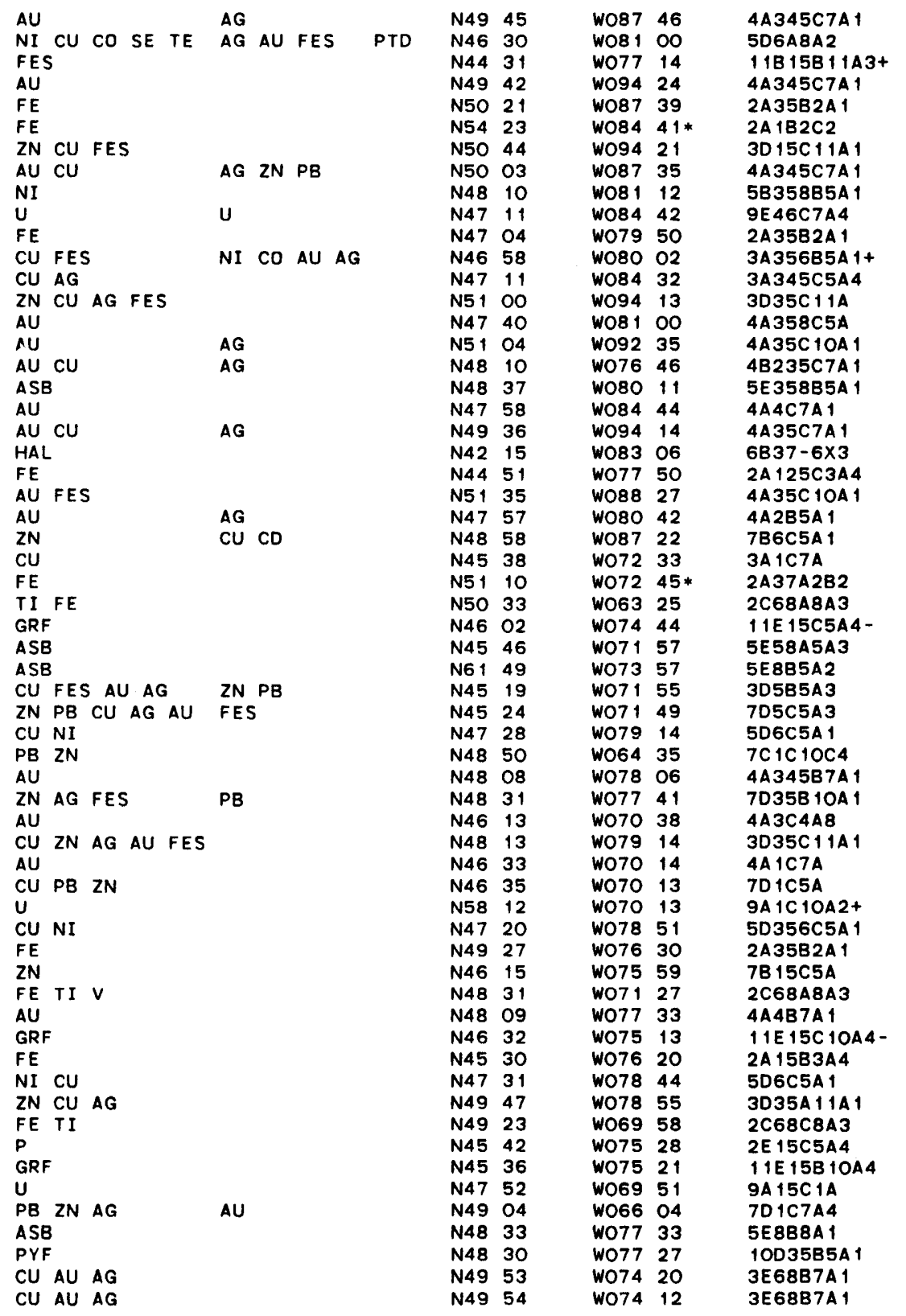




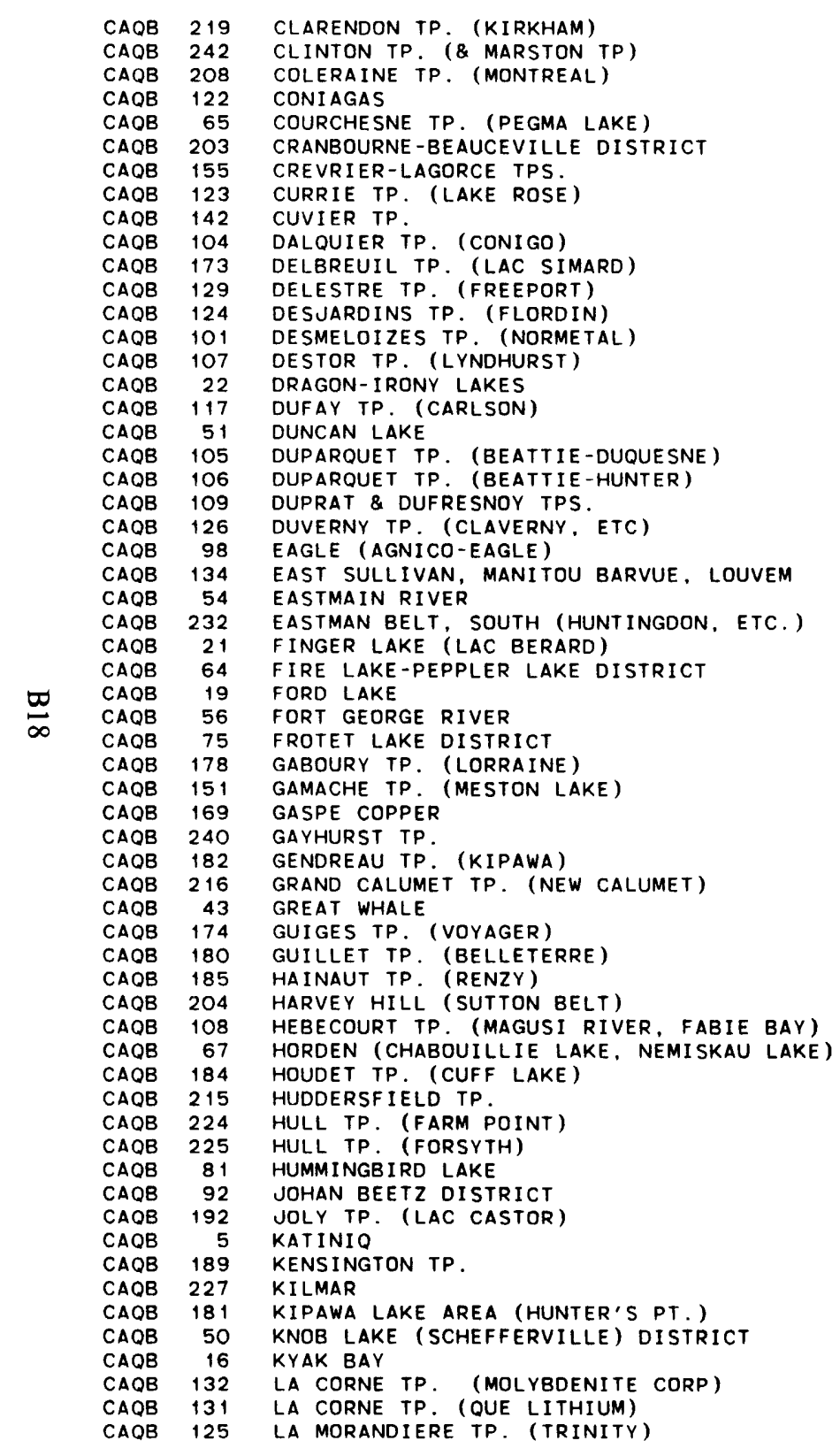

\begin{tabular}{|c|c|c|c|c|c|c|c|c|c|}
\hline мо & & & & & N45 & 41 & WO76 & 24 & $3 B 15 C 3 A 4$ \\
\hline $\mathrm{Cu}$ & $\mathrm{ZN}$ & FE & & PB $\quad A G$ & N45 & 27 & WO70 & 54 & $30 C+1 A$ \\
\hline$C R$ & & & & & N46 & $\infty$ & WO71 & 15 & $5 А 58 C 5 A 3$ \\
\hline $\mathrm{ZN}$ & AG & PB & & & $\mathrm{N} 49$ & 30 & WO76 & 10 & 7D35B $11 A 1$ \\
\hline cu 1 & NI & & & & N52 & 22 & W066 & 46 & $5 D 156 C 8 A$ \\
\hline ASB & & & & & N46 & 23 & W070 & $38 *$ & $5 \mathrm{E} 58 \mathrm{~B} 5 \mathrm{~B} 3$ \\
\hline NB & TA & $U \mathrm{ZR}$ & $R \quad P$ & & N49 & 29 & WO72 & 48 & 1028B5A \\
\hline AU & & & & & N49 & 22 & W076 & 49 & $4 A 35 C 7 A 1$ \\
\hline $\mathrm{CU}$ & $\mathrm{ZN}$ & FES & & & N49 & 57 & WO74 & 53 & $3035 C_{10 A} 1$ \\
\hline $\mathrm{CU}$ & & & & ZN & N48 & 36 & WO78 & 03 & 3A $35 C 10 A 1$ \\
\hline LI & & & & & N47 & 40 & WO78 & 39 & IEAC $1 A 1$ \\
\hline FES & & & & & N48 & 38 & WO77 & 02 & 11B35B $11 A 1$ \\
\hline AU & & & & & N49 & 18 & W076 & 55 & $4 A 35 C 7 A 1$ \\
\hline $\mathrm{cu}$ & $\mathrm{ZN}$ & $A G A U$ & $J$ FES & & N49 & $\infty$ & W079 & 22 & 3D $35 B 11 A 1$ \\
\hline $\mathrm{cu}$ & $A U$ & & & & N48 & 34 & WO78 & 57 & उE $35 \mathrm{C} 10 \mathrm{AA}$ \\
\hline FE & & & & MN & N58 & 19 & W070 & $15 *$ & $2 A 1 A 2 C 2$ \\
\hline$c U$ & & & & & N48 & 08 & WO79 & 27 & $3 \mathrm{~A}_{3} \mathrm{C} 7 \mathrm{~A}_{1}$ \\
\hline $\mathrm{FE}$ & & & & & N53 & 30 & W077 & 51 & 2A35A2A1 \\
\hline $\mathrm{AU}$ & AG & AS & & & N48 & 30 & WO79 & 14 & $\triangle A 4 B 5 A 1$ \\
\hline $\mathrm{CU}$ & & & & & N48 & 33 & WO79 & 08 & $3 A 35 C 10 A 1$ \\
\hline $\mathbf{Z N}$ & $\mathrm{Cu}$ & $A G \quad A U$ & J FES & & N48 & 21 & WO79 & 05 & 3D $35 A 11 A 1$ \\
\hline AU & & & & $\mathrm{CU}$ & N48 & 39 & W077 & 54 & AA4C7A1 \\
\hline$A U$ & FES & & & & N49 & 29 & WO78 & 21 & 4A35B 10A 1 \\
\hline CU & $\mathrm{ZN}$ & $A G \quad A U$ & $\int C D$ & FES PB & N48 & 05 & W077 & 40 & 3D35B $11 A 1$ \\
\hline L I & & & & & N52 & 18 & W077 & 03 & $1 E 345 B 1 A 1$ \\
\hline$C U$ & & & & $Z N A U A G$ & N45 & 15 & WO72 & 20 & $3 А 58 C 5 A 3$ \\
\hline $\mathrm{FE}$ & & & & & N58 & 41 & WO70 & $04 *$ & $2 A 1 A 2 C 2$ \\
\hline FE & & & & & N52 & 21 & W067 & 22 & 2A 15A2A 2 \\
\hline FE & & & & & N59 & 13 & WO70 & $10 *$ & $2 A 1 A 2 C 2$ \\
\hline $\mathrm{FE}$ & & & & & N53 & 44 & WO75 & 51 & $2 A 3 C 4 A 8$ \\
\hline $\mathrm{CU}$ & $\mathrm{ZN}$ & & & & N5O & 38 & WO74 & 38 & 3D $35 \mathrm{C} \mathrm{A}_{4}$ \\
\hline CU 1 & NI & & & & N4 7 & 21 & WO78 & 56 & 5D356C5A 1 \\
\hline AU & & & & & N49 & 29 & WO74 & 30 & 4A4C5A1 \\
\hline CU 1 & MO & BI & & & N48 & 57 & WO65 & 31 & 3A14A3A5 \\
\hline MO & & & & & N45 & 46 & W070 & 54 & 3B 45C7A4 \\
\hline $\mathrm{FE}$ & & & & & N46 & 48 & WO79 & - & $2 A+5 C 5 A$ \\
\hline $\mathrm{ZN}$ & PB & $A G \quad A U$ & & & N45 & 42 & WO76 & 41 & 7D 15B5A4 \\
\hline $\mathrm{FE}$ & & & & & N55 & 05 & W076 & 50 & 2A35A2A1 \\
\hline FE & & & & & N47 & 34 & WO79 & $20 *$ & $2 A 35 B 2 C 1$ \\
\hline$A U$ & & & & AG & N47 & 24 & W078 & 41 & $4 A 35 C 7 A 1$ \\
\hline cu 1 & NI & & & & N46 & 48 & W076 & 42 & 5D8C8A \\
\hline $\mathrm{CU}$ & & & & & N46 & 16 & WO71 & 12 & 3A 1C5A \\
\hline $\mathrm{ZN}$ & cu & $A G$ & & & N48 & 26 & WO79 & 22 & 3D35B 1 १A 1 \\
\hline $\mathrm{CU}$ & NI & & & & N5O & 56 & WO77 & 48 & 5D6B5A \\
\hline FE & & & & & N47 & 01 & WO77 & 07 & $2 A 15 C 2 A$ \\
\hline TH & $u$ & & & & N45 & 57 & WO76 & 33 & 9E $145 C 3 A 4$ \\
\hline MG & & & & & N45 & 36 & WO75 & 54 & 6D 15C 1OA4 \\
\hline $\mathrm{FE}$ & & & & & N45 & 28 & WO75 & 47 & 2A 15C $3 A 4$ \\
\hline $\mathrm{FE}$ & & & & & N51 & 45 & W069 & 33 & 2A15B2A2 \\
\hline$u$ & & & & TH & N50 & 25 & W062 2 & 53 & SE 4C 1A4 \\
\hline GRF & & & & & N46 & 16 & WO74 & 41 & 11E 15C5A4 - \\
\hline NI & & & & $\mathrm{cu}$ & N6 1 & 40 & WO73 & 40 & 5B8B8A2 \\
\hline MO & & & & & N46 & 24 & W075 & 52 & $3 B 4 C 1 A$ \\
\hline MG & & & & & N45 & 45 & W074 & 37 & 6D15A5A4 \\
\hline u & $A U$ & & & & N46 & 59 & W078 & 47 & 9A 15C $10 A 2+$ \\
\hline FE & & & & & N54 & 50 & W066 & 54* & $2 A 1 A 2 B 2$ \\
\hline $\mathrm{cu}$ & NI & & & & N60 & 08 & W069 & 53 & 5D6C5A2 \\
\hline MO & BI & & & BE & N48 & 17 & W077 & 59 & 3B4B $1 \mathrm{~A} 1$ \\
\hline LI & & & & & N48 & 24 & W077 & 48 & IE $4 A \mid A 1$ \\
\hline $\mathrm{cu}$ & $\mathrm{ZN}$ & FES & & & N48 & 42 & W077 & 45 & 3D35C $11 A 1$ \\
\hline
\end{tabular}




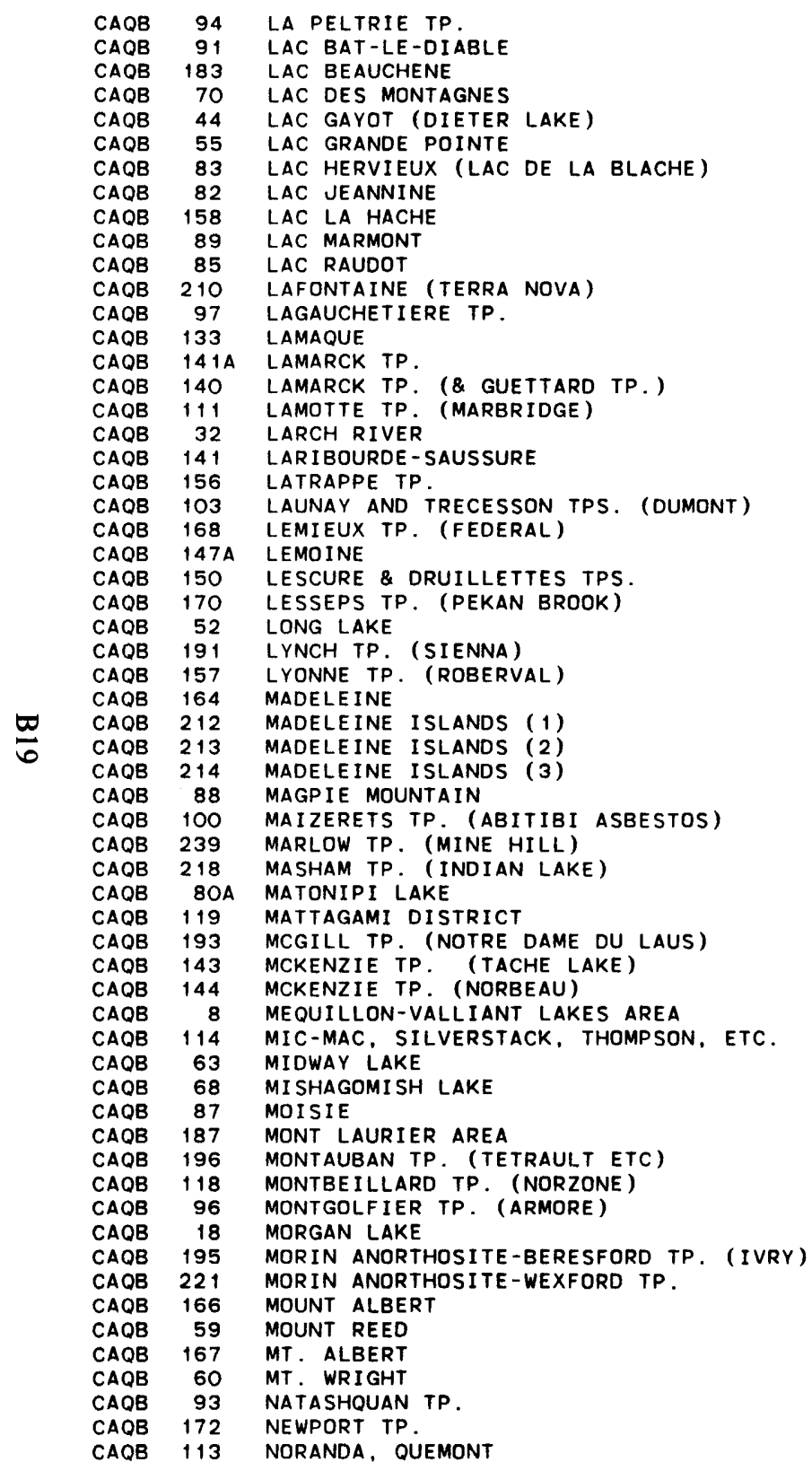

\begin{tabular}{|c|c|c|c|c|c|c|c|c|}
\hline CU & $Z N$ & & & N49 & 55 & W079 & 15 & 3D35C5A 1 \\
\hline TI & $\mathrm{FE}$ & & & N5O & 27 & W063 & 40 & $2 C 68 C 8 A 3$ \\
\hline KYN & & & & N46 & 33 & W079 & $\infty$ & 1OC 15B $10 A 4$ \\
\hline LI & & & & N5 1 & 40 & W075 & 50 & $1 E 345 C 1 A 1$ \\
\hline$u$ & & & & N55 & 59 & W070 & 34 & 9A37B $10 A 2$ \\
\hline FE & & & & N53 & 54 & W075 & $42 *$ & $2 \mathrm{~A} 35 \mathrm{C} 2 \mathrm{C} 1$ \\
\hline FE & TI & & & N5O & 03 & W069 & 38 & 2C68ABA3 \\
\hline FE & & & & N5 1 & 51 & W068 & 06 & 2A15A2A2 \\
\hline FE & TI & $\mathbf{P}$ & & N48 & 57 & W070 & 56 & 2С $4 B 8 A 3$ \\
\hline FE & & & TI & N5O & 46 & W065 & 19 & $2 C 15 C$ \\
\hline$F E$ & TI & & & N5 1 & 42 & W067 & 45 & $2 \mathrm{C} 6 \mathrm{CBA} 3$ \\
\hline$A S B$ & & & & N47 & 05 & W069 & 47 & 5E58C5A3 \\
\hline $\mathrm{ZN}$ & $\mathrm{CU}$ & $\mathbf{A G}$ & & N49 & 46 & W078 & 09 & $3 D 35 B+1 A 1$ \\
\hline$A U$ & & & & N48 & 06 & W077 & 45 & 4A 4B7A 1 \\
\hline FES & & & & N49 & 56 & W075 & 15 & $11835 C 11 A 1$ \\
\hline $\mathrm{cU}$ & NI & & & N49 & 59 & WO75 & 18 & $506 C 8 A 2$ \\
\hline NI & & & & N48 & 21 & WO78 & 11 & $5 B 358 C 5 A 1$ \\
\hline FE & MN & & & N57 & 33 & WO70 & $07 *$ & $2 A 1 A 2 C 2$ \\
\hline$Z N$ & $\mathrm{CU}$ & $\mathbf{A G}$ & & N49 & 48 & WO75 & 32 & $3 D 35 C 11 A 1$ \\
\hline MG & & & & N49 & 05 & W072 & 10 & $6 D 15 C 10 A 3$ \\
\hline NI & & & & N48 & 39 & WO78 & 26 & 5B8B8A 1 \\
\hline $\mathrm{ZN}$ & PB & & cu & N48 & 46 & W066 & 08 & 7D $1 C 7 A 5$ \\
\hline $\mathrm{ZN}$ & $\mathrm{cu}$ & $\triangle G A U$ & & N49 & 46 & W074 & 06 & 3D35B 1 $1 A 1$ \\
\hline $\mathrm{cu}$ & FES & & & N49 & 30 & WO74 & 54 & $3 A 35 C 10 A 1$ \\
\hline CU & & & $\mathrm{ZN}$ & N48 & 52 & W065 & 58 & 3A 1 C3A5 \\
\hline $\mathrm{CU}$ & & & & N53 & 35 & W077 & 19 & $3 A 35 C 11 A 1$ \\
\hline FE & & & & N46 & 16 & W074 & 49 & $2 A 37 C 2 A$ \\
\hline $\mathrm{FE}$ & TI & $C R$ & & N48 & 28 & WO72 & 42 & 2С68АВАЗ \\
\hline $\mathrm{CU}$ & & & $A G$ & N49 & $\infty$ & W066 & $\infty$ & 3А 1B3A4 \\
\hline HAL & & & $\mathrm{k}$ & N47 & 37 & W06 1 & 33 & 6B3A6A5 \\
\hline MN & & & & N47 & 25 & WO6 1 & 45 & $10 A 37 C 5$ \\
\hline GYP & & & & N47 & 23 & W06 1 & 52 & 6A3C6A5 \\
\hline FE & TI & & CR & N5 1 & 25 & W064 & 03 & 2C68ABA3 \\
\hline ASB & & & & N49 & 11 & W078 & 02 & 5E 8B5A 1 \\
\hline PB & $\mathbf{A G}$ & w & $A U$ & N45 & 46 & W070 & 31 & 4D17C7A4 \\
\hline MO & & & & N45 & 44 & W076 & 08 & ЗВ 15С3А4 \\
\hline FE & & & & N5 1 & 52 & W069 & $50 *$ & $2 A 15 B 2 C 2$ \\
\hline CU & $Z N$ & $A G \quad A U$ FES & NI & N49 & 43 & W077 & 43 & $3035 A 11 A 1$ \\
\hline GRF & & & & N46 & 08 & W075 & 33 & 11E 15B5A4 - \\
\hline $\mathbf{Z N}$ & $A U$ & $A G$ & & N49 & 56 & W074 & 24 & $70358 C 7 A$ \\
\hline$A U$ & & & & N49 & 58 & W074 & 18 & 4A6C7A1 \\
\hline CU & NI & & & N61 1 & 30 & W073 & 45 & 5D68C8A2 \\
\hline AU & cu & & & N48 & 16 & W078 & 33 & AB35B 1OA 1 \\
\hline $\mathrm{FE}$ & & & & N52 & 28 & W067 & OO* & $2 A 15 C 2 C 2$ \\
\hline FE & & & & N5O & 53 & WO76 & $21 *$ & $2 A 35 C 2 C 1$ \\
\hline FE & $T I$ & & & N5O & 13 & W066 & 05 & $2 C 37 C 4 A B$ \\
\hline$U T$ & $\mathrm{rH}$ & & & N46 & 53 & W075 & 20 & $9 E 15 C 1 A$ \\
\hline $\mathrm{ZN}$ & PB & $A U A G$ & & N46 & 49 & W072 & 21 & 7D 15B 10A \\
\hline $2 \mathrm{~N}$ & PB & & & N48 & 08 & W079 & 11 & 7C35C7A1 \\
\hline FE & & & & N49 & 43 & W078 & 37 & 2A35A2A1 \\
\hline FE & & & & N59 & 44 & W070 & $00 *$ & $2 A 1 A 2 C 2$ \\
\hline FE & TI & & & N46 & 05 & W074 & 21 & $2 C 68 C 8 A 3$ \\
\hline FE & TI & & & N45 & 58 & W074 & 02 & 2C68A8A3 \\
\hline NI & & & & N48 & 51 & W066 & 16 & $5 B 18 \mathrm{C} 10 \mathrm{A3}$ \\
\hline FE & & & & N52 & 01 & W068 & 05 & 2A15A2A2 \\
\hline CR & & & & N48 & 54 & W066 & 10 & $5 A 8 C 5 A 3$ \\
\hline FE & & & & N52 & 44 & W067 & 15 & 2A $15 A 2 A 2$ \\
\hline FE & TI & & & N50 & 05 & W06 1 & 42 & $2 C 37 C 4 A 8$ \\
\hline CU & & & & N48 & 17 & W064 & 44 & 3A5C 1OA \\
\hline $\mathrm{CU}$ & $A U$ & $Z N$ AG SE & TE FES & N48 & 15 & W079 & $\infty$ & 3E 35A $11 A 1$ \\
\hline
\end{tabular}




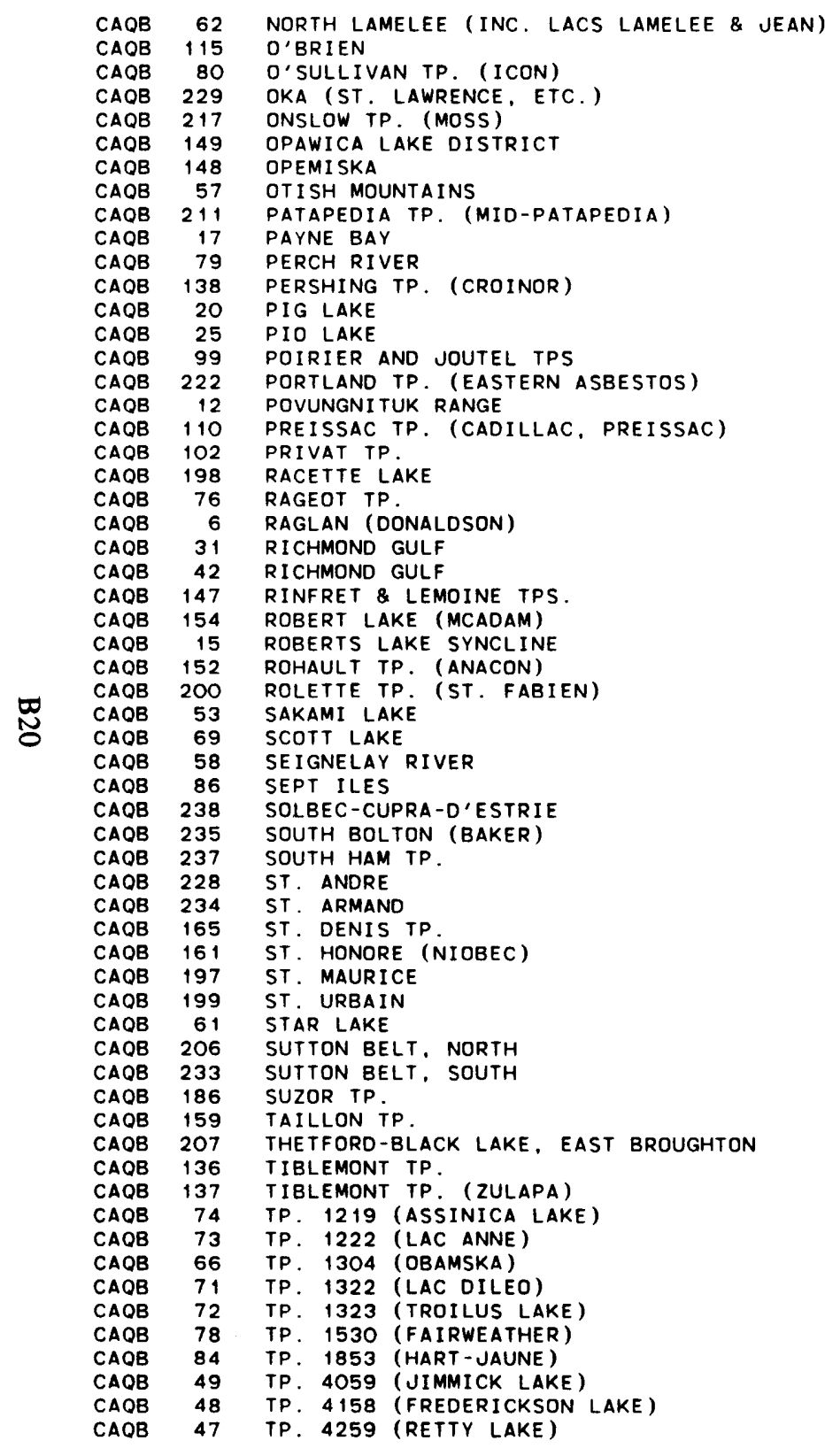

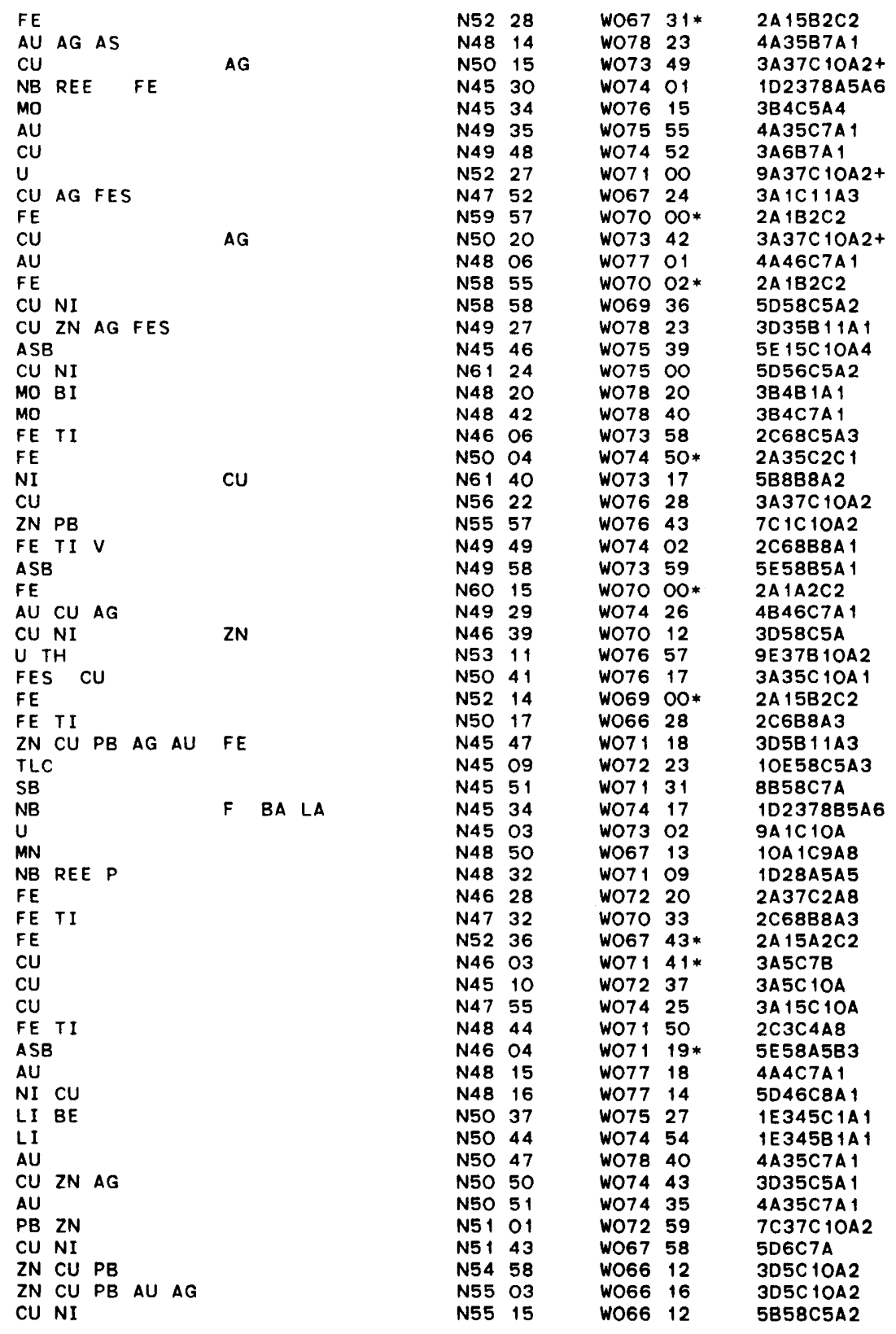




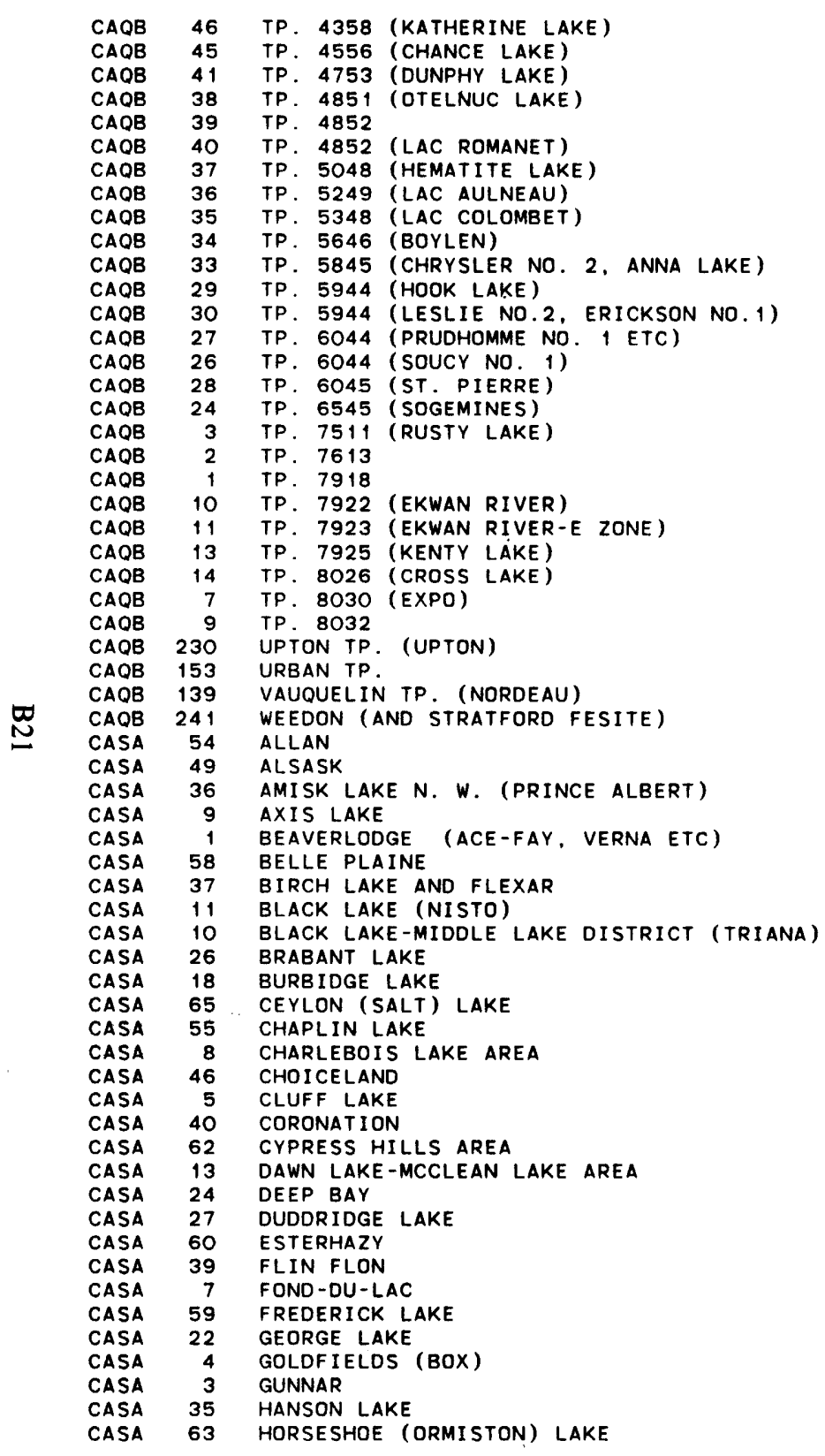

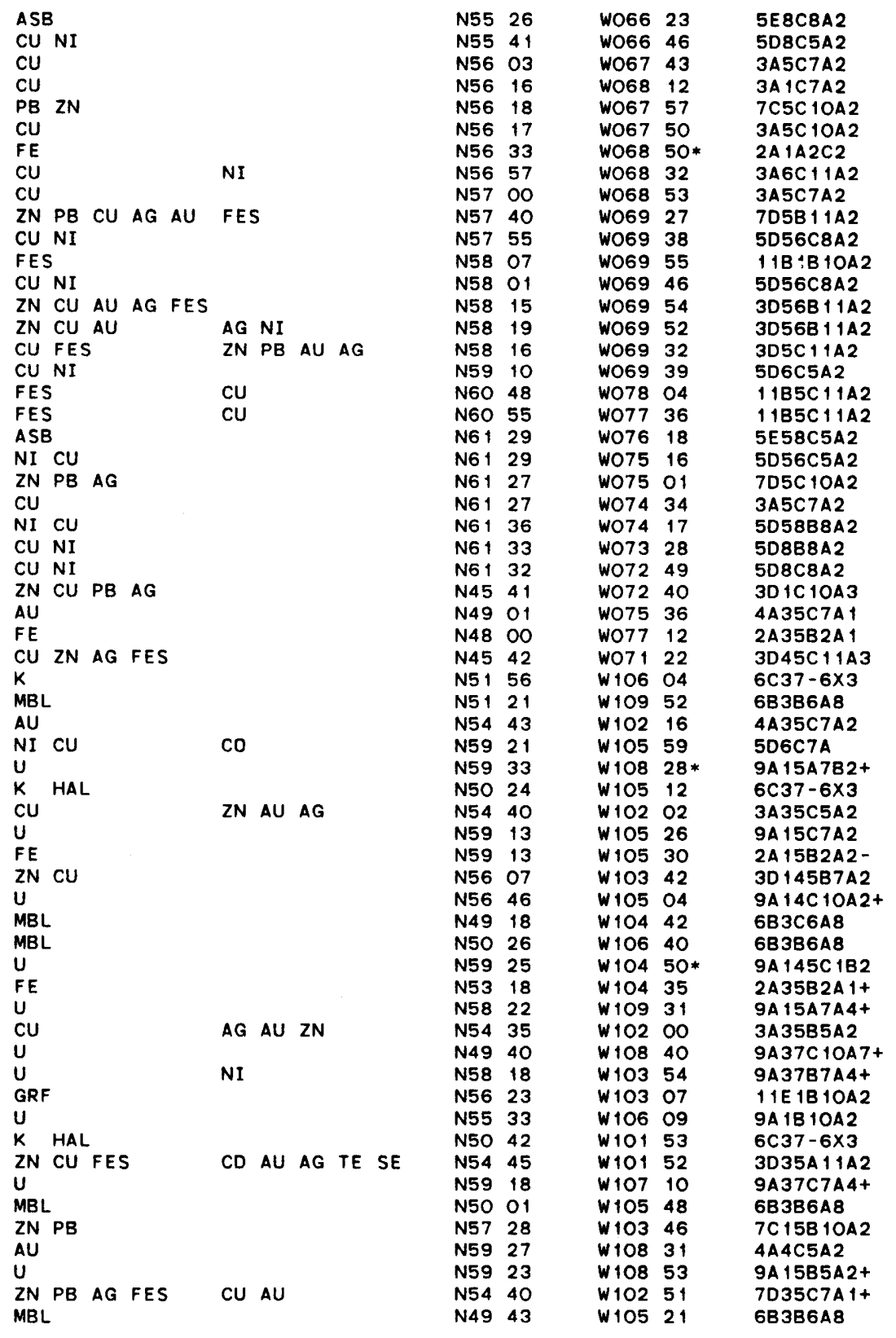




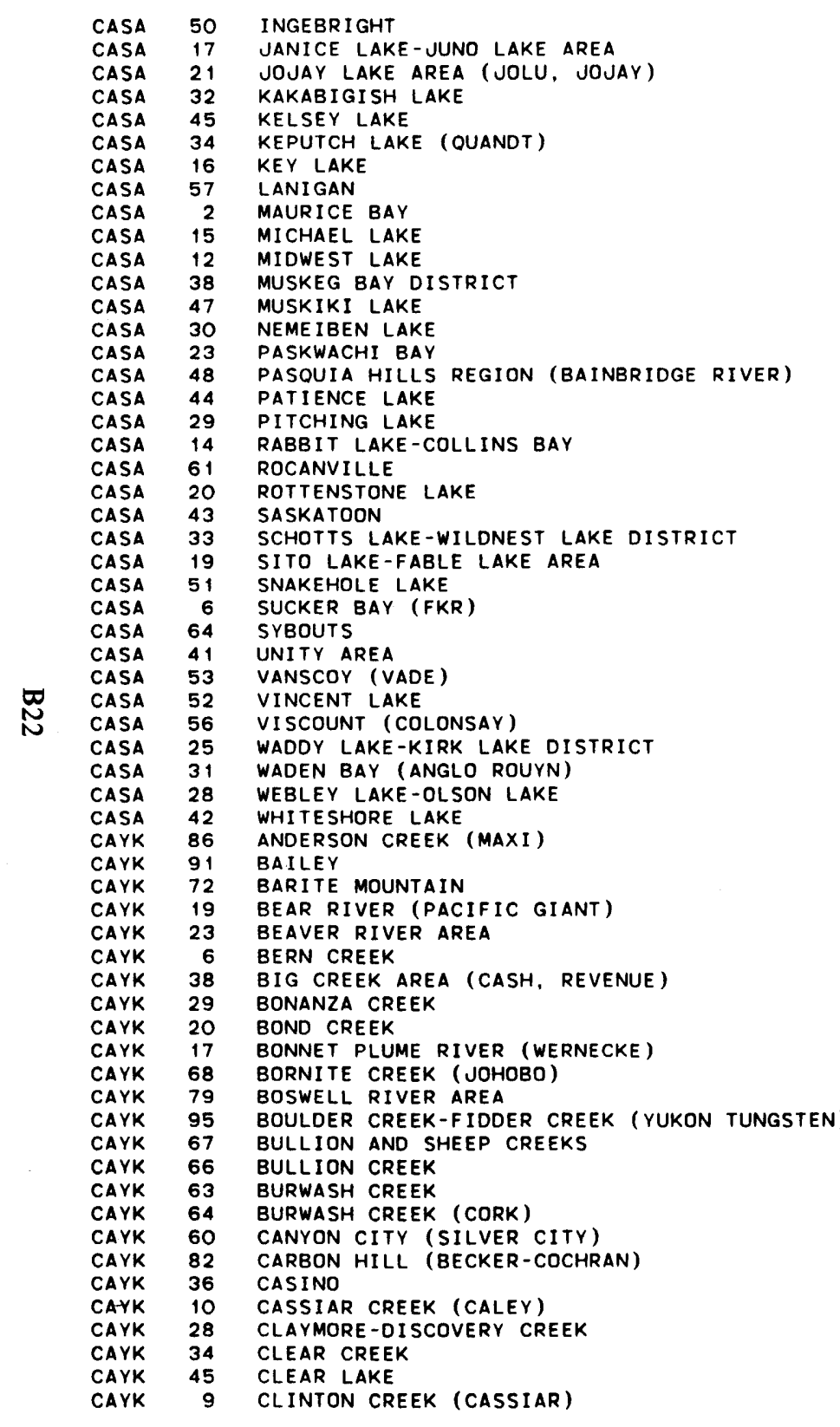

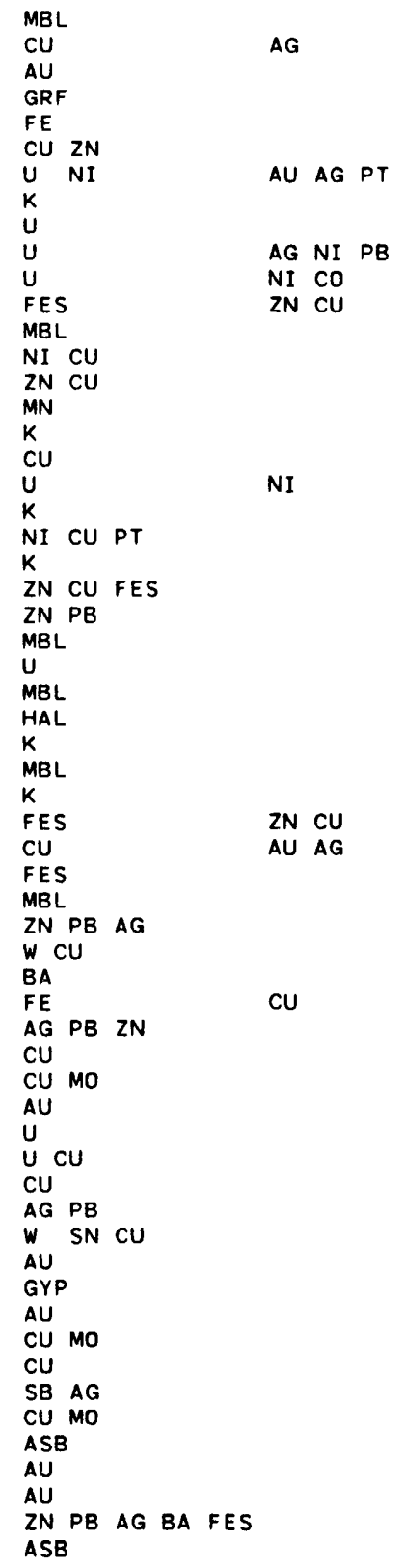

\begin{tabular}{|c|c|c|c|c|}
\hline 150 & 22 & $W 109$ & 19 & 6B3A6A8 \\
\hline N56 & 53 & W104 & 58 & $3 A 15 C 10 A 2$ \\
\hline N56 & 00 & W104 & 16 & $4 A 145 C 7 A$ \\
\hline N55 & 51 & W103 & 59 & 11E 15C 10A \\
\hline N53 & 35 & $W 104$ & 27 & 2A35B2A1+ \\
\hline N54 & 44 & W 102 & 45 & $30145 C 7 A 2$ \\
\hline N57 & 12 & W105 & 39 & 9A1A7A4+ \\
\hline N5 1 & 51 & W105 & 13 & $6 c 37-6 \times 3$ \\
\hline N59 & 23 & $W 109$ & 54 & 9A3787A4+ \\
\hline N57 & 52 & $W 104$ & 04 & 9A37B7A4+ \\
\hline N58 & 16 & $W 104$ & 07 & 9A37A7A4+ \\
\hline N54 & 34 & $W 102$ & 22 & 11B35B $11 \mathrm{~A} 2$ \\
\hline N52 & 19 & W105 & 44 & 6B3B6A8 \\
\hline N55 & 19 & W105 & 10 & $508 C 5 A$ \\
\hline N57 & 17 & $W 102$ & 01 & $30145 C_{10 A 2}-$ \\
\hline N53 & 33 & $w 102$ & 09 & $10 A 37 C 2 A 6$ \\
\hline N52 & 05 & $W 106$ & 23 & $6 c 37-6 \times 3$ \\
\hline N55 & 26 & W104 & 08 & $3 A 15 C 11 A 2$ \\
\hline N58 & 12 & W103 & 43 & 9A 1A5A4+ \\
\hline N50 & 28 & W101 & 33 & $6 c 37-6 \times 3$ \\
\hline N56 & 20 & $W 104$ & 49 & $50158 C 5 A 2$ \\
\hline N52 & 06 & W 106 & 51 & $6 c 37-6 \times 3$ \\
\hline N55 & 05 & W102 & 14 & $3035 C 11 A$ \\
\hline N56 & 15 & W105 & 40 & $7 C 1 C 10 A 2$ \\
\hline N50 & 31 & W 108 & 29 & 6B3B6A8 \\
\hline N59 & 18 & W106 & 37 & $9 A 145 C 7 A$ \\
\hline N49 & 02 & W104 & 25 & 6B3B6A8 \\
\hline N52 & 25 & W109 & 06 & 6B37A6A3 \\
\hline N52 & 00 & W 107 & 05 & $6 c 37-6 \times 3$ \\
\hline N50 & 13 & W108 & 57 & 6B3B6A8 \\
\hline N5 1 & 56 & W105 & 46 & $6 c 37-6 \times 3$ \\
\hline N56 & 12 & W104 & 00 & 11B35A11A2 \\
\hline N55 & 18 & W105 & oo & 3A 15C7A2 \\
\hline N55 & 55 & W105 & 27 & $11 B 15 C_{10 A 2}$ \\
\hline N52 & 07 & W108 & 17 & 6B3A6A8 \\
\hline N6 1 & 38 & $w 129$ & 11 & $7 D 1 C 10 A 2$ \\
\hline N60 & 46 & $W 128$ & 51 & 1A 14B3A5 \\
\hline N6 1 & 50 & $W 133$ & $\infty$ & $8 D 1 C 7 A 2+$ \\
\hline N64 & 50 & W134 & 17 & $2 A 1 C 5 A$ \\
\hline N64 & 25 & W135 & $20 *$ & 4D 16C7C1+ \\
\hline N66 & 09 & $W 140$ & 12 & 3A 1C7A \\
\hline N62 & 25 & $W 137$ & 36 & $3 C 4 C 5 A 4$ \\
\hline N63 & 57 & $w 139$ & 20 & 4A3B4A7 \\
\hline N64 & 40 & W134 & 55 & 9A5C5A1 \\
\hline N65 & 08 & W134 & 24 & 9A5C5A 1 \\
\hline N60 & 29 & $W 137$ & 34 & $3 A 5 C 7 A 3+$ \\
\hline N6 1 & 03 & $W 133$ & 48 & $40145 C 7 A 5$ \\
\hline N60 & 08 & $W 130$ & 26 & 1A14C7A4+ \\
\hline N6 1 & $\infty$ & W138 & 37 & 4A3C4A7+ \\
\hline N6 1 & 01 & $W 138$ & 42 & 6А5B5A3 \\
\hline N6 1 & 23 & $w 139$ & 15 & 4A3C4A8 \\
\hline N6 1 & 20 & $W 139$ & 30 & $3 C 45 C 5 A 7$ \\
\hline N6 1 & 47 & $W 140$ & 47 & $3 A 5 C 7 A 3+$ \\
\hline N6o & 11 & $W 135$ & 13 & 8B4C7A5+ \\
\hline N62 & 44 & $W 138$ & 49 & 3C4B5A6 \\
\hline N64 & 18 & $W 140$ & 13 & $5 E 58 C 8 A 3+$ \\
\hline N63 & 03 & $W 140$ & 56 & 4A3B4A7 \\
\hline N63 & 50 & W137 & 17 & $4 A 3 C 4 A 7+$ \\
\hline N62 & 48 & W135 & $\infty$ & $7 D 1 C 11 A 3$ \\
\hline N64 & 27 & W140 & 42 & 5E58B8A3+ \\
\hline
\end{tabular}




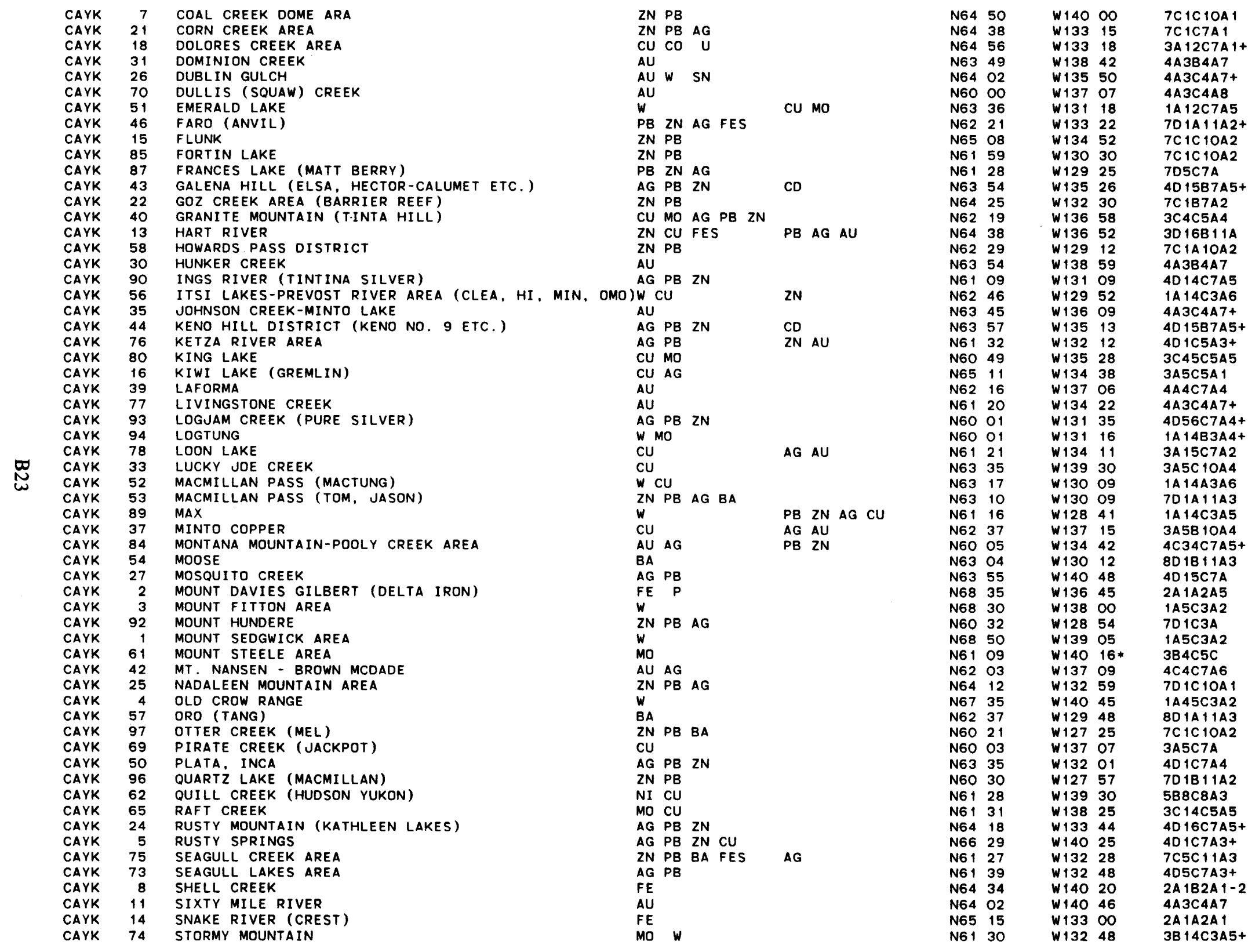




\begin{tabular}{|c|c|c|}
\hline CAYK & 32 & SULPHUR CREEK \\
\hline CAYK & 49 & SUNSET, SIR JOHN A. \\
\hline CAYK & 48 & SWIM LAKES AREA \\
\hline CAYK & 55 & TEA \\
\hline CAYK & 12 & TOMBSTONE MOUNTAIN \\
\hline CAYK & 71 & TWIN MOUNTAIN (RISBY) \\
\hline CAYK & 88 & TYERS RIVER AREA \\
\hline CAYK & 47 & VANGORDA CREEK (GRUM, ETC...) \\
\hline CAYK & 83 & WHEATON RIVER AREA \\
\hline CAYK & 59 & WHITE RIVER (CANALASK) \\
\hline CAYK & 81 & WHITEHORSE COPPER BELT (NEW IMPERIAL) \\
\hline CAYK & 41 & WILLIAMS CREEK \\
\hline $\operatorname{cscs}$ & 5 & ABANGARES DIST (TRES HERMANOS, BOSTON) \\
\hline $\operatorname{cscs}$ & 11 & AGUACATE DIST (OREAMUNO, CASTRO, ET AL.) \\
\hline $\operatorname{cscs}$ & 10 & CALDERA \\
\hline $\operatorname{cscs}$ & 16 & CARAIGRES \\
\hline $\operatorname{cscs}$ & 24 & CARATE \\
\hline $\operatorname{cscs}$ & 17 & CONCAVAS \\
\hline $\operatorname{cscs}$ & 22 & CORONADO \\
\hline $\operatorname{cscs}$ & 9 & ESPARTA DISTRICT \\
\hline $\operatorname{cscs}$ & 6 & GUACIMAL \\
\hline $\operatorname{cscs}$ & 18 & LA DIVISION \\
\hline $\operatorname{cscs}$ & 7 & MIRAMAR \\
\hline $\operatorname{cscs}$ & 1 & OCOTAL \\
\hline $\operatorname{cscs}$ & 23 & PLAYA MADRIGAL \\
\hline $\operatorname{cscs}$ & 2 & PLAYA REAL \\
\hline $\operatorname{cscs}$ & 4 & POAS \\
\hline $\operatorname{cscs}$ & 3 & POCO SOL \\
\hline $\operatorname{cscs}$ & 20 & PUERTO VIEJO \\
\hline $\operatorname{cscs}$ & 15 & PURI SCAL (SAN IGNACIO) PROSPECT \\
\hline $\operatorname{cscs}$ & 14 & RIO POAS \\
\hline $\operatorname{cscs}$ & 19 & SAN ISIDRO DEL GENERAL. \\
\hline $\operatorname{cscs}$ & 12 & SAN PABLO \\
\hline $\operatorname{cscs}$ & 8 & SAN RAMON \\
\hline $\operatorname{cscs}$ & 21 & UREN \\
\hline $\operatorname{cscs}$ & 13 & VILLA COLON \\
\hline cucu & $30 \mathrm{~A}$ & AMORES GROUP \\
\hline cucu & 14 & ANTONIO \\
\hline CUCU & 13 & BAEZ AREA \\
\hline cucu & 7 & BUENA VISTA ET AL \\
\hline CUCU & 19 & CAMAGUEY DISTRICT \\
\hline CUCU & 11 & CARLOTA MINE \\
\hline CUCU & 8 & CLARA MINE \\
\hline CUCU & 36 & DAIQUIRI-FIRMEZA DISTRICT \\
\hline CUCU & 3 & DORA \\
\hline CUCU & 33 & EL COBRE \\
\hline CUCU & 34 & ELECCION \\
\hline CUCU & 24 & EURECA \\
\hline CUCU & 10 & FERNANDO MINE \\
\hline CUCU & 15 & FIELD, DELITA \\
\hline CUCU & 17 & FLORIDA DISTRICT \\
\hline CUCU & 20 & GUAIMARO DISTRICT \\
\hline CUCU & 21 & HOLGUIN AREA \\
\hline CUCU & 22 & HOLGUIN DISTRICT \\
\hline CUCU & 5 & ISABEL MARIA \\
\hline $\mathrm{CUCU}$ & 6 & LA MERCEDITA AND LA CONSTANCIA \\
\hline CUCU & 4 & MATAHAMBRE DISTRICT (MONO ET AL) \\
\hline CUCU & 31 & MAYARI DISTRICT (CALEDONIA ET AL) \\
\hline CUCU & 27 & MAYARI-CRISTAL (NICARO) DISTRICT \\
\hline CUCU & 18 & MESA SAN FELIPE \\
\hline
\end{tabular}

AU

N62 03

BA

$U$

$\mathrm{CU}$

ZN PB CU AG CD

$P B$ ZN AG FES

$A G$ PB

NI CU

CU

$\mathrm{CU}$

AU

AG PB ZN

FE TI

AU

CU

$A U$ AG

AU $A \mathrm{~A}$

AU AG

FE

FE

$S$

FE

PB ZN

PB ZN

$\begin{array}{ll}P B & Z N \\ F E & \\ P B & Z N \\ P B & Z N\end{array}$

CR
FES
$A U$

AU

CU

CR
FES
CR

FE

$\mathrm{CU}$

$\mathrm{CU}$

CU PB

FE

FE

AU

CR

BA

CU

CU

FE NI CO

FE
$A U A G$

TI

TI

PB ZN

ZN

PB ZN

PB ZN

CR
W138 50

$\begin{array}{lll}W 133 & 04\end{array}$

$\begin{array}{lll}W 133 & 02 \\ W & 130 & 37\end{array}$

113037

W138 42

W133 23

$\begin{array}{ll}W 128 & 25\end{array}$

W133 12

W135 03

$\begin{array}{lll}W & 32\end{array}$

W135 O3

W136 42

WO84 57

W084 28

WO84 43

W084 05

WOB3 24

WO83 51

W083 39

WO84 36

W084 52

WO83 4

W083 4

WO84 42

W085 43

W083 33

W8 49

W88 18

W084 39

W082 46

W084 09

W084 08

W083 36

W084 27

W084 33

W082 58

W084 18

WO74 38

W079 50

W079 42

W079 42

W083 03"

W077 45

WOB 150

W075 38

W75 38

W084 12

W075 57

W074 48

W076 43

W080 04

W083 01

W078 05

W077 20

W076 18

W076 06

W083 51

W083 43

W083 43

W083 56*

W075 42

WO75 33
4A3B 4 A 7

7C 1C $10 A 2$

BD $1 A 11 A 3$

9A2C7A5

1A 14C5A5

7D 15C3A2

7D 1A11A2+

7DTAT 1A2

4D34C7A5

5B8C5A3

2045B3A5

3A5B TOA

4A7B7A7

4D7C7A7

2A37C4A8

3A 1C7A7

4A37C4A8

उA 1C7A7

$2 A 37 C 4 A 8$

4C7C7A 7

7C7C7A7

3A 1C7A7

4C7C7A 7

2A37C4A8

पA 7 TCAAB

TA56C7A5

11A7C 1OA8

$2 A 37 C 4 A B$

3A47C5A7

7C5C7A7

10B3A9A8

7C7C7A 7

2A7C7A7

7C1C7A7

7C5C7A7

5A58B5A5 -

$11 \mathrm{~B} 45 \mathrm{C}$

$4 A 5 C$

3A5C $11 B 5$

5A58A5A5-

5A58C5A5-

5A58C5A5-

2A45B3A6

3A 1C7A5+

3A15C7A4+

$3 A 5 C 11 A 6$

3D5C $11 A 5$

4A 145C7A4+

$2 A 45 C$

4A45C7A5

4A45C7A5

5A58C5A5-

8D 1C7A4+

3A 1C7A5+

3A1B7B5+

5A58B5A5-

SBSBAA7+ 


\begin{tabular}{|c|c|c|}
\hline $\begin{array}{l}\text { CUCU } \\
\text { CUCU }\end{array}$ & $\begin{array}{l}29 \\
30\end{array}$ & $\begin{array}{l}\text { MOA DISTRICT } \\
\text { MOA-BARACOA DISTRICT (CAYOGUAN ET AL) }\end{array}$ \\
\hline CUCU & 1 & NORTHWEST PINAR DEL RIO BARITE DISTRICT \\
\hline CUCU & 9 & QUEIMADO DISTRICT \\
\hline CUCU & 28 & SAGUA DE TANAMO DISTRICT \\
\hline CUCU & 12 & SANTA CLARA AREA \\
\hline CUCU & 26 & SANTA LUCIA AREA \\
\hline CUCU & 16 & SIGUANEA DISTRICT \\
\hline CUCU & 32 & SOUTH CENTRAL ORIENTE MN DISTRICT \\
\hline CUCU & 35 & SOUTH COAST MN DISTRICT \\
\hline cucu & 25 & SOUTH COAST PROSPECTS \\
\hline CUCU & 23 & SOUTHWEST ORIENTE MN DISTRICT \\
\hline CUCU & 2 & TETE CONTINO (FRANCISCO) MINE \\
\hline DRDR & 2 & FALCONDO (BONAO) \\
\hline DRDR & 6 & LAS MERCEDES (BUCAN POLO) \\
\hline DRDR & 3 & MAIMON-HATILLO \\
\hline DRDR & 1 & MATA GRANDE \\
\hline DRDR & 4 & PUEBLO VIEJO \\
\hline DRDR & 5 & SIERRA DE BAHORUCO (ACEITILLAR) \\
\hline ESES & 2 & EL DORADO \\
\hline ESES & 6 & HORMI GUERO \\
\hline ESES & 4 & LAGUNA DE ALEGRIA \\
\hline ESES & 7 & MONTE MAYOR \\
\hline ESES & 5 & MONTECRISTO AND DIVISADERO \\
\hline ESES & 3 & POTOSI \\
\hline ESES & 1 & SAN JUAN \\
\hline ESES & 8 & SAN SEBASTIAN \\
\hline FRFR & 1 & LANGLADE \\
\hline GLGL & 31 & AMITSOQ \\
\hline GLGL & 12 & ANAP NUNA \\
\hline GLGL. & 25 & ARSUK \\
\hline GLGL & 5 & BLACK ANGEL (MARMORILIK) \\
\hline GLGL & 13 & DISKO BUGT GRAPHITE \\
\hline GLGL & 22 & FISKENAESSET \\
\hline GLGL & 30 & FREDERIK F. MINE \\
\hline GLGL & 26 & GRAENSELAND \\
\hline GLGL & 7 & I GDLUKUNGUAQ \\
\hline GLGL & 28 & ILIMAUSSAQ PROSPECTS (KVANEFJELD, TASEQ) \\
\hline GLGL & 19 & ISUA \\
\hline GLGL & 20 & ITIPILUA \\
\hline GLGL & 24 & IVIGTUT \\
\hline GLGL & 21 & IVISARTOQ \\
\hline GLGL & 27 & JOSVAMINEN \\
\hline GLGL & 11 & KANGATSIAQ \\
\hline GLGL & 10 & KEKERTARSSUATSIAQ \\
\hline GLGL & 29 & KRINGLERNE \\
\hline GLGL & 2 & LANGO \\
\hline GLGL & 14 & LERSLETTEN \\
\hline GLGL & 32 & MALMBJERGET \\
\hline GLGL & 9 & MANERMIUT \\
\hline GLGL & 33 & MESTERSVIG \\
\hline GLGL & 3 & NIAKORNAT \\
\hline GLGL & 15 & NORDRE STROMF JORD \\
\hline GLGL & 1 & NUNATARSSUIT \\
\hline GLGL & 4 & QAERSUT \\
\hline GLGL & 18 & SONDRE ISORTOQ DISTRICT \\
\hline GLGL & 6 & STOROEN \\
\hline GLGL & 16 & SUNGOQ \\
\hline GLGL & 23 & TARTOQ \\
\hline GLGL & 8 & UIVFAQ \\
\hline
\end{tabular}

\begin{tabular}{|c|c|c|c|c|c|c|c|}
\hline \multirow{2}{*}{\multicolumn{3}{|c|}{$\begin{array}{l}\text { FE NI } \quad \mathrm{CO} \\
\mathrm{CR}\end{array}$}} & \multirow{2}{*}{\multicolumn{2}{|c|}{$\begin{array}{ll}\mathrm{N} 20 & 37 \\
\mathrm{~N} 20 & 30\end{array}$}} & wo74 & $\begin{array}{l}57 \\
50 *\end{array}$ & $\begin{array}{l}\text { 5B58A9A7+ } \\
\text { 5A58A5B5 - }\end{array}$ \\
\hline & & & & & W074 & 50* & $\begin{array}{l}\text { 5A58A5B5 - } \\
\text { 8D1C7A4+ }\end{array}$ \\
\hline $\begin{array}{l}\text { BA } \\
\text { MN }\end{array}$ & & & $\begin{array}{l}\text { N22 } \\
\text { N22 }\end{array}$ & $\begin{array}{l}35 \\
50\end{array}$ & $\begin{array}{l}\text { W084 } \\
\text { W080 }\end{array}$ & $\begin{array}{l}02 \\
18\end{array}$ & $\begin{array}{l}\text { 8D 1C7A4+ } \\
\text { 1OA5C9A7+ }\end{array}$ \\
\hline$C R$ & & & $\mathrm{~N} 2 \mathrm{O}$ & 37 & WO75 & 19 & 5A58C5A5 - \\
\hline$A U$ & & & N22 & 20 & W079 & 56 & $4 A C$ \\
\hline$A U$ & & & N21 & 05 & WO75 & 55 & $4 A 5 C$ \\
\hline w & & & N21 & 40 & W082 & 58 & 1A 145C7A4+ \\
\hline MN & & & $\mathrm{N} 2 \mathrm{O}$ & 07 & WO75 & $45 *$ & 10A5B $11 B 6$ \\
\hline MN & & & N19 & 55 & WO77 & $10 *$ & $10 A 5 C 11 C 6$ \\
\hline $\mathrm{CU}$ & & & $\mathrm{N} 2 \mathrm{O}$ & 02 & W076 & $28 *$ & $3 A 5 C+1 B 6$ \\
\hline MN & & & $\mathrm{N} 2 \mathrm{O}$ & 16 & W076 & $27 *$ & 1OA5B $11 B 6$ \\
\hline$C U$ & & & N22 & 23 & WO84 & 07 & 3A1C7A5+ \\
\hline FE & NI CO & & $N 19$ & 01 & W070 & 23 & 5B58A9A7+ \\
\hline AL & & & $N 18$ & 04 & W071 & 38 & 10B 1B9A7+ \\
\hline FE & & & $N 18$ & 58 & W070 & 15 & 2A45C3A5+ \\
\hline $\mathrm{CU}$ & & & N19 & 12 & W071 & 00 & 3A45C5A5+ \\
\hline AU & AG ZN & CU HG & N18 & 57 & W070 & 12 & 4D5B5A5 \\
\hline AL & & & N18 & 08 & W071 & 33 & 10B 1B9A7+ \\
\hline$A U$ & AG & & $N 13$ & 51 & WO88 & 48 & 4C7C7A7 \\
\hline$A U$ & $A G$ & & $N 13$ & 31 & W088 & 03 & 4C7C7A7 \\
\hline $\mathbf{s}$ & & & $N 13$ & 30 & W088 & 30 & 11A7C $10 A 8$ \\
\hline$\Delta U$ & $A G$ & & $\mathrm{~N} 13$ & 42 & W087 & 58 & 4C7C7A7 \\
\hline & AG & & $N 13$ & 36 & WO88 & 04 & 4C7C7A7 \\
\hline & $A G$ & & $N 13$ & 42 & W088 & 20 & 4C7C7A7 \\
\hline PB & $Z N A G$ & & N14 & 17 & WO89 & 22 & 7D14C3A6 \\
\hline$A U$ & & $\mathrm{CU}$ & $N 13$ & 39 & W087 & 55 & 4B785A7 \\
\hline $\mathrm{CU}$ & & & N46 & 54 & WO56 & 15 & $3 A 45 C 7 A 4$ \\
\hline GRF & & & N60 & 18 & WO45 & 08 & $11 E 15 C 1 O A 2$ \\
\hline FE & & & N69 & 58 & W050 & 38 & $2 A 15 C 2 A 2$ \\
\hline GRF & & & N61 1 & 08 & WO48 & 40 & $11 E 15 C 1 O A 2$ \\
\hline $\mathrm{ZN}$ & PB & $\mathbf{A G}$ & N71 & 06 & WO51 & 18 & $7 C 15 B 11 A 2$ \\
\hline GRF & & & N68 & 37 & W052 & 00 & 11E 15C $10 \mathrm{~A} 2$ \\
\hline CR & & $V T I$ & N63 & 06 & Wo5o & 40 & 5A68A8A 1 \\
\hline $\mathrm{cu}$ & & & N60 & 47 & WO45 & 55 & $3 A 15 C 7 A 2$ \\
\hline FE & & & N6 1 & 23 & W047 & 57 & $2 A 5 C 2 A 2$ \\
\hline NI & $\mathrm{CU}$ & & N69 & 52 & W052 & 28 & $5 B 6 C 5 A 7$ \\
\hline$U T$ & H BE NB & & $\mathrm{N} 60$ & 58 & W046 & $\infty$ & 9E2A5A4 \\
\hline & & & N65 & 14 & WO50 & $\infty$ & 2A15A2A1 \\
\hline$C R$ & & & N65 & $\infty$ & W051 & 20 & 5А68B8A 1 \\
\hline CRY & OLITE & & N6 1 & 12 & WO48 & 12 & 1OB2A 1A4 \\
\hline мO & & & N64 & 46 & W050 & 50 & 3B 15C 10A1 \\
\hline cu & & & N60 & 57 & W047 & 55 & 3A 15C7A2 \\
\hline BE & & & N68 & 15 & W053 & 30 & $1 C 4 C 1 A 2$ \\
\hline GRF & & & N68 & 29 & WO53 & 19 & 11E 15C 1042 \\
\hline $\mathbf{Z R}$ & NB & REE & N60 & 52 & WO45 & 50 & $11 \mathrm{C2B8A4}$ \\
\hline GRF & & & N72 & 45 & W056 & 03 & 11E 15C 1OA2 \\
\hline & $\mathrm{ZN}$ & & N68 & 26 & Wo51 & 45 & 3D $15 C_{10 A 2}$ \\
\hline MO & & & N71 & 55 & WO24 & 15 & 3B237B5A8 \\
\hline GRF & & & N68 & 36 & W053 & 11 & $11 E$ 15C $1 O A 2$ \\
\hline PB & $\mathrm{ZN}$ & & N72 & 10 & WO24 & 07 & 7С37B7A8 \\
\hline GRF & & & N7O & 47 & WO53 & 45 & 11E37C 1OA7 \\
\hline GRF & & & N67 & 35 & W053 & 35 & 11E 15C $10 A 2$ \\
\hline GRF & & & N73 & 22 & WO56 & 09 & 11E 15C $1 O A 2$ \\
\hline GRF & & & N70 & 44 & WO52 & 40 & $11 E 37 \mathrm{C} 10 \mathrm{~A} 7$ \\
\hline NI & & & N65 & 26 & WO5 1 & 24 & 5B8B8A 1 \\
\hline FES & & & N70 & 40 & W051 & 24 & 11B5B 10A2 \\
\hline GRF & & & N67 & 10 & W053 & 25 & 1IE 15C 1OA2 \\
\hline $\mathrm{CU}$ & & & N6 1 & 34 & W048 & 30 & 3A 15C 1OA 1 \\
\hline GRF & & & N69 & 25 & W054 & 10 & 11E37C 10A7 \\
\hline
\end{tabular}




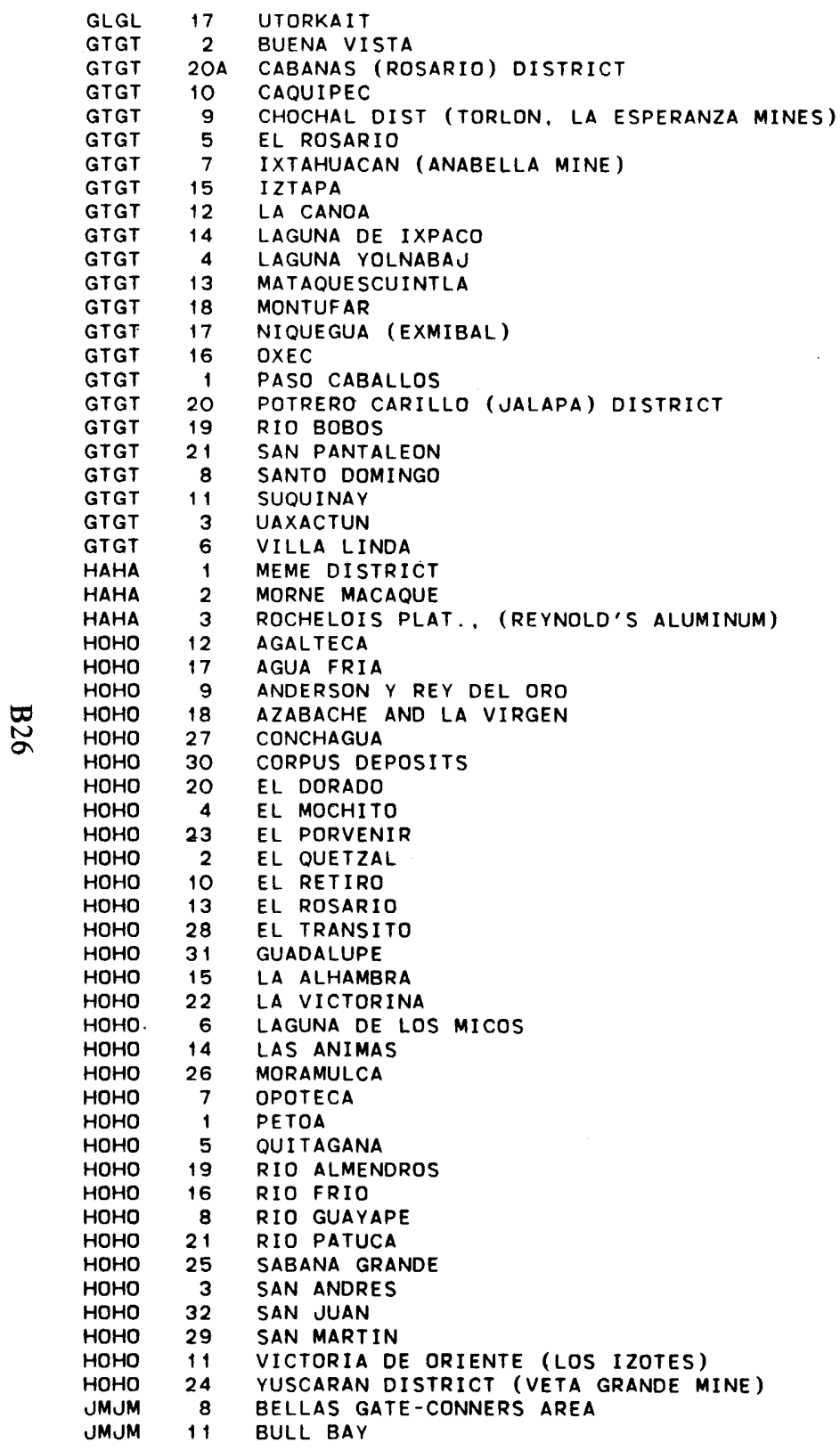

\begin{tabular}{|c|c|c|c|c|c|c|c|}
\hline & N66 & 58 & W053 & 05 & 11E 15C $10 A 2$ \\
\hline \multicolumn{3}{|l|}{ GYP } & N17 & 16 & W090 & 04 & GA 1B6AG \\
\hline \multicolumn{3}{|l|}{ CR } & N14 & 48 & W089 & 47 & 5ABC5A \\
\hline \multirow{2}{*}{\multicolumn{2}{|c|}{ PB ZN }} & CD & N15 & 24 & w090 & 13 & 7C $1 B 7 A G$ \\
\hline \multirow{2}{*}{\multicolumn{2}{|c|}{$\begin{array}{ll}P B & Z N \\
P B & Z N\end{array}$}} & $A G$ & N15 & 24 & WO9 1 & 31 & $7 C 1 C 5 A 6$ \\
\hline & & $\mathbf{A G}$ & N15 & 42 & W09 1 & 37 & 7C 1C5A6 \\
\hline SB & & N15 & 27 & W09 1 & 48 & $8 B 1 C 7 A 6$ \\
\hline \multirow{2}{*}{\multicolumn{3}{|c|}{ TI }} & N13 & 55 & W090 & 39 & $2 A 37 C 4 A 8$ \\
\hline & & & $\mathrm{N}+4$ & 54 & W090 & 27 & $4 A 3 C 4 A 8$ \\
\hline \multirow{2}{*}{\multicolumn{3}{|c|}{5}} & N14 & 13 & w090 & 26 & 11A7C $10 A B$ \\
\hline & & & N16 & 01 & W09 1 & 41 & $7 C 1 C 5 A 6$ \\
\hline \multicolumn{3}{|c|}{$\begin{array}{ll}B & Z N \\
U & A G\end{array}$} & N14 & 30 & w090 & 09 & 3D47C5A7 \\
\hline \multicolumn{2}{|l|}{ NI } & $\mathrm{CO}$ & N15 & 24 & W089 & 06 & 5B8B9A7+ \\
\hline \multicolumn{3}{|r|}{ Co } & N15 & 33 & W089 & 27 & 5B8A9A7+ \\
\hline \multicolumn{3}{|l|}{$\mathrm{CU}$} & N15 & 33 & W089 & 35 & $3 A 456 B 11 A 5$ \\
\hline \multicolumn{3}{|l|}{ GYP } & $\mathrm{N} 17$ & 16 & W090 & 13 & GA 1B6A6 \\
\hline \multicolumn{3}{|l|}{ CR } & N14 & 46 & wo9o & $\infty 0$ & $5 A 8 C 5 A$ \\
\hline$A U$ & & & N15 & 23 & W088 & 43 & $\triangle A 3 C 4 A 8$ \\
\hline $\mathbf{A G}$ & & PB ZN & N14 & 29 & W089 & 24 & 407B7A7 \\
\hline PB & $\mathrm{ZN}$ & $\mathbf{A G}$ & N15 & 26 & W09 1 & 28 & $7 C+C 5 A 6$ \\
\hline PB & $Z N$ & $A G$ & N15 & 14 & W090 & 11 & 7C $1 B 7 A 6$ \\
\hline GYP & & & N17 & 27 & W089 & 42 & 6A 1B6AG \\
\hline PB & $Z \mathrm{~N}$ & $\mathbf{A G}$ & N15 & 40 & W09 1 & 33 & $7 C 1 C 5 A 6$ \\
\hline $\mathrm{cu}$ & & $A U$ & N19 & 38 & W072 & 48 & ЗА45BЗА6 \\
\hline MN & & & $N 19$ & 36 & W072 & 42 & 1OA5C $11 A 6$ \\
\hline AL & & & N18 & 24 & W073 & 12 & IOB 1B9A7+ \\
\hline FE & & & N14 & 27 & W087 & 17 & $2 A 146 C 3 A 6$ \\
\hline$A U$ & $A G C U$ & & N14 & 06 & W086 & 37 & $4 C 14 C 7 A 6$ \\
\hline$A U$ & & & $N 14$ & 33 & w086 & 10 & 4A15C7A3+ \\
\hline$A U$ & AG & & N14 & 06 & W086 & 29 & $4 C 15 C 7 A 5+$ \\
\hline$\Delta U$ & & & $\mathrm{~N} 13$ & 48 & W086 & 28 & 4A 15C7A6 \\
\hline AU & & & $N 13$ & 16 & W087 & 01 & 4A7C7A7 \\
\hline$A U$ & & & N15 & 34 & W085 & 16 & $4 A 3 C 4 A 8$ \\
\hline$\Delta G$ & PB ZN & $C D A U$ & N14 & 51 & W088 & 04 & 7D $1 B 5 A 6$ \\
\hline AU & $A G$ & & N13 & 46 & W087 & 42 & 4C7C7A7 \\
\hline SB & & & N14 & 47 & W088 & 58 & $8 B 15 C 7 A 6$ \\
\hline$A U$ & & & N14 & 34 & W086 & 19 & $\triangle A 3 C 4 A B$ \\
\hline AG & AU & & $N 14$ & 15 & W087 & 04 & $4 C 14 B 7 A 6$ \\
\hline$A U$ & AG & & $N 13$ & 30 & W087 & 36 & 4C7C7A7 \\
\hline$A U$ & & & $\mathrm{~N} 13$ & 19 & W086 & 52 & 4A7C7A 7 \\
\hline AU & AG & & N14 & 15 & W086 & 16 & $4 C 15 C 7 A 3+$ \\
\hline$A U$ & AG & & $\mathrm{N} 13$ & 52 & W087 & 33 & 4C7C7A7 \\
\hline FE & & TI & N15 & 48 & W087 & 37 & $2 A 37 C 4 A B$ \\
\hline $\mathbf{A G}$ & PB ZN & & N14 & 09 & W087 & 02 & 4D 1B5A6 \\
\hline$A U$ & $A G$ & & N13 & 41 & W087 & 18 & 4C7C7A7 \\
\hline $\mathbf{A G}$ & PB ZN & & N14 & 35 & W087 & 44 & $4 D 1 C 7 A 6$ \\
\hline $\mathrm{CU}$ & & & N15 & 16 & W088 & 19 & 3A 146C3A6 \\
\hline $\mathrm{ZN}$ & CU $\mathbf{A G}$ & & N14 & 37 & W088 & 23 & $301 C 7 A 6$ \\
\hline$A U$ & & & N14 & 04 & W086 & 23 & $4 A 3 C 4 A 8$ \\
\hline$A U$ & & & N14 & 14 & W086 & 07 & $\triangle A 3 C 4 A B$ \\
\hline$\Delta U$ & & & $\mathrm{~N} 14$ & 45 & W086 & 02 & AA3C $4 A B$ \\
\hline$A U$ & & & $N+4$ & 33 & W085 & 25 & $\triangle A 3 C 4 A B$ \\
\hline AU & $A G$ & & $\mathrm{~N} 13$ & 48 & W087 & 15 & 4C7C7A7 \\
\hline$A U$ & & & N14 & 45 & W088 & 56 & 4A7C7A7 \\
\hline$A U$ & & & $N 13$ & 12 & W087 & $\infty$ & 4A7C7A7 \\
\hline$A U$ & AG & & $N 13$ & 25 & W087 & 16 & 4C7C7A7 \\
\hline HG & & & N14 & 29 & W087 & 12 & 8A TC7A6+ \\
\hline$A U$ & AG & & $N+3$ & 55 & WO86 & 51 & 4C7C7A7 \\
\hline $\mathrm{CU}$ & & $A U A G$ & $N 18$ & 10 & W077 & 11 & 3A45B5A5+ \\
\hline GYP & & & N17 & 59 & W076 & 39 & 6A5B6A6 \\
\hline
\end{tabular}




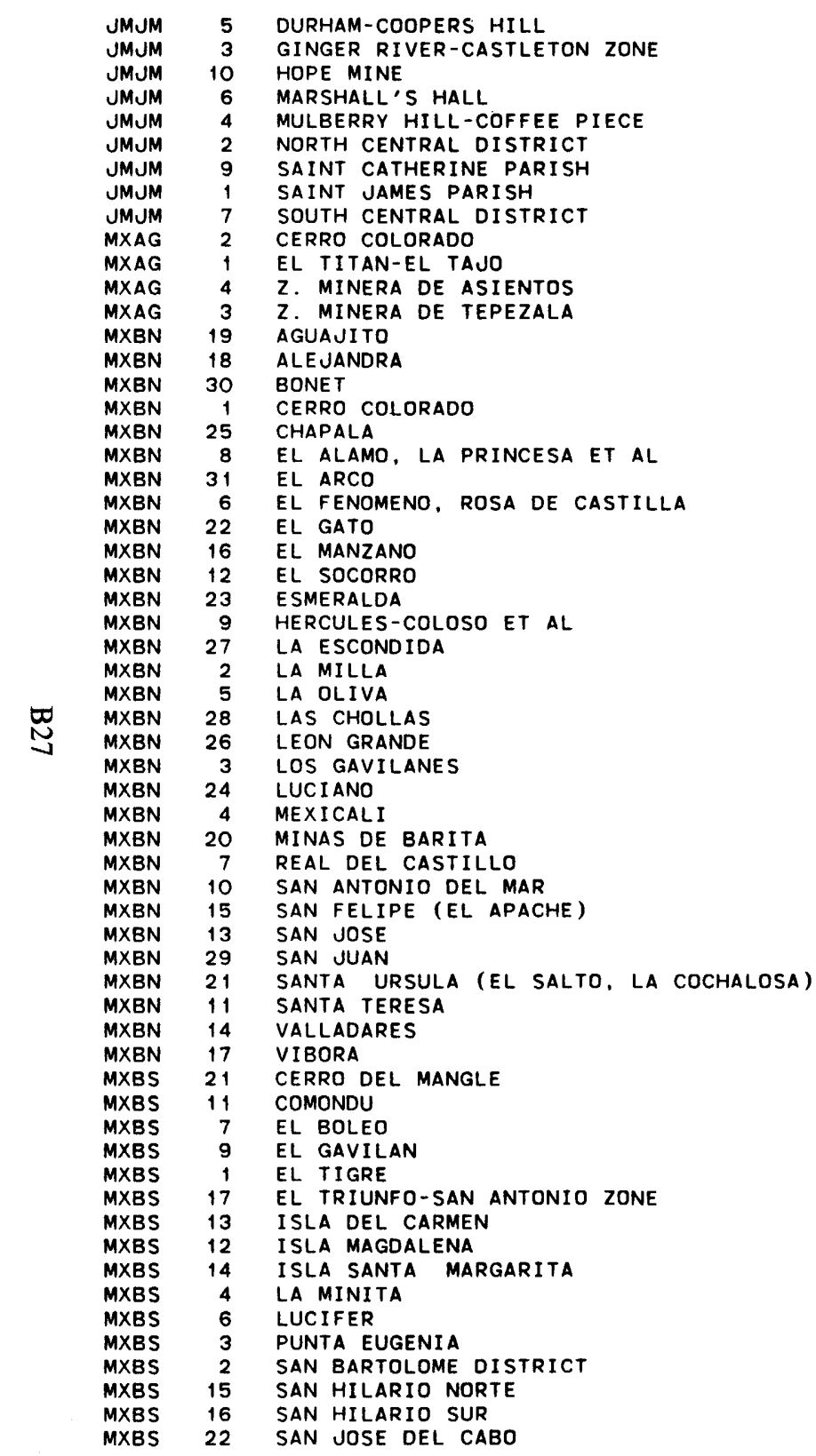

\begin{tabular}{|c|c|c|c|c|c|c|c|}
\hline $\mathrm{cu}$ & & & N18 & 12 & WO76 & 30 & 3A45C7A \\
\hline cu & & & $N+8$ & 14 & W076 & 49 & $3 A 5 C$ \\
\hline $\mathrm{ZN}$ & PB & $A G A U C U$ & N18 & 07 & W076 & 44 & $7 D 5 C 11 A 6$ \\
\hline MN & & & N18 & 08 & W076 & 26 & $10 \mathrm{~A} 5 \mathrm{C}$ \\
\hline FE & & & N18 & 12 & w076 & 35 & 2A45C7A5+ \\
\hline$A L$ & & & N18 & 21 & W077 & $17 *$ & 1OB 1A9B7+ \\
\hline AL & & & $N 18$ & 02 & W077 & 03 & IOB 1B9A7+ \\
\hline$A L$ & & & N18 & 20 & W077 & 54 & 1OB 1B9A7+ \\
\hline$A L$ & & & N18 & 02 & W077 & $28 *$ & 1OB 1A9B7+ \\
\hline SN & & & N22 & 20 & $W 102$ & 19 & $1 B 7 C 7 A 7$ \\
\hline SN & & & N22 & 22 & $W 102$ & 27 & 1B7C7A7 \\
\hline AU & $\mathrm{CU}$ & & N22 & 15 & $W 102$ & 05 & $4 B 15 C 7 A 7$ \\
\hline$A U$ & $\mathrm{CU}$ & & N22 & 14 & $W 102$ & 10 & AB 15C7A7 \\
\hline $\mathrm{cu}$ & & & N3O & 03 & $W 115$ & 28 & 3A $145 C 7 A 6$ \\
\hline $\mathrm{cu}$ & & & N3O & 10 & $w+15$ & 22 & 3A4C7A7 \\
\hline $\mathrm{cu}$ & FE & $A U A G$ & N28 & 20 & $w 113$ & 54 & 3A 15C7A5 \\
\hline $\mathrm{cu}$ & & & N32 & 28 & $W 116$ & 55 & 3A147C7A5 \\
\hline $\mathrm{cu}$ & & & N29 & 40 & $W 114$ & 25 & 3A4C $3 A 5$ \\
\hline AU & $A G$ & & N31 & 40 & $W 116$ & 05 & 4C4C7A5 \\
\hline $\mathrm{cu}$ & & AU MO & N28 & 10 & W113 & 15 & 3А45A5A5 \\
\hline $\mathbf{w}$ & & & N31 & 58 & $W+16$ & $\infty$ & 1A14B3A5 \\
\hline $\mathrm{cu}$ & & FE & N29 & 53 & $W 115$ & 17 & 3A14C3A6 \\
\hline$F E$ & & & N3O & 17 & $W 115$ & 37 & $2 A 5 C 7 A 5$ \\
\hline$A U$ & & & N31 & 02 & W115 & 45 & 4A 14C3A5 \\
\hline $\mathrm{cu}$ & & & N29 & 45 & $W 115$ & 10 & 3A $1 C 5 A 6$ \\
\hline $\mathrm{FE}$ & & & N31 & 17 & $W 116$ & 15 & $2 A 14 C 3 A 5$ \\
\hline$A U$ & & & N29 & 05 & $W 113$ & 40 & $4 A+5 C 7 A 7$ \\
\hline $\mathbf{A U}$ & & & N32 & 15 & $w 116$ & 00 & 4A4C7A5+ \\
\hline $\mathbf{w}$ & & & N32 & 05 & $W 115$ & 45 & 1A14C3A5 \\
\hline$\Delta U$ & & & N28 & 50 & $W 113$ & 30 & 4A 15C7A5+ \\
\hline$A U$ & & & N29 & 15 & $W 114$ & 25 & AA $15 C 7 A 6$ \\
\hline$w$ & & & N32 & 10 & $W 116$ & 10 & TA14C3A5 \\
\hline $\mathrm{cu}$ & & & N29 & 40 & $w+15$ & 05 & 3A $1 C 5 A G$ \\
\hline TLC & & & N32 & 35 & $W \$ 15$ & 30 & 10E BC5A \\
\hline BA & & & N3O & oo & $W 114$ & 40 & 8D4C7A7 \\
\hline$A U$ & & & N31 & 55 & $W 116$ & 15 & 4A 14C7A5 \\
\hline AU & $\mathrm{CU}$ & FE & N31 & 05 & $W 116$ & 15 & 4B 4C7A5 \\
\hline $\mathbf{s}$ & & & N31 & $\infty$ & $W 114$ & 50 & $11 A C 7 A 7$ \\
\hline $\mathrm{cu}$ & & & N31 & 05 & $W 115$ & 35 & 3A4C7A5+ \\
\hline$A U$ & & & N28 & 40 & $W 113$ & 30 & 4A 15C7A5+ \\
\hline FE & & & N29 & 55 & $W 1+5$ & 25 & 2A 14B5A5 \\
\hline$A U$ & $\mathrm{CU}$ & FE & N31 & 05 & $W 116$ & 01 & 4B $147 C 7 A 5$ \\
\hline AU & & & N31 & 01 & $W 115$ & 25 & 4A AC7A \\
\hline $\mathrm{cu}$ & & & N30 & 10 & W115 & 46 & 3A4C7A \\
\hline CU & & & N23 & 25 & $W 109$ & 32 & 3AC 7A7 \\
\hline TLC & & & N26 & 05 & $W 111$ & 50 & 10E 8C5A \\
\hline $\mathrm{cu}$ & ZN & $\mathbf{A G}$ & N27 & 17 & $W 1+2$ & 18 & 3D 17A 1OA7 \\
\hline MN & & & N26 & 52 & $W 111$ & 48 & 10A7C7A7 \\
\hline CR & & & N27 & 33 & $W 114$ & 42 & 5A58C5A4 \\
\hline & $Z N A G A U$ & & N23 & 47 & $W 110$ & 03 & 4DB7A7 \\
\hline NA & & & N24 & 54 & W111 & 11 & $6 B 37 \mathrm{C} 6 \mathrm{~A}$ \\
\hline MG & & & N24 & 54 & $w 112$ & 13 & 6D58C5A4 \\
\hline MG & & & N24 & 30 & $W 111$ & 55 & $6058 C 5 A 4$ \\
\hline$A U$ & & & N27 & 29 & $W 114$ & 11 & 4A 15C7A \\
\hline MN & & & N27 & 22 & $W 1+2$ & 23 & 1OA $17 B 11 A 7$ \\
\hline ASB & & & N27 & 27 & $W 114$ & 15 & SE58C5A4 \\
\hline MG & & & N27 & 28 & $W 114$ & 25 & 6058C5A4 \\
\hline $\mathbf{p}$ & & & N24 & 28 & $w 111$ & 09 & 2E 37B2B 7 \\
\hline $\mathrm{P}$ & & & N24 & 17 & $w 111$ & oo & 2E37B2B7 \\
\hline AU & AG & $w$ & N23 & 04 & W 109 & 40 & $4 C 4 C 7 A 7$ \\
\hline
\end{tabular}




\begin{tabular}{|c|c|c|}
\hline MXBS & 8 & SAN MARCOS \\
\hline MXBS & 5 & SAN ROQUE \\
\hline MXBS & 10 & SANTA ISABEL (SAN NICOLAS) \\
\hline MXBS & 20 & SANT I AGO \\
\hline MXBS & 18 & TODOS SANTOS \\
\hline MXBS & 19 & TODOS SANTOS \\
\hline $\mathrm{MXCH}$ & 74 & ADELA \\
\hline $\mathrm{MXCH}$ & 134 & AGUA CALIENTE \\
\hline $\mathrm{M} \times \mathrm{CH}$ & 64 & AGUA NUEVA \\
\hline $\mathrm{MXCH}$ & 66 & AGUA NUEVA \\
\hline $\mathrm{MXCH}$ & 82 & AGUAJE \\
\hline $\mathrm{MXCH}$ & 104 & ALDAMA \\
\hline $\mathrm{MXCH}$ & 163 & ALISOS \\
\hline $\mathrm{MXCH}$ & 77 & ALKADEFF \\
\hline $\mathrm{MXCH}$ & 35 & APACHE \\
\hline $\mathrm{MXCH}$ & 16 & ASCENCION AREA \\
\hline $\mathrm{MXCH}$ & 141 & BARRANCA DEL COBRE \\
\hline $\mathrm{MXCH}$ & 149 & BATOPILAS \\
\hline $\mathrm{MXCH}$ & 122 & BATOPILILLAS \\
\hline $\mathrm{MXCH}$ & 128 & BATOSEGACHI, EL POTRERO \\
\hline $\mathrm{MXCH}$ & 12 & BI SMARK \\
\hline $\mathrm{MXCH}$ & 42 & BONANZA \\
\hline $\mathrm{MXCH}$ & 96 & BOQUILLITA \\
\hline $\mathrm{MXCH}$ & 81 & BUENOS AIRES \\
\hline $\mathrm{MXCH}$ & 158 & CALABACILLAS \\
\hline $\mathrm{MXCH}$ & 76 & CALERA \\
\hline $\mathrm{MXCH}$ & 169 & CAMARGO \\
\hline $\mathrm{MXCH}$ & 132 & CARICHIC \\
\hline $\mathrm{MXCH}$ & 102 & CARR I ZALILLO \\
\hline $\mathrm{MXCH}$ & 2 & CASA DE JANOS \\
\hline $\mathrm{MXCH}$ & 30 & CASAS GRANDES AREA \\
\hline $\mathrm{MXCH}$ & 161 & CEBOLLITAS, LOS ANGELES \\
\hline $\mathrm{M} \times \mathrm{CH}$ & 184 & CENICEROS DE ABAJO \\
\hline $\mathrm{MXCH}$ & 165 & CENIZA \\
\hline $\mathrm{MXCH}$ & 50 & CERRO BOLUDO, PARAGATOS \\
\hline $\mathrm{MXCH}$ & 178 & CERRO DE SANTA ANA \\
\hline $\mathrm{MXCH}$ & 21 & CERRO DEL CHILE \\
\hline $\mathrm{MXCH}$ & 145 & CERRO DEL COMAL, NAVEGANTE \\
\hline $\mathrm{MXCH}$ & 22 & CERRO DEL PLOMO \\
\hline $\mathrm{MXCH}$ & 103 & CERRO PRIETO \\
\hline $\mathrm{MXCH}$ & 176 & CERROS COLORADOS \\
\hline $\mathrm{MXCH}$ & 127 & CHINIPAS \\
\hline $\mathrm{M} \times \mathrm{CH}$ & 135 & CIENEGUITA \\
\hline $\mathrm{MXCH}$ & 151 & COLORES \\
\hline $\mathrm{MXCH}$ & 52 & CONCAMENA-YOQUIVO-COJUR I CHIC \\
\hline $\mathrm{MXCH}$ & 49 & CONCHENITO \\
\hline $\mathrm{MXCH}$ & 11 & CONEJOS \\
\hline $\mathrm{MXCH}$ & 185 & CORONADO \\
\hline $\mathrm{MXCH}$ & 92 & COYAME \\
\hline $\mathrm{MXCH}$ & 20 & CUATROS AMIGOS \\
\hline $\mathrm{MXCH}$ & 95 & CUCHILLO PARADO \\
\hline $\mathrm{MXCH}$ & 80 & CUSIHUIRIACHIC \\
\hline $\mathrm{M} \times \mathrm{CH}$ & 189 & DOLORES \\
\hline $\mathrm{MXCH}$ & 48 & DOLORES, HUISOPA \\
\hline $\mathrm{MXCH}$ & 41 & EL ALAMILLO \\
\hline $\mathrm{M} \times \mathrm{CH}$ & 54 & EL CABIRO \\
\hline $\mathrm{M} \times \mathrm{CH}$ & 37 & EL CARRIZO \\
\hline $\mathrm{MXCH}$ & 110 & EL COYOTE \\
\hline $\mathrm{M} \times \mathrm{CH}$ & 51 & EL MADRONO \\
\hline $\mathrm{MXCH}$ & 7 & EL MIMBRE \\
\hline
\end{tabular}

\begin{tabular}{|c|c|c|c|c|c|c|c|c|}
\hline & N27 & 13 & $w 112$ & 05 & GA37A6A7 \\
\hline \multicolumn{4}{|c|}{$\begin{array}{l}\text { GYP } \\
\text { CU }\end{array}$} & N27 & 15 & W114 & 24 & $3 A 5 C 7 A$ \\
\hline \multicolumn{4}{|l|}{ MN } & N26 & 29 & $w 111$ & 33 & 10A7C7A7 \\
\hline \multirow{2}{*}{\multicolumn{4}{|c|}{$A \cup A G$}} & N23 & 30 & $W 109$ & 45 & 4CC7A7 \\
\hline & & & & N23 & 30 & $w+10$ & 12 & $3 A C 7 A 7$ \\
\hline \multirow{2}{*}{\multicolumn{4}{|c|}{$\begin{array}{l}A U A G \\
M N\end{array}$}} & N23 & 26 & W110 & 11 & 4CC7A7 \\
\hline & & & & N28 & 41 & W106 & 40 & 10A7C7A7 \\
\hline \multicolumn{4}{|l|}{ MN } & N27 & 52 & W106 & 11 & $10 A 7 C 7 A 7$ \\
\hline \multicolumn{4}{|l|}{$\mathbf{F}$} & N29 & 52 & W106 & 22 & $8 C 14 C+\backslash A 7$ \\
\hline \multicolumn{4}{|l|}{ MN } & N29 & 41 & W 106 & 12 & 10A7C7A7 \\
\hline \multirow{2}{*}{\multicolumn{4}{|c|}{$\begin{array}{l}\text { FE } \\
C U\end{array}$}} & N28 & 18 & $w 106$ & 29 & $2 A 14 C 3 A 7$ \\
\hline $\mathrm{cU}$ & & & & N28 & 53 & W105 & 54 & 3A $1 C 7 A 7$ \\
\hline \multirow{2}{*}{\multicolumn{4}{|c|}{$\begin{array}{l}\text { MO } \\
\text { MN }\end{array}$}} & N26 & 11 & W107 & 19 & 3B 7C7A7 \\
\hline & & & & N28 & 36 & W107 & 40 & 10A7C7A7 \\
\hline \multicolumn{4}{|l|}{ MN } & N30 & 20 & W107 & 32 & 10A7C7A 7 \\
\hline \multicolumn{4}{|l|}{ MN } & N30 & 55 & $W 107$ & 30 & 10A7C7A7 \\
\hline$A G$ & PB ZI & ZN & CU AU & N27 & 29 & W107 & 35 & 407C5A7 \\
\hline$A U$ & \multicolumn{3}{|c|}{$A G$} & N27 & 02 & W 107 & 44 & 4C47B7A7 \\
\hline HG & & & & N27 & 53 & $W 108$ & 26 & BA7C7A7 \\
\hline$A U$ & AG & & & N27 & 23 & $W 108$ & 20 & 4C7C7A7 \\
\hline$A U$ & $A G C$ & $\mathrm{CU}$ & & N31 & 14 & W107 & 35 & 4C7C7A7 \\
\hline PB & $A G$ & & & N30 & 51 & W105 & 39 & $701 C 7 A 7$ \\
\hline PB & ZN A & $\Delta G$ & & N29 & 16 & $W 104$ & 46 & $7 D+C 7 A 7$ \\
\hline$A G$ & & & & N28 & 14 & $W 106$ & 45 & 4D7C7A7 \\
\hline$A U$ & AG & & & N26 & 20 & $W 107$ & 41 & 4C7C7A7 \\
\hline $\mathrm{ZN}$ & PB A & $A G$ & & N28 & 38 & W 107 & 25 & 7D 1C7A7 \\
\hline GYP & & & & N27 & 41 & W105 & 14 & 6A1C6A5 \\
\hline$S B$ & & & & N27 & 58 & W106 & 59 & $8 B 7 C 7 A 7$ \\
\hline FE & & & & N28 & 57 & W105 & 12 & 2A14C3A7 \\
\hline MN & & & & N3O & 43 & $W 108$ & 31 & 10A7B 7A7 \\
\hline MN & & & & N3O & 29 & $W 107$ & 59 & 10A7B7A7 \\
\hline$A U$ & $A G$ & & & N26 & 21 & W107 & 15 & 4C7C7A7 \\
\hline MN & & & & N26 & 48 & $W 105$ & 21 & $10 A 7 C 7 A 7$ \\
\hline$A U$ & $\mathbf{A G}$ & & & N26 & 10 & W106 & 31 & 4C7C7A7 \\
\hline$A U$ & $\mathbf{A G}$ & & & N28 & 21 & W108 & 32 & 4C7B7A7 \\
\hline$w$ & & & & N27 & 03 & W105 & 08 & \{ATC7AT \\
\hline PB & $\Delta G$ & & & N30 & 36 & W 107 & 04 & 707C7A7 \\
\hline$A U$ & $A G$ & & & N27 & 02 & W106 & 31 & 4C7C7A7 \\
\hline PB & $\mathbf{A G}$ & & & N3O & 27 & $W 106$ & 55 & 7D7C7A7 \\
\hline PB & $A G$ & & & N28 & 53 & $W 105$ & 23 & $7 D 157 C 5 A 7$ \\
\hline$H G$ & & & & N26 & 57 & $W 104$ & 36 & 8A7C7A7 \\
\hline$A U$ & $\mathbf{A G}$ & & & N27 & 26 & $W 108$ & 31 & 4C7B7A7 \\
\hline $\mathrm{ZN}$ & PB & & & N27 & 50 & $W 106$ & 00 & $7 C 1 C 7 A 7$ \\
\hline$A U$ & $A G$ & & & N26 & 53 & W107 & 41 & 4C7C7A7 \\
\hline$A G$ & PB Z & ZN & $\mathrm{AU} C \mathrm{CU}$ & N28 & 17 & W108 & 08 & 4D7C7A7 \\
\hline$A U$ & $A G$ & & & N28 & 32 & $W 108$ & 18 & 4C7C7A7 \\
\hline$A G$ & $A U$ & & & N31 & 17 & $W 107$ & 11 & 4C7C7A 7 \\
\hline MN & & & AU & N26 & 44 & $W 105$ & 05 & 10A7C7A7 \\
\hline $\mathrm{CU}$ & & & & N29 & 29 & $W 105$ & 05 & 3A 1C5A7 \\
\hline MN & & & & N30 & 35 & $W 107$ & 32 & 10A7C7A7 \\
\hline NA & & & & N29 & 20 & W104 & 55 & 6B $1 C 6 A 8$ \\
\hline$A G$ & PB Z & $\mathrm{ZN} \mathrm{CU}$ & & N28 & 15 & W106 & 52 & 407C7A7 \\
\hline$A U$ & $A G$ & & & N25 & 57 & $W 107$ & 07 & 4C7C7A7 \\
\hline$A U$ & $A G$ & & & N28 & 59 & W108 & 34 & 4C7C7A7 \\
\hline PB & $A G$ & & & N3O & 56 & W105 & 46 & 7D1C7A7 \\
\hline$A U$ & $A G$ & & & N28 & 13 & $W 108$ & 30 & 4C7C7A7 \\
\hline MN & & & & N30 & 13 & $W 106$ & 40 & 10A7C7A7 \\
\hline $\mathrm{FE}$ & cu & & & N28 & 38 & $W 104$ & 05 & 2D7C7A7 \\
\hline$A U$ & $A G$ & & & N28 & 20 & W 108 & 20 & 4C7C7A7 \\
\hline$A U$ & $A G$ & & & N31 & 20 & $W 106$ & 04 & 4C 1C7A7 \\
\hline
\end{tabular}




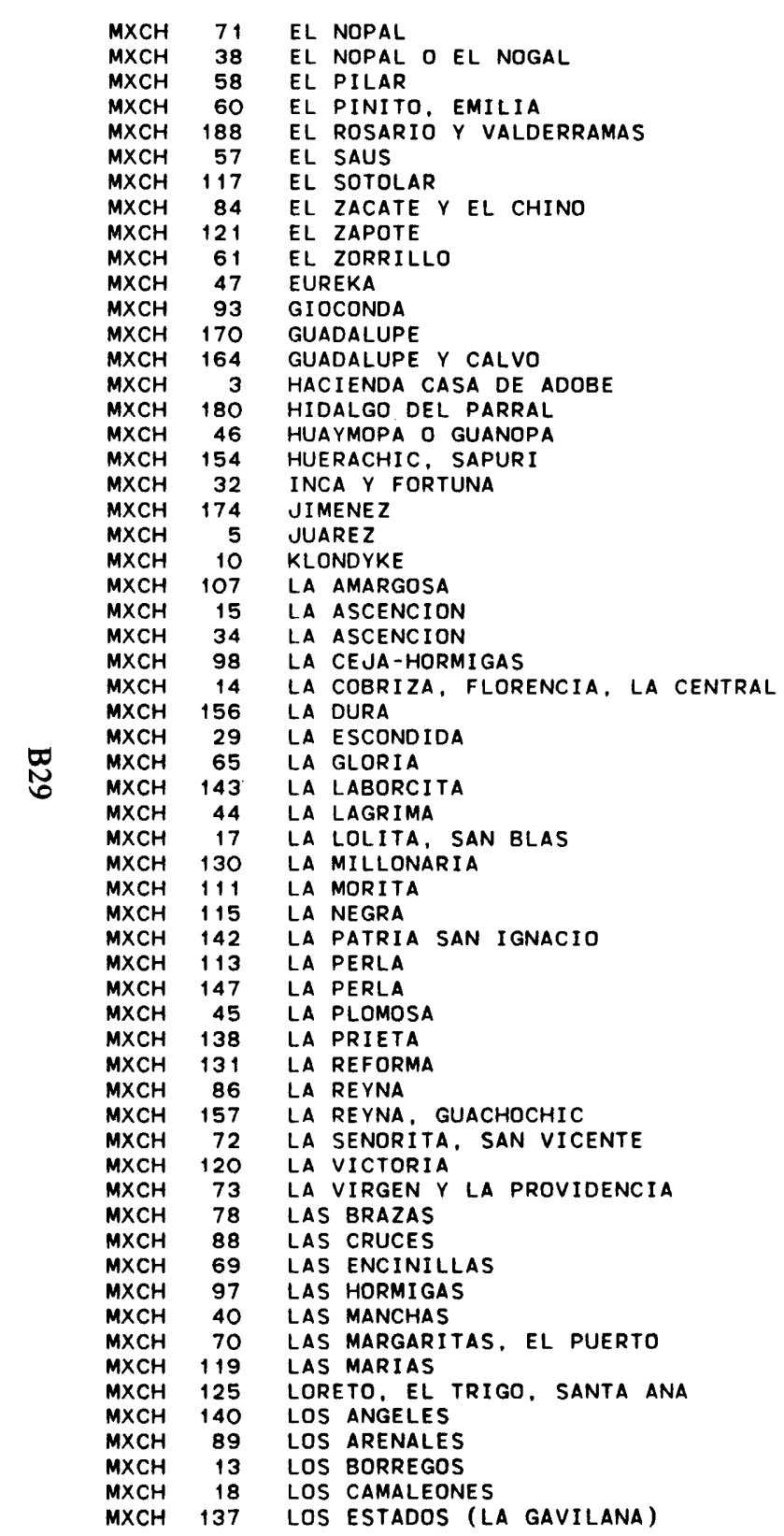

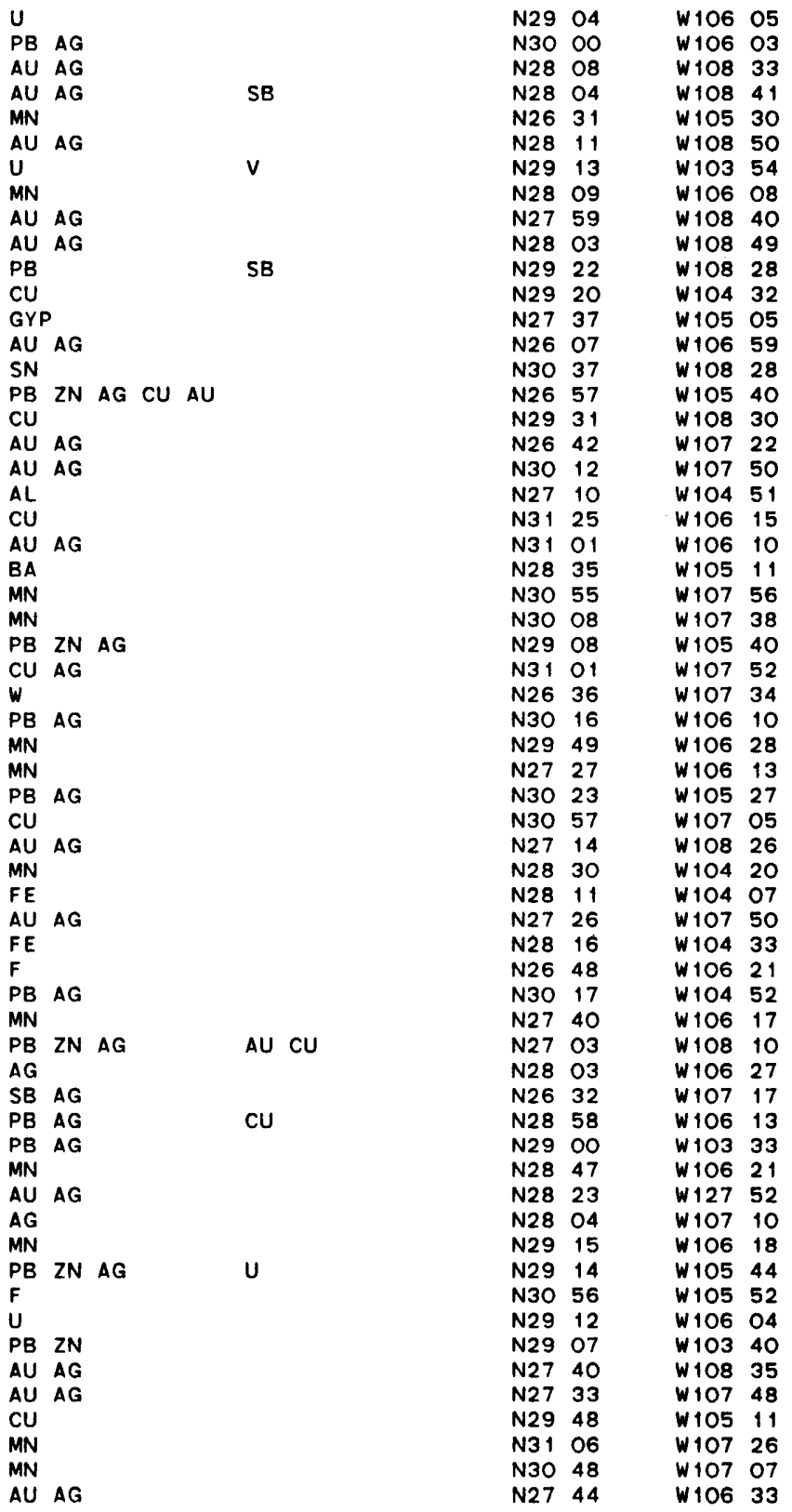

9A7C7A7 707C7A7

4C 14C3A7 4C15C7A7 1OA7C7A7 QE 1C7A7 9E 1C7A7 10A7C7A7 4C 17C3A7 4C7C7A7 7A 1C7A7 3A1C7A7 6A1C6A5 4C7A7A7 1B7C7A7 7D7A7A7 3A7C7A7 $\triangle C 7 C 7 A 7$ 4C7C7A7 1OB 1B5A7 3A5C 10A4 3A5C 1OA 4
4C 1C7A 7 4C1C7A7 8D 1C1OA4 1OA7C7A7 10A7C7A7 7D17C7A7 3D7C7A7 1A7C7A7 407C7A7 10A7C7A7 10A7C7A7 7D 1C7A7 3A $14 C 3 A 7$ 4C7C7A7 10A7C7A7 2A7C5A7 4C7C7A7 2A7B5A7 8C147C7A7 7D1C7A7 10A7C7A7 707A5A7 407C7A7 8B7C7A7 7D7C7A7 701C7A7 10A7C7A7 4C7C7A7 4D7C7A7 10A7C7A7 7D 1C5A7 $8 C 14 C 11 A 7$ 9A7C7A7 7C1C5A7 4C7C7A7 3A1C5A7 10A7C7A7 10A7C7A 4C7C7A7 


\begin{tabular}{|c|c|c|}
\hline $\mathrm{MXCH}$ & 175 & LOS REYES \\
\hline $\mathrm{M} \times \mathrm{CH}$ & 101 & LOS VOLCANES \\
\hline $\mathrm{MXCH}$ & 136 & MAGUARICHIC, SANTA MARTA \\
\hline $\mathrm{MXCH}$ & 87 & MILPILLAS \\
\hline $\mathrm{MXCH}$ & 173 & MINAS NUEVAS \\
\hline $\mathrm{M} \times \mathrm{CH}$ & 179 & MINAS NUEVAS \\
\hline $\mathrm{M} \times \mathrm{CH}$ & 75 & MINILLAS Y OTRAS \\
\hline $\mathrm{MXCH}$ & 153 & MOL INARES \\
\hline $\mathrm{MXCH}$ & 126 & MONTERDE \\
\hline $\mathrm{MXCH}$ & 68 & NAMIQUIPA LA VENTUROSA \\
\hline $\mathrm{M} \times \mathrm{CH}$ & 139 & NONOAVA \\
\hline $\mathrm{M} \times \mathrm{CH}$ & 31 & NUEVO CASAS GRANDES \\
\hline $\mathrm{MXCH}$ & 63 & OJO CALIENTE \\
\hline $\mathrm{M} \times \mathrm{CH}$ & 186 & PAUARITO \\
\hline $\mathrm{MXCH}$ & 27 & PALO BLANCO (LAMENTOS B) \\
\hline $\mathrm{M} \times \mathrm{CH}$ & 85 & PALOMAS \\
\hline $\mathrm{MXCH}$ & 79 & PEDERNALES \\
\hline $\mathrm{MXCH}$ & 187 & PIEDRAS BLANCAS \\
\hline $\mathrm{MXCH}$ & 177 & PILONCILLOS \\
\hline $\mathrm{MXCH}$ & 99 & PLOMOSAS \\
\hline $\mathrm{MXCH}$ & 155 & POTRERO DE BOJORQUES, LA GPNA. \\
\hline $\mathrm{M} \times \mathrm{CH}$ & 191 & RAFAEL, POTRERO DE ORPINEDA, CUERVO \\
\hline $\mathrm{MXCH}$ & 1 & RANCHO DEL MEDIO \\
\hline $\mathrm{MXCH}$ & 106 & ROQUE \\
\hline $\mathrm{M} \times \mathrm{CH}$ & 116 & SABONAROLA \\
\hline $\mathrm{MXCH}$ & 6 & SAMALAYUCA \\
\hline $\mathrm{M} \times \mathrm{CH}$ & 56 & SAN ANTONIO \\
\hline $\mathrm{MXCH}$ & 112 & SAN BERNARDINO \\
\hline $\mathrm{MXCH}$ & 118 & SAN CARLOS \\
\hline $\mathrm{MXCH}$ & 182 & SAN FRANCISCO DEL ORO \\
\hline $\mathrm{MXCH}$ & 9 & SAN IGNACIO \\
\hline $\mathrm{M} \times \mathrm{CH}$ & 150 & SAN IGNACIO \\
\hline $\mathrm{MXCH}$ & 83 & SAN ISIDRO \\
\hline $\mathrm{M} \times \mathrm{CH}$ & 33 & SAN JOAQUIN \\
\hline $\mathrm{M} \times \mathrm{CH}$ & 152 & SAN JOAQUIN, MORELOS \\
\hline $\mathrm{MXCH}$ & 160 & SAN JOSE DE LAS CRUCES \\
\hline $\mathrm{MXCH}$ & 39 & SAN JUAN \\
\hline $\mathrm{MXCH}$ & 171 & SAN JUAN CORDERO \\
\hline $\mathrm{MXCH}$ & 159 & SAN JUAN NEPOMUCENO \\
\hline $\mathrm{MXCH}$ & 166 & SAN JULIAN \\
\hline $\mathrm{MXCH}$ & 167 & SAN JULIAN \\
\hline $\mathrm{M} \times \mathrm{CH}$ & 124 & SAN LUIS \\
\hline $\mathrm{MXCH}$ & 109 & SAN MIGUEL \\
\hline $\mathrm{MXCH}$ & 19 & SAN PEDRO CORRALITOS \\
\hline $\mathrm{MXCH}$ & 53 & SANTA MARGARITA, MARIA VIRGINIA \\
\hline $\mathrm{MXCH}$ & 55 & SANTA MARIA, SAHUAYACANCITO \\
\hline $\mathrm{M} \times \mathrm{CH}$ & 183 & SANTA BARBARA \\
\hline $\mathrm{MXCH}$ & 105 & SANTA EULALIA \\
\hline $\mathrm{M} \times \mathrm{CH}$ & 162 & SANTA RITA \\
\hline $\mathrm{M} \times \mathrm{CH}$ & 108 & SANTA TERESA \\
\hline $\mathrm{M} \times \mathrm{CH}$ & 133 & SATEVO \\
\hline $\mathrm{MXCH}$ & 168 & SAUCILLO-NAICA \\
\hline $\mathrm{MXCH}$ & 100 & SIERRA CHILICOTE \\
\hline $\mathrm{M} \times \mathrm{CH}$ & 114 & SIERRA DE ENCINILLAS \\
\hline $\mathrm{M} \times \mathrm{CH}$ & 8 & SIERRA DE GUADALUPE \\
\hline $\mathrm{M} \times \mathrm{CH}$ & 26 & SIERRA DE LA ALCAPARRA \\
\hline $\mathrm{M} \times \mathrm{CH}$ & 25 & SIERRA DE LA MAGDALENA \\
\hline $\mathrm{M} \times \mathrm{CH}$ & 91 & SIERRA DE LAS DAMAS \\
\hline $\mathrm{M} \times \mathrm{CH}$ & 28 & SIERRA DE LAS MINAS \\
\hline $\mathrm{M} \times \mathrm{CH}$ & 24 & SIERRA DE LOS MOSOUETEROS \\
\hline
\end{tabular}

\begin{tabular}{|c|c|c|c|c|c|c|c|c|c|c|}
\hline $\mathrm{CU}$ & & & & $w$ & BA & N27 & 02 & $W 104$ & 51 & $3 A 14 C 3 A 7$ \\
\hline MN & & & & & & N28 & 59 & W 104 & 09 & 10A7C7A7 \\
\hline$A U$ & $A G$ & & & & & N27 & 51 & W 107 & 59 & 4C7C7A7 \\
\hline PB & $\mathbf{A G}$ & & & FE & & N28 & 03 & $W 106$ & 45 & $707 C 7 A 7$ \\
\hline MN & & & & & & N27 & 08 & W105 & 41 & 10A7C7A7 \\
\hline AU & $\mathbf{A G}$ & PB & $Z N$ & & & N27 & 03 & W 105 & 15 & 4D7C7A7 \\
\hline PB & $\mathbf{A G}$ & & & & & N28 & 42 & W106 & 14 & $7 C 7 C 7 A 7$ \\
\hline$A U$ & $A G$ & & & & & N26 & 43 & W 107 & 34 & 4C7C7A 7 \\
\hline$A U$ & $A G$ & & & & & N27 & 39 & W 108 & 07 & 4C7C7A 7 \\
\hline PB & $\mathrm{ZN}$ & $A G$ & $A U$ & & & N29 & 17 & W107 & 23 & 7D7C7A7 \\
\hline AU & $A G$ & & & & & N27 & 38 & W106 & 44 & 4C7C7A7 \\
\hline PB & $\mathrm{ZN}$ & $A G$ & & $A U$ & $\mathrm{cu}$ & N3O & 17 & W 107 & 55 & 7D7C7A7 \\
\hline PB & $A G$ & & & & & N29 & 55 & W 107 & 15 & 7D7C7A7 \\
\hline$A G$ & PB & $2 \mathrm{~N}$ & $\mathrm{CU}$ & $\mathbf{F}$ & & N26 & 42 & W105 & 15 & 4D7C7A7 \\
\hline PB & $\mathbf{A G}$ & & & & & N3O & 26 & W106 & 13 & 7D 1B5A7 \\
\hline MN & & & & & & N28 & 06 & W106 & 19 & 1OA 1C7A7 \\
\hline HG & & & & & & N28 & 26 & $W 107$ & 06 & 8А7C7A7 \\
\hline $\mathrm{F}$ & BA & & & & & N26 & 37 & W 104 & 30 & $8 C 14 C 5 A 7$ \\
\hline HG & & & & & & N26 & 53 & W104 & 08 & 8A 1B7A7 \\
\hline PB & $\mathrm{ZN}$ & & & & & N29 & 05 & $W_{105}$ & 15 & 7C7C7A 7 \\
\hline$w$ & & & & & & N26 & 39 & $W 107$ & 40 & $1 A \uparrow 47 C 3 A 7$ \\
\hline AU & $\mathbf{A G}$ & & & & & N25 & 50 & $W+07$ & 02 & 4C7C7A7 \\
\hline MN & & & & & & N34 & 03 & W108 & 39 & 10A7C7A7 \\
\hline PB & $\mathrm{ZN}$ & & & $B A$ & & N28 & 39 & W105 & 20 & $7 C 1 C 2 A 7$ \\
\hline MN & & & & & & N28 & o & W105 & 49 & 1OA 1C7A7 \\
\hline PB & $A G$ & & & & & N31 & 22 & W 106 & 32 & $701 C 5 A 7$ \\
\hline$A U$ & $A G$ & & & SB & & N28 & 11 & W108 & 43 & 4C $15 C 7 A 7$ \\
\hline MN & & & & & & N28 & 19 & W104 & 57 & 10A7B7A7 \\
\hline HG & & & & & & N29 & 10 & W103 & 58 & ВА $14 C 3 A 7$ \\
\hline PB & $\mathrm{ZN}$ & AG & & $A U$ & CU $F$ & N26 & 52 & W105 & 50 & 7D 1A7A7 \\
\hline PB & $A G$ & & & & & N31 & 15 & W106 & 00 & 7D 1C7A7 \\
\hline$A U$ & $A G$ & & & & & N26 & 53 & W107 & 52 & АC $C 7 A 7$ \\
\hline MN & & & & & & N28 & 21 & W106 & 07 & 10A7C7A7 \\
\hline$A G$ & $A U$ & $\mathrm{cu}$ & & & & N3O & 05 & W107 & 45 & 4С7C $\triangle A 7$ \\
\hline$A U$ & $A G$ & & & & & N26 & 43 & W107 & 42 & 4C7C7A 7 \\
\hline AU & $A G$ & & & & & N26 & 24 & W 107 & 23 & 4С 4С उA 7 \\
\hline $\mathrm{CU}$ & & & & & & N31 & 10 & W105 & 52 & 3А $1 C 7 A 7$ \\
\hline$A G$ & $A U$ & PB & $2 N$ & & & N27 & 15 & W 105 & 34 & 4D7C7A7 \\
\hline AU & $A G$ & & & & & N26 & 22 & W 107 & 26 & 4C7C7A 7 \\
\hline MN & & & & & & N26 & 04 & W 106 & 37 & 10A7C7A7 \\
\hline $\mathrm{FE}$ & & & & & & N26 & 04 & W106 & 31 & 2A7C7A7 \\
\hline$A U$ & $\mathbf{A G}$ & & & & & N27 & 49 & $W 108$ & 50 & 4C7C7A 7 \\
\hline $\mathrm{CU}$ & & & & $\mathrm{FE}$ & & N28 & 41 & $W 104$ & 09 & 3А $7 C 7 A 7$ \\
\hline PB & $\mathrm{ZN}$ & $A G$ & & $A U$ & $\mathrm{CU}$ & N30 & 44 & W 107 & 40 & 7D57B7A7 \\
\hline$A U$ & $A G$ & & & & & N28 & 13 & W 108 & 16 & 4C7C $7 A 7$ \\
\hline AU & $A G$ & & & & & N28 & 12 & W108 & 39 & 4C7C7A7 \\
\hline PB & $\mathrm{ZN}$ & $A G$ & & $A U$ & $\mathrm{CU}$ & N26 & 48 & W 105 & 47 & 7D 1A7A7 \\
\hline PB & $2 \mathrm{~N}$ & $A G$ & SN & & & N28 & 37 & W105 & 53 & 7D 1A5A7 \\
\hline $\mathrm{CU}$ & & & & & & N26 & 13 & W107 & 12 & 3АTC7A7 \\
\hline PB & $\mathbf{A G}$ & & & & & N28 & 35 & W105 & 20 & $7014 C 3 A 7$ \\
\hline MN & & & & & & N27 & 55 & $w 106$ & 03 & 1OA7C7A7 \\
\hline PB & $\mathbf{Z N}$ & $A G$ & & $A U$ & $\mathrm{CU}$ & N27 & 52 & W105 & 30 & 7D1A5A7 \\
\hline$A G$ & PB & CU & & & & N29 & 02 & W 104 & 49 & 4D 1C7A7 \\
\hline HG & & & & & & N28 & 15 & $W 104$ & 10 & 8A $167 C 7 A 7$ \\
\hline$A U$ & $A G$ & & & & & N31 & 15 & $W 106$ & 09 & 4C 1 C7A 7 \\
\hline PB & $\mathbf{A G}$ & & & & & N3O & 40 & $W 106$ & 05 & 7D147C7A7 \\
\hline PB & $\mathbf{A G}$ & $\mathrm{CU}$ & ZN FE & & & N3O & 40 & W106 & 20 & 4D 147C7A7 \\
\hline PB & $\mathrm{ZN}$ & $A U$ & & & & N29 & 35 & W105 & 48 & 7D $14 C 3 A 7$ \\
\hline PB & $\mathrm{ZN}$ & $A G$ & & & & N3O & 22 & W106 & 26 & 7D $1 C 7 A 7$ \\
\hline PB & $A G$ & & & & & N3O & 46 & W106 & 14 & 7D 1C7A7 \\
\hline
\end{tabular}




\begin{tabular}{|c|c|c|}
\hline $\mathrm{M} \times \mathrm{CH}$ & 62 & SIERRA LA MOJINA \\
\hline $\mathrm{M} \times \mathrm{CH}$ & 94 & SIERRA PLACER DE GUADALUPE \\
\hline $\mathrm{M} \times \mathrm{CH}$ & 23 & STO. DOMINGO \\
\hline $\mathrm{MXCH}$ & 181 & TALAMANTES \\
\hline $\mathrm{M} \times \mathrm{CH}$ & 129 & TEMORIS \\
\hline $\mathrm{M} \times \mathrm{CH}$ & 67 & TERRENATES \\
\hline $\mathrm{M} \times \mathrm{CH}$ & 90 & TOSISIHUA \\
\hline $\mathrm{M} \times \mathrm{CH}$ & 146 & TUBARES \\
\hline $\mathrm{M} \times \mathrm{CH}$ & 123 & URACHIC \\
\hline $\mathrm{M} \times \mathrm{CH}$ & 148 & URI QUE \\
\hline $\mathrm{MXCH}$ & 43 & V. AHUMADA-LOS LAMENTOS A \\
\hline $\mathrm{M} \times \mathrm{CH}$ & 172 & VIRGINIA \\
\hline $\mathrm{MXCH}$ & 4 & WILKIE \\
\hline $\mathrm{M} \times \mathrm{CH}$ & 59 & YOQUIVO \\
\hline $\mathrm{M} \times \mathrm{CH}$ & 190 & ZAPOTE \\
\hline $\mathrm{MXCH}$ & 36 & ZONA DE CONTENCION \\
\hline $\mathrm{MXCH}$ & 144 & ZONA VALLE DE OLIVOS \\
\hline $\mathrm{MXCL}$ & 6 & CERRO NAHUATL \\
\hline$M \times C L$ & 4 & COYUTLAN \\
\hline $\mathrm{MXCL}$ & 2 & LOS CORDONES \\
\hline $\mathrm{M} \times \mathrm{CL}$ & 3 & MANZANILLO \\
\hline$M \times C L$ & 5 & MESA DE FIERRO \\
\hline$M \times C L$ & 1 & PENA COLORADO \\
\hline$M \times C L$ & 7 & PISCILA \\
\hline $\mathrm{M} \times \mathrm{CO}$ & 1 & AGUACHILE \\
\hline$M \times C O$ & 54 & ALFA \\
\hline$M \times C O$ & 53 & BANUELOS \\
\hline $\mathrm{MXCO}$ & 42 & BIZNAGA Y BACON \\
\hline $\mathrm{M} \times \mathrm{CO}$ & 34 & CANALONES VOLADERO, AGUI JAN 77 \\
\hline $\mathrm{MxCO}$ & 30 & CANDELA \\
\hline$M \times C O$ & 5 & CERRO DE LA VASCA \\
\hline$M \times C O$ & 36 & CERRO DE LOS INDIOS \\
\hline MXCO & 18 & DELICIAS \\
\hline $\mathrm{M} \times \mathrm{CO}$ & 4 & EL ALAZAN \\
\hline $\mathrm{M} \times \mathrm{CO}$ & 52 & EL CAPULIN \\
\hline $\mathrm{M} \times \mathrm{CO}$ & 10 & EL HUARACHE \\
\hline$M \times C O$ & 21 & EL LUCERO \\
\hline MXCO & 47 & EL NINO, CABALLOS \\
\hline $\mathrm{M} \times \mathrm{CO}$ & 28 & EL PANUCO \\
\hline $\mathrm{M} \times \mathrm{CO}$ & 33 & ESPERANZA Y FORTUNA, LEON DOS \\
\hline $\mathrm{MXCO}$ & 41 & EUREKA \\
\hline$M \times C O$ & 11 & GOMEZ \\
\hline $\mathrm{M} \times \mathrm{CO}$ & 48 & GRAL. CEPEDA \\
\hline MXCO & 9 & HERCULES \\
\hline$M \times C O$ & 24 & HERMANAS \\
\hline$M \times C O$ & 14 & LA ALCANFORADA \\
\hline$M \times C O$ & 6 & LA ENCANTADA-BUENAVISTA \\
\hline $\mathrm{MXCO}$ & 7 & LA MARIPOSA \\
\hline MXCO & 32 & LA REFORMA \\
\hline $\mathrm{M} \times \mathrm{CO}$ & 57 & LA VICTORIA \\
\hline $\mathrm{M} \times \mathrm{CO}$ & 17 & LAGUNA DEL REY \\
\hline$M \times C O$ & 23 & LUCERO Y BALUARTE \\
\hline$M \times C O$ & 22 & MINA HONDA \\
\hline $\mathrm{M} \times \mathrm{CO}$ & 15 & MINAS DE LA MULA \\
\hline MXCO & 12 & MINAS DEL TULE \\
\hline$M \times C O$ & 44 & MINERAL DE PALOMAS \\
\hline$M \times C O$ & 27 & NAVARETE \\
\hline$M \times C O$ & 50 & P.V. 14-VENADOS \\
\hline$M \times C O$ & 40 & P.V.17-DOLORES \\
\hline $\mathrm{MxCO}$ & 43 & P.V. 27 \\
\hline
\end{tabular}

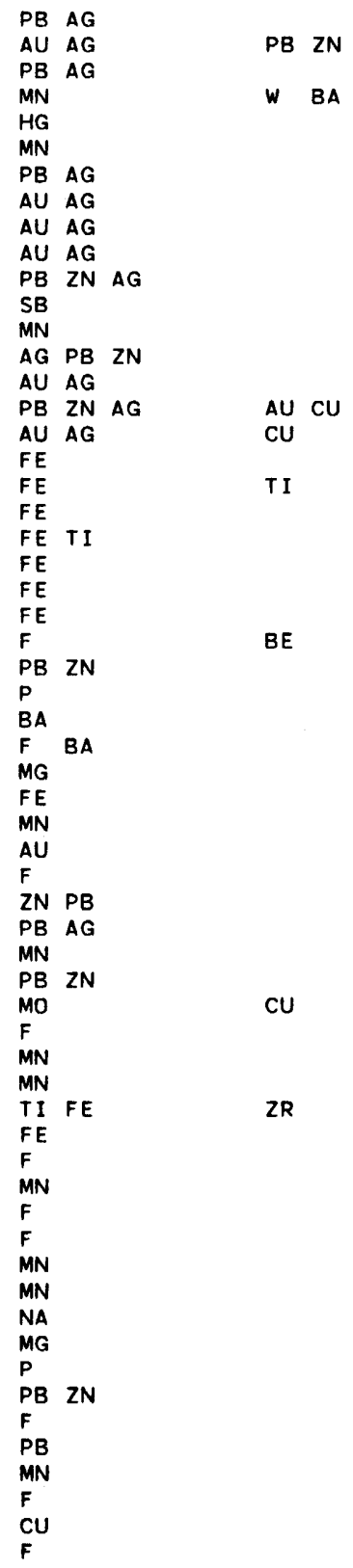

\begin{tabular}{|c|c|c|c|c|}
\hline 29 & 56 & W106 & 50 & $70107 A 7$ \\
\hline N29 & 20 & W105 & 24 & 4C $107 A 7$ \\
\hline N3O & 37 & $W 106$ & 40 & 707C7A7 \\
\hline N26 & 56 & W105 & 26 & 1OA7B7A7 \\
\hline N27 & 17 & W108 & 16 & BA7C $7 A 7$ \\
\hline N29 & 37 & W106 & 47 & 1OA7B7A7 \\
\hline N29 & 47 & W105 & 22 & $7 D 14 C 3 A 7$ \\
\hline N26 & 57 & $W 107$ & 01 & $4 C 7 C 7 A 7$ \\
\hline N27 & 52 & $W 108$ & 12 & 4C7C7A7 \\
\hline N27 & 14 & $W 107$ & 55 & 4C7C7A 7 \\
\hline N3O & 31 & W105 & 47 & $70185 A 7$ \\
\hline N27 & 10 & W105 & 57 & $8 B 15 C 7 A 7$ \\
\hline N3O & 09 & $W 108$ & 13 & 10A7C7A7 \\
\hline N28 & 09 & W108 & 12 & $407 C 7 A 7$ \\
\hline N25 & 54 & W107 & 14 & 4C7C7A 7 \\
\hline N3O & 08 & W106 & 45 & 7D 14C3A7 \\
\hline N27 & 13 & W106 & 15 & 4C7C7A7 \\
\hline N19 & 10 & W103 & 54 & $2 A 146 C 3 A 6$ \\
\hline N18 & 55 & W104 & 03 & $2 A 37 C 4 A 8$ \\
\hline N19 & 07 & W104 & 12 & $2 A 146 C 3 A 6$ \\
\hline N19 & 03 & W104 & 19 & $2 C 37 C 4 A 8$ \\
\hline N19 & 18 & $W 103$ & 52 & $2 A 146 C 3 A 6$ \\
\hline N19 & 22 & W104 & 05 & 2A $146 A 3 A G$ \\
\hline N19 & 09 & W103 & 43 & $2 A 146 C 3 A 6$ \\
\hline N29 & 15 & $W 102$ & 32 & $8 C 14 B 5 A 7$ \\
\hline N25 & 02 & $W 100$ & 59 & $701 C 7 A 7$ \\
\hline N25 & 05 & W101 & 10 & $2 E_{1} 1 C_{2 A 4}$ \\
\hline N25 & 45 & W101 & 37 & 8D 1C $10 A 5$ \\
\hline N26 & 05 & $W 101$ & 40 & $8 C 1 A 5 A 7$ \\
\hline N26 & 45 & $W 100$ & 45 & $6 D 1 C 2 A 6$ \\
\hline N28 & 40 & $W 102$ & 47 & $2 A 14 C 3 A 7$ \\
\hline N25 & 53 & $W 102$ & 20. & 1OA 1C7A7 \\
\hline N26 & 18 & $W 102$ & 52 & $\triangle A 3 C 4 A 8$ \\
\hline N28 & 45 & W103 & 10 & 8C 1C7A7 \\
\hline N25 & 08 & W 101 & 15 & 7D 1C7A7 \\
\hline N28 & 02 & $W 103$ & 43 & $7014 C 7 A 7$ \\
\hline N27 & 43 & $W 101$ & 28 & 1OA7C7A7 \\
\hline N25 & 15 & W101 & 00 & $701 C 7 A 7$ \\
\hline N26 & 48 & W101 & 10 & $3 B 4 C 1 A 7$ \\
\hline N26 & 25 & $W 101$ & 40 & $8 C 1 A 5 A 7$ \\
\hline N25 & 50 & $W 101$ & 29 & 10A7C7A7 \\
\hline N29 & 13 & W101 & 04 & 1OA7C7A7 \\
\hline N25 & 23 & W 101 & 30 & 2C 1B4A5 \\
\hline N28 & 02 & W103 & 49 & $2 A 4 C 7 A 7$ \\
\hline N27 & 15 & W 101 & 10 & $8 C 1 C 5 A 7$ \\
\hline N27 & 47 & $W 103$ & 42 & 1OA 1C7A7 \\
\hline N28 & 37 & $W 102$ & 22 & 8C 14A5A7 \\
\hline N28 & 10 & $W 102$ & $\infty$ & $8 C 14 C 5 A 7$ \\
\hline N26 & 40 & W101 & 40 & 1OA IC7AT \\
\hline N24 & 52 & $W 101$ & 35 & 10A7C7A7 \\
\hline N27 & 00 & W103 & 25 & 6B37B6A8 \\
\hline N27 & 15 & $W 101$ & 15 & 6D 1C2A6 \\
\hline N27 & 44 & W101 & $\infty$ & 2E $1 C 2 A 4$ \\
\hline N27 & 30 & $W 102$ & 33 & $7 D 14 C 3 A 7$ \\
\hline N28 & 29 & $W 101$ & 35 & $8 C 14 A 5 A 7$ \\
\hline N25 & 37 & $W 100$ & 47 & 7D 1C7A7 \\
\hline N26 & 52 & W 101 & 30 & 10A7C7A7 \\
\hline N25 & 09 & W101 & 48 & $8 C 1 C 7 A 7$ \\
\hline N24 & 54 & $W 102$ & 50 & 3A IC $10 A 7$ \\
\hline N25 & 45 & W 101 & 27 & BC $1 C 7 A 7$ \\
\hline
\end{tabular}




\begin{tabular}{|c|c|c|}
\hline $\mathrm{M} \times \mathrm{CO}$ & 39 & P.V.3-SIERRA DEL MIMBRE \\
\hline $\mathrm{M} \times \mathrm{CO}$ & 38 & P.V.3-VILLA BILBAO \\
\hline $\mathrm{M} \times \mathrm{CO}$ & 46 & PAME \\
\hline$M \times C O$ & 29 & PANUCO \\
\hline $\mathrm{M} \times \mathrm{CO}$ & 49 & PV. 12 \\
\hline $\mathrm{MXCO}$ & 45 & RAMOS ANISPE \\
\hline MXCO & 8 & RANCHO PROGRESO \\
\hline $\mathrm{M} \times \mathrm{CO}$ & 31 & REAL VIEJOS \\
\hline $\mathrm{M} \times \mathrm{CO}$ & 25 & SAN JUAN \\
\hline $\mathrm{M} \times \mathrm{CO}$ & 3 & SANTA ANITA, EL MELON \\
\hline $\mathrm{MxCO}$ & 13 & SANTA ELENA \\
\hline $\mathrm{M} \times \mathrm{CO}$ & 56 & SANTA MARIA \\
\hline $\mathrm{MxCO}$ & 26 & SANTA RITA \\
\hline $\mathrm{MxCO}$ & 20 & SANTA ROSA \\
\hline $\mathrm{M} \times \mathrm{CO}$ & 35 & SIERRA DE LA PAILA \\
\hline $\mathrm{MxCO}$ & 37 & SIERRA DE PARRAS \\
\hline $\mathrm{M} \times \mathrm{CO}$ & 2 & SIERRA DE SAN VICENTE \\
\hline$M \times C O$ & 55 & SIERRA GOME $Z$ FARIAS-CARBONERAS \\
\hline Mxco & 51 & SIERRA LA CATANA \\
\hline $\mathrm{M} \times \mathrm{CO}$ & 16 & SIERRA MOJADA \\
\hline $\mathrm{M} \times \mathrm{CO}$ & 19 & VENADOO \\
\hline$M \times C P$ & 10 & ARRI AGA \\
\hline$M \times C P$ & 9 & CERRO COLORADO O AURORA \\
\hline$M \times C P$ & 7 & CINTALAPA \\
\hline MXCP & 1 & ESTACION JUAREZ \\
\hline MXCP & 5 & IXTAPA \\
\hline MXCP & 4 & JICOTAL \\
\hline MXCP & 15 & LAJERIA \\
\hline$M \times C P$ & 12 & MONTE BELLO \\
\hline MXCP & 14 & NUEVA MORELIA \\
\hline$M \times C P$ & 16 & PACAYAL \\
\hline$M \times C P$ & 13 & PIPIUIAPAN \\
\hline MXCP & 3 & SABANILLA \\
\hline$M \times C P$ & 2 & SANTA FE \\
\hline MXCP & 11 & SANTA MARGARITA \\
\hline$M \times C P$ & 8 & TAPANATEPEC-PLATANILLO \\
\hline MXCP & 17 & TOL IMAN \\
\hline $\mathrm{M} \times \mathrm{CP}$ & 6 & TUXTLA GUTIERREZ \\
\hline MXDR & 46 & $\triangle D E L A$ \\
\hline MXDR & 64 & ALAMITO \\
\hline MXDR & 54 & AMERICA-SAPIORIS \\
\hline MXDR & 44 & AREA DE PERICOS \\
\hline MXDR & 98 & ARROYO DE LA HIGUERA \\
\hline MXDR & 10 & AZUFRE TLAHUALILO \\
\hline MXDR & 16 & CANELAS \\
\hline MXDR & 102 & CEBOLLAS \\
\hline MXOR & 103 & CERRO DE LAS GALLINAS \\
\hline MXDR & 105 & CERRO DE SACRIFICIOS \\
\hline MXDR & 74 & CERRO DEL MERCADO \\
\hline MXDR & 1 & CERRO GORDO, SAN FERMIN \\
\hline MXOR & 18 & CERRO PRIETO \\
\hline MXDR & 36 & CERRO PRIETO Y CERRO BLANCO \\
\hline MXDR & 77 & COYOTERA \\
\hline MXDR & 94 & CRUZ DE MAYO \\
\hline MXDR & 53 & CUATRO REALES, EL APACHE \\
\hline MXDR & 82 & CUENCAME \\
\hline MXDR & 83 & CUENCAME \\
\hline MXDR & 33 & DESCUBRIDORA \\
\hline MXDR & 81 & DTO. CUENCAME, VELARDENA, PEDF \\
\hline MXDR & 58 & DTO. EL SALITRE \\
\hline
\end{tabular}

\begin{tabular}{|c|c|c|c|c|c|c|c|c|}
\hline $\mathrm{cU}$ & & & & N25 & $\infty$ & $W 103$ & 08 & 3A1C7A7 \\
\hline $\mathbf{A G}$ & PB & $\mathrm{cu}$ & & N25 & 26 & $W 102$ & 50 & $401 C_{10 A 7}$ \\
\hline PB & $2 \mathrm{~N}$ & & & N25 & 25 & W100 & 50 & $701 C 7 A 7$ \\
\hline$A U$ & $A G$ & $\mathrm{cu}$ & & N26 & 44 & $w 101$ & 00 & 4C7C7A7 \\
\hline BA & & & & N25 & 11 & W101 & 37 & $8 D 1 C 7 A 7$ \\
\hline SR & & & & N25 & 33 & $W 100$ & 52 & BE $1 C 7 A 6$ \\
\hline $\mathrm{FE}$ & & & & N28 & 17 & $W 103$ & 39 & $2 A 4 C 7 A 7$ \\
\hline $\mathrm{FE}$ & & & & N26 & 45 & W101 & 35 & $2 A+4 C 3 A 7$ \\
\hline P & & & & N27 & 15 & $W 101$ & 05 & $2 E+C 2 A 4$ \\
\hline $\mathrm{F}$ & & & & N28 & 58 & $W 102$ & 55 & $8 C+B 7 A 7$ \\
\hline PB & $\mathrm{Cu}$ & & & N27 & 55 & W103 & 40 & $7 D 15 C 7 A 7$ \\
\hline BA & & & & N24 & 52 & W101 & 10 & $8 D 1 C 2 A 5$ \\
\hline BA & & & & N27 & 07 & W101 & 30 & 8D 1B7A7 \\
\hline BA & & & & N27 & 50 & W101 & 36 & BD 1B7A7 \\
\hline MN & & & & N25 & 58 & $W_{102}$ & 03 & 1OA $1 C 7 A 7$ \\
\hline$F$ & & & & N25 & 27 & W102 & 18 & 8C $1 A 5 A 7$ \\
\hline $\mathbf{F}$ & & & & N29 & 00 & $W 102$ & 03 & $8 C 1 C 7 A 7$ \\
\hline P & & & & N24 & 55 & $W 100$ & 57 & 2E 1B2A4 \\
\hline P & & & & N25 & 11 & W101 & 19 & 2E 1B2A4 \\
\hline PB & $\mathrm{CU}$ & & & N27 & 18 & W103 & 45 & 7D14B5A7 \\
\hline MN & & & & N26 & 04 & $W 102$ & 40 & 10A $1 C 7 A 7$ \\
\hline FE & & & $\mathrm{CU}$ & $N+6$ & 15 & W093 & 52 & $2 D 145 C 3 A 7$ \\
\hline FE & & & $\mathrm{CU}$ & N16 & 17 & W093 & 58 & 2D4C7A7 \\
\hline BA & & & & $\mathrm{N} 16$ & 42 & W093 & 45 & 8D 1C1OA5 \\
\hline$A L$ & & & & N17 & 42 & W093 & 11 & 10BЗС $9 A 6$ \\
\hline CU & & & PB ZN & $\mathrm{N} 16$ & 50 & W092 & 55 & $3 A 14 C 3 A 7$ \\
\hline LI & & & & $\mathrm{N} 17$ & 01 & W092 & 50 & 1E $4 C 1 A 4$ \\
\hline PB & $\mathrm{ZN}$ & & & N15 & 40 & WO92 & 05 & $7015 C 7 A 7$ \\
\hline$A L$ & & & & N16 & 10 & W091 & 45 & 1OB23C9A7 \\
\hline$A G$ & PB & $Z N$ & $\triangle U C U$ & N15 & 44 & WO92 & 18 & $4015 C 7 A 7$ \\
\hline$A G$ & PB & $\mathrm{ZN}$ & $A U C U$ & N15 & 38 & W092 & $\infty$ & $4 D 15 C 7 A 7$ \\
\hline AG & PB & $2 \mathrm{~N}$ & $A U C U$ & N15 & 40 & W093 & 13 & $4015 C 7 A 4$ \\
\hline $\mathrm{CU}$ & & & & N17 & 20 & WO92 & 42 & 3A $1 C 7 A 7$ \\
\hline $\mathrm{CU}$ & $A U$ & $\Delta G$ & & N17 & 24 & W093 & 03 & ЗE $147 C 3 A 7$ \\
\hline MG & & & & N17 & 26 & W091 & 34 & $6 D 2 C 2 A 5$ \\
\hline $\mathrm{CU}$ & & & FE & N16 & 26 & W094 & $\infty$ & 3A147C5A7 \\
\hline CU & & & & N15 & 20 & W092 & 12 & $3 A 145 C 5 A 4$ \\
\hline MG & & & & N16 & 45 & W093 & 10 & 6D 1 C2A5 \\
\hline$A G$ & PB & & & N25 & 25 & W104 & 17 & 4D $47 C 7 A 7$ \\
\hline$A G$ & PB & $A U$ & & N24 & 46 & W105 & 06 & 4D7C7A7 \\
\hline SN & & & & N25 & 08 & $W 104$ & 54 & 1B7C7A7 \\
\hline $\mathrm{FE}$ & & & $\mathrm{CU}$ & N25 & 28 & $W 104$ & 32 & $2 A 14 C 3 A 7$ \\
\hline$A U$ & $\Delta G$ & & & N23 & 42 & W105 & 50 & 4C $4 B 7 A 7$ \\
\hline$s$ & & & & N26 & 15 & W103 & 30 & 11A 1C5A7 \\
\hline$\Delta U$ & $A G$ & & & N25 & 07 & $W 106$ & 31 & 4C7B $7 A 7$ \\
\hline$A G$ & PB & $2 \mathrm{~N}$ & $A U C U$ & N23 & 20 & W104 & 45 & $4 D 1 B 7 A 7$ \\
\hline SN & & & & N23 & 16 & W104 & 10 & 1B7B7A7 \\
\hline$A G$ & $\mathrm{CU}$ & & & N23 & 53 & W103 & 55 & $4014 C 3 A 7$ \\
\hline $\mathrm{FE}$ & & & & N24 & $\mathrm{O} 2$ & W104 & 40 & 2A7A5A 7 \\
\hline $\mathbf{F}$ & & & & N26 & 23 & W104 & 48 & $8 C 1 C 7 A 7$ \\
\hline AU & $A G$ & & & N25 & 01 & $W 106$ & 30 & 4C7C7A7 \\
\hline BA & & & SB & N25 & 45 & W104 & 13 & 8D 14C5A7 \\
\hline$A G$ & PB & & & N25 & 39 & W103 & 57 & 4D 1 C5A7 \\
\hline SB & & & & N24 & 08 & W103 & 52 & $8 B 7 C 7 A 7$ \\
\hline $\mathbf{F}$ & & & & N25 & 11 & W104 & 44 & $8 C 7 C 7 A 7$ \\
\hline HG & & & & N24 & 53 & W103 & 32 & $8 A 1 B 7 A 7$ \\
\hline MN & & & & N24 & 48 & W103 & 42 & 1OA $1 C 7 A 7$ \\
\hline $\mathrm{CU}$ & & & & N25 & 59 & $W 104$ & 14 & 3А 14С3А7 \\
\hline $\mathbf{A G}$ & PB & $Z N$ & $A U C U$ & N25 & 05 & W103 & 40 & 4D $17 B 5 A 7$ \\
\hline$A G$ & PB & & & N25 & 08 & W105 & 20 & 4D7C7A7 \\
\hline
\end{tabular}




\begin{tabular}{|c|c|c|c|c|c|c|c|c|c|c|c|}
\hline MXDR & 42 & DTO. PICACHO DE CANDELA & MN & & & & N25 & 30 & W105 & 32 & 10А7B7A7 \\
\hline MXDR & 75 & DTO. SAN DIMAS (TAYOLTITA) & AU & $\mathbf{A G}$ & & & N24 & 10 & W105 & 55 & 4C7A7A6 \\
\hline MXDR & 5 & EL AGUILA (Z. EL CARMEN) & AG & CU & & & N26 & 12 & W105 & 50 & 407C7A7 \\
\hline MXDR & 92 & EL CABALLO & SB & & & $H G$ & N24 & 28 & W103 & 49 & 8B7C7A7 \\
\hline MXDR & 4 & EL COBRE & $\mathrm{CU}$ & & & & N26 & 15 & W105 & 22 & 3А47C7A7 \\
\hline MXDR & 9 & EL CONEJO & $\mathrm{s}$ & & & & N26 & 16 & W103 & 50 & 11A1C10A7 \\
\hline MXDR & 57 & EL CRESTON & $A G$ & PB & ZN & & N25 & 16 & W105 & 47 & 407C7A7 \\
\hline MXDR & 6 & EL CUARENTA & HG & & & & N26 & 08 & W105 & 32 & 8А7B7A7 \\
\hline MXDR & 96 & EL LIMONCITO ( $Z$. MALA NOCHE) & $\mathbf{A G}$ & & & AU & N23 & 59 & W105 & 35 & АC7B7A7 \\
\hline MXDR & 66 & EL LUCERO, EL PORVENIR, ETC. & SN & & & & N24 & 33 & W104 & 47 & $187 C 7 A 7$ \\
\hline MXDR & 50 & EL MEZQUITE & u & TH & & & N25 & 15 & W104 & 40 & 9E7C7A7 \\
\hline MXDR & 22 & EL RODEO & AU & & & $\mathrm{CU}$ & N24 & 48 & $w 106$ & 43 & 4B7C7A7 \\
\hline MXDR & 73 & EL SAGAL & SB & & & & N24 & 12 & W104 & 20 & $8 B 7 C 7 A 7$ \\
\hline MXDR & 38 & EL TREBOL & $A G$ & PB & & & N25 & 40 & W104 & 30 & 4D $1 C 7 A 7$ \\
\hline MXDR & 19 & EL TUNEL ( $Z$. SAN MIGUEL DEL CANTIL.) & $A U$ & AG & & PB & N24 & 57 & W106 & 15 & 4C7B7A7 \\
\hline MXDR & 88 & EL ZORRILLO & SB & & & & N24 & 30 & W103 & 07 & 8B7C7A7 \\
\hline MXDR & 34 & EVANGELINA & SB & & & & N25 & 53 & $W 104$ & 31 & $8 B 7 C 7 A 7$ \\
\hline MXDR & 86 & FALLA AL ORIENTE DE LUNA LLENA & $\mathbf{A G}$ & $\mathrm{CU}$ & & & N24 & 51 & W102 & 31 & 4D 1C7A7 \\
\hline MXDR & 101 & GARIBAYA & $A U$ & AG & CU PB ZN & & N23 & 22 & W105 & 22 & 4D7C7A7 \\
\hline MXDR & 29 & GUANACEVI & AU & $\mathbf{A G}$ & & & N25 & 35 & $w 105$ & 58 & 4C7B 7A7 \\
\hline MXDR & 104 & JACUIXTLE (LLUVIA DE ORO) & AU & & & & N23 & 10 & W105 & 20 & 4A7C7A7 \\
\hline MXDR & 27 & LA ALIANZA & $\mathbf{A G}$ & cu & PB & & N24 & 23 & w106 & 31 & 4D7C7A7 \\
\hline MXDR & 63 & LA BARROSA & SN & & & & N24 & 50 & W104 & 48 & 1B7C7A7 \\
\hline MXDR & 100 & LA CANDELARIA & $\mathbf{A G}$ & $A U$ & PB & & N23 & 25 & W105 & 23 & 4D4C7A7 \\
\hline MXDR & 15 & LA COBRIZA Y AMPLIACIONES & $\mathbf{A G}$ & PB & $\mathrm{ZN}$ & & N25 & 15 & W106 & 22 & 4D7C7A7 \\
\hline MXDR & 37 & LA COLORADA & $\mathbf{A G}$ & PB & & CU & N25 & 43 & W104 & 20 & 4D 1C7A7 \\
\hline MXDR & 79 & LA ESPERANZA & $\mathbf{A G}$ & $\mathrm{Cu}$ & & & N25 & 28 & W103 & 35 & 407C7A7 \\
\hline MXDR & 20 & LA FE & $A U$ & AG & & PB & N24 & 54 & W106 & 30 & 4D5C7A7 \\
\hline MXDR & 67 & LA FORTUNA-EL SOLDADO & SN & & & & N24 & 30 & W105 & $\infty$ & 1B7C7A7 \\
\hline MXDR & 2 & LA MADRUGADA & $A U$ & $A G$ & PB & & N26 & 15 & W105 & 45 & 4C7C7A7 \\
\hline MXDR & 21 & LA MELESIA ( $z$. SAN ANDRES DE LA SIERRA) & $\mathbf{A G}$ & PB & $\mathrm{ZN}$ & AU CU & N24 & 54 & $W 106$ & 17 & 4D5B7A7 \\
\hline MXDR & 76 & LA OJUELA & PB & $\mathbf{Z N}$ & AG & $A U C U$ & N25 & 50 & W103 & 50 & 7D $1 A 5 A 7$ \\
\hline MXDR & 70 & LA PALMA & $F$ & & & & N24 & 35 & W104 & 03 & BC 1 C5A7 \\
\hline MXDR & 47 & LA PRECIOSA & u & & & & N25 & 21 & W104 & 04 & 9A 1C5A7 \\
\hline MXDR & 59 & LA PROVIDENCIA, ONTARIO ETC. & AU & $A G$ & PB & & N25 & 03 & W105 & 33 & 4C7C7A7 \\
\hline MXDR & 93 & LA PURISIMA & MN & & & & N24 & 15 & $w 103$ & 45 & 10A7C7A7 \\
\hline MXDR & 45 & LA SANTA CRUZ & AU & $\Delta G$ & MO $Z \mathrm{~N}$ & & N25 & 30 & $W 104$ & 07 & 4D 1C7A7 \\
\hline MXDR & 43 & LA VIRGINIA & SB & & & & N25 & 26 & W104 & 57 & $8 B 7 C 7 A 7$ \\
\hline MXDR & 78 & LA ZARNOSA & MN & & & & N25 & 37 & $w 103$ & 40 & 1OA $\{4 B 10 A 7$ \\
\hline MXDR & 12 & LAJAS & MN & & & & N25 & 35 & W106 & 15 & 10A7C7A7 \\
\hline MXDR & 8 & LAS COLORADAS & $\mathbf{A G}$ & AU & PB ZN & & N26 & 03 & W105 & 17 & $404 C 7 A 7$ \\
\hline MXDR & 23 & LAS PALOMAS & $\widehat{A G}$ & & & & N24 & 45 & W106 & 25 & 4D7C7A7 \\
\hline MXDR & 80 & LAS TRANCAS & AG & PB & & & N25 & 12 & W103 & 40 & 4D 1C7A7 \\
\hline MXDR & 28 & LOS GUEROS DE S. FERNANDO & AU & AG & PB ZN CU & & N24 & 20 & W106 & 03 & 4D4C7A7 \\
\hline MXDR & 60 & LOS PATOS Y ARRIETA & $\mathrm{MN}$ & & & & N25 & 07 & W105 & 00 & 10A7C7A7 \\
\hline MXDR & 72 & M. NIAGARA & $\mathbf{F}$ & & & & N24 & 05 & $W 104$ & 02 & $8 C 7 C 7 A 7$ \\
\hline MXDR & 91 & MINA SONRISA & HG & & & & N24 & 38 & W103 & 42 & 8А7C7A7 \\
\hline MXDR & 3 & MURC I ELAGOS & $\mathbf{A G}$ & PB & ZN & $\mathrm{AU} C U$ & N26 & 15 & W105 & 31 & 4D47C7A7 \\
\hline MXDR & 31 & NAVIDAD & $\mathbf{F}$ & & & & N25 & 54 & W105 & 08 & BC7C7A7 \\
\hline MXDR & 95 & OCHOA & SN & & & & N24 & 02 & W103 & 55 & 1B7C7A7 \\
\hline$M X D R$ & 32 & OLIVIA & SB & & & & N25 & 58 & $W 104$ & 23 & 8B7C7A7 \\
\hline MXDR & 68 & OTAEZ & $\mathbf{A G}$ & PB & $\mathrm{ZN}$ & $A U C U$ & N24 & 34 & W105 & 54 & 4D7B7A7 \\
\hline MXDR & 69 & PARAISO & $A L$ & & & & N24 & 40 & $w 104$ & $\infty$ & 10B7C5A7 \\
\hline MXDR & 41 & PENA DEL GRINGO & $\mathbf{A G}$ & & & & N25 & 37 & W104 & 12 & 4D 15C7A7 \\
\hline$M \times D R$ & 49 & PESCADORES & $\widehat{A G}$ & PB & $\mathrm{cu}$ & & N25 & 23 & W104 & 47 & 4D4C7A7 \\
\hline MXDR & 56 & PROMONTORIO & $A U$ & $\mathbf{A G}$ & & & N25 & 14 & W105 & 08 & $4 C 7 C 7 A 7$ \\
\hline MXDR & 62 & REYNA ISABEL & AU & $\widehat{A G}$ & PB & & N24 & 53 & W104 & 45 & 4C $15 C 7 A 7$ \\
\hline MXDR & 51 & RODEO & $w$ & HG & & & N25 & 10 & W104 & 32 & 1A7C7A7 \\
\hline$M \times D R$ & 84 & SAN EOUARDO & $A U$ & $\mathbf{A G}$ & & & N24 & 50 & W103 & 56 & 4C 14B7A7 \\
\hline MXDR & 40 & SAN FRANCISCO DE LOS POBRES & SB & & & & N25 & 38 & W104 & 23 & $8 B 7 C 7 A 7$ \\
\hline
\end{tabular}




\begin{tabular}{|c|c|c|}
\hline MXDR & 52 & SAN GABRIEL \\
\hline MXDR & 25 & SAN JACOBO ( $Z$. SIERRA SANTA) \\
\hline MXDR & 99 & SAN JOSE DE LA PARRILLA \\
\hline MXDR & 89 & SAN JOSE DE REYES \\
\hline MXDR & 11 & SAN JOSE DEL DESIERTO \\
\hline MXDR & 87 & SAN JUAN DE GUADALUPE \\
\hline$M \times D R$ & 65 & SAN LUCAS \\
\hline MXDR & 7 & SAN PEDRO \\
\hline MXDR & 48 & SANTA BARBARA \\
\hline MXDR & 39 & SANTA ISABEL \\
\hline MXDR & 13 & SANTA RITA \\
\hline MXDR & 30 & SANTA MARIA DEL ORO \\
\hline MXDR & 35 & SANTO NINO, LOS VICTORINOS \\
\hline$M \times D R$ & 85 & SIERRA DE RAMIREZ \\
\hline MXDR & 17 & TAMAZULA \\
\hline MXDR & 97 & TEBICOS \\
\hline MXDR & 61 & VILLEGAS \\
\hline MXDR & 26 & Z. RINCON DE NEVAREZ \\
\hline MXDR & 24 & Z. SAN PEDRO DE AZAFRANES \\
\hline MXDR & 71 & ZONA AVINO \\
\hline$M \times D R$ & 90 & ZONA CUENCAME \\
\hline MXDR & 14 & ZONA MINERA LA SOLEDAD \\
\hline MXDR & 55 & ZONA SIERRA DE SAN FRANCISCO \\
\hline MXEM & 4 & ALBARRAN \\
\hline MXEM & 3 & LA GUADALUPANA Y OTROS \\
\hline MXEM & 1 & TEMASCALTEPEC \\
\hline MXEM & 2 & TIERRA COLORADA \\
\hline MXGN & 19 & ALLENDE \\
\hline MXGN & 6 & ARCELIA \\
\hline MXGN & 24 & ATARUEA \\
\hline MXGN & 9 & CAPETILLO \\
\hline MXGN & 14 & CERRO DE LA FAJA \\
\hline MXGN & 15 & CERRO SAN ANTONIO \\
\hline MXGN & 4 & EL RIALITO DISTRICT \\
\hline MXGN & 20 & EL ROBLE, REGINA ET AL \\
\hline MXGN & 17 & GUANAJUATO (LA VALENCIANA ETC.) \\
\hline MXGN & 16 & LA PROTECTORA ET AL \\
\hline MXGN & 12 & LA VICTORIA ET AL \\
\hline MXGN & 1 & MELCHOR \\
\hline MXGN & 23 & MINA DE JESUS \\
\hline MXGN & 18 & MINA LA TAPONA \\
\hline MXGN & 11 & POZOS \\
\hline MXGN & 3 & SAN DIEGO DE LA UNION \\
\hline MXGN & 8 & SAN JOSE DE TANQUE \\
\hline MXGN & 22 & SAN MARTIN \\
\hline MXGN & 2 & TLACHIQUERO \\
\hline MXGN & 10 & TRANCAS \\
\hline MXGN & 5 & XICHU (ZONA MAJADA DE ESPIRITU SANTO) \\
\hline MXGN & 21 & ZAMORANO \\
\hline MXGN & 13 & ZONA BONANZA \\
\hline MXGN & 7 & ZONA PROVIDENCIA \\
\hline MXGR & 55 & ARROYO LA LIMA \\
\hline MXGR & 35 & BALSAS-LIMON GUADALUPE \\
\hline MXGR & 45 & BARRANCA AHUEHUETLA \\
\hline MXGR & 34 & BARRANCA DE CUBA \\
\hline MXGR & 41 & BARRANCA DE LOS NOGALES \\
\hline MXGR & 40 & CAMOTLA \\
\hline MXGR & 13 & CAMPO MORADO GP. (REFORMA ET AL) \\
\hline MXGR & 38 & CARMEN Y CONCEPCION \\
\hline MXGR & 43 & CERRO DE EPAZOTE \\
\hline
\end{tabular}

SB

$A G \quad P B \quad Z N$

$\mathrm{AU} \mathrm{CU}$

$A G \quad P B \quad Z N$

$\mathrm{AU} \mathrm{CU}$

$H G$

AG

$A G$ PB ZN

$A U A$

SB

$A G$ PB ZN

$A U$
$A G C U$

MN

$\begin{array}{ll}A U & A G \\ A U & A G\end{array}$

SN

$S B$

CU MO

$A G$ AU

AU $P B$

PB ZN AG

$A G$

SB $A G$

AU $A G$

HG AG

$S N$

AU AG

F

$A G$ AU

MN

MN

$A U$ AG

AU

$A U A G \quad C U$ PB ZN

SN

SN

SB

SN

AG

SN

AG AU

FE

CU

CU AU PB AG

$S B$

PB

$S B$

$A U A G$

AU

$P B$
$A G G$
N25 01

N24 33

N24 40

N25 38

N24 40

N24 40

N24 43

N25 19

N25 39

N25 25

N25 51

N24 55

N25 05

N23 59

N24 53

N24 34

N24 42

N24 31

N24 45

N25 15

N25 12

$\begin{array}{ll}N 19 & 07\end{array}$

$\begin{array}{ll}N 18 & 30 \\ N 19 & 03\end{array}$

$\begin{array}{ll}\mathrm{N} 19 & \mathrm{O} \\ \mathrm{N} 18 & 36\end{array}$

N18 36

$\begin{array}{ll}\text { N2O } & 58 \\ \text { N21 } & 27\end{array}$

N21 14

N21 22

N21 09

N2 409

N21 35

N2 105

N2 102

$\begin{array}{ll}\text { N2 } 1 & 07\end{array}$

N21 14

N2 125

N2O 54

$\begin{array}{ll}\text { N2O } & 54 \\ \text { N2 } 1 & 14\end{array}$

21

$\begin{array}{ll}\text { N21 } & 28 \\ \text { N2 } 1 & 22\end{array}$

N21 22

N2O 56

N21 32

N21 15

N21 27

N21 100

N21 10

N21 24

N17 04

N17 58

N17 40

N17 32

N17 34

$\mathrm{N} 1738$

N18 10

$\begin{array}{ll}\text { N18 } & 10 \\ \text { N17 } & 50 \\ N & \end{array}$

N17 50
W104 25

10600

$W 10410$
$W 103$

$103 \quad 14$

W103 14

10655

W102 50

W105 37

W105 42

w104 4

W104 36

$W 10637$
$W 10527$

$\begin{array}{lll}W 104 & 12\end{array}$

10240

W 10702

W105 28

$\begin{array}{ll}W 105 & 07\end{array}$

W106 18

W106 10

$\begin{array}{ll}W 104 & 14\end{array}$

W103 31

$\begin{array}{ll}W 103 & 31 \\ W 107 & 06\end{array}$

10706

$\begin{array}{ll}W 105 & 02 \\ W & \end{array}$

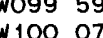

W100 07

W100 05

$\begin{array}{lll}W 100 & 13 \\ w 100 & 57\end{array}$

W100 57

W101 05

WO99 52

W101 05

W100 05

W 10043

$W 10008$

$W 10014$

W101 14

$W 10129$

W101 42

W101 17

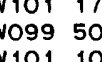

101 10

$W 10030$

W100 54

W101 29

W100 27

W101 29

$W 10104$

$W 100 \quad 09$

10020

W $100 \quad 15$

$W 10118$

WO99 55

WO99 47

W098 57

W 10001

W099 51

W099 50

W099 50

W100 10

wo99 40
8B7C7A7

4C45B7A7

4D7B7A7

4014A7A7

1A 147B3A7

4D 4C7A 7

8B7C7A7

8A47C7A7

4D 1C7A7

8B 1C5A7

4C 145A7A7

8B5C7A7

4D $1685 A 7$

4C7C7A 7

4D7C7A7

10A7C7A7

4C7B7A7

1B7C 1OA7

8B7C7A 7

3C 4C7A 7

4C7C7A7

4A $17 C 7 A 7$

4OA7C7A7

7D7B7A7

4D4C7A7

8B7C7A7

4C

8ATCTAT

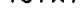

1B7C7A7

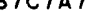

4C 17A7A7

10A7C7A7

8A7C7A7

4C7C7A7

1B7B7A7

4D7C7A7

IBTC7A7

IBTC7AT

BBTC7A7

BB7CTAT

1B7C7A7

1B7C7A7

4D47B7A7

1B7C7A7

8B7C7A 7

4C 1C7A 7

2A4C5A 7

3А4C $3 A 7$

3E 15C7A7

8B 15C7A7

$7 A 7 C 3 A$

$8 B 4 C 5 A 7$

7D5B $11 A 5$

4C 14B3A7

4C 14B3A7
$2 A 1 C 3 A 7$ 


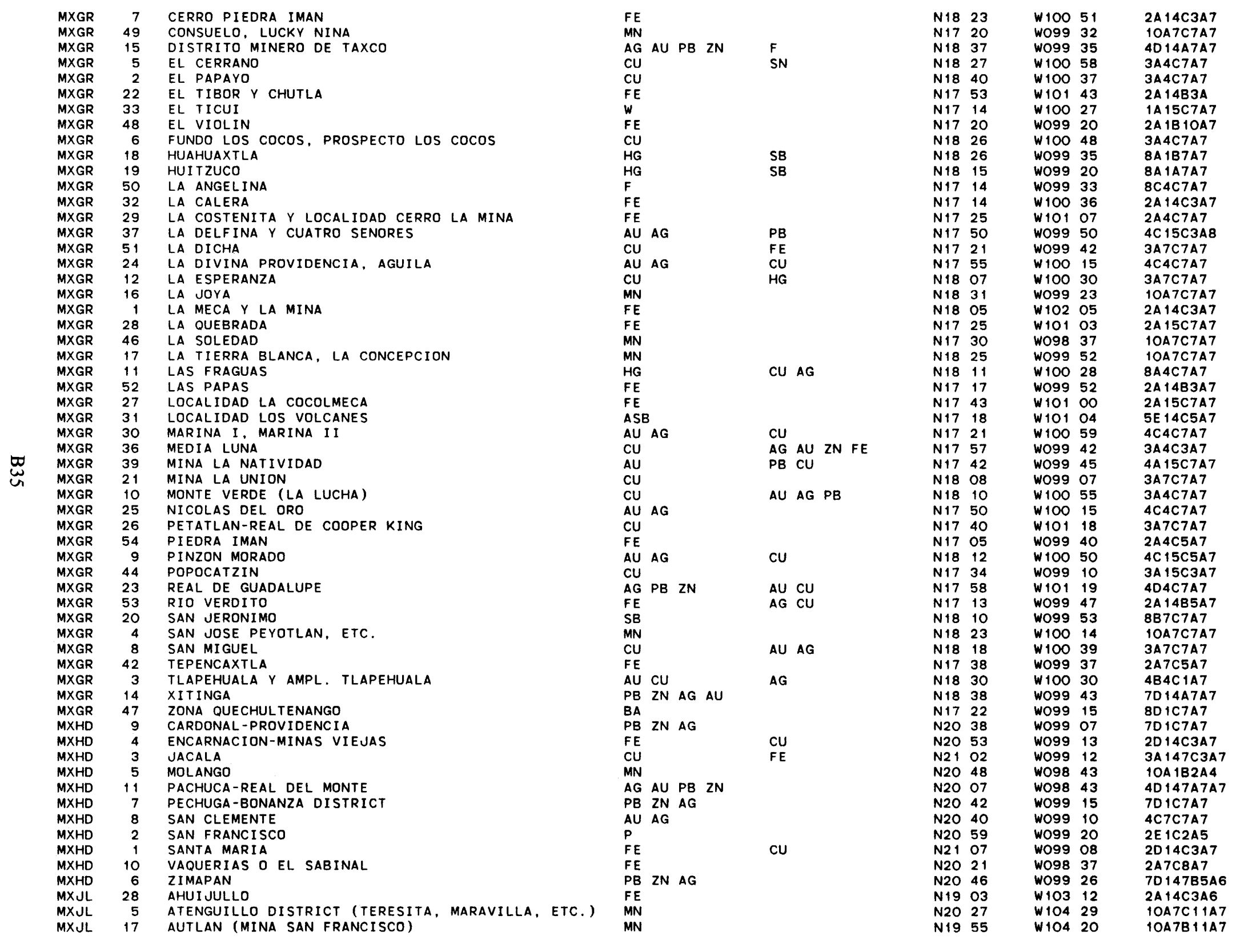




\begin{tabular}{|c|c|c|}
\hline MXJL & 7 & AYUTLA \\
\hline MXJL & 9 & CERRO DE PACHONA O LAS MINITAS \\
\hline MXJL & 20 & CIHUATLAN \\
\hline MXJL. & 10 & CINCO MINAS \\
\hline MXJL & 4 & CUALE-AMALTEA O DESMORONADO DISTRICT \\
\hline MXJL & 18 & CUTZMALA \\
\hline MXJL & 21 & EL GAVILAN, LA MARIPOSA, ET AL \\
\hline MXJL & 1 & 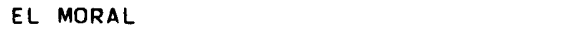 \\
\hline MXJL & 15 & EL ROBLE \\
\hline MXJL & 11 & EL SALTO-ACASICO \\
\hline MXUL & 2 & ETZATLAN DISTRICT \\
\hline MXJL & 27 & LA AMARILLA, SIEMPRE VIVA ET AL \\
\hline MXJL & 19 & LA HUERTA \\
\hline$M \times U L$ & 14 & LA MORA, LA REYNA, TACOTES \\
\hline MXJL & 6 & LA PROVIDENCIA, LA ESPERANZA \\
\hline MXUL & 20 & MARIA ELISA \\
\hline MXJL & 3 & MASCOTA \\
\hline MXUL & 22 & MATACRISTOS \\
\hline MXJL & 12 & MEZCALA ET AL \\
\hline MXUL & 8 & MINA LAS HUERTITAS \\
\hline MXUL & 23 & QUITIUPAN \\
\hline MXUL & 13 & ZONA CASPILOYA-EL PANTEON \\
\hline$M \times J L$ & 24 & ZONA PIHUMO (EL ENCINO ET AL) \\
\hline MXJL & 25 & ZONA SIERRA DEL ALO \\
\hline MXMC & 11 & AGUILILLA \\
\hline MXMC & 27 & ANGANGUEO \\
\hline MXMC & 12 & AQUILA Y ESTANZUELA \\
\hline MXMC & 48 & BASTON DEL COBRE \\
\hline MXMC & 35 & BENITO JUAREZ \\
\hline$M \times M C$ & 34 & CALTZONTZIN \\
\hline MXMC & 15 & CERRO CANTADOR \\
\hline MXMC & 28 & CIUDAD HIDALGO \\
\hline MXMC & 9 & COALCOMAN \\
\hline MXMC & 46 & COLMILLUDA $Y$ OTRAS MINAS \\
\hline MXMC & 32 & EL CARACOL \\
\hline MXMC & 31 & EL CARMEN \\
\hline MXMC & 2 & EL LIMON, JACONA \\
\hline MXMC & 10 & EL MARQUEZ, CANITAS \\
\hline MXMC & 36 & EL OLIVO \\
\hline MXMC & 39 & EL REALITO DE CHIRANGANGUEO \\
\hline MXMC & 18 & EL VENADO \\
\hline MXMC & 14 & EL VOLANTIN \\
\hline MXMC & 33 & ETUCUARO \\
\hline MXMC & 49 & HUETAMO \\
\hline MXMC & 42 & INGUARAN \\
\hline MXMC & 17 & JOVERO (VARIAS MINAS) \\
\hline MXMC & 16 & LA BUFAS (VARIAS MINAS) \\
\hline MXMC & 6 & LA CUCHILLA \\
\hline MXMC & 13 & LA GUAYABERA \\
\hline MXMC & 30 & LA REYNA \\
\hline MXMC & 3 & LA VERDE \\
\hline MXMC & 38 & LA YERBA BUENA \\
\hline MXMC & 8 & LAS CUESTA \\
\hline MXMC & 41 & LAS MOJARRAS AREA \\
\hline MXMC & 37 & LAS TRANCAS \\
\hline MXMC & 7 & LAS TROJES \\
\hline MXMC & 24 & LAS TRUCHAS \\
\hline$M \times M C$ & 25 & LOS CABIRES \\
\hline MXMC & 22 & LOS POZOS \\
\hline$M \times M C$ & 44 & MANGA DE CUIMBO \\
\hline
\end{tabular}

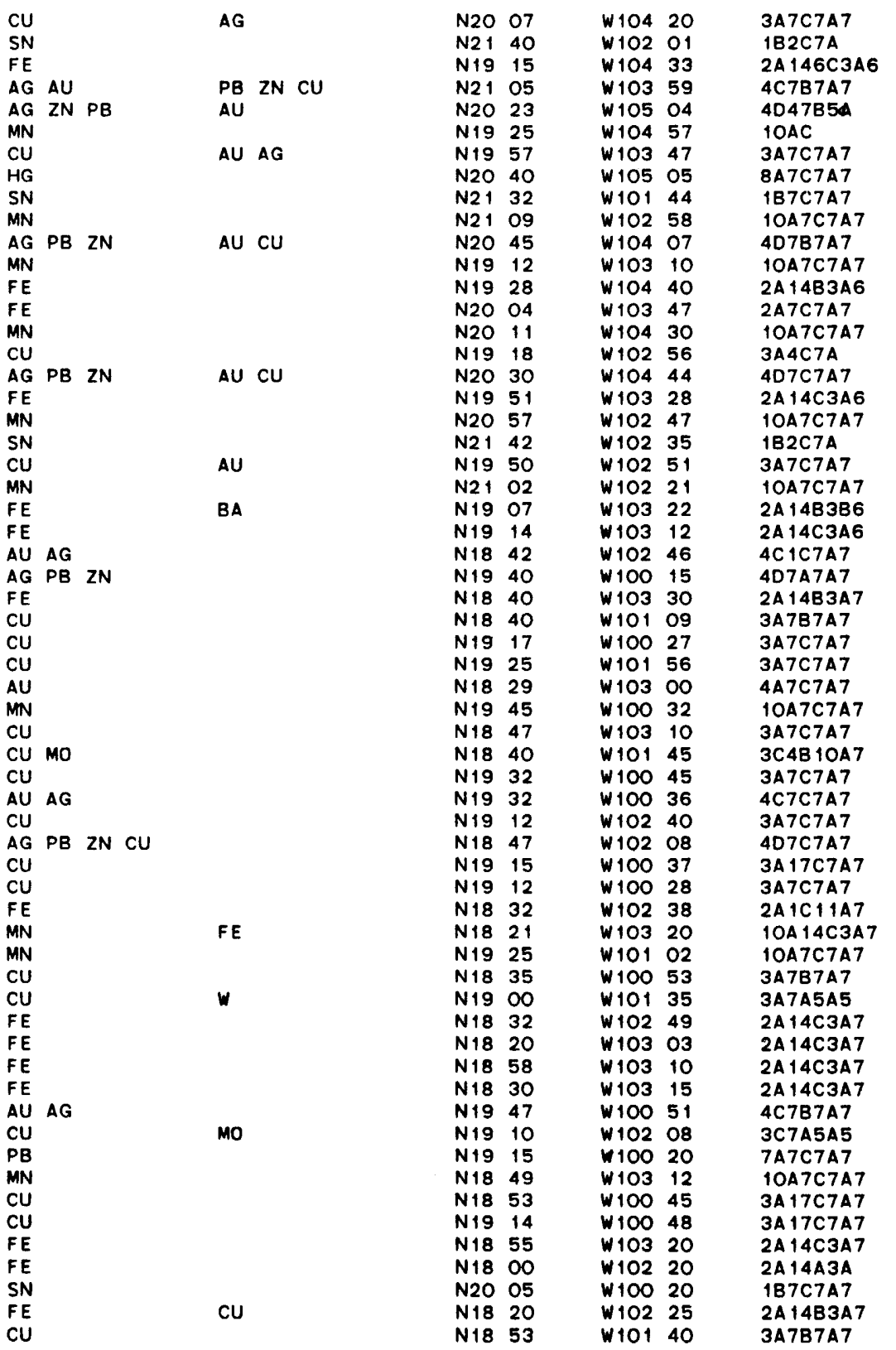




\begin{tabular}{|c|c|c|}
\hline MXMC & 5 & MINA EL TERRERO \\
\hline MXMC & 4 & MINAS LA TRINIDAD \\
\hline MXMC & 45 & OROPEO \\
\hline MXMC & 40 & PAPATZINGAN \\
\hline MXMC & 47 & REGION DE CHURUMUCO \\
\hline MXMC & 23 & RINCON DE VARILLO \\
\hline MXMC & 43 & SAN ISIDRO \\
\hline MXMC & 21 & SAN JOSE \\
\hline MXMC & 50 & SAN LUCAS \\
\hline MXMC & 26 & TLALPUJAHUA-EL ORO \\
\hline MXMC & 19 & TUMB I SCATIO \\
\hline MXMC & 20 & YACIMIENTO \\
\hline MXMC & 29 & ZINAPECUARO \\
\hline MXMC & 1 & ZONA DE TEPOLCATEPEC \\
\hline MXNA & 15 & AGUA CALIENTE \\
\hline MXNA & 24 & CACALUTAN \\
\hline MXNA & 23 & COMPOSTELA \\
\hline MXNA & 10 & COYOTES \\
\hline MXNA & 30 & EL DORADO \\
\hline MXNA & 22 & EL FAISAN, LA CORONILLA \\
\hline MXNA & 29 & EL PITON \\
\hline MXNA & 4 & EL TIGRE \\
\hline MXNA & 1 & GUAMUCHIL \\
\hline MXNA & 14 & HUAJIMIC \\
\hline MXNA & 13 & HUAYNAMOTA GROUP, OCOTAN \\
\hline MXNA & 9 & LA CHIRIPA Y DORADITO \\
\hline MXNA & 27 & LA ESPERANZA \\
\hline MXNA & 25 & LA PURISIMA, LA VIRGEN \\
\hline MXNA & 21 & LA YESCA \\
\hline MXNA & 6 & LAS CRUCES \\
\hline MXNA & 5 & LOS ESPEJOS \\
\hline MXNA & 8 & LOS NOPALITOS \\
\hline MXNA & 19 & MALINAL \\
\hline MXNA & 2 & MINA CUCHARAS \\
\hline MXNA & 11 & P. V. 4 (EL ZOPILOTE) \\
\hline MXNA & 3 & PUYEQUE \\
\hline MXNA & 16 & SAN FRANCISCO \\
\hline MXNA & 7 & SAN FRANCISCO, EL CARMEN, ETC. \\
\hline MXNA & 28 & SANTA EDMIGE \\
\hline MXNA & 20 & SANTA MARIA DEL ORO \\
\hline MXNA & 12 & SANTIAGO IXCUINTLA \\
\hline MXNA & 17 & ZONA DE ACUILAPILCO \\
\hline MXNA & 18 & ZONA DE COAPILLA \\
\hline MXNL & 14 & CHAPOPOTE 1 Y 2 \\
\hline MXNL & 1 & DOLORES \\
\hline MXNL & 18 & DULCES NOMBRES \\
\hline MXNL & 13 & EL CANARIO \\
\hline MXNL & 3 & GOLONDRINAS O CARRIZAL \\
\hline MXNL & 5 & LA PENA \\
\hline MXNL & 16 & LA VICTORIA \\
\hline MXNL & 4 & MERCEDES Y HERMINIA (SABINAS HGO.) \\
\hline MXNL & 2 & MINERAL DE SAN ANTONIO \\
\hline MXNL & 10 & MITRA Y ARTEAGA \\
\hline MXNL & 8 & MONSERRAT, VALLE ALTO \\
\hline MXNL & 17 & P.V. 15-EL RUCIO \\
\hline MXNL & 9 & RICONADA \\
\hline MXNL & 7 & SANTA CATARINA \\
\hline MXNL & 6 & SIERRA DEL FRAILE \\
\hline MXNL & 15 & ZONA DE ARAMBERRI \\
\hline MXNL & 11 & ZONA SAN LUCAS \\
\hline
\end{tabular}

\begin{tabular}{|c|c|c|c|c|c|c|c|c|}
\hline $\mathrm{cu}$ & & & & N18 & 56 & $W 102$ & 35 & $3 A 4 C 7 A 7$ \\
\hline PB & $\mathrm{ZN}$ & $\mathrm{CU}$ & & N19 & 01 & $W 102$ & 21 & 7D4C7A7 \\
\hline $\mathrm{cu}$ & & & & N18 & 50 & $W 101$ & 50 & ЗАТC:7A7 \\
\hline $\mathrm{CU}$ & & & & N19 & 03 & $W 100$ & 47 & 3A17C7A7 \\
\hline $\mathrm{cU}$ & & & & N18 & 38 & W 101 & 38 & 3А7C7A7 \\
\hline cu & $A U$ & & & N18 & 15 & $W 102$ & 07 & 3E $1 C 7 A 7$ \\
\hline CU & & & & N18 & 57 & W101 & 55 & 3А7C5A5 \\
\hline $\mathrm{cu}$ & & & & N18 & 20 & $W 102$ & 13 & 3А4C7A7 \\
\hline SB & & & & N18 & 35 & $W 100$ & 48 & $8 B 7 C 7 A 7$ \\
\hline AU & $A G$ & & & N19 & 50 & $W 100$ & 10 & 4C 17A7A7 \\
\hline$A U$ & $A G$ & & $\mathrm{CU}$ & N18 & 30 & $W 102$ & 20 & $4 B 4 C 7 A 7$ \\
\hline FE & & & & $N 18$ & 30 & W 102 & 10 & $2 A 1 C 3 A 7$ \\
\hline $\mathrm{PB}$ & $A G$ & $Z N$ & & N19 & 52 & $w 100$ & 50 & 7D7C7A7 \\
\hline BA & & & & N19 & 11 & $W 102$ & 50 & $8 D 7 C 7 A 7$ \\
\hline AU & $A G$ & & & N21 & 40 & W 104 & 33 & 4C7C7A7 \\
\hline AU & $A G$ & & & N21 & 13 & $W 104$ & 18 & 4C7C7A7 \\
\hline AU & $A G$ & & & N21 & 12 & $W 104$ & 55 & 4C7C7A7 \\
\hline AU & $A G$ & & & N22 & 07 & W105 & 10 & 4C7C7A7 \\
\hline AU & $A G$ & & & $\mathrm{~N} 2 \mathrm{O}$ & 51 & $W 104$ & 26 & 4C4C7A \\
\hline AU & $A G$ & & & N21 & 15 & W 105 & 03 & $4 C 47 C 7 A$ \\
\hline$A U$ & & & & N2O & 55 & W 104 & 30 & $\triangle A 7 C 7 A 7$ \\
\hline$A U$ & $\mathrm{CU}$ & & & N22 & 29 & W 105 & 24 & 4B7C7A7 \\
\hline AU & AG & & & N22 & 58 & W105 & 10 & 4С7C7A7 \\
\hline SN & & & & N21 & 40 & W 104 & 20 & $1 B 7 C 7 A 7$ \\
\hline$A G$ & PB & $Z N$ & $\triangle U C U$ & N2 1 & 50 & $W 104$ & 30 & 4D7C7A7 \\
\hline$A U$ & $A G$ & & & N22 & 12 & W 105 & 15 & $4 C 4 C 7 A 7$ \\
\hline $\mathrm{cu}$ & & & & N21 & 02 & W104 & 34 & $3 A 4 C 7 A$ \\
\hline AU & $A G$ & & & N2O & 58 & W105 & 00 & 4C 4C7A \\
\hline $\mathrm{CU}$ & & & $A G$ & N21 & 16 & $W 104$ & 01 & 3А7C7A7 \\
\hline SN & & & & N22 & 33 & W 104 & 45 & 1B7C7A7 \\
\hline SN & & & & N22 & 39 & $W 104$ & 48 & 1B7C7A7 \\
\hline SN & & & & N22 & 20 & W 104 & 41 & 1B7B7A7 \\
\hline AU & & & & N21 & 20 & W105 & 05 & 4A7C7A7 \\
\hline $\mathrm{cu}$ & & & $A U$ & N22 & 52 & W105 & 15 & 3E 7C7A 7 \\
\hline$A U$ & $A G$ & & & N21 & 57 & W 104 & 56 & 4C7C7A7 \\
\hline AU & AG & & & N22 & 35 & W105 & 23 & 4C7C7A7 \\
\hline FE & & & & N2 1 & 25 & W 104 & 55 & 2A7C7A7 \\
\hline$A U$ & $A G$ & & & N22 & 26 & W 105 & 10 & 4C7C7A7 \\
\hline $\mathrm{cu}$ & $A U$ & & & $\mathrm{~N} 2 \mathrm{O}$ & 58 & W 104 & 25 & $3 E 4 C 7 A$ \\
\hline $\mathbf{A G}$ & $A U$ & PB ZN & & N2 1 & 17 & $W 104$ & 35 & 4D4C7A \\
\hline$A U$ & & & $\mathrm{CU}$ & N21 & 48 & W105 & 10 & АA7B7A7 \\
\hline$A U$ & AG & & & N21 & 26 & $W 104$ & 31 & 4C7C7A7 \\
\hline $\mathrm{AU}$ & AG & & & N21 & 22 & W104 & 45 & 4C47C7A \\
\hline $\mathbf{P}$ & & & & N25 & 42 & W099 & 44 & 2E 1C2A5 \\
\hline $\mathrm{cu}$ & & & & N27 & 03 & $W 100$ & 30 & 3А $1 C 7 A 7$ \\
\hline AG & PB & $2 \mathrm{~N}$ & & N23 & 48 & W099 & 35 & 4D 1C7A7 \\
\hline $\mathrm{FE}$ & & & $\mathbf{P}$ & N24 & 01 & $W 100$ & 12 & $2 A+C 7 A 7$ \\
\hline FE & & & & N26 & 44 & $W 100$ & 30 & 2A 14C3A7 \\
\hline$M N$ & & & & N26 & 03 & $w 100$ & 33 & 1OA 1C7A7 \\
\hline MN & & & & N24 & $\infty$ & W099 & 46 & 1OA IC7A7 \\
\hline $\mathbf{P}$ & & & & N26 & 32 & $W 100$ & 14 & $2 E 1 C 7 A 7$ \\
\hline$A G$ & PB & $2 \mathrm{~N}$ & & N26 & 57 & $w 100$ & 05 & 4D 14C3А 7 \\
\hline $\mathbf{P}$ & & & & N24 & 50 & $W 100$ & 48 & 2E 1B2A4 \\
\hline$A G$ & PB & $2 \mathrm{~N}$ & & N25 & 36 & $W 100$ & 18 & $401 C 7 A 7$ \\
\hline PB & $\mathrm{ZN}$ & & & N23 & 56 & $w 100$ & 31 & 7C 1C7A7 \\
\hline $\mathrm{FE}$ & & & & N25 & 27 & W100 & 08 & 2A14C3A7 \\
\hline MN & & & & N25 & 43 & $W 100$ & 27 & 1OA 1C7A7 \\
\hline GYP & & & & N25 & 54 & $W 100$ & 34 & 6A1C6A4 \\
\hline$B A$ & & & & N24 & 05 & W099 & 48 & $8 D\{B 7 A 7$ \\
\hline BA & & & SR & N24 & 56 & $W 100$ & 12 & $8 D 1 C 7 A 7$ \\
\hline
\end{tabular}




\begin{tabular}{|c|c|c|}
\hline MXNL & 12 & ZONA SAN MARCOS-SANTA CLARA \\
\hline $\operatorname{MxOX}$ & 35 & ALOAPAN \\
\hline MXOX & 5 & AMATITLAN \\
\hline MXOX & 23 & APOALA \\
\hline MXOX & 18 & CACALOTEPEC \\
\hline Mxox & 20 & CAMATLAN Y HUEJOTITLAN \\
\hline $\operatorname{MxOX}$ & 85 & CERRO PENUELAS Y POZO TORIBIO \\
\hline $\operatorname{MxOX}$ & 13 & CHAYUCO O LA FIERROSA \\
\hline $\operatorname{MxOX}$ & 16 & CONCEPCION PAPALOS \\
\hline $\operatorname{MXOX}$ & 75 & COSTACHE \\
\hline $\operatorname{MxOX}$ & 26 & COYOTEPEC, LLANO PERALES \\
\hline $\operatorname{MxOX}$ & 51 & CUILAPAN \\
\hline $\operatorname{MxOX}$ & 22 & DUAYACO \\
\hline $\operatorname{MxOX}$ & 98 & ECATEPEC \\
\hline MxOX & 81 & EUUTLA \\
\hline MXOX & 60 & EL AGUACATE \\
\hline MXOX & 59 & EL CARNERO \\
\hline $\operatorname{MxOX}$ & 94 & EL MARMOL \\
\hline $\operatorname{MxOX}$ & 90 & EL MERODIO, EL EUSTAQUIO, LA VENTOSA \\
\hline $\operatorname{MxOX}$ & 8 & EL PIRATA \\
\hline $\operatorname{MxOX}$ & 62 & EL RECIBIMIENTO \\
\hline Mxox & 49 & ESTETLA \\
\hline $\operatorname{MxOX}$ & 64 & HACIENDA VIEUA \\
\hline $\operatorname{MxOX}$ & 37 & IXTLAN DE JUAREZ \\
\hline $\operatorname{MxOX}$ & 24 & JALTEPETONGO \\
\hline MxOX & 82 & JAYACAXTEPEC \\
\hline MXOX & 80 & JUQUILA \\
\hline $\operatorname{MxOX}$ & 105 & LA CANDELARIA \\
\hline $\operatorname{MxOX}$ & 30 & LA CARBONERA \\
\hline MxOX & 32 & LA JOYA \\
\hline MXOX & 72 & LA SOLEDAD \\
\hline $\operatorname{mxOX}$ & 74 & LACHIADOVA \\
\hline $\operatorname{MxOX}$ & 110 & LAS ESPERANZAS \\
\hline MXOX & 40 & LAXOPA \\
\hline MxOX & 43 & LLANO DE SAN VICENTE \\
\hline $\operatorname{mxOx}$ & 92 & LOAYAGA \\
\hline MXOX & 29 & MACUILTIANGUIS \\
\hline $\operatorname{MXOX}$ & 86 & MATIAS ROMERO \\
\hline MXOX & 4 & MILTEPEC \\
\hline MXOX & 9 & MINA LA FORTUNA \\
\hline MXOX & 10 & MINA LA SOLEDAD \\
\hline $\operatorname{MxOX}$ & 103 & MINAS GUADALUPE Y ZONA DE COBAS \\
\hline $\operatorname{mxOX}$ & 68 & MIXTEPEC \\
\hline MXOX & 95 & MIXTEOUILLA \\
\hline MXOX & 50 & MUNANA \\
\hline $\operatorname{MxOX}$ & 91 & NILTEPEC \\
\hline MXOX & 84 & NIZADUGA \\
\hline Mxox & 101 & PASO NAVEGANTE \\
\hline MxOX & 104 & PIEDRAS BLANCAS \\
\hline MXOX & 107 & PINAS \\
\hline MXOX & 66 & PLAN MINA \\
\hline $\operatorname{MXOX}$ & 111 & PLAYAS \\
\hline $\operatorname{MxOX}$ & 108 & PLUMA HIDALGO \\
\hline $\operatorname{MxOX}$ & 69 & POBLETE \\
\hline $\operatorname{MxOX}$ & 109 & POCHUTLA \\
\hline MXOX & 44 & PURIFICACION \\
\hline MxOX & 73 & QUECHAPA \\
\hline MXOX & 57 & QUIATONI \\
\hline MXOX & 27 & QUIOTEPEC \\
\hline MXOX & 67 & SAACHILA (TRINIDAD) \\
\hline
\end{tabular}

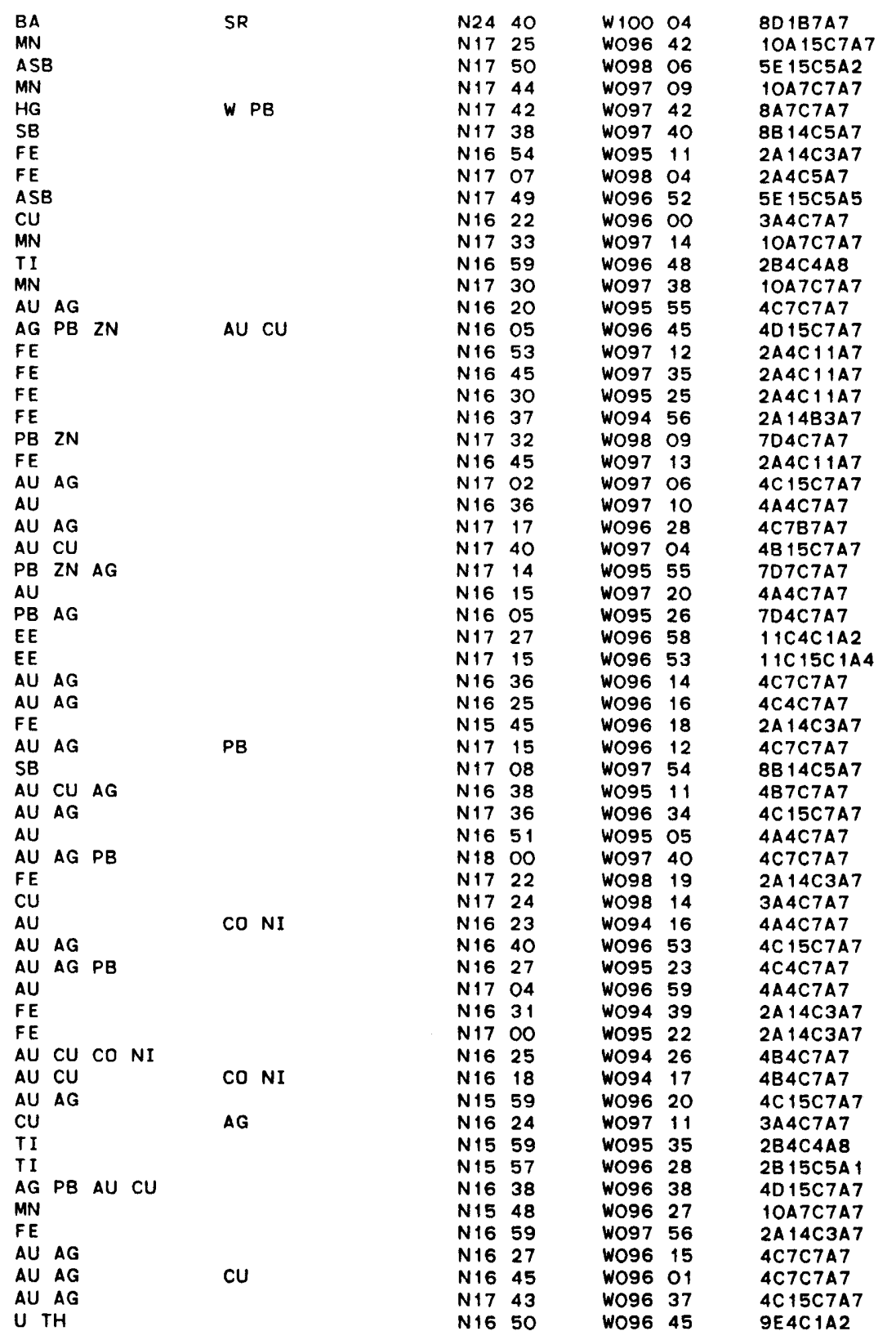




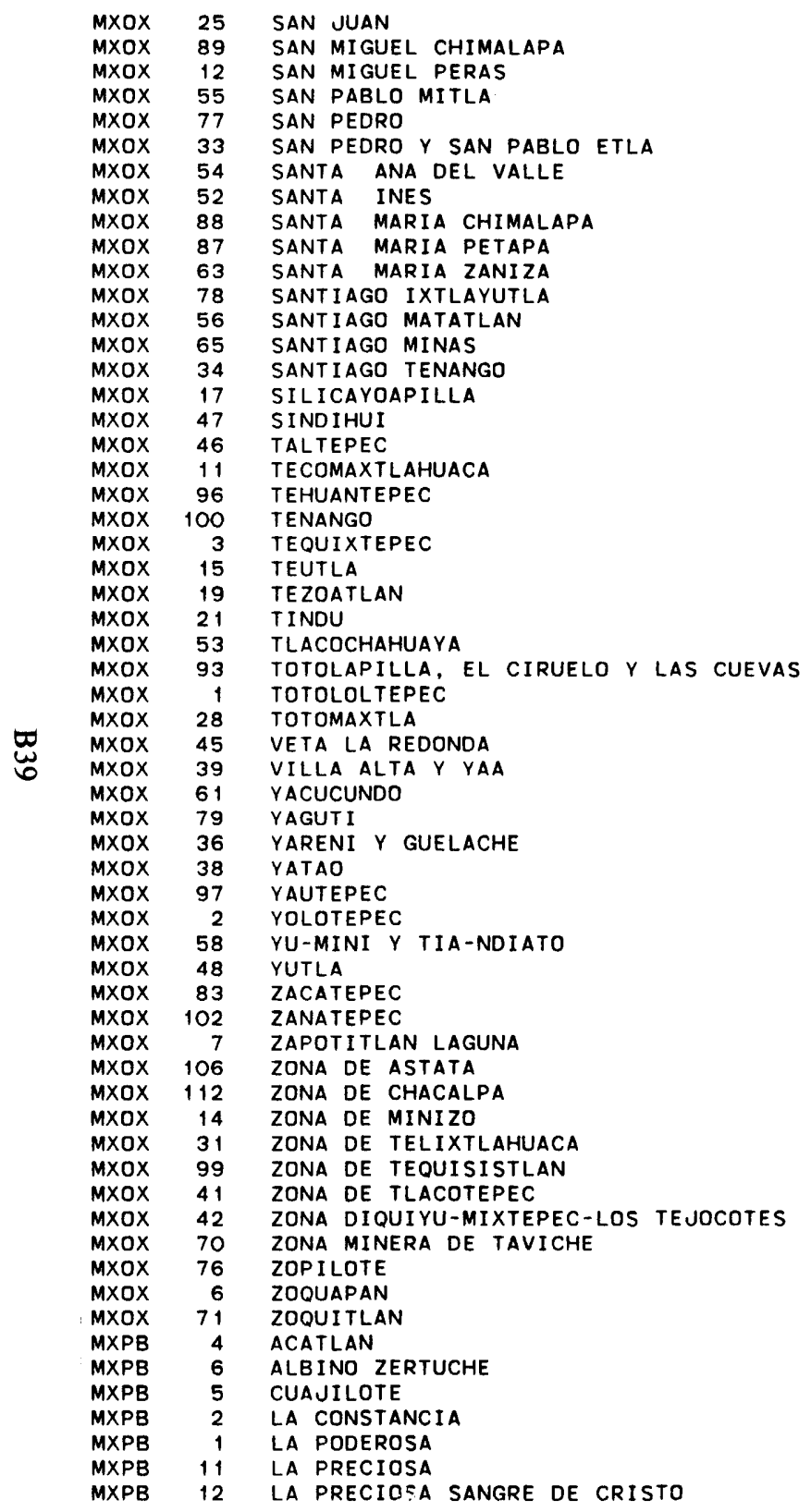

AU AG

$A U A G C U$

$A G \quad P B \quad Z N$

AU

I I

$A U$ AG

TI

AU AG

FE

AU CU

PB ZN CU AU

$A U$

AU

AU

$A U A G$

MN

$A G P B C U A U$

AU

MN

PB AG

FE

PB ZN

PB AG

$\begin{array}{ll}A G & P B \\ P B & 2 N\end{array}$

CU

$A U A G$

$A U A G$

$A U$ AG

AU

PB ZN AG

$A U$ AG

MN

FE

TI

GRF

$A U A G$

$A G \quad P B \quad Z N \quad A U$

SB

$A U$ AG PB ZN

$\checkmark$ TH

PB $A G \quad C U$

SB

MN

SB
MN

MN

MN

MN
N17 40

N16 43

N17 15

$\begin{array}{ll}N 16 & 55 \\ N 16 & 19\end{array}$

N17 15

N16 58

N16 55

$\begin{array}{ll}N 16 & 54 \\ N 16 & 48\end{array}$

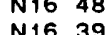

$\begin{array}{ll}N 16 & 39 \\ N 16 & 30\end{array}$

N16 50

N16 30

N17 27

$\mathrm{N} 1750$

$\mathrm{N} 1700$

N17 08

$\begin{array}{ll}N 17 & 20 \\ N 16 & 27\end{array}$

$\begin{array}{ll}N 16 & 27 \\ N 16 & 17\end{array}$

N18 05

N17 58

N17 42

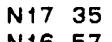

N16 57

N16 32

N18 15

$\begin{array}{ll}N 17 & 40 \\ N 17 & 16\end{array}$

N17 19

N17 19

N16 50

$\begin{array}{ll}N 16 & 15 \\ N 17 & 17\end{array}$

N17 17

N17 21

N16 26

$\begin{array}{ll}N 18 & 07\end{array}$

N16 51

N17 04

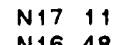

$\begin{array}{ll}N 16 & 48 \\ N 17 & 38\end{array}$

N16 01

N15 54

N16 08

N17 20

$\begin{array}{ll}N 16 & 24 \\ N 17 & 25\end{array}$

$\begin{array}{ll}N 17 & 25 \\ N 17 & 17\end{array}$

N17 17

$\begin{array}{ll}N 16 & 39\end{array}$

N16 24

N17 43

N 1633

N18 12

N18 O5

N18 03

N18 27

N2O 07

N19 15

N19 05
W096 55

W98 17

W096 20

W096 42

W096 50

W096 23

09652

0964

W094 42

W95 08

W097 42

W99 42

W096 15

WO97 12

W096 47

W097 44

W097 22

W097 22

W098 02

W095 19

09535

W097 40

WO96 42

W097 50

W097 53

28

W096 28

109537
109745

W097 45

W096 34

W097 34

W096 07

W097 10

W97 40

109639

W096 16

W095 59

W097 45

W097 32

W097 09

W095 50

WO94 20

W098 24

WO95 40

W095 55

W098 01

W096 54

WO95 39

W097 54

W997 55

W096 32

09639

W098 18

V096 24

W098 00

W098 30

W098 03

W098 03

W098 03

W097 10

W097 23
$4 C 15 C 7 A 7$

4C7CTA7

AD7C7A7

4A4C7A7

2B 15C 1A4

4C 15C7A7

2B4C5A2

4C7C7A7

2A 4B3A7

2A 4C3A7

7D7C7A7

4A 4C7A7

4C 15C7A7

8B 14C5A7

4C4C7A7

4C7C7A7

10A7C7A7

3B7C7A7

4D 15C7A7

10A7C7A7

10A7C7A7

7D7C7A7

$2 A 14 C 3 A 7$

2A 14C3A7

7D15C7A7

7015C7A

4D46C7A7

704C7A7

3A 4C7A7

4C 15C7A7

7A7C7A7

4C 15C7A7

4A37C4AB

3A 15C7A7

7D 15C7A7

4C4C7A7

2A 14C3A7

2B4B5A7

2B4C $4 A 8$

11E 15C 1OA

4C4C7A7

4D7C7A7

8B 1485A7

4C7C7A7

9E4C $1 A 2$

7D7C7A7

8B 14C 10A7

10A7C7A7

8B7C7A7

10A 15C7A7

10A7C7A7

10A7C7A7

10A7C7A7

10A7C7A 7 


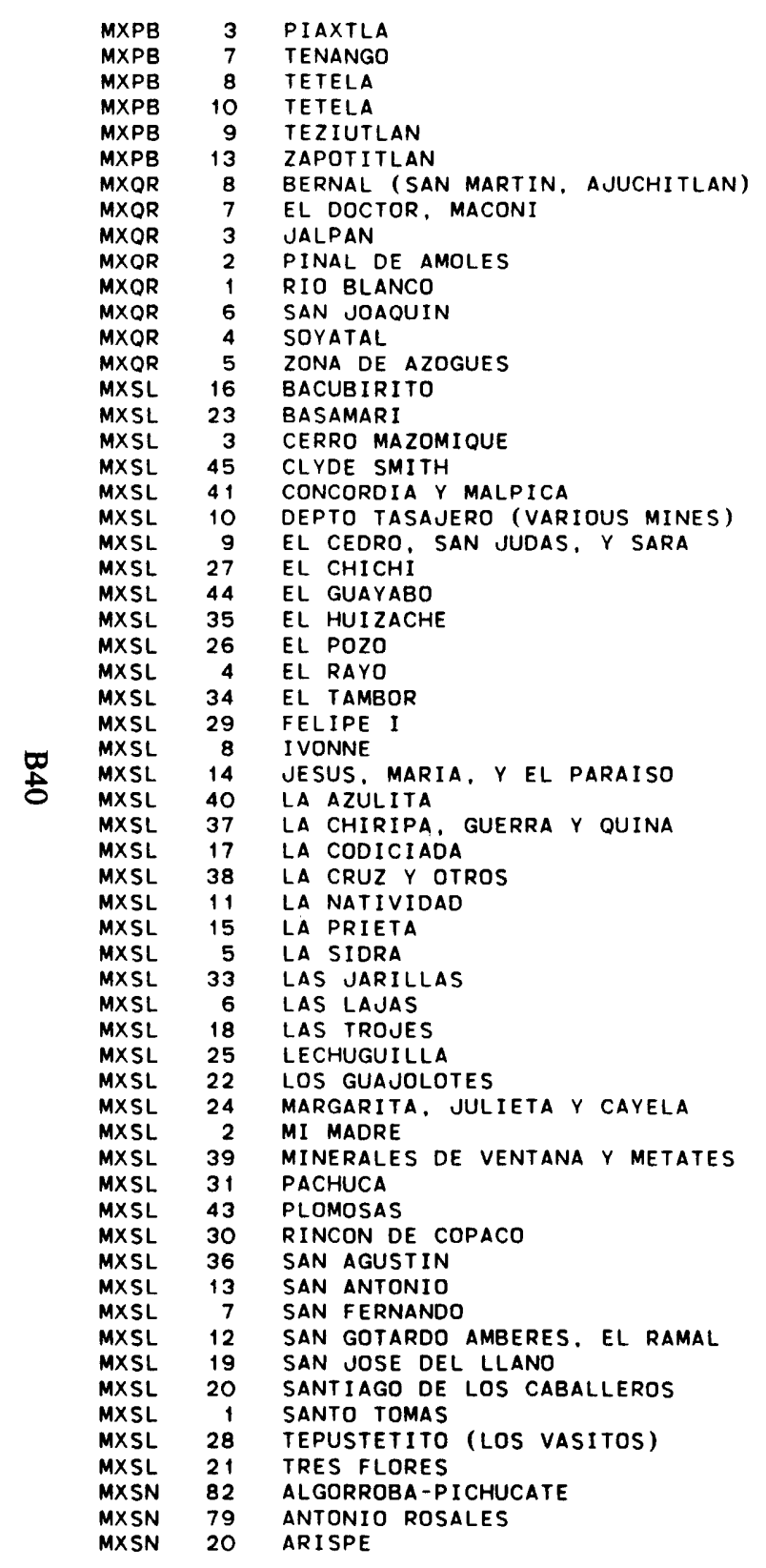

\begin{tabular}{|c|c|c|c|c|c|c|c|c|c|}
\hline & N18 & 19 & W098 & 21 & 10A7C7A7 \\
\hline \multicolumn{5}{|c|}{ MN } & $N 19$ & 55 & W097 & 55 & 10A7C7A7 \\
\hline \multicolumn{5}{|l|}{$A U$} & N19 & 54 & W097 & 43 & $4 A 7 C 7 A 7$ \\
\hline \multicolumn{5}{|c|}{ MN } & $N 19$ & 48 & W097 & 48 & 1OA7C7A7 \\
\hline \multicolumn{5}{|c|}{ мO } & N19 & 50 & W097 & 20 & $3 C 1 C 7 A 7$ \\
\hline \multicolumn{5}{|c|}{ SB } & N18 & 15 & W097 & 30 & $8 B 7 C 7 A 7$ \\
\hline \multicolumn{5}{|c|}{$A U A G$} & $\mathrm{~N} 2 \mathrm{O}$ & 53 & $w 100$ & $\infty 0$ & $4 C\{C 7 A 7$ \\
\hline PB & $Z N$ & $A G$ & $A U$ & $\mathrm{CU}$ & $\mathrm{N} 2 \mathrm{O}$ & 51 & W099 & 33 & $7 D 14 C 5 A 7$ \\
\hline$A U$ & $A G$ & PB ZN & $\mathrm{CU}$ & & N21 & 12 & W099 & 28 & $401 C 7 A 7$ \\
\hline PB & $2 N$ & $\Delta G$ & $\mathrm{CU}$ & & N21 & 09 & W099 & 38 & $701 C 7 A 7$ \\
\hline$A G$ & $\mathrm{AU}$ & & & & N21 & 12 & W099 & 45 & $4 C 5 C 7 A 7$ \\
\hline$H G$ & & & & & N2O & 55 & W099 & 33 & 8A7C7A7 \\
\hline SB & & & & & N21 & 00 & W099 & 43 & 8B 1B5A7 \\
\hline HG & & & & & N21 & 00 & W099 & 34 & 8A4C7A7 \\
\hline$A U$ & & & & & N25 & 51 & W 107 & 55 & $4 A 3 C 4 A 8$ \\
\hline$C U$ & & & & & N25 & 20 & W107 & 45 & $3 A 4 C 7 A 7$ \\
\hline $\mathrm{FE}$ & & & $\mathrm{CU}$ & $A U$ & N26 & 44 & W108 & 15 & 2A14C3A6 \\
\hline MN & & & & & N23 & $\infty$ & W105 & 48 & 1OA7C7A7 \\
\hline $\mathrm{cu}$ & & & $A U$ & AG MO & N23 & 16 & W106 & 07 & ЗА 4 СА \\
\hline PB & $\mathrm{ZN}$ & $\Delta G$ & & & N26 & 05 & W108 & 33 & 7D7C7A7 \\
\hline PB & $\mathrm{ZN}$ & $A G$ & & & N26 & 05 & $W 108$ & 25 & 4D 4C7A 7 \\
\hline$A G$ & PB & $A U$ & & & N24 & 48 & W 107 & 23 & 4D4C7A \\
\hline$w$ & MO & & & & N22 & 57 & W105 & 34 & 1A $14 B 7 A$ \\
\hline$A U$ & $A G$ & & & & N24 & 03 & W106 & 25 & 4C4C7A \\
\hline PB & $\mathrm{ZN}$ & AG & $A U$ & $\mathrm{CU}$ & N24 & 58 & W 107 & 13 & $4015 C 3 A$ \\
\hline$A U$ & $A G$ & & & & N26 & 30 & W108 & 10 & $4 C 4 C 7 A 7$ \\
\hline$A U$ & & & & & N24 & 08 & $W 106$ & 30 & $\triangle A 7 C 7 A 7$ \\
\hline FE & & & & & N24 & 37 & W 107 & 10 & $2 A+4 C 3 A 6$ \\
\hline $\mathrm{CU}$ & $A U$ & & PB & & N26 & 08 & W108 & 37 & 3E $15 C 7 A 7$ \\
\hline$A U$ & $\mathbf{A G}$ & & $\mathrm{cu}$ & & N26 & 08 & W107 & 58 & $4 C 47 C 7 A 7$ \\
\hline $\mathrm{CU}$ & & & & & N23 & 40 & W106 & 10 & 3A4C \\
\hline$A U$ & $A G$ & & & & N23 & 56 & W106 & 23 & $4 C 4 C 7 A$ \\
\hline NI & co & $\mathrm{CU}$ & & & N25 & 52 & W 107 & 37 & $508 \mathrm{C}$ \\
\hline$A U$ & $A G$ & & & & N23 & 50 & W106 & 20 & 4C $4 C 7 A$ \\
\hline $\mathrm{cu}$ & & & & & N26 & 02 & W108 & 39 & $3 A 6 C 7 A 7$ \\
\hline MN & & & & & N26 & $\infty 0$ & $W 108$ & $\infty$ & 1OA7C7A7 \\
\hline$A U$ & $A G$ & & & & N26 & 29 & W108 & 02 & $4 C 4 C 7 A 7$ \\
\hline$\Delta U$ & $A G$ & & & & N24 & 16 & W 106 & 32 & 4C7C7A7 \\
\hline $\mathrm{CU}$ & & & & & N26 & 28 & $W 108$ & 39 & 3A14C7A7 \\
\hline$A U$ & $A G$ & & & & N25 & 41 & $W 107$ & 24 & $4 C 4 C 7 A 7$ \\
\hline FE & $\mathrm{CU}$ & & & & N24 & 58 & W 107 & 19 & $2 D 14 C 3 A$ \\
\hline$C U$ & $A U$ & & & & N25 & 31 & $W 107$ & 29 & 3E $4 C 7 A 7$ \\
\hline CU & $w$ & & $A U$ & AG & N25 & 10 & W107 & 51 & ЗА 14C $3 A$ \\
\hline FE & $\mathrm{CU}$ & & & & N26 & 50 & W108 & 15 & $201 C 7 A 6$ \\
\hline$A U$ & $A G$ & & & & N23 & 48 & W106 & 00 & 4C7C7A7 \\
\hline$A U$ & $\Delta G$ & & & & N24 & 20 & $W 106$ & 42 & 4C $17 B 7 A 7$ \\
\hline PB & $A G$ & $A U$ & & & N23 & 05 & $W 105$ & 25 & $707 C 7 A 7$ \\
\hline $\mathrm{cu}$ & MO & & $\mathbf{A G}$ & $A U$ & N24 & 32 & W107 & 00 & $3 C 5 C 7 A$ \\
\hline$A U$ & $A G$ & & & & N23 & 58 & W106 & 32 & 4C7C7A7 \\
\hline$A U$ & $A G$ & & & & N26 & 03 & $W 108$ & 10 & $\triangle C 4 C 7 A 7$ \\
\hline $\mathrm{CU}$ & & & & & N26 & 22 & $W 108$ & 21 & 3A4C5A7 \\
\hline PB & $\mathrm{ZN}$ & & $A G$ & & N26 & 01 & $W 108$ & 30 & 7D4C7A7 \\
\hline $\mathrm{cu}$ & & & $A U$ & $A G$ & N25 & 37 & $W 107$ & 20 & ЗА $17 C 7 A 7$ \\
\hline PB & $\mathrm{ZN}$ & $\mathrm{CU}$ & & & N25 & 35 & W107 & 20 & 7D7C7A7 \\
\hline $\mathrm{CU}$ & & & FE & & N26 & 53 & $W 108$ & 08 & उA 14B3A6 \\
\hline FE & & & $\mathrm{CU}$ & & N24 & 40 & $W 107$ & 06 & $2 A 14 C 3 A 6$ \\
\hline $\begin{array}{l}\mathrm{CU} \\
\mathrm{AU}\end{array}$ & $\mathbf{A G}$ & $A U$ & & & $\begin{array}{l}\text { N25 } \\
\text { N26 }\end{array}$ & $\begin{array}{l}32 \\
56\end{array}$ & $\begin{array}{l}W 107 \\
W 108\end{array}$ & $\begin{array}{l}14 \\
31\end{array}$ & $\begin{array}{l}\text { 3E5C7A7 } \\
4 A 7 C 7 A 7\end{array}$ \\
\hline $\begin{array}{l}C U \\
S B\end{array}$ & PB & $2 N$ & $A G$ & $A U$ & $\begin{array}{l}\text { N26 } \\
\text { N3O }\end{array}$ & $\begin{array}{l}56 \\
20\end{array}$ & $\begin{array}{l}W 108 \\
W 110\end{array}$ & $\begin{array}{l}56 \\
07\end{array}$ & $\begin{array}{l}\text { 307C7A7 } \\
8 B 7 C 7 A 7\end{array}$ \\
\hline
\end{tabular}




\begin{tabular}{|c|c|c|}
\hline MXSN & 11 & ATIL \\
\hline MXSN & 1 & BAHIA DE ADAIR \\
\hline MXSN & 66 & BAROLLEGA \\
\hline MXSN & 31 & BAVIACORA (SAN ANTONIO Y OTROS) \\
\hline MXSN & 75 & BLANCA, URREA, LAS LOMAS \\
\hline MXSN & 8 & CABORCA \\
\hline MXSN & 54 & CAJON DE ONAPA \\
\hline MXSN & 12 & CANANEA \\
\hline MXSN & 21 & CARR \\
\hline MXSN & 50 & CIENAGUITA \\
\hline MXSN & 28 & CUMPAS \\
\hline MXSN & 52 & DOLORES \\
\hline MXSN & 27 & DOS CABEZAS \\
\hline MXSN & 19 & DOS NACIONES \\
\hline MXSN & $12 \mathrm{~A}$ & EL ALACRAN \\
\hline MXSN & 7 & EL ANTIMONIO \\
\hline MXSN & 38 & EL CHORRO \\
\hline MXSN & 81 & EL COBRE Y SAN BARTOLO \\
\hline MXSN & 68 & EL MANTO \\
\hline MXSN & 74 & EL TORO, NOCHE BUENA-VETA GRANDE \\
\hline MXSN & 76 & EL TRANVIA \\
\hline MXSN & 23 & ESQUEDA DISTRICT \\
\hline MXSN & $8 \mathrm{~A}$ & FORTUNA DEL COBRE \\
\hline MXSN & 53 & GALLO DE ORO \\
\hline MXSN & 13 & GAMER, SHANGRI LA, ET AL \\
\hline MXSN & 40 & HERMOS I LLO \\
\hline MXSN & 29 & ISLA TIBURON \\
\hline MXSN & 71 & JAPON EN MEXICO \\
\hline MXSN & 70 & LA ANTIGUA \\
\hline MXSN & 73 & LA CANDELARIA \\
\hline MXSN & 25 & LA CARIDAD \\
\hline MXSN & 61 & LA DURA \\
\hline MXSN & 67 & LA ESTRELLA \\
\hline MXSN & 62 & LA GLORIA-SANTA ROSA \\
\hline MXSN & 16 & LA GUADALUPANA ET AL \\
\hline MXSN & 72 & LA GUADALUPE \\
\hline MXSN & 10 & LA INDEPENDENCIA \\
\hline MXSN & 49 & LA PLUMOSITA \\
\hline MXSN & 37 & LA VERDE \\
\hline MXSN & 77 & LA VICTORIA (ALAMOS A) \\
\hline MXSN & 45 & LAMPAZOS \\
\hline MXSN & 14 & LAS ANTILLAS, PTO RICO, LA CUBANA \\
\hline MXSN & 15 & LAS DARDANELAS \\
\hline MXSN & 84 & LAS TABLAS \\
\hline MXSN & 26 & LOS ALISOS AREA \\
\hline MXSN & 56 & LOS BRONCES \\
\hline MXSN & 48 & LOS CRESTONES \\
\hline MXSN & 3 & LOS TANOUES \\
\hline MXSN & 83 & LUPITA \\
\hline MXSN & 80 & MARIA, LA VERDE Y CERRO BLANCO \\
\hline MXSN & 47 & MATAPE \\
\hline MXSN & 57 & MULATOS \\
\hline MXSN & 46 & NACORI-CHICO \\
\hline MXSN & 25A & NACOZARI DE GARCIA \\
\hline MXSN & 30 & NOCHE BUENA \\
\hline MXSN & 44 & ORO BLANCO \\
\hline MXSN & 64 & PIEDRA IMAN-EL VOLCAN \\
\hline MXSN & 39 & PIEDRAS NEGRAS \& LA CARIDAD \\
\hline MXSN & 51 & PUEBLA \\
\hline MXSN & 59 & SAN ANTONIO DEL COBRE \\
\hline
\end{tabular}

\begin{tabular}{|c|c|c|c|c|c|c|}
\hline \multirow{2}{*}{\multicolumn{3}{|c|}{$\begin{array}{l}\text { MN } \\
\text { TRONA }\end{array}$}} & N3O & 58 & W111 & 31 \\
\hline & \multicolumn{2}{|c|}{ TRONA } & N31 & 35 & $w 113$ & 45 \\
\hline \multirow{2}{*}{\multicolumn{3}{|c|}{$\begin{array}{l}A G \\
W\end{array}$}} & N27 & 27 & $w 109$ & 23 \\
\hline & & & N29 & 40 & $w 110$ & 16 \\
\hline \multicolumn{3}{|l|}{$\Delta \mathbf{A U}$} & N27 & 04 & $w 109$ & 10 \\
\hline \multicolumn{3}{|l|}{ SB } & N3O & 43 & $w 112$ & 10 \\
\hline \multicolumn{3}{|l|}{ ASB } & N28 & 46 & $w 109$ & 06 \\
\hline \multirow{2}{*}{\multicolumn{2}{|c|}{$\begin{array}{l}\mathrm{CU} \\
\mathrm{MN}\end{array}$}} & MO & N3O & 58 & $w 110$ & 18 \\
\hline & & & N3 1 & 15 & $w 109$ & 41 \\
\hline \multicolumn{2}{|c|}{$\triangle A G$} & & N29 & 08 & $W 109$ & 12 \\
\hline MO & \multirow{2}{*}{$P B$} & $\mathrm{cu}$ & N3O & 04 & $w 109$ & 45 \\
\hline cU & & $A G$ & N29 & $\infty 0$ & W109 & 44 \\
\hline \multicolumn{2}{|c|}{$\mathrm{N}$} & & N30 & 21 & $w 108$ & 38 \\
\hline AU & AG PB & & N3O & 10 & $W 110$ & 35 \\
\hline $\mathrm{CU}$ & MO & & N3O & 50 & $w 110$ & 09 \\
\hline SB & & & N3O & 37 & $w+12$ & 37 \\
\hline \multirow{2}{*}{\multicolumn{2}{|c|}{ U }} & & N29 & 23 & W111 & 11 \\
\hline & & & N26 & 58 & $W 108$ & 43 \\
\hline \multirow{2}{*}{\multicolumn{2}{|c|}{ G $Z N A U$}} & & N27 & 25 & $w 108$ & 45 \\
\hline & & & N27 & 12 & W108 & 50 \\
\hline \multirow{2}{*}{\multicolumn{2}{|c|}{ J $P B \quad Z N A G \quad A U$}} & & N27 & 02 & W109 & 03 \\
\hline $\mathbf{F}$ & & & N3O & 50 & $w 109$ & 22 \\
\hline $\mathrm{cu}$ & & MO & N3O & 04 & $w 112$ & 29 \\
\hline $\mathrm{CU}$ & & & N28 & 45 & $w 109$ & 40 \\
\hline MN & & & N3O & 45 & W110 & 43 \\
\hline$w$ & & & N29 & 09 & $W 110$ & 58 \\
\hline MN & & & N28 & 51 & $w 112$ & 21 \\
\hline $\mathbf{A G}$ & $Z N A U$ & & N27 & 23 & $W 108$ & 47 \\
\hline$A G$ & & & N27 & 16 & W109 & 02 \\
\hline $\mathrm{cu}$ & $A U$ & & N27 & 11 & $w 109$ & 15 \\
\hline$c U$ & & мо & N3O & 19 & $w 109$ & 33 \\
\hline MN & & & N28 & 20 & $w 109$ & 24 \\
\hline AU & & & N27 & 24 & $W 108$ & 55 \\
\hline MO & & & N28 & 20 & $w 109$ & 01 \\
\hline MN & & & N3O & 23 & $w 110$ & 50 \\
\hline CU & & $A G \quad A U$ & N27 & 18 & $W 108$ & 50 \\
\hline MN & & & N31 & 15 & $w 110$ & 52 \\
\hline $\mathrm{cu}$ & & FE & N29 & 09 & $w 109$ & 22 \\
\hline $\mathrm{CU}$ & & & N29 & 24 & $w 111$ & 20 \\
\hline$w$ & & & N27 & 03 & $W 108$ & 58 \\
\hline$A G$ & PB ZN & & N29 & 27 & $W 109$ & 30 \\
\hline MN & & & N3O & 41 & $W 110$ & 58 \\
\hline MN & & & N3O & 26 & $W 110$ & 56 \\
\hline $\mathrm{Cu}$ & & & N26 & 47 & $W 108$ & 38 \\
\hline $\mathrm{CU}$ & & MO $A G \quad P B \quad Z N$ & N3O & 24 & W 109 & 29 \\
\hline PB & $A G A U C U$ & & N28 & 37 & $w 109$ & 18 \\
\hline AG & PB ZN & & N29 & 11 & W109 & 39 \\
\hline AU & & & N3 1 & 40 & $w 112$ & 57 \\
\hline $\mathrm{cu}$ & & & N26 & 54 & $W 108$ & 45 \\
\hline $\mathrm{cu}$ & $A U$ & FE & N26 & 58 & $W 108$ & 35 \\
\hline TLC & & & N29 & 10 & $W 109$ & 56 \\
\hline $\mathrm{CU}$ & $A G$ & & N28 & 35 & $w 109$ & 08 \\
\hline $\mathrm{cu}$ & & & N29 & 28 & $W 108$ & 43 \\
\hline $\mathrm{cU}$ & & & N3O & 26 & $W 109$ & 42 \\
\hline PB & $Z N A G$ & $A U C U$ & N27 & 55 & $w+11$ & 08 \\
\hline NA & & & N29 & 47 & W109 & 08 \\
\hline $\mathrm{FE}$ & & & N27 & 45 & $W 109$ & 27 \\
\hline $\mathrm{FE}$ & & & N29 & 14 & $W 111$ & 04 \\
\hline $\mathrm{CU}$ & & & N28 & 59 & $W 109$ & 30 \\
\hline $\mathrm{CU}$ & & $u$ & N28 & 37 & $W 109$ & 37 \\
\hline
\end{tabular}




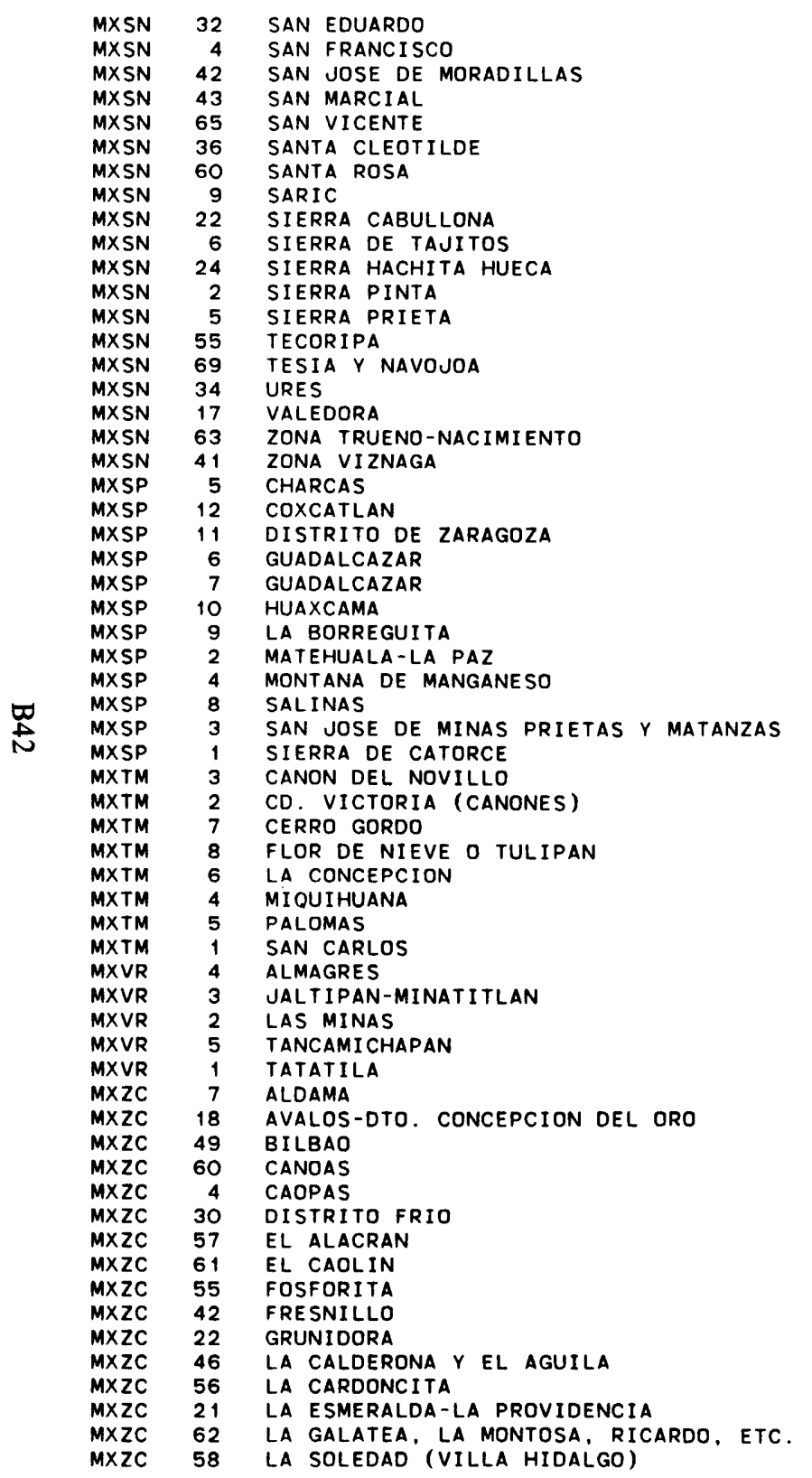

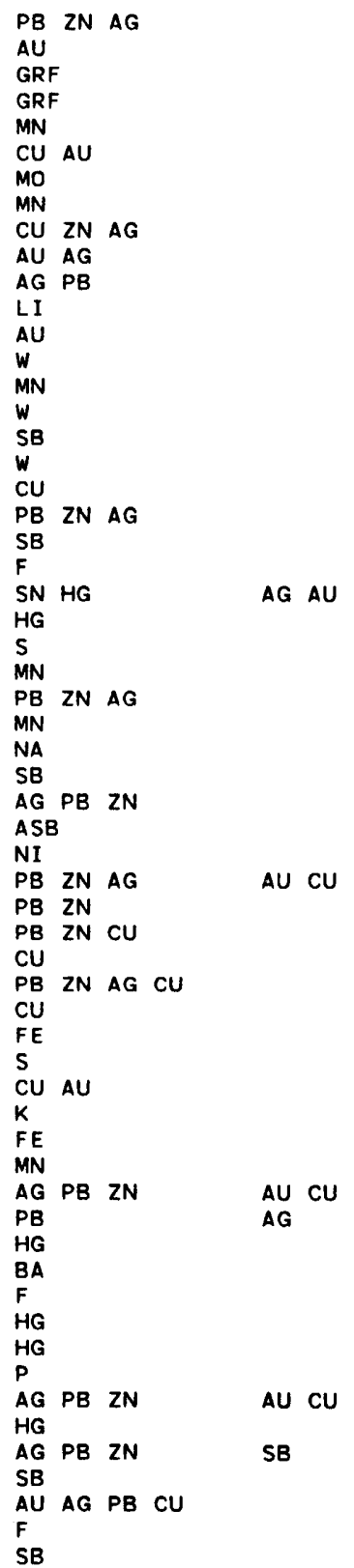

\begin{tabular}{|c|c|c|c|c|}
\hline N29 & 30 & $w 111$ & 45 & 707C7A7 \\
\hline N3 1 & 30 & W113 & 02 & 4A7C7A7 \\
\hline N28 & 35 & W110 & 29 & TIE 15A 1OA4 \\
\hline N28 & 30 & W110 & 20 & IIE 15B IOA4 \\
\hline N27 & 30 & W109 & 44 & 10A7C7A7 \\
\hline N29 & 20 & W11 & 50 & 3E 14C3A7 - \\
\hline N28 & 25 & W109 & 10 & 3B4C7A7 \\
\hline N31 & 10 & Wi11 & 22 & 10A7C7A7 \\
\hline N31 & 05 & $W 109$ & 31 & 3D 14C7A6 \\
\hline N3O & 58 & $w 112$ & 15 & 4C 1 C7A 7 \\
\hline N3O & 35 & W108 & 52 & 4D7C7A7 \\
\hline N31 & 41 & $W 113$ & 10 & IE 15C 1A4 \\
\hline N31 & 18 & $w+13$ & 02 & 4A7C7A7 \\
\hline N28 & 38 & $W 109$ & 57 & 1A4C7A7 \\
\hline N27 & 20 & $W 109$ & 20 & 10A7C7A? \\
\hline N29 & 27 & $w 110$ & 29 & 1A 14C3A7 \\
\hline N3O & 22 & $w+11$ & 30 & $8 B 1 C 7 A 7$ \\
\hline N28 & 15 & W109 & 44 & 1АЗ $7 C 4 A 8$ \\
\hline N28 & 55 & W111 & 06 & 3A4C7A \\
\hline N23 & 11 & W101 & 04 & $70147 A 7 A 7$ \\
\hline N22 & $\infty$ & W098 & 58 & $8 B 14 C 7 A$ \\
\hline N21 & 58 & $W 100$ & 35 & 8C $17 A 7 A 7$ \\
\hline N22 & 44 & W100 & 25 & $1814 C 4 A B$ \\
\hline N22 & 42 & W100 & 11 & 8A1C7A \\
\hline N22 & 14 & W100 & 18 & 11A 1B5A7 \\
\hline N22 & 16 & W100 & 05 & 1OA37B5A4 \\
\hline N23 & 44 & $W 100$ & 45 & 7047A7A7 \\
\hline N23 & 21 & W101 & 42 & 1OA 14B5A7 \\
\hline N22 & 36 & W101 & 46 & GB ICGA \\
\hline N23 & 36 & W100 & 59 & $881 B \quad 10 B 7$ \\
\hline N23 & 44 & W100 & 55 & $4014 A 7 A 7$ \\
\hline N23 & 41 & wo9g & 16 & SE $158 \mathrm{C}$ 1OA2 \\
\hline N23 & 43 & wo9g & 16 & $5 B 15 C 5 A 2$ \\
\hline N23 & 14 & wo99 & $\infty 0$ & 7D1B7A7 \\
\hline N23 & 09 & wo99 & 05 & 7C 1C $10 A 7$ \\
\hline N23 & 17 & WO99 & 06 & 7C 1C 1OA7 \\
\hline N23 & 36 & w099 & 50 & 3А 1C7A7 \\
\hline N23 & 25 & W099 & 55 & $701 C 7 A 7$ \\
\hline N24 & 32 & W098 & 58 & 3А2C7A7 \\
\hline N17 & 48 & W094 & 55 & 2A1B2A4 \\
\hline N17 & 57 & W097 & 45 & 11А37A5A7 \\
\hline N19 & 40 & W097 & 14 & उE $14 C 3 A 7$ \\
\hline N17 & 45 & W094 & 45 & 6C37A6A4 \\
\hline$N 19$ & 42 & W097 & 08 & $2 A 14 C 3 A 7$ \\
\hline N24 & 17 & W103 & 18 & 10A7C7A7 \\
\hline N24 & 37 & $w+01$ & 30 & 4014A5A7 \\
\hline N22 & 38 & $W+02$ & 10 & 7A 14C7A7 \\
\hline N22 & 12 & Wto1 & 55 & 8A7B7A7 \\
\hline N24 & 37 & W102 & $\infty$ & BD 1C7A7 \\
\hline N23 & 39 & W103 & 32 & 8C1C7A7 \\
\hline N22 & 28 & W101 & 56 & BA 1C5A7 \\
\hline N22 & 06 & W101 & 50 & BA7C5A7 \\
\hline N23 & 54 & W101 & 59 & 2E7C5A7 \\
\hline N23 & 15 & $w 102$ & 50 & 4D $1 A 7 A 7$ \\
\hline N24 & 15 & W101 & 58 & 8A1C5A7 \\
\hline N22 & 55 & W102 & 16 & 4D1C7A7 \\
\hline N23 & 42 & W101 & 51 & 8B5C5A7 \\
\hline N24 & 23 & W100 & 58 & 4C4C7A7 \\
\hline N21 & 40 & W103 & 05 & 8C7C7A7 \\
\hline N22 & 24 & W101 & 40 & 8B7B7A7 \\
\hline
\end{tabular}




\begin{tabular}{|c|c|c|}
\hline$M \times Z C$ & 39 & LA TINAJA \\
\hline$M \times Z C$ & 45 & LA TRINCHERA \\
\hline$M \times Z C$ & 54 & LA VUELTA DE GABRIEL \\
\hline$M \times Z C$ & 38 & LOS LAMENTOS \\
\hline$M \times Z C$ & 63 & LOS LUGOS Y SAN MIGUEL \\
\hline$M \times Z C$ & 23 & MAHOMA \\
\hline$M \times Z C$ & 41 & MARAVILLAS \\
\hline$M \times Z C$ & 52 & MINAS DE MILAGROS \\
\hline$M \times Z C$ & 40 & MOLLEDA \\
\hline$M \times Z C$ & 13 & NIEVES \\
\hline$M \times Z C$ & 17 & NOCHE BUENA \\
\hline$M \times Z C$ & 10 & NUEVO MERCURIO \\
\hline$M \times Z C$ & 32 & P.V. 13 \\
\hline$M \times Z C$ & 26 & P.V. 2 \\
\hline$M \times Z C$ & 43 & P.V. 23 \\
\hline$M \times Z C$ & 1 & P.V. 26 \\
\hline$M \times Z C$ & 48 & P.V. 26 (2) \\
\hline$M X Z C$ & 16 & P.V. 27 \\
\hline$M \times Z C$ & 29 & P.V. 27 (SAN MARTIN) \\
\hline$M \times Z C$ & 9 & P.V. 3, EL REY BALTAZAR, LA PRINGA \\
\hline$M \times Z C$ & 11 & P.V. 30 \\
\hline$M \times Z C$ & 2 & P.V. 45 (EL CARRIZO) \\
\hline$M \times Z C$ & 12 & P.V. 47 \\
\hline MXZC & 19 & P.V. 49 \\
\hline$M \times Z C$ & 34 & P.V. 49 (TENANGO) \\
\hline$M \times Z C$ & 53 & P.V. 6 (LA BLANQUITA) \\
\hline$M \times Z C$ & 27 & P.V. 7 \\
\hline$M \times Z C$ & 33 & P.V. 9 (MARGARITA) \\
\hline$M \times Z C$ & 50 & P.V. 9 (SANTA CRUZ) \\
\hline$M \times Z C$ & 14 & PACHECO \\
\hline$M \times Z C$ & 51 & PENASCO AMARILLO \\
\hline$M \times Z C$ & 25 & PLAN DE ESTANERAS \\
\hline$M \times Z C$ & 31 & SAIN ALTO \\
\hline$M \times Z C$ & 15 & SAN BENITO \\
\hline$M \times Z C$ & 24 & SAN FCO. LA VALENCIANA, LA LEGION \\
\hline$M \times Z C$ & 8 & SAN FRANCISCO \\
\hline$M \times Z C$ & 6 & SAN MIGUEL AUZA \\
\hline$M \times Z C$ & 44 & SANTA ELENA \\
\hline$M \times Z C$ & 59 & SIERRA DE PINOS \\
\hline MXZC & 3 & SIN NOMBRE \\
\hline$M \times Z C$ & 28 & SOMBRERETE \\
\hline$M \times Z C$ & 20 & TANQUECITO \\
\hline$M \times Z C$ & 35 & TENANGO, LA PIROLUSITA, ETC. \\
\hline$M \times Z C$ & 5 & TEYRA \\
\hline$M \times Z C$ & 37 & 2. LA MANGANITA \\
\hline$M \times Z C$ & 36 & Z. SAN FELIPE DE JESUS \\
\hline$M \times Z C$ & 47 & ZACATECAS \\
\hline NUNU & 21 & AMERICA \\
\hline NUNU & 18 & BONANZA \\
\hline NUNU & 8 & DIPILTO \\
\hline NUNU & 13 & EL CHORREADERO \\
\hline NUNU & 14 & EL LIMON \\
\hline NUNU & 4 & LA CORONA \\
\hline NUNU & 12 & LA GRECIA \\
\hline NUNU & 15 & LA INDIA \\
\hline NUNU & 24 & LA LIBERTAD \\
\hline NUNU & 17 & LA LUZ \\
\hline NUNU & 22 & LA REINA \\
\hline NUNU & 9 & MACUELIZO 1 \\
\hline NUNU & 10 & MACUELIZO 2 \\
\hline
\end{tabular}

\begin{tabular}{|c|c|c|c|c|c|c|c|}
\hline \multicolumn{3}{|l|}{ MN } & N23 & 29 & W 102 & 39 & $10 A 1 C 11 A 7$ \\
\hline \multicolumn{8}{|r|}{ 8C7C7A7 } \\
\hline $\mathrm{CU}$ & & & N22 & 13 & W103 & 31 & 3А $7 C 5 A 7$ \\
\hline MN & & & N23 & 35 & W 102 & 33 & $10 A 1 C 11 A 7$ \\
\hline$A U$ & & & N21 & 10 & W103 & 25 & 4A4C7A7 \\
\hline HG & & & N24 & 10 & W 101 & 40 & 8A 1C5A7 \\
\hline HG & & & N23 & 19 & W103 & 11 & 8А7C7A7 \\
\hline AG & & PB ZN AU & N22 & 30 & $W 102$ & 12 & 4D7C7A7 \\
\hline MN & & & N23 & 25 & W 102 & 35 & 10A7C7A7 \\
\hline MN & & & N24 & 02 & $W 102$ & 52 & 10A7C7A7 \\
\hline PB & $Z N A G$ & $A U C U$ & N24 & 43 & W101 & 37 & $7014 B 5 A 7$ \\
\hline HG & & & N24 & 14 & W102 & 10 & 8A $1 A 7 A 7$ \\
\hline $\mathbf{P}$ & & & N23 & 41 & $W 102$ & 50 & $2 E 4 C 5 A 7$ \\
\hline AG & PB & & N23 & 58 & $w 103$ & 03 & 4D $1 C 7 A 7$ \\
\hline HG & & & N23 & 16 & W102 & 17 & 8A $1 C 10 A 7$ \\
\hline $\mathrm{CU}$ & & & N24 & 52 & $W 102$ & 10 & $3 A 1 C 5 A 7$ \\
\hline $\mathbf{P}$ & & & N22 & 46 & $W 102$ & 09 & 2E 7C $10 A 7$ \\
\hline AG & P.B & & N24 & 53 & W101 & 50 & 4D 1C7A7 \\
\hline AG & PB CU & & N23 & 40 & W103 & 45 & 4D 1B7A7 \\
\hline$H G$ & & & N24 & 15 & $W 102$ & 44 & 8A 1C5A7 \\
\hline HG & & & N24 & 09 & $W 102$ & 55 & 8А2C7A7 \\
\hline BA & & & N24 & 48 & $W 102$ & 04 & 8D 1C7A7 \\
\hline SB & & & N24 & 08 & $W 102$ & 44 & $8 B 1 C 7 A 7$ \\
\hline PB & $Z N$ & & N24 & 31 & W101 & 47 & 7C $1 C 7 A 7$ \\
\hline MN & & & N23 & 32 & $W 102$ & 18 & 1OA7C 11A7 \\
\hline AG & PB & & N22 & 27 & $W 102$ & 07 & 4D4C7A7 \\
\hline HG & & SB & N23 & 56 & $W 102$ & 27 & 8A $1 C 7 A 7$ \\
\hline MN & & $\mathbf{P}$ & N23 & 42 & W102 & 20 & 10A 1C7A7 \\
\hline SN & & & N22 & 28 & $W 102$ & 34 & $187 C 7 A 7$ \\
\hline SB & & & N24 & 02 & $W 102$ & 30 & $8 B+C 5 A 7$ \\
\hline MN & & & N22 & 27 & W 102 & 25 & 10A7C7A7 \\
\hline SN & & & N22 & 45 & W 104 & 01 & 1B7B7A7 \\
\hline HG & & & N23 & 35 & W103 & 13 & 8А7A7A7 \\
\hline AU & & & N25 & 01 & $w 101$ & 59 & AA $1 C 7 A 7$ \\
\hline CU & $A G \quad A U$ & & N24 & 14 & W101 & 10 & SE $1 C 7 A 7$ \\
\hline SB & & & N24 & 18 & W103 & oo & $8 B\{C 5 A 7$ \\
\hline SN & & & N24 & 20 & W103 & 28 & 1B7C7A7 \\
\hline AG & & & N22 & 48 & W103 & 33 & 4D4C7A7 \\
\hline SN & & & N22 & 18 & W101 & 32 & 1B7C7A7 \\
\hline TLC & & & N24 & 45 & W102 & 10 & 10E 15C5A6 \\
\hline AG & PB ZN & $A U C U$ & N23 & 40 & W103 & 40 & 4D1B7A7 \\
\hline HG & & & N24 & 25 & W101 & 40 & $8 A 1 C 5 A 7$ \\
\hline MN & & & N23 & 31 & $W 102$ & 11 & 1OA 1C $11 A 7$ \\
\hline$A U$ & & & N24 & 34 & W 102 & 10 & 4A4C7AG \\
\hline MN & & & N23 & 25 & $W 102$ & 20 & 1OA 1C 1 १A \\
\hline MN & & & N23 & 28 & W.102 & 12 & 1OA 1C $11 A 7$ \\
\hline $\mathbf{A G}$ & PB ZN & $A U C U$ & N22 & 45 & W102 & 35 & 4D 15A7A7 \\
\hline AU & & & $\mathrm{N} 13$ & 47 & W084 & 30 & 4A7C7A7 \\
\hline$A U$ & AG PB ZN & & $N 13$ & 58 & W084 & 37 & 407B7A7 \\
\hline$A U$ & & & N13 & 45 & W086 & 32 & 4A 15C7A5+ \\
\hline AU & & & $N+3$ & $\infty$ & W086 & 49 & 4А7C7A7 \\
\hline AU & & & N12 & 44 & W086 & 43 & 4A7B7A7 \\
\hline$A U$ & & & $\mathrm{~N} 13$ & 48 & W086 & 01 & 4A 15C7A5+ \\
\hline AU & AG & & $N+3$ & 10 & W086 & 35 & $4 C 7 C 5 A 7$ \\
\hline$A U$ & & $\mathbf{A G}$ & $\mathrm{N} 12$ & 45 & W086 & 11 & 4A7C7A7 \\
\hline$A U$ & AG & & N12 & 17 & W085 & 09 & 4C7C7A7 \\
\hline$A U$ & $\mathrm{CU}$ & $\mathbf{A G}$ & $\mathrm{N} 13$ & 45 & W084 & 48 & 4B14B5A6+ \\
\hline$A U$ & AG & & $\mathrm{N} 12$ & 58 & W085 & 49 & 4C7C7A7 \\
\hline$w$ & MO & & $\mathrm{N} 13$ & 42 & W086 & 37 & 1A145C3A5+ \\
\hline AG & PB ZN & & $\mathrm{N} 13$ & 37 & W086 & 37 & 40145C7A5+ \\
\hline
\end{tabular}




\begin{tabular}{|c|c|c|c|}
\hline $\begin{array}{l}\text { NUNU } \\
\text { NUNU }\end{array}$ & $\begin{array}{r}19 \\
5\end{array}$ & $\begin{array}{l}\text { MONTE CARMELO } \\
\text { MURRA }\end{array}$ & \\
\hline NUNU & 16 & OCONGUAS & \\
\hline NUNU & 11 & PALACAGUINA & \\
\hline NUNU & 2 & PIS PIS & \\
\hline NUNU & 1 & RIO WAWA & \\
\hline NUNU & 3 & RISCOS DE ORO & \\
\hline NUNU & 20 & ROSITA & \\
\hline NUNU & 6 & SAN ALBINO & \\
\hline NUNU & 7 & SAN JUAN TELPANECA & \\
\hline NUNU & 25 & SANTO DOMINGO & \\
\hline NUNU & 23 & VERDE & \\
\hline PNPN & 4 & BOCAS DEL TORO & \\
\hline PNPN & 3 & BOQUETE & \\
\hline PNPN & 5 & CALDERA 1 & \\
\hline PNPN & 6 & CALDERA 2 & \\
\hline PNPN & 37 & CANAS & \\
\hline PNPN & 2 & CANAS GORDAS & \\
\hline PNPN & 16 & CANAZAS & \\
\hline PNPN & 1 & CEBU & \\
\hline PNPN & 14 & CERRO BANCO & \\
\hline PNPN & 29 & CERRO CABRA & \\
\hline PNPN & 30 & CERRO CAMPANA & \\
\hline PNPN & 15 & CERRO COLORADO & \\
\hline PNPN & 9 & CERRO PETAQUILLA & \\
\hline PNPN & 31 & CHAME & \\
\hline PNPN & 11 & COCUYO & \\
\hline PNPN & 8 & DAVID & \\
\hline PNPN & 19 & LAS LAJAS & \\
\hline PNPN & 21 & LAS PALMAS & \\
\hline PNPN & 18 & LOS HATILLOS & \\
\hline PNPN & 23 & LOS POZOS & \\
\hline PNPN & 26 & MANDINGA AREA & \\
\hline PNPN & 25 & NOMBRE DE DIOS-RIO BOQUERON (HYATT) & AREA \\
\hline PNPN & 12 & NUESTRO AMO & \\
\hline PNPN & 24 & PALO SECO & \\
\hline PNPN & 7 & PUERTO ARMUELLES & \\
\hline PNPN & 17 & REMANSE & \\
\hline PNPN & 28 & RIO BAYANO & \\
\hline PNPN & 10 & RIO INDIO & \\
\hline PNPN & 22 & RIO LOVAINA & \\
\hline PNPN & 36 & RIO PINA & \\
\hline PNPN & 32 & RIO SAMBU & \\
\hline PNPN & 35 & RIO TUCUTI & \\
\hline PNPN & 13 & SERRANIA DE TABASARA & \\
\hline PNPN & 20 & TOLE & \\
\hline PNPN & 34 & TUQUESA & \\
\hline RORO & 1 & AGUADA & \\
\hline RORO & 10 & CARMEN & \\
\hline RQRO & 4 & COROZAL (PALOS BLANCOS) & \\
\hline RORQ & 9 & JUANA DIAZ MINE & \\
\hline RQRQ & 11 & KEYSTONE (JUNCOS) MINE & \\
\hline RQRO & 12 & LA MINA (RIO BLANCO) PROSPECT & \\
\hline RQRO & 5 & LA MUOA & \\
\hline RORQ & 6 & MAYAGUEZ (GUANAJIBO, LAS MESAS) & \\
\hline RORO & 8 & MINILLAS, SAN GERMAN & \\
\hline RORQ & 3 & RIO VIVI (CALA ABAJO, PIEDRA HUECA) & \\
\hline RORO & 7 & ROSARIO-MARICAO AREA & \\
\hline RORQ & 2 & TANAMA (HELECHO, LOS ANGELES) & \\
\hline UKUK & 1 & BELMONT BARYTES QUARRY & \\
\hline
\end{tabular}

\begin{tabular}{|c|c|c|c|c|c|c|c|}
\hline \multicolumn{3}{|l|}{ FE } & $N 13$ & 58 & W084 & 26 & $2 A 47 C 10 A 7$ \\
\hline \multirow{2}{*}{\multicolumn{3}{|c|}{$A U \bar{~}$}} & N13 & 43 & W086 & 03 & $4 A 15 C 7 A 5+$ \\
\hline \multirow{2}{*}{\multicolumn{3}{|c|}{$\begin{array}{l}A U \\
\text { SB }\end{array}$}} & N13 & 45 & W085 & 47 & $\triangle A 3 C 4 A B$ \\
\hline & & & $\mathrm{N} 13$ & 29 & W086 & 25 & $8 B 15 C 7 A 7$ \\
\hline \multicolumn{3}{|l|}{$\begin{array}{l}\text { SB } \\
A U\end{array}$} & $\mathrm{~N} 14$ & 06 & WO84 & 37 & $4 A 3 C 4 A 8$ \\
\hline \multicolumn{3}{|l|}{$\Delta U$} & N14 & 18 & WO8 4 & 23 & $4 A 3 C 4 A 8$ \\
\hline \multicolumn{3}{|c|}{$\mathrm{AU} C U$} & N14 & 03 & W08 4 & 12 & AB 7C $7 A 7$ \\
\hline \multirow{2}{*}{\multicolumn{3}{|c|}{$\begin{array}{l}U \\
U\end{array}$}} & $\mathrm{~N} 13$ & 55 & WO84 & 22 & 3E $14 B 3 A 6$ \\
\hline & & & N13 & 41 & W086 & 07 & $4 A 15 C 7 A 5+$ \\
\hline \multicolumn{3}{|l|}{$\mathrm{AU}$} & $\mathrm{N} 13$ & 34 & W086 & 13 & $4 A 15 C 7 A 5+$ \\
\hline \multicolumn{3}{|l|}{$A U$} & $\mathrm{~N} 12$ & 13 & W085 & 01 & 4A7C7A7 \\
\hline \multicolumn{3}{|l|}{ AU } & $\mathrm{N} 12$ & 54 & W085 & 49 & 4A7C7A7 \\
\hline \multirow{2}{*}{\multicolumn{3}{|c|}{ AU }} & N8 & 55 & WO82 & 12 & 4A7C7A 7 \\
\hline & & & N8 & 52 & W082 & 27 & 4A 7C7A7 \\
\hline \multicolumn{3}{|l|}{$\begin{array}{l}\mathrm{AU} \\
\mathrm{CU}\end{array}$} & NB & 44 & WO82 & 20 & 3A7C7A7 \\
\hline \multicolumn{3}{|l|}{$A U$} & N8 & 40 & W082 & 21 & 4A $7 C 7 A 7$ \\
\hline \multicolumn{3}{|c|}{$u_{u} c u$} & N7 & 47 & W077 & 35 & 4B7C7A7 \\
\hline$A U$ & & & N8 & 50 & WO82 & 49 & 4A7C7A7 \\
\hline$\Delta U$ & & & N8 & 22 & W081 & 10 & 4A 7C7A7 \\
\hline $\mathrm{FE}$ & & & N9 & 30 & W082 & 25 & $2 A 37 C 4 A 8$ \\
\hline$C U$ & & & N8 & 27 & W081 & 36 & ЗАЗС $7 A$ \\
\hline$A U$ & & & N9 & 00 & W079 & 40 & 4А 7C7A7 \\
\hline $\mathrm{AU}$ & $A G$ & & N8 & 45 & W079 & 56 & 4C7C7A7 \\
\hline CU & MO & & N8 & 20 & W081 & 26 & $3 C 45 A 5 A 7$ \\
\hline cu 1 & мо & & N8 & 50 & W080 & 40 & 3C47A5A7 \\
\hline $\mathrm{FE}$ & & & N8 & 38 & W079 & 48 & $2 A 37 C 4 A 8$ \\
\hline$A U$ & & & N8 & 48 & W081 1 & $\infty 0$ & $\triangle A 7 C 7 A 7$ \\
\hline$A L$ & & & N8 & 23 & W082 & 20 & 1OB37B9A8 \\
\hline$A L$ & & & N8 & 14 & W081 & 52 & 1OB $37 C 9 A 8$ \\
\hline$A L$ & & & N8 & 05 & W08 1 & 28 & 1OB $37 C 9 A 8$ \\
\hline AU & PB & & N8 & 16 & W080 & 57 & 4D7C7A7 \\
\hline $\mathrm{CU}$ & & & N7 & 52 & WO80 & 39 & उA7C $10 A 7$ \\
\hline MN & & & N9 & 30 & W079 & 04 & 1OA5C $11 A 5$ \\
\hline MN & & & N9 & 30 & W079 & 30 & 10A5C $11 A 5$ \\
\hline$A U$ & & & N8 & 29 & Wo8o & 30 & 4A7C7A7 \\
\hline$A U$ & & & N7 & 35 & W080 & 58 & $\triangle A 37 C 4 A 8$ \\
\hline $\mathrm{FE}$ & & & N8 & 17 & W082 & 42 & $2 A 37 C 4 A 8$ \\
\hline$A U$ & & & N8 & 17 & W081 & 04 & 4A7C $7 A 7$ \\
\hline$A U$ & & & N9 & 05 & W078 & 20 & $\triangle A 3 C 4 A 8$ \\
\hline $\mathrm{AU}$ & & & NB & 49 & W080 & 03 & 4A 7C7A 7 \\
\hline$A U$ & & & N7 & 52 & W081 & 33 & 4A7C7A7 \\
\hline$A U$ & & & N7 & 40 & WO78 & 08 & 4A56C7A7 \\
\hline AU & & & N8 & 04 & W078 & 12 & $\triangle A 3 C 4 A 8$ \\
\hline$\Delta U$ & & & N8 & 06 & W077 & 55 & $\triangle A 3 C 4 A B$ \\
\hline $\mathrm{cu}$ & & & NB & 34 & WO81 & 50 & ЗА $3 C 10 A 7$ \\
\hline$A L$ & & & N8 & 14 & WO8 1 & 41 & 1ОВ37B9A8 \\
\hline$A U$ & $\mathrm{CU}$ & & N8 & 30 & W077 & 35 & AB 7B $7 A 7$ \\
\hline MN & & & N18 & 22 & W067 & 10 & 1OA5C7A6 \\
\hline$A U$ & $A G$ & PB ZN CU & N18 & 02 & W066 & 10 & 4D5C7A5+ \\
\hline$\widehat{A U}$ & & & N18 & 17 & W066 & 17 & $\triangle A 3 C 4 A 8$ \\
\hline MN & & & N18 & 04 & W066 & 28 & 1OA5C11A5 \\
\hline$F E$ & & cu & N18 & 14 & W065 & 54 & $2045 C 345+$ \\
\hline CU & & & $N 18$ & 16 & W065 & 49 & $3 A 45 C 3 A 5+$ \\
\hline CU 1 & мо & & N18 & 19 & W066 & 05 & $3 C 45 C 5 A 6$ \\
\hline FE & NI CO & & N18 & 09 & W067 & 08 & $5 B 58 B 9 A 7+$ \\
\hline AU & $\mathrm{CU}$ & $A G P B$ & N18 & 04 & W067 & 00 & 4B5C7A6 \\
\hline $\mathrm{CU}$ & & AU & N18 & 12 & W066 & 41 & उA45B5A6 \\
\hline FE & NI $\mathrm{CO}$ & & N18 & 08 & W067 & $\infty$ & $5 B 58 C 9 A 7+$ \\
\hline $\mathrm{cU}$ & & $A U$ & N18 & 15 & W066 & 47 & 3A45B5A6 \\
\hline BA & & & N17 & 05 & W06 1 & 50 & 8D7C7A7 \\
\hline
\end{tabular}




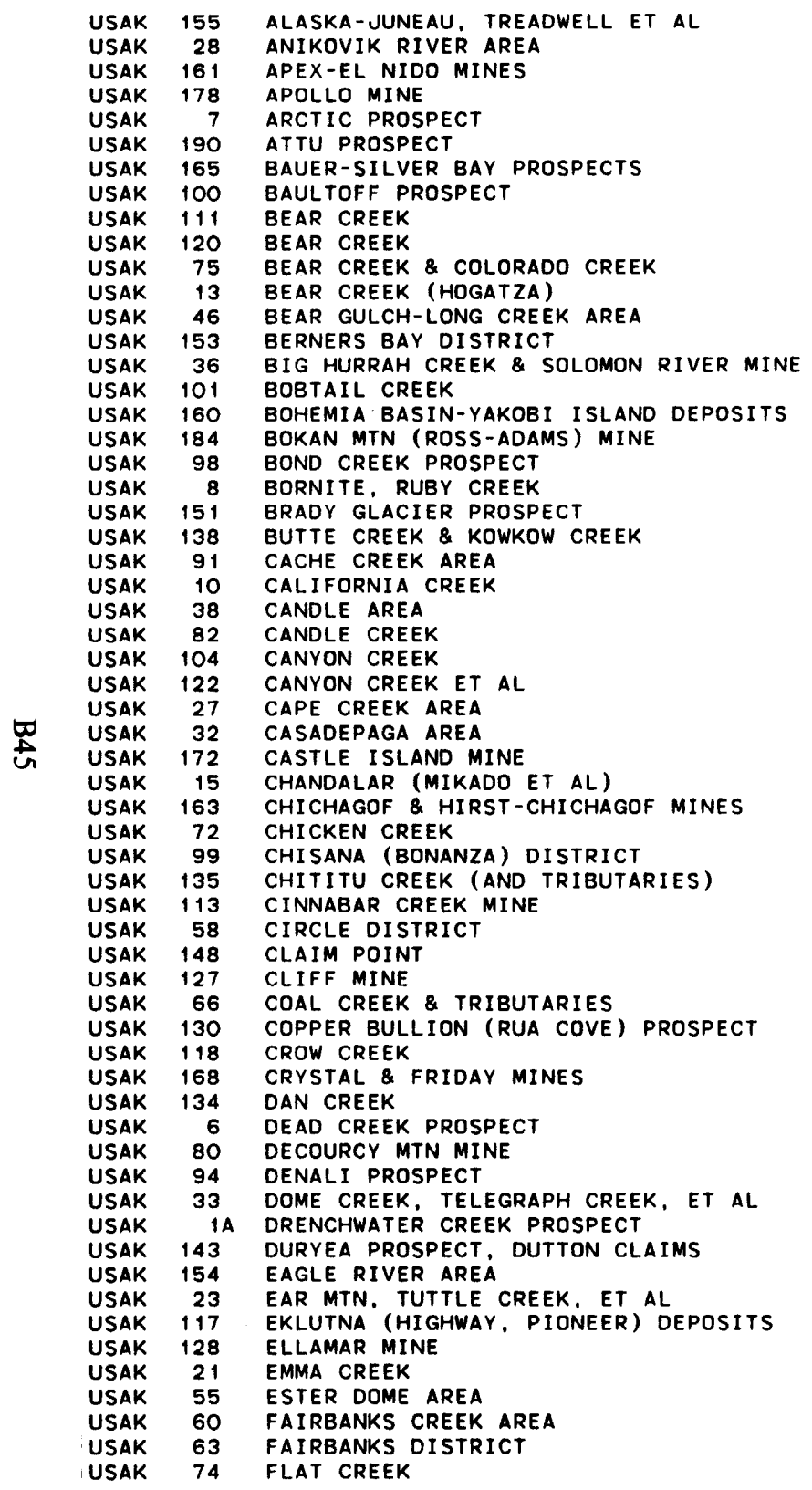

\begin{tabular}{|c|c|c|c|c|c|c|c|c|c|c|}
\hline AU & & $A G$ & PB & & & N58 & 17 & $W 134$ & 21 & $4 A 145 B 7 A$ \\
\hline AU & SN & $\mathbf{w}$ & & & & N65 & 32 & $W 167$ & 40 & 4A3C4AB \\
\hline AU & & AG & w & & & N57 & 57 & $w 136$ & 17 & 4A456C7A4+ \\
\hline AU & & AG & PB & $2 N$ & $\mathrm{CU}$ & N55 & 11 & $W 160$ & 35 & $4 A 45 C 7 A 7$ \\
\hline \multirow[t]{2}{*}{$\mathrm{CU}$} & $\mathrm{ZN}$ & PB & $\Delta G$ & $\mathbf{A U}$ & & N67 & 11 & $W 156$ & 22 & $3 D 5 A 11 A 3$ \\
\hline & & $\mathrm{CU}$ & & & & N52 & 52 & E 172 & 50 & $3 A 45 C 5 A 7$ \\
\hline \multirow[t]{2}{*}{$A U$} & & & & & & N57 & $\infty$ & W135 & 09 & $4 A 5 C$ \\
\hline & & $\mathrm{CU}$ & & & & N62 & 06 & $W 141$ & 14 & 3A456B5A5 \\
\hline$A U$ & & HG & & & & N6 1 & 03 & $W 159$ & 49 & $4 A 3 C 4 A 7+$ \\
\hline$A U$ & & $\mathbf{A G}$ & & & & N6O & 55 & $W 149$ & 34 & $4 A 3 C 4 A 8$ \\
\hline$A U$ & & & & & & N63 & 35 & $W 156$ & 04 & 4A3C4A8 \\
\hline AU & & SN & PT & & & N66 & 12 & W155 & 42 & $\triangle A 3 C 4 A B$ \\
\hline$A U$ & & SN & & & & N64 4 & 24 & W155 & 32 & 4A3C4A8 \\
\hline AU & & & & & & N58 & 52 & $w 135$ & 05 & 4A456C5A \\
\hline AU & & $w$ & & & & N64 & 39 & $W 164$ & 19 & 4AC7A \\
\hline$A U$ & & HG & & & & N6 1 & 55 & $W 161$ & 25 & СA3C4A8 \\
\hline NI & $\mathrm{CU}$ & & & & & N57 & 59 & W136 & 26 & $5056 B 8 A$ \\
\hline$u$ & $\mathrm{TH}$ & & & & & N54 & 55 & $W 132$ & 08 & 9E 125B5A2+ \\
\hline CU & MO & $A U$ & $A G$ & & & N62 & 12 & $W 142$ & 43 & 3C45B5A5 \\
\hline $\mathrm{CU}$ & $\mathbf{Z N}$ & PB & AG & $A U$ & $u$ & N67 & 04 & $w 156$ & 56 & 3D 1B 10A3+ \\
\hline NI & cu & Co & & & & N58 & 33 & $W 136$ & 56 & $508 C 8 A$ \\
\hline$A U$ & & PT & & & & N59 & 29 & $W 161$ & 27 & $4 A 3 C 4 A 7$ \\
\hline AU & & $\mathbf{A G}$ & PT & TH & & N62 & 31 & W150 & 55 & $4 A 3 C 4 A$ \\
\hline AU & & & & & & N66 & 58 & W156 & 37 & 4A3C4A8 \\
\hline AU & & & & & & N65 & 54 & $W 161$ & 59 & $\triangle A C 4 A 8$ \\
\hline AU & HG & $\mathbf{w}$ & & & & N62 & 53 & $W 155$ & 47 & पA3C $4 A B$ \\
\hline AU & & & & & & N60 & 11 & $W 160$ & $\infty$ & 4A3C4A6+ \\
\hline$A U$ & & $A G$ & & & & N60 & 46 & $W 149$ & 26 & $4 A 3 C 4 A 8$ \\
\hline SN & & $\mathbf{w}$ & & & & N65 & 36 & $W 167$ & 59 & 1B 14C \\
\hline AU & & & & & & N64 & 54 & $W 164$ & 15 & $\triangle A 3 C 4 A B$ \\
\hline BA & & & & & & N56 & 39 & W133 & 14 & $805 B 11 A 4$ \\
\hline AU & & & & & & N67 & 32 & $W 148$ & 14 & $4 A 15 C 7 A$ \\
\hline AU & & $A G$ & & & & N57 & 40 & $W 136$ & 06 & 4A45B7A4+ \\
\hline$A U$ & & $\mathbf{w}$ & & & & N64 & 06 & $W 141$ & 56 & $4 A 3 C 4 A 8$ \\
\hline AU & & & & & & N62 & 06 & $W 141$ & 53 & $4 A 3 C 4 A 8$ \\
\hline AU & & AG & $\mathrm{CU}$ & & & N6 1 & 16 & $W 142$ & 36 & $4 A 3 C 4 A 8$ \\
\hline HG & & SB & & & & N60 & 48 & $W 158$ & 50 & $8 A 56 C 7 A$ \\
\hline$A U$ & & SN & $w$ & & & N65 & 27 & $W 145$ & $03 *$ & 4A3C4B8 \\
\hline$C R$ & & & & & & N59 & 13 & $W 151$ & 49 & 5A58B5A4+ \\
\hline AU & & $\mathrm{CU}$ & & & & N6 1 & 09 & $W 146$ & 37 & $4 A 5 C 7 A$ \\
\hline$A U$ & & TH & & & & N65 & 18 & $W 143$ & 11 & $4 A 3 C 4 A B$ \\
\hline $\mathrm{CU}$ & & $\mathrm{ZN}$ & & & & N60 & 21 & $W 147$ & 38 & $3 A 5 C 11 A 6$ \\
\hline AU & & $A G$ & $\mathrm{Cu}$ & & & N60 & 59 & $W 149$ & 05 & 4A3C4A \\
\hline$A U$ & & & & & & N57 & 58 & W133 & 48 & 4A456C7A \\
\hline$A U$ & & $A G$ & $\mathrm{CU}$ & & & N6 1 & 22 & $W 142$ & 34 & $4 A 3 C 4 A 8$ \\
\hline $\mathrm{CU}$ & $\mathbf{Z N}$ & $A G$ & PB & & & N67 & 14 & $W 156$ & 31 & $3 D 5 C 11 A 3$ \\
\hline HG & & SB & & & & N62 & 04 & $W 158$ & 27 & $8 A 5 C 7 A 7$ \\
\hline $\mathrm{cu}$ & & & & & & N63 & 09 & $W 147$ & 08 & $3 A 5 C 11 A 4$ \\
\hline AU & & & & & & N64 & 52 & $W 164$ & 38 & $\triangle A 3 C 4 A B$ \\
\hline PB & & cu & & & & N68 & 34 & $W 158$ & 42 & $7 C 5 B+1 A 3$ \\
\hline PB & $Z N C U A G A U$ & & & & & N59 & 38 & $w 154$ & 01 & $70456 \mathrm{C} 3 \mathrm{~A}$ \\
\hline AU & & & & & & N58 & 31 & $w 134$ & 47 & 4A45C7A5 \\
\hline SN & & $u$ & $\mathrm{CU}$ & HG & & N65 & 56 & $w 166$ & 13 & $1 B 14 C 4 A 8$ \\
\hline$C R$ & & & & & & N6 1 & 27 & $W 149$ & 08 & $5 A 58 C$ \\
\hline $\mathrm{cu}$ & & $A U$ & $A G$ & PB & $\mathrm{ZN}$ & N6O & 54 & $W 146$ & 41 & $3 A 5 C 11 A 6$ \\
\hline$A U$ & & & & & & N67 & 20 & $w 150$ & 12 & $4 A 3 C 4 A B$ \\
\hline AU & & SB & $A G$ & & & N64 & 52 & $W 148$ & 03* & $4 A 145 C 7 B 5$ \\
\hline AU & & & & & & N65 & 04 & $w 147$ & 11 & $4 A 3 C 4 A B$ \\
\hline$A U$ & & $\mathbf{A G}$ & SB & SN & W BI & N64 & 57 & $W 147$ & $50 *$ & 4АЗB 4B8 \\
\hline$A U$ & & $H G$ & & & & N62 & 04 & $w 160$ & 59 & $\triangle A 3 C 4 A 8$ \\
\hline
\end{tabular}




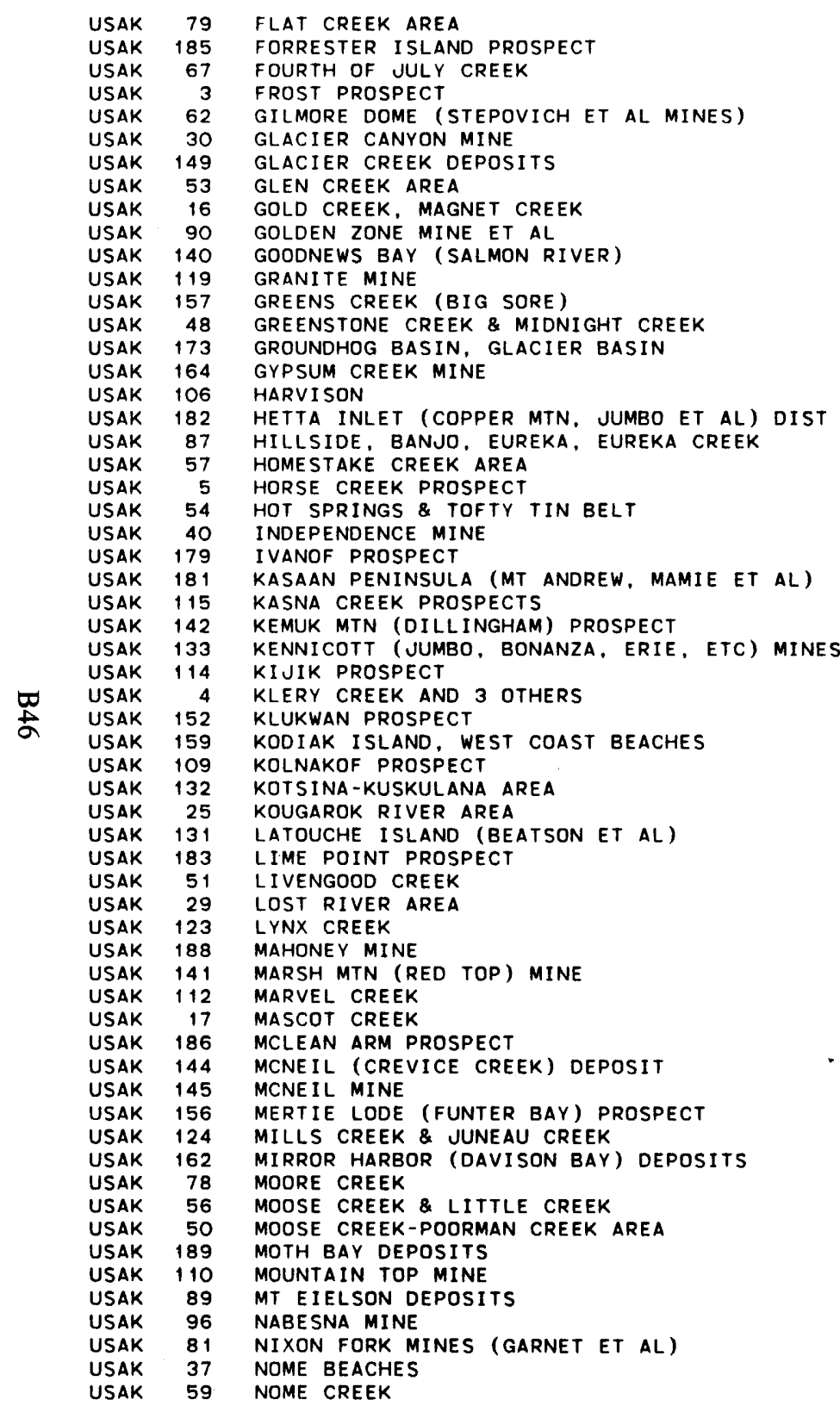

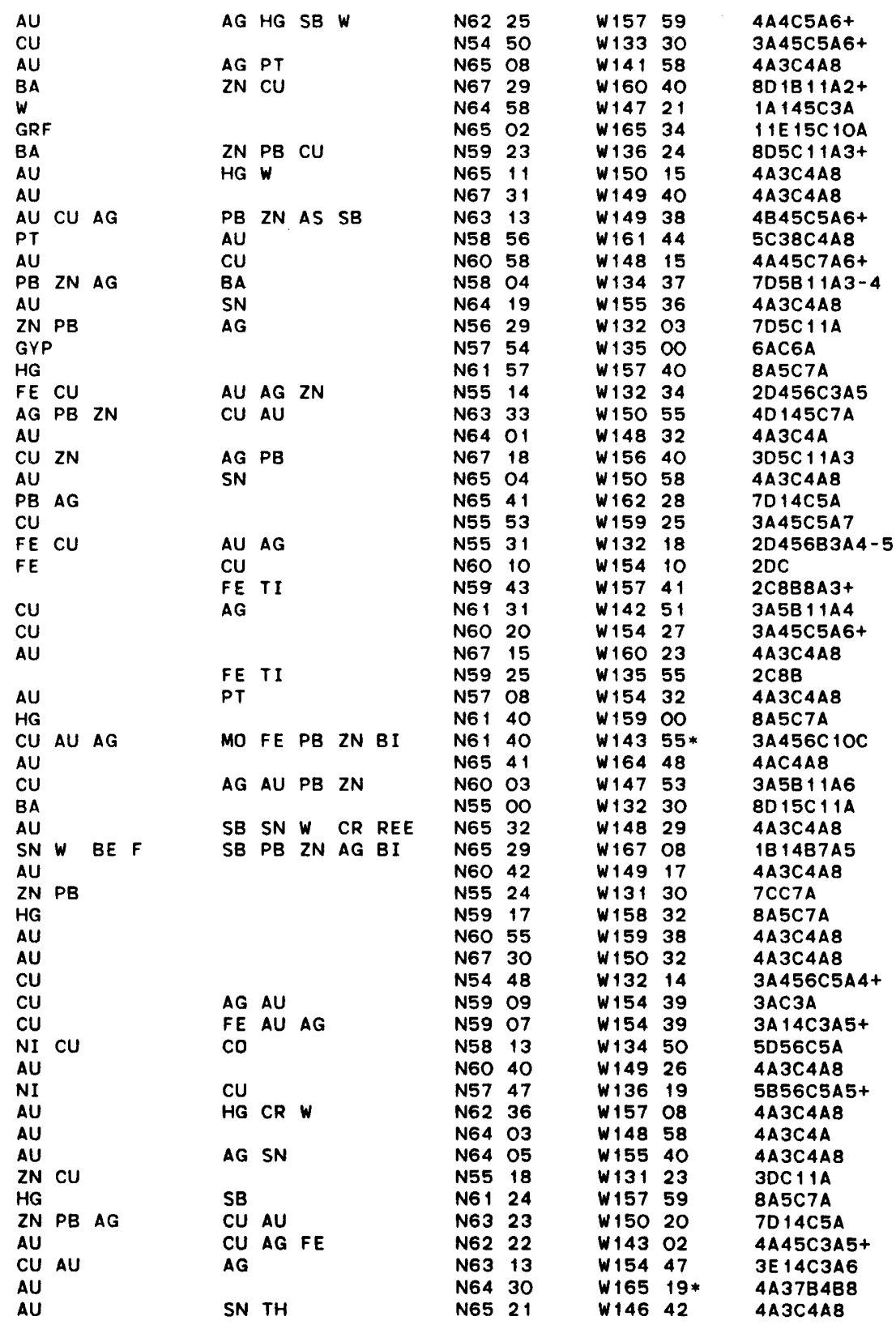




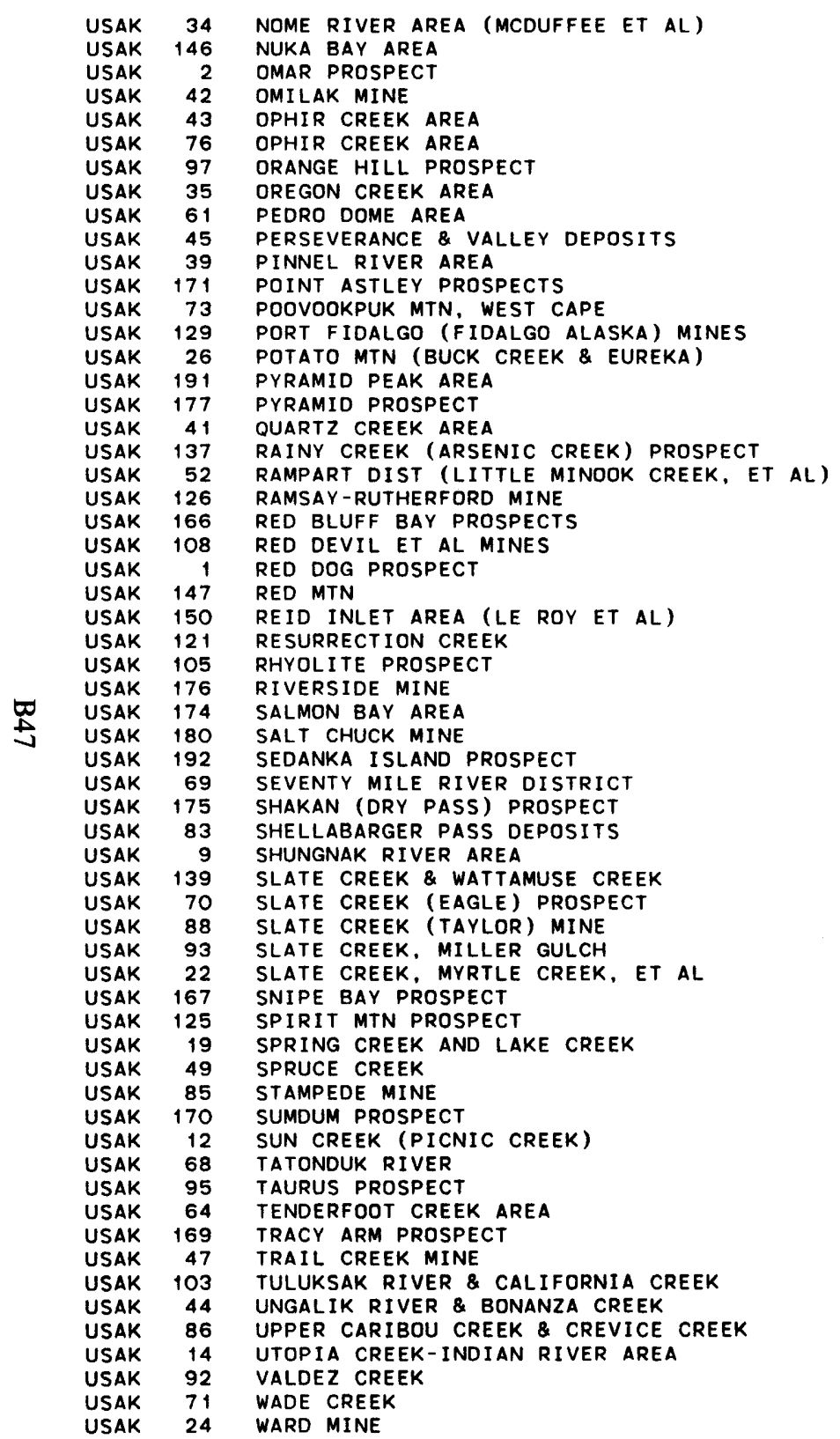

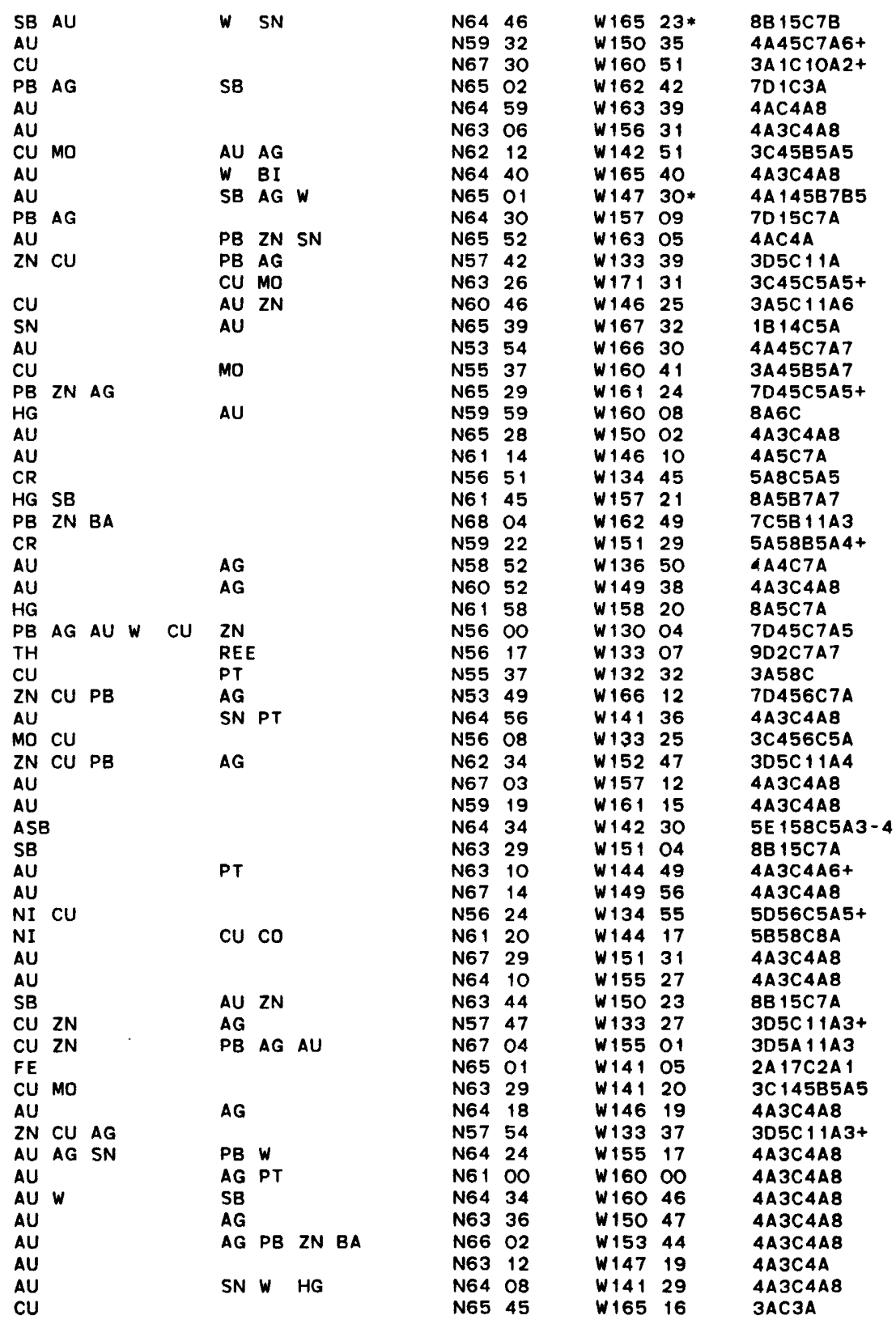




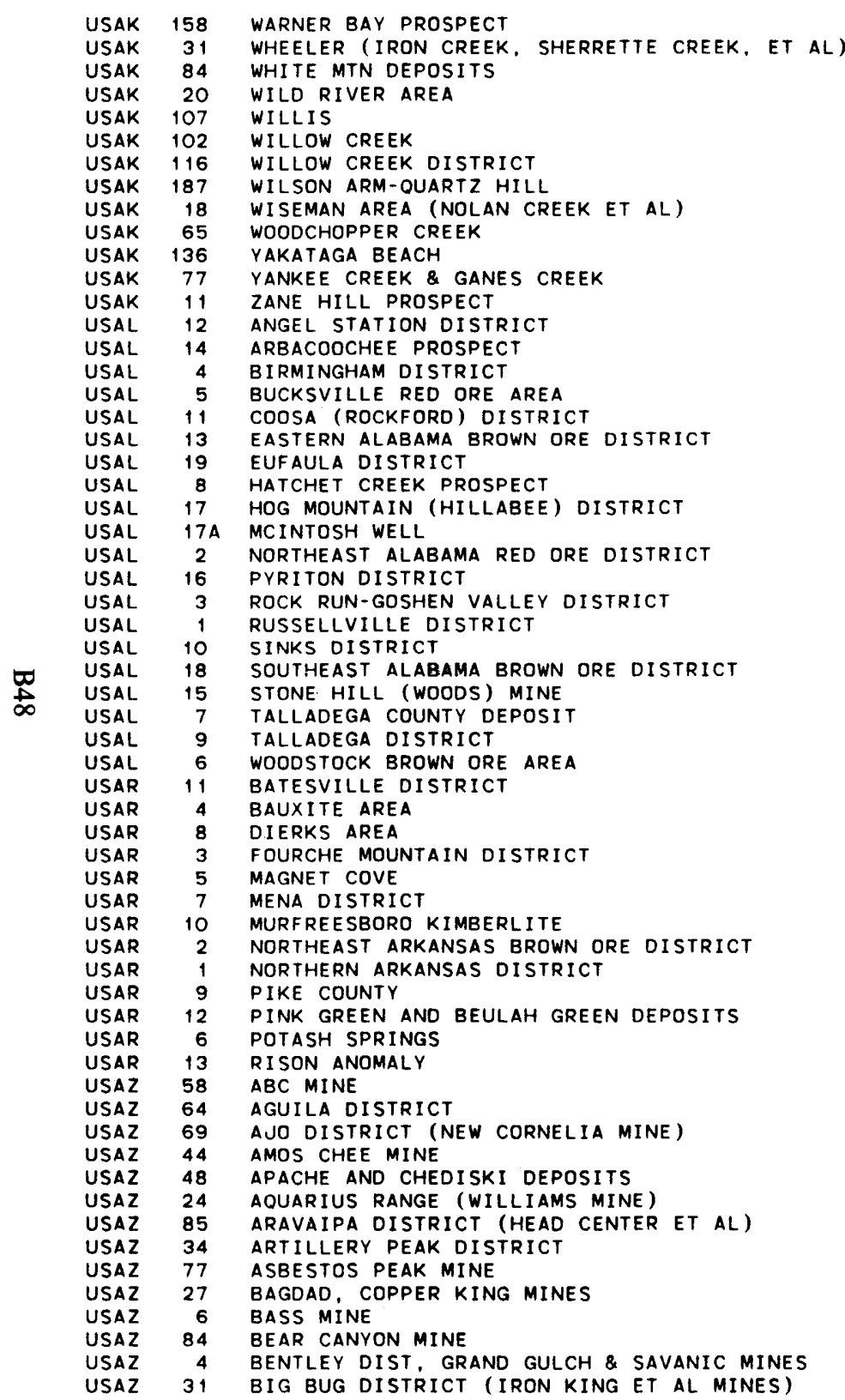

\begin{tabular}{|c|c|c|c|c|c|c|c|c|c|c|c|}
\hline $\mathrm{CU}$ & & & MO & $A G$ & $A U$ & PB ZN & N56 & 10 & W158 & 25 & 3A47C5A6t \\
\hline $\mathrm{cu}$ & PB & AG & & & & & N64 & 55 & $W 164$ & 42 & $3 A C$ \\
\hline HG & & & & & & & N62 & 13 & W154 & 58 & $8 A 1 C 7 A 7$ \\
\hline$A U$ & & & & & & & N67 & 26 & $W 151$ & 15 & $4 A 3 C 4 A 8$ \\
\hline HG & & & SB & & & & N6 1 & 50 & W157 & 22 & $8 A 5 C 7 A$ \\
\hline$A U$ & & & PT & & & & N61 & 50 & $W 161$ & 54 & $4 A 3 C 4 A 8$ \\
\hline$A U$ & & & $\mathrm{CU}$ & TE & $\mathbf{w}$ & PB ZN & N6 1 & 47 & $W 149$ & 19 & 4A 4B7A6 \\
\hline мо & & & & & & & N55 & 30 & $W 130$ & 35 & 3B4B5A 7 \\
\hline$A U$ & & & & & & & N67 & 30 & $W 150$ & 13 & $4 A 3 C 4 A 8$ \\
\hline$A U$ & & & PT & $\mathrm{TH}$ & & & N65 & 18 & $W 143$ & 24 & $\triangle A 3 C 4 A B$ \\
\hline$A U$ & & & $A G$ & & & & N60 & 03 & $W 142$ & 12 & $\triangle A 37 C 4 A B$ \\
\hline$A U$ & & & & & & & N63 & $\infty$ & $W 156$ & 26 & $4 A 3 C 4 A B$ \\
\hline CU & & & & & & & N66 & 20 & W156 & 05 & $3 A 45 C 5 A 5+$ \\
\hline BA & & & & & & & N33 & 52 & W085 & 50 & 8D1C10A4t \\
\hline$A U$ & & & & & & & N33 & 34 & WO85 & 33 & $4 A 15 C 7 A 3+$ \\
\hline FE & & & & & & & N33 & 29 & WOB6 & 46 & 2A1A2A4 \\
\hline $\mathrm{FE}$ & & & & & & & N33 & 10 & W087 & $10 *$ & $2 A 1 C 2 B 4$ \\
\hline & & & SN & & & & N32 & 58 & W086 & $09 *$ & $18145 C 183+$ \\
\hline FE & & & & & & & N33 & 55 & W085 & $57 *$ & $2 A 1 C 9 C 7+$ \\
\hline$A L$ & & & TI & & & & N31 & 45 & WOB6 & 16 & 10B37C9A7+ \\
\hline & & & $\mathrm{Cu}$ & мо & & & N33 & 17 & W086 & 02 & $3 \mathrm{C} 15 \mathrm{C}$ \\
\hline$A U$ & & & $\mathrm{CU}$ & BI & & & N33 & 05 & W085 & 48 & 4B 145C7A3+ \\
\hline HAL & & & & & & & N31 & 32 & W088 & 05 & $683 B 6 \times 5$ \\
\hline $\mathrm{FE}$ & & & & & & & N34 & 22 & W085 & $52 *$ & $2 A 1 C 2 B 4$ \\
\hline FES & & & CU & $\mathrm{ZN}$ & & & N33 & 21 & W085 & 49 & $3015 \mathrm{C} 11 \mathrm{A3}+$ \\
\hline$A L$ & & & & & & & N34 & 02 & W085 & $37 *$ & 1OB 1C9B8 \\
\hline $\mathrm{FE}$ & & & & & & & N34 & 30 & WO87 & $48 *$ & 2A37B9B4+ \\
\hline BA & & & & & & & N33 & 02 & W087 & or & BD 1C1OA4t \\
\hline FE & & & MN & & & & N31 & 48 & W085 & $10 *$ & $2 A 37 B 9 B 7+$ \\
\hline $\mathrm{cu}$ & & & $2 \mathrm{~N}$ & & & & N33 & 29 & W085 & 27 & $3015 C 11 A 3+$ \\
\hline TLC & & & & & & & N33 & 19 & W086 & 13 & 1OE 1C $11 A 3+$ \\
\hline $\mathrm{FE}$ & & & & & & & N33 & 11 & W086 & 13 & $2 A 15 C 4 A 3$ \\
\hline FE & & & & & & & N33 & 13 & W086 & 57 & 2A1C9A4t \\
\hline MN & & & & & & & N35 & 52 & W091 & $44 *$ & 1OA37B 1OB3+ \\
\hline$A L$ & & & & & & & N34 & 31 & W092 & 30 & 1OB2B9A7 \\
\hline BA & & & & & & & N34 & 09 & W093 & 57 & $8 D 37 B 10 A$ \\
\hline$A L$ & & & & & & & N34 & 40 & W092 & 16 & 1OB2B9A7 \\
\hline BA & & & & & & & N34 & 28 & WO92 & 49 & BDA $11 A 4$ \\
\hline MN & & & & & & & N34 & 23 & W093 & 44* & 1OAC2B3 \\
\hline DIA & & & & & & & N34 & 03 & W093 & 42 & $11028 C 5 A 6$ \\
\hline FE & & & & & & & N56 & 20 & w090 & 25* & $2 A 37 \mathrm{C} 9 \mathrm{C}$ \\
\hline $\mathrm{ZN}$ & PB & & $C D$ & $\mathrm{CU}$ & & & N36 & 12 & W092 & 39* & $7 C 37 C 1084+$ \\
\hline HG & & & & & & & N34 & 09 & W093 & 37 & $8 A C 7$ \\
\hline TI & & & & & & & N33 & 53 & W093 & 56 & $2 B 37 \mathrm{C} 4 \mathrm{~A} 6$ \\
\hline$v$ & & & & & & & N34 & 27 & W092 & 57 & 9B28B5A6 \\
\hline FE & & & & & & & N33 & 57 & W092 & 12 & 2A28C5AG \\
\hline MN & & & & & & & N33 & 13 & $W 114$ & 37 & 10A7C7A7 \\
\hline MN & & & & & & & N33 & 43 & $W 113$ & $09 *$ & 10A7C7B 7 \\
\hline $\mathrm{Cu}$ & $\mathrm{AU}$ & AG & $\mathrm{ZN}$ & мо & & & N32 & 21 & $W 112$ & 52 & 3A4A5A6 \\
\hline$u$ & & & & & & & N35 & 31 & $W 111$ & 05 & 9A37C1OA5+ \\
\hline FE & & & & & & & N34 & 05 & W110 & 42 & 2A37B2A1 \\
\hline w & & & & & & & N34 & 47 & $W 113$ & 26 & 1AC7A \\
\hline & $Z N$ & CU AG AU & & & & & N32 & 58 & $W 110$ & 20 & $70347 C 7 A 6+$ \\
\hline MN & & & & & & & N34 & 18 & $W 113$ & 36 & 1OA3A 1OA7 \\
\hline ASB & & & & & & & N33 & 46 & $W 110$ & 57 & 5E $367 C$ 1OA1 \\
\hline CU & & & $A U$ & $A G$ & PB & $Z N$ & N34 & 35 & W113 & 12 & 3C4B5A6 \\
\hline ASB & & & & & & & N36 & 13 & $W 112$ & 22 & SE $367 C_{10 A 2}$ \\
\hline ASB & & & & & & & N33 & 30 & $W 110$ & 22 & SE $367 C_{1 O A 1}$ \\
\hline $\mathrm{Cu}$ & & & & & & & N36 & 16 & $W 113$ & 48 & $3 A 37 C 5 A 4+$ \\
\hline $2 \mathrm{~N}$ & PB & CU AG AU & & & & & N34 & 29 & $W 112$ & 16 & 7D5B $11 \mathrm{~A} 1$ \\
\hline
\end{tabular}




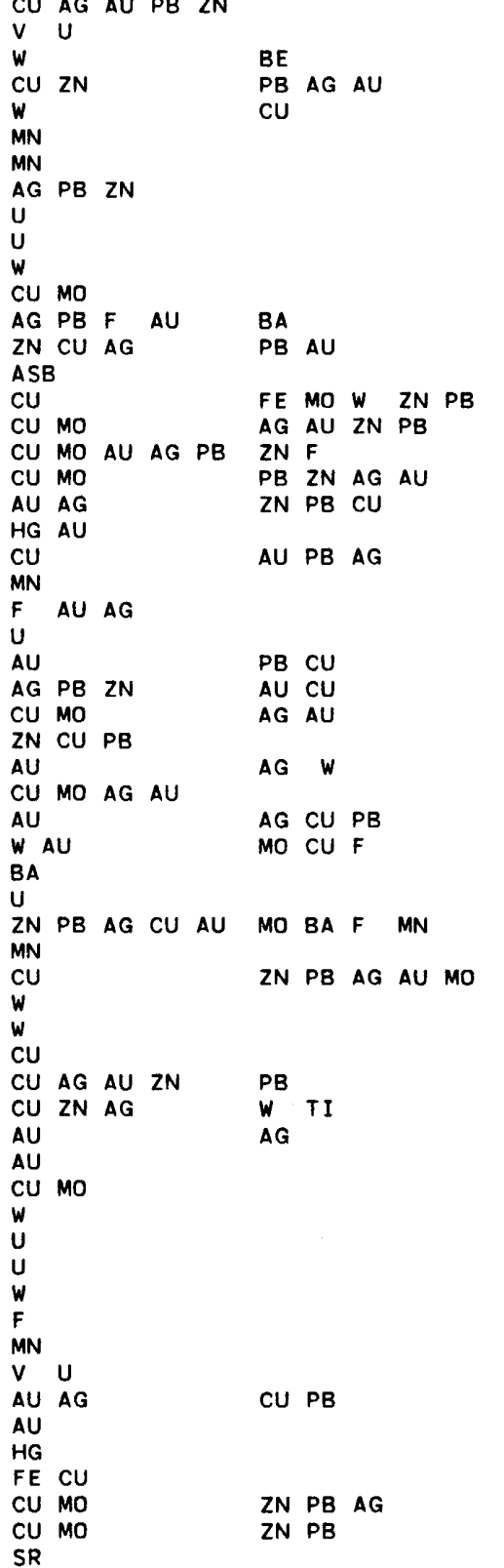
N36 17 $\begin{array}{lll}W 113 & 02 \\ W 112 & 14\end{array}$ N34 $57 \quad W 11355$ N33 54 N33 54 N34 18 N31 59 $\begin{array}{lll}\text { N35 } & 19 \\ \text { N35 } & 45 \\ \text { N } 32 & 33\end{array}$ N35 45 N35 35 N32 52 N33 02 N34 52 N33 57 N33 04 N33 05 N34 29 N32 45 N34 10 N33 32 N32 13 N34 07 N32 50 N31 40 N33 38 N31 53 N31 53 N34 32 N32 32 N33 25 N35 55 $\begin{array}{ll}\text { N33 } & 55 \\ \text { N33 } & 33\end{array}$ N33 33 N36 35 N31 28 N34 20 N31 52 N31 26 N33 45 N36 40 N34 45 N32 07 N33 18 N33 38 N32 32 N31 40 N36 46 N32 1 N32 N31 51 N34 36 N36 31 N34 28 N34 12 N33 57 N34 14 N35 22 N31 59 N32 45
3D347A5A4 IA4C7A1 3D 15C1 1A 1 1A 145B7A 1OA7C7A7

1OA3C $11 A B$

4DC

W11400

W109 14

W110 58

$\begin{array}{lll}W & 20 \\ w & & \\ W & 41 & 44\end{array}$

$W 110$
$W 111$

W114 11

$\begin{array}{lll}W & 113 & 57\end{array}$

W110 52

$W 11046$

W109 22

$W 11235$

$W 11029$

W112 20

$W 11419$

W109 36

$W 11340$

W109 06

$W+1102$

$W 11330$

$\begin{array}{lll}W 110 & 36 \\ W & 11 & \end{array}$

$\begin{array}{lll}W & 08 \\ W & 113 & 13\end{array}$

$W 113$
$W 114$
$W$

$\begin{array}{lll}W & 114 & 18 \\ W & 110 & 50\end{array}$

Wi1 50

$W 11409$

W111 39

$\begin{array}{lll}W 112 & 47\end{array}$

$W 11044$

$W+1045$

$W 11047$

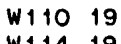

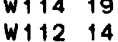

W112 07

W110 04

$W 11358$

$W 11424$
$W 111$

W111 52

$\begin{array}{ll}W 111 & 23 \\ w & 111 \\ w 3 *\end{array}$

W111 43*

$W 11209$

$W 11008$
$W 11023$

$w 11120$

W109 13*

$W 11221$

$W 11250$

W111 26

$W 11400$

W114 09

W111 04

$\begin{array}{ll}W & 11247\end{array}$

A 77 C $10 A 5+$

$9 A 37 B 10 B 5+$

$1 A 4 C 3 A$

3C 145A5A6

4D4C7A6+

3D 15C11A1
5E $367 C$ 1OA

3A347B3A6

3C4A5A6

3C 145B5A6

3C4C5A6

4C 145C7A6+

8A 15C7A

3A347C3A6

10A3C 1OA

9A3C7AG

AAC7A

40347C5A6+

4D347C5A

3C4A5AG

4A 15C7A

4A 15C7A

3C4A5A6

4A 125C7A

1A4C7A

8DB7A

9A37C5A5+

7D347B7A

10AC9A

3А $347 B 3 A 6$

1AC7A

$1 A C$

3A37C 10A4+

3D5A11A1

3D347B3A6

4A7C7A7

4AC $4 A 8$

3C4A5A

9A4C7A

9A37C10B5+

9A3C7A6+

1A4C7A6

8C 15C7A

10A37C9A
9 C $37 B 10 B 5+$

4C 145C7A

4AC7A

8A 15C7A

2D 14C3A

3C $145 B 5 A 6$

3C347A3A
8EC6A6+ 


\begin{tabular}{|c|c|c|}
\hline USAZ & 12 & MONUMENT NO 2 MINE \\
\hline USAZ & 10 & MONUMENT VALLEY AREA (MOONLIGHT MINE) \\
\hline USAZ & 49 & MORALE CLAIM \\
\hline USAZ & 13 & NORTHWEST CARRIZO MTS AREA \\
\hline USAZ & 90 & OLD HAT DISTRICT \\
\hline USAZ & 87 & OMEGA MINE \\
\hline USAZ & 107 & ORO BLANCO DISTRICT (MONTANA MINE) \\
\hline USAZ & 7 & ORPHAN LODE MINE \\
\hline USAZ & 110 & PATAGONIA DIST (DUQUESNE, MOWRY MINES) \\
\hline USAZ & 40 & PECK DISTRICT (DE SOTO ET AL MINES) \\
\hline USAZ & 67 & PIKES PEAK DISTRICT \\
\hline USAZ & 33 & PLANET DISTRICT (SWANSEA, CLARA, PLANET) \\
\hline USAZ & 79 & POSTON BUTTE \\
\hline USAZ & 1 & RADON GROUP \\
\hline USAZ & 82 & RAY DISTRICT (RAY \& COPPER BUTTE MINES) \\
\hline USAZ & 5 & RIDENOUR MINE \\
\hline USAZ & 50 & ROCK GARDEN ET AL CLAIMS \\
\hline USAZ & $86 \mathrm{~A}$ & SACATON \\
\hline USAZ & 95 & SAFFORD DEPOSIT (LONE STAR DISTRICT) \\
\hline USAZ & 78 & SALT RIVER CHRYSOTILE AREA \\
\hline USAZ & 21 & SAN FRANCISCO (OATMAN) DISTRICT \\
\hline USAZ & 88 & SAN MANUEL-KALAMAZOO DEPOSIT \\
\hline USAZ & 59 & SILVER (RED CLOUD, BLACK ROCK) DISTRICT \\
\hline USAZ & 92 & SILVER BELL \\
\hline USAZ & 53 & ST JOHNS AREA \\
\hline USAZ & 51 & SUPAI POTASH BEDS \\
\hline USAZ & 52 & SUPAI SALT BASIN \\
\hline USAZ & 81 & SUPERIOR (PIONEER) DISTRICT (MAGMA MINE) \\
\hline USAZ & 115 & SWISSHELM DISTRICT \\
\hline USAZ & $\$ 12$ & TOMBSTONE DISTRICT \\
\hline USAZ & 113 & TURQUOISE DISTRICT \\
\hline USAZ & 102 & TWIN BUTTES MINE \\
\hline USAZ & 108 & TYNDALL DISTRICT \\
\hline USAZ & 20 & UNION PASS DIST (KATHERINE MINE) \\
\hline USAZ & 36 & URANIUM AIRE GROUP \\
\hline USAZ & 70 & VEKOL HILLS (REWARD MINE) \\
\hline USAZ & 66 & VULTURE MINE \\
\hline USAZ & 65 & VULTURE MTS DISTRICT \\
\hline USAZ & 18 & WALLAPAI DIST (CHLORIDE \& CERBAT CAMPS) \\
\hline USAZ & 17 & WEAVER DISTRICT \\
\hline USAZ & 38 & WEAVER-RICH HILL DISTRICT \\
\hline USAZ & 9 & WHITE MESA (KAIBITO PLATEAU) \\
\hline USAZ & 76 & WORKMAN CREEK (SIERRA ANCHA) AREA \\
\hline USCA & 176 & AGUANGA DISTRICT \\
\hline USCA & 49 & ALLEGHANY-DOWNIEVILLE DISTRICT \\
\hline USCA & 63 & ALPINE MINE \\
\hline USCA & 14 & ALTOONA \\
\hline USCA & 152 & ATOLIA DISTRICT \\
\hline USCA & $119 A$ & BALLARAT DISTRICT (RATCLIFF MINE) \\
\hline USCA & 62 & BELLOTA (CALVERAS RIVER) DISTRICTS \\
\hline USCA & 24 & BENSON GROUP \\
\hline USCA & 96 & BLACK CANYON DISTRICT \\
\hline USCA & 32 & BLACK DIAMOND (GREY EAGLE) GROUP \\
\hline USCA & 91 & BLACK ROCK MINE \\
\hline USCA & 90 & BLIND SPRING DISTRICT \\
\hline USCA & 27 & BLUE JAY MINE \\
\hline USCA & 69 & BODIE DISTRICT \\
\hline USCA & 52 & BOILER PIT \\
\hline USCA & 15 & BOULDER CREEK MINE \\
\hline USCA & 30 & BRAITO MINE \\
\hline
\end{tabular}

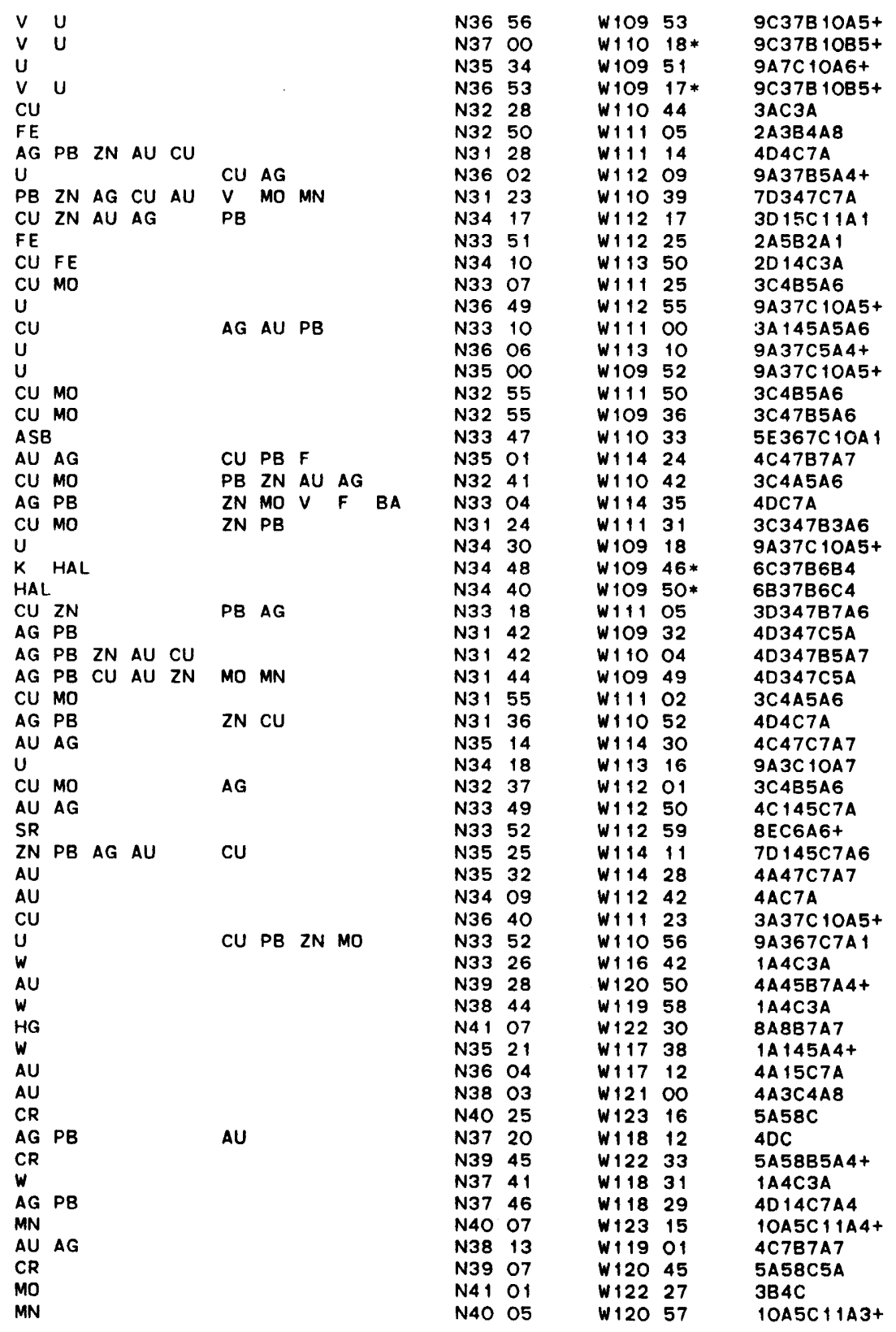




\begin{tabular}{|c|c|c|}
\hline $\begin{array}{l}\text { USCA } \\
\text { USCA }\end{array}$ & $\begin{array}{l}79 \\
88\end{array}$ & $\begin{array}{l}\text { BUCKEYE MINE } \\
\text { BUTLER ESTATE (MISTAKE) }\end{array}$ \\
\hline USCA & 140 & CACHUMA \\
\hline USCA & 158 & CALICO BORATES \\
\hline USCA & 157 & CALICO DISTRICT \\
\hline USCA & 187 & CARGO MUCHACHO DISTRICT \\
\hline USCA & 159 & CAVE CANYON \\
\hline USCA & 129 & CEDAR CREEK DISTRICT \\
\hline USCA & 85 & CENTRAL SAN BENITO \\
\hline USCA & 110 & CERRO GORDO DISTRICT \\
\hline USCA & $111 \mathrm{~A}$ & CHLORIDE CLIFF DISTRICT \\
\hline USCA & 103 & CLARA H LACEY \& JACK SPRATT MINES \\
\hline USCA & 34 & CLEAR LAKE DIST (SULPHUR BANK MINE) \\
\hline USCA & 171 & CLIFTON AREA \\
\hline USCA & 87 & COALINGA AREA (VARIOUS MINES) \\
\hline USCA & 135 & COAST RANGE PHOSPHATE AREA \\
\hline USCA & 13 & COGGINS \& LITTLE CASTLE CREEK \\
\hline USCA & 106 & CONSOLIDATED TUNGSTEN (HARREL HILL) MINE \\
\hline USCA & $102 A$ & COPPER KING \\
\hline USCA & 72 & COPPEROPOLIS (PACIFIC ASBESTOS) MINE \\
\hline USCA & 71 & COPPEROPOL IS DISTRICT (COPPER KING MINE) \\
\hline USCA & 120 & coso \\
\hline USCA & 175 & COTTONWOOD \\
\hline USCA & 130 & COVE DISTRICT \\
\hline USCA & 21 & COW CREEK DISTRICT (INGOT, AFTERTHOUGHT) \\
\hline USCA & 2 & CRESCENT CITY BEACH SANDS \\
\hline USCA & $30 \mathrm{~A}$ & CRESCENT HILLS DISTRICT (GREEN MTN, ET AL) \\
\hline USCA & 53 & DAIRY FARM (VALLEY VIEW) DEPOSITS \\
\hline USCA & 114 & DARWIN (COSO) DISTRICT \\
\hline USCA & 144 & DEATH VALLEY TALC MINES \\
\hline USCA & 3 & DEL NORTE COUNTY CHROMITE DISTRICT \\
\hline USCA & 101 & DINKEY CREEK (LOBO, SADDLER) MINE \\
\hline USCA & 54 & DOBBAS-PILLIKEN DEPOSITS \\
\hline USCA & 181 & EAGLE MOUNTAIN DISTRICT \\
\hline USCA & 20 & EAST SHASTA DISTRICT \\
\hline USCA & 7 & FAIRVIEW \\
\hline USCA & 180 & FISH CREEK MINE \\
\hline USCA & 56 & FOLSOM DISTRICT \\
\hline USCA & 61 & FOOTHILL BELT DISTRICT (PENN MINE ET AL) \\
\hline USCA & 25 & FORT SEWARD MINE \\
\hline USCA & 155 & FOUR CORNERS \\
\hline USCA & 137 & FRAZIER MOUNTAIN \\
\hline USCA & 17 & FRENCH GULCH-DEADWOOD DISTRICT \\
\hline USCA & 118 & FURNACE CREEK GROUP \\
\hline USCA & 104 & GARNET DIKE \& QUIGLEY KINGS RIVER MINES \\
\hline USCA & 10 & GAZELLE MOUNTAIN DEPOSITS \\
\hline USCA & 40 & GENESEE DISTRICT (WALKER MINE) \\
\hline USCA & 58 & GEORGETOWN \& GRIZZLY FLAT \\
\hline USCA & 138 & GORMAN (MEEKE - HOGAN) DISTRICT \\
\hline USCA & 51 & GRASS VALLEY-NEVADA CITY DISTRICT \\
\hline USCA & 28 & GRAU MINE ET AL \\
\hline USCA & 6 & GRAY EAGLE (HAPPY CAMP DISTRICT) \\
\hline USCA & 99 & GRUB GULCH-HILDRETH DISTRICT \\
\hline USCA & 37 & GUERNEVILLE DISTRICT (SONOMA MINE) \\
\hline USCA & 42 & HALLELUJAH JUNCTION AREA \\
\hline USCA & 50 & HAMMONTON (YUBA RIVER) DISTRICT \\
\hline USCA & 23 & HARRISON GULCH DISTRICT (MIDAS MINE) \\
\hline USCA & 28A & HAYDEN HILL DISTRICT \\
\hline USCA & 146 & HI PEAK MINE \\
\hline USCA & 92 & HITES COVE DISTRICT (EAST BELT) \\
\hline
\end{tabular}

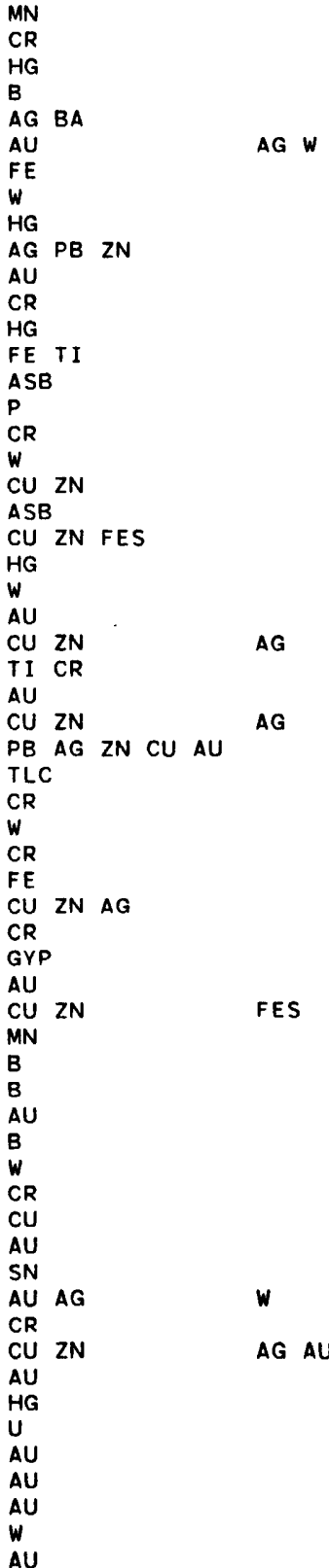

\begin{tabular}{|c|c|c|c|c|}
\hline $\begin{array}{l}\text { N37 } \\
\text { N36 }\end{array}$ & $\begin{array}{l}30 \\
19\end{array}$ & $\begin{array}{l}W 121 \\
W 120\end{array}$ & $\begin{array}{l}22 \\
30\end{array}$ & $\begin{array}{l}\text { 1OA5C } 11 A 4+ \\
5 A C\end{array}$ \\
\hline N34 & 43 & $w 119$ & 53 & $8 A C$ \\
\hline N34 & 56 & $W 116$ & 52 & 6E 3C6A7 \\
\hline N34 & 58 & $W 116$ & 49 & 4D7C7A7 \\
\hline N32 & 53 & $w 114$ & 49 & 4A 15C7A \\
\hline N35 & 04 & $W 116$ & 20 & $2 A 15 C$ \\
\hline N35 & 45 & $W 118$ & 34 & 1А4СЗА \\
\hline N36 & 37 & $W 121$ & 00 & $8 A C$ \\
\hline N36 & 32 & $W 117$ & 48 & 4D 14B5A5 \\
\hline N36 & 40 & $W 116$ & 55 & 4A 1C7A \\
\hline N36 & 54 & $W 119$ & 19 & $5 A 58 C 5 A$ \\
\hline N39 & $\infty$ & $W 122$ & 41 & 8AB7A8 \\
\hline N33 & 52 & $W 118$ & 23 & $2 C 37 C 4 A 8$ \\
\hline N36 & 19 & $W 120$ & 42 & 5E8A5A \\
\hline N34 & 55 & $W 120$ & OO* & $2 \mathrm{E} 1 \mathrm{C} 2 \mathrm{~B} 7$ \\
\hline N41 & 12 & $W 122$ & 17 & $5 A 58 C$ \\
\hline N36 & 38 & $w 119$ & 07 & 1АCЗА \\
\hline N36 & 55 & $W 119$ & 27 & $3 D 5 C+1 A 4$ \\
\hline N37 & 57 & $W 120$ & 32 & 5E8B5A4 \\
\hline N37 & 59 & $W 120$ & 39 & $305 B 11 A 4$ \\
\hline N36 & 01 & W117 & 47 & BAC \\
\hline N33 & 44 & W116 & 27 & 1А4С $3 A$ \\
\hline N35 & 44 & $W 118$ & 26 & 4AC7A \\
\hline $\mathrm{N} 4 \mathrm{O}$ & 43 & W122 & 04 & $3 D 5 C 11 A 4+$ \\
\hline N41 & 44 & $W 124$ & 09 & $2 C 37 C 4 A 8$ \\
\hline N4O & 05 & $W 120$ & 52 & 4A45C7A4 \\
\hline N39 & 00 & $W 121$ & 18 & $305 C 11 A$ \\
\hline N36 & 16 & W117 & 33 & 7D4B3A5 \\
\hline N36 & 00 & $w 116$ & 55 & 1OE $16 \mathrm{C} 11 \mathrm{~A}$ \\
\hline N41 & 48 & W123 & $54 *$ & 5A58B5B \\
\hline N37 & 03 & W119 & 09 & $1 A 4 C$ \\
\hline N38 & 47 & $W 121$ & 06 & 5A58B5A \\
\hline N33 & 55 & W115 & 35 & 2A14A3A4+ \\
\hline $\mathrm{N} 4 \mathrm{O}$ & 48 & $W 122$ & 12 & $305 C 11 A 4+$ \\
\hline N41 & 49 & $w 123$ & 07 & $5 A C$ \\
\hline N33 & 04 & W116 & 06 & 6A37B6A7 \\
\hline N38 & 38 & $W 121$ & 13 & AA3B $4 A 8$ \\
\hline N38 & 13 & $W 120$ & $51 *$ & $305 B 11 B 4$ \\
\hline N4O & 12 & W123 & 39 & 1OA5C $11 A 4$ \\
\hline N35 & 01 & W117 & 28 & 6E 3B6A7 7 \\
\hline N34 & 47 & $W 119$ & 04 & 6E 3C6A7 \\
\hline N4O & 42 & $W 122$ & 37 & 4A45C7A \\
\hline N36 & 18 & $W 115$ & $36 *$ & $6 E 3 B 6 B 7$ \\
\hline N36 & 53 & $W 119$ & 01 & $1 A 4 C$ \\
\hline N41 & 25 & W122 & 32 & 5A58C5A \\
\hline N39 & 58 & $W 120$ & 40 & 3A45B7A4+ \\
\hline N38 & 39 & $W 120$ & 29 & $4 A 3 C 4 A 6+$ \\
\hline N34 & 50 & $W 118$ & 43 & 1B4C3A \\
\hline N39 & 14 & $W 121$ & 03 & 4A45A7A4+ \\
\hline N4O & 02 & $W 123$ & 40 & 5A58B5A4+ \\
\hline N4 1 & 53 & $W 123$ & 22 & 3D5C 10A \\
\hline N37 & 12 & $W 119$ & 37 & $4 A C$ \\
\hline N38 & 34 & $W 122$ & 59 & 8A5B7A7+ \\
\hline N39 & 52 & $W 120$ & 01 & 9A4C7A \\
\hline N39 & 12 & $w 121$ & 26 & $\triangle A 3 B 4 A B$ \\
\hline N4O & 25 & $w 122$ & 55 & 4A3C7A \\
\hline N41 & $\infty$ & $W 120$ & 40 & 4A7C7A7 \\
\hline N35 & 41 & W117 & 53 & 1A14C3A \\
\hline N37 & 31 & $W 119$ & 58 & 4A45C7A \\
\hline
\end{tabular}




\begin{tabular}{|c|c|c|}
\hline USCA & $163 A$ & HOLCOMB DISTRICT \\
\hline USCA & 109 & HOLSTON \\
\hline USCA & 22 & IGO DISTRICT \\
\hline USCA & 170 & IRON AGE \\
\hline USCA & 169 & IRON HAT (IRONCLAD) \\
\hline USCA & 153 & IRON MTN-IRON KING \\
\hline USCA & 183 & IRONWOOD DISTRICT (BLACKJACK AND LANGDON MINES) \\
\hline USCA & 26 & ISLAND MTN DISTRICT \\
\hline USCA & 102 & JACKPOT, BENSON-MCMURTRY AND OTHER MINES \\
\hline USCA & 46 & JOHNSVILLE DISTRICT (PLUMAS EUREKA DISTRICT) \\
\hline USCA & 178 & JULIAN DISTRICT \\
\hline USCA & 67 & JUNIPER MINE \\
\hline USCA & 164 & KINGSTON DISTRICT (BECK DEPOSIT) \\
\hline USCA & 149 & KINGSTON RANGE DIST (SHEEP CREEK ET AL MINES) \\
\hline USCA & 8 & KLAMATH RIVER DISTRICT \\
\hline USCA & 124 & KLAU MINE \\
\hline USCA & 35 & KNOXVILLE DISTRICT \\
\hline USCA & 39 & KOHLER \& CHASE (NAPA) MINE \\
\hline USCA & 154 & KRAMER \\
\hline USCA & 78 & LADD MINE \\
\hline USCA & 43 & LAMBERT \\
\hline USCA & 142 & LANG \\
\hline USCA & 162 & LAVA BED DIST (MORRIS LODE, \\
\hline USCA & 29 & LIGHTS CANYON (ENGELS AND SUPERIOR MINES) \\
\hline USCA & 31 & LITTLE RED MTN \\
\hline USCA & 141 & LOS PRIETOS \\
\hline USCA & 131 & LOST HILLS MINE \\
\hline USCA & 44 & MAGAL IA \\
\hline USCA & 98 & MARBLE MINE \\
\hline USCA & 74 & MARSHS FLAT GROUP \\
\hline USCA & 68 & MASONIC DISTRICT (PITTSBURGH-LIBERTY MINE) \\
\hline USCA & 36 & MAYACMAS DIST, (OAT HILL ET AC) \\
\hline USCA & 9 & MCGUFFY CREEK \\
\hline USCA & 177 & MESA GRANDE (HIMALAYA) MINE \\
\hline USCA & 182 & MIDLAND (LITTLE MARIA MOUNTAINS) MINE \\
\hline USCA & 93 & MINARETS (IRON MTN) DEPOSIT \\
\hline USCA & 115 & MODOC DISTRICT \\
\hline USCA & 41 & MOHAWK, MURDOCK, JUMBO \\
\hline USCA & 65 & MONITOR DISTRICT \\
\hline USCA & 60 & MOTHER LODE DISTRICT, AMADOR, CALAVERAS COS. \\
\hline USCA & 55 & MOTHER LODE IN EL DORADO COUNTY \\
\hline USCA & 73 & MOTHER LODE-EAST BELT \\
\hline USCA & 75 & MOTHER LODE - WEST BELT \\
\hline USCA & 165 & MOUNTAIN PASS AREA \\
\hline USCA & 100 & MUD LAKE (GARNET) DEPOSIT \\
\hline USCA & 57 & MURPHY \\
\hline USCA & 83 & NEW ALMADEN DISTRICT \\
\hline USCA & 148 & NEW DEAL (OWL HOLE) \\
\hline USCA & 86 & NEW IDRIA \\
\hline USCA & 109A & NORTH PANAMINT RANGE MINES \\
\hline USCA & 81 & NUMBER 5 AND ADOBE CANYON \\
\hline USCA & 76 & OAKDALE-KNIGHTS FERRY DISTRICT \\
\hline USCA & 38 & OAKVILLE \\
\hline USCA & 105 & OBELISK MINE \\
\hline USCA & 123 & OCEANIC MINE \\
\hline USCA & 188 & OGILBY MINE \\
\hline USCA & 167 & OLD DAD MOUNTAIN \\
\hline USCA & 179 & OLD IRONSIDES (FRIDAY) MINE \\
\hline USCA & 185 & OROCOPIA \\
\hline USCA & 47 & OROVILLE DISTRICT \\
\hline
\end{tabular}

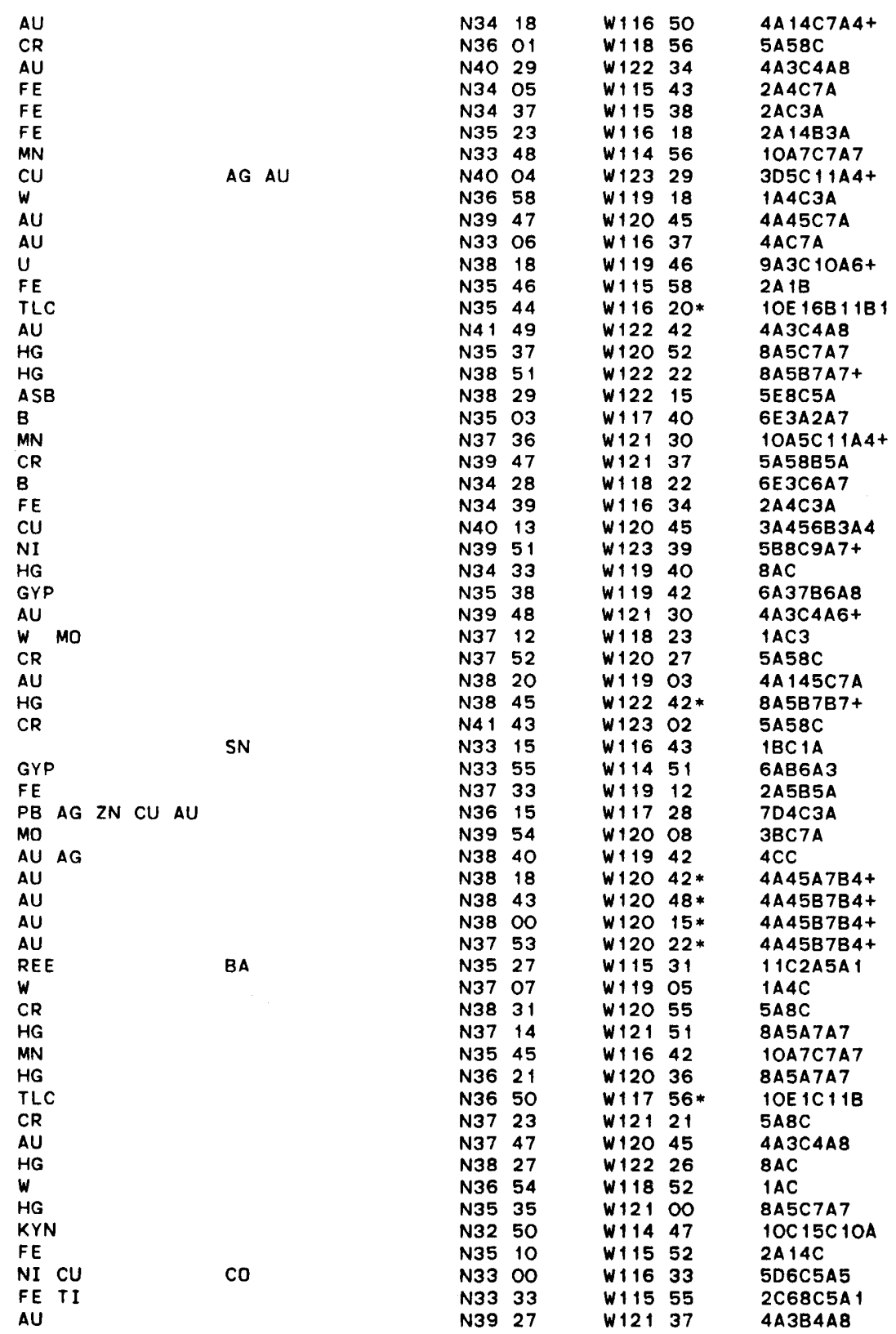




\begin{tabular}{|c|c|c|}
\hline USCA & 112 & OWENS LAKE \\
\hline USCA & $175 \mathrm{~A}$ & PALA DISTRICT (STEWART MINE) \\
\hline USCA & 119 & PANAMINT DISTRICT \\
\hline USCA & 122 & PARKFIELD \\
\hline USCA & 66 & PATTERSON DIST (SILVERADOR AND KENTUCK MINE \\
\hline USCA & 186 & PAYMASTER DISTRICT (WHEDON MINE) \\
\hline USCA & 174 & PINACATE DISTRICT \\
\hline USCA & 95 & PINE CREEK AREA \\
\hline USCA & 1 & PINE FLAT MOUNTAIN \\
\hline USCA & 107 & PIONEER, ROYAL TUNGSTEN, \& MARTIN CLAIMS \\
\hline USCA & 70 & QUAIL HILL \& NAPOLEON MINES \\
\hline USCA & 151 & RANDSBURG DISTRICT \\
\hline USCA & 150 & RESTING SPRINGS (TECOPA) DISTRICT \\
\hline USCA & 139 & ROSAMOND-MOJAVE DISTRICT \\
\hline USCA & 89 & SADDLEBACK LAKE \\
\hline USCA & 11 & SALMON RIVER DISTRICT \\
\hline USCA & 136 & SAN EMIGDIO (ANTIMONY PEAK) \\
\hline USCA & 143 & SAN GABRIEL MOUNTAINS \\
\hline USCA & 125 & SAN LUIS OBISPO DISTRICT \\
\hline USCA & 172 & SANTA CATALINA ISLAND \\
\hline USCA & 94 & SCHEELORE \\
\hline USCA & 97 & SCHOBER, OOMPH, BLACK MONSTER ET AL MINES \\
\hline USCA & 147 & SEARLES LAKE \\
\hline USCA & 5 & SEIAD CREEK (MOUNTAIN VIEW) \\
\hline USCA & 160 & SHADOW MOUNTAINS-NORTHERN PART \\
\hline USCA & 161 & SHADOW MOUNTAINS-SOUTHERN PART \\
\hline USCA & 19 & SHASTA-CALIFORNIA \\
\hline USCA & 121 & SHOSHONE - GERSTLEY \\
\hline USCA & 166 & SILVER LAKE-YUCCA GROVE DISTRICT \\
\hline USCA & 45 & SLATE CREEK (LA PORTE) DISTRICT \\
\hline USCA & 59 & SLOUGHHOUSE DISTRICT \\
\hline USCA & 82 & SNELLING DISTRICT \\
\hline USCA & 184 & SOUTHERN MCCOY MOUNTAINS GROUP \\
\hline USCA & 4 & SQUAW CREEK DISTRICT (BLUE LEDGE MINE) \\
\hline USCA & 156 & STARBR I GHT \\
\hline USCA & 84 & STAYTON DISTRICT (TRENCH, QUIEN SABE MINES) \\
\hline USCA & 163 & STEDMAN DIST (BAGDAD-CHASE, BUCKEYE ET AL) \\
\hline USCA & 113 & TALC CITY ET AL MINES \\
\hline USCA & 134 & TEHACHAPI \\
\hline USCA & 173 & TEMESCAL (CAJALCO) MINE \\
\hline USCA & 33 & THOMAS (ROUND MTN) MINE \\
\hline USCA & 132 & TOM MOORE, ERSKINE CREEK \\
\hline USCA & 117 & TRANSPORTATION (OLD DEPENDABLE) \\
\hline USCA & 16 & TRINITY RIVER BASIN \\
\hline USCA & 108 & TULARE COUNTY TUNGSTEN MINE \\
\hline USCA & 133 & TUNGSTEN CHIEF MINE \\
\hline USCA & 127 & TUNGSTORE MINE \\
\hline USCA & 111 & UBEHEBE AREA \\
\hline USCA & 64 & VALPINE MINE (BURNSIDE LAKE DEPOSIT) \\
\hline USCA & 168 & VULCAN \\
\hline USCA & 77 & WATERFORD DISTRICT \\
\hline USCA & 126 & WELCH MINE \\
\hline USCA & 18 & WEST SHASTA DISTRICT (FLAT CK., IRON MTN) \\
\hline USCA & 80 & WESTERN MINE \\
\hline USCA & $91 \mathrm{~A}$ & WHITE MOUNTAINS MINE \\
\hline USCA & $111 \mathrm{~B}$ & WILD ROSE DISTRICT (SKIDOO MINE) \\
\hline USCA & 116 & WILDROSE CANYON \\
\hline USCA & 145 & WINGATE WASH \\
\hline USCA & 128 & WOODY MINE \\
\hline USCA & 48 & YUBA COUNTY DEPOSITS \\
\hline
\end{tabular}

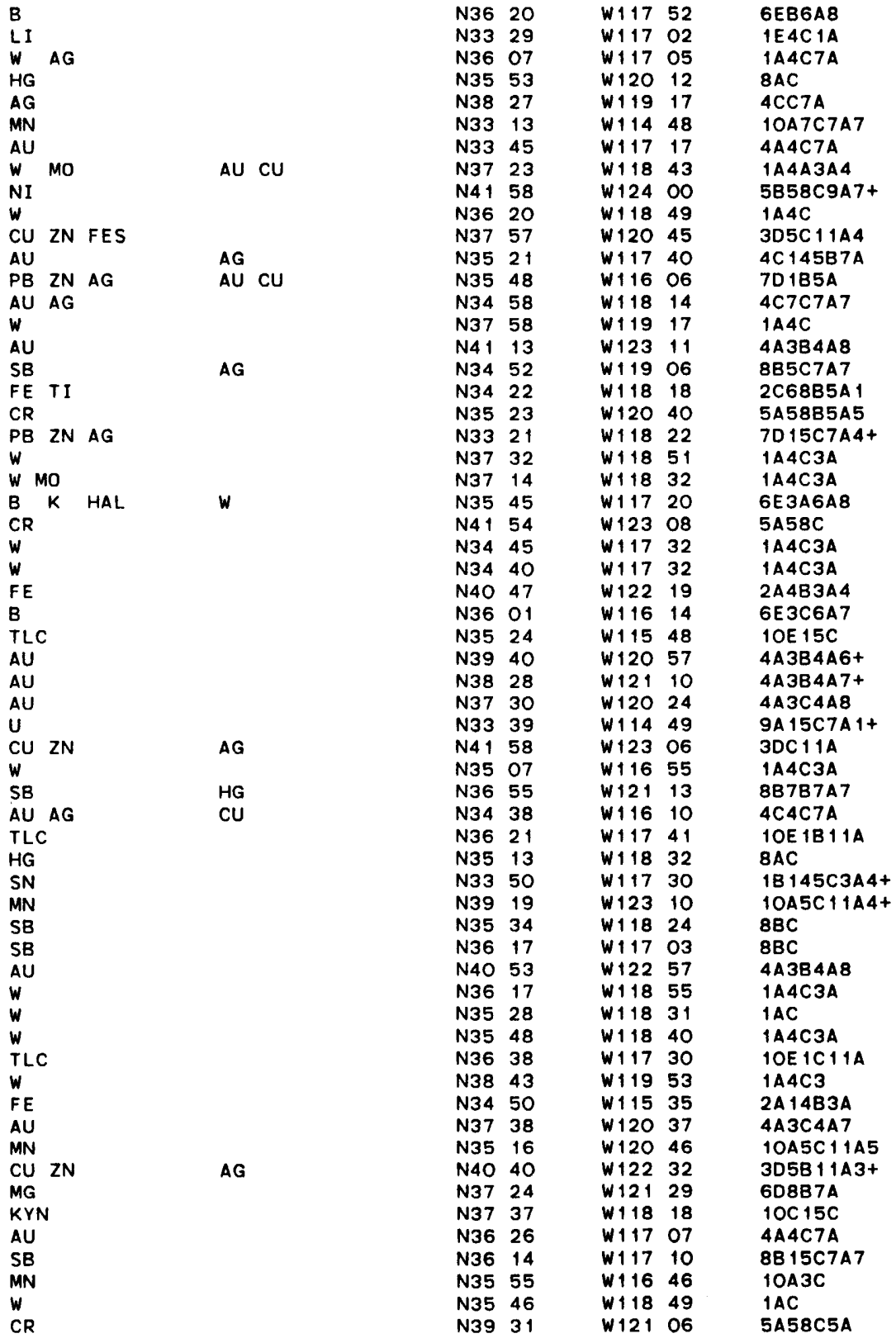


ALICE DISTRICT

ALMA DISTRICT (MOSQUITO, BUCKSKIN ET AL)

ASPEN (ROARING FORK) DISTRICT

AVERY RANCH DEPOSIT

BONANZA (KERBER CREEK) DISTRICT

BRECKENRIDGE DISTRICT

BROWNS CANYON-PONCHA SPRINGS DISTRICTS

BRUSH CREEK DISTRICT

CARIBOU HILL DEPOSIT

CASHIN MINE

CENTRAL CITY-IDAHO SPRINGS-TRAIL CREEK

CHALK CREEK DISTRICT (MARY MURPHY MINE)

CLIMAX MINE

COCHETOPA CREEK (LOS OCHOS) AREA

COPPER KING MINE (PRAIRIE DIVIDE)

CREEDE DISTRICT (AMETHYST, OH ET AL MINES) CRIPPLE CREEK DISTRICT

CRYSTAL (GREEN CRYSTAL) MINE

DORCHESTER-TAYLOR PARK DISTRICT

EMPIRE DISTRICT

EUREKA DISTRICT (SUNNYSIDE ET AL MINES)

EVERGREEN

GILMAN (RED CLIFF, BATTLE MTN) DISTRICT GOLD BRICK-PITKIN DISTRICT

GOLD HILL (SUGAR LOAF) DISTRICT

GOOD HOPE \& NEVADA GROUP

GRAND MESA DEPOSIT

GREEN MOUNTAIN DISTRICT (BIG FOUR MINE)

GRUNROCK, SPAULDING, LOOKOUT, CHALLENGER

GUFFEY DISTRICT

HAHNS PEAK

HOT SULFUR SPRINGS DEPOSITS

IRON HILL-CEBOLLA \& POWERHOUSE DISTRICTS

JAMESTOWN DISTRICT

JEFFERSON AREA

LA PLATA

LAKE CITY (GALENA LAKE) DISTRICT

LEADVILLE DISTRICT

MARSALL PASS AREA TUNNEL \& MASON RANCH)

MAYBELLLE

MCINTIRE, SANTA ROSA ET AL

AREA

MONARCH-TOMICHI-WHITE PINE DISTRICTS

MONTEZUMA (SNAKE RIVER) DISTRICT

MOUNT EMMONS (RED LADY BASIN) PROSPECT

NEDERLAND (BOULDER COUNTY) DISTRICT

NORTHGATE DISTRICT (CAMP CREEK ET AL MINES)

OLIVER PROSPECT

OPHIR (IRON SPRINGS) DISTRICT

PLACERVILLE (BEAR \& FALL CREEK) AREA

RALSTON CREEK (SCHWARTZWALDER MINE)

RICO (PIONEER) DISTRICT

RIFLE CREEK AREA

ROCK CREEK DISTRICT

ROSITA HILLS DIST (BASSICK ET AL MINES)

SILVERTON DISTRICT (SHENANDOAH ET AL MINES)

SNEFFELS-RED MTN-TELLURIDE DISTRICTS

SOUTH PLATTE DISTRICT

SUMMITVILLE DISTRICT
AU AG CU

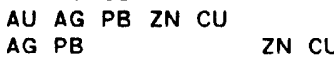

$A G \quad P B \quad Z N \quad C U$ AU

$A U$ AG PB ZN

$F$

AG

$A G C U$

$\triangle U A G$

AU AG $\quad$ CU PB ZN

$\begin{array}{ll}A U A G & P B C U Z N \text { MO } B E \\ M O & W \text { SN }\end{array}$

$u$

U

$A G$ PB ZN AU CU

$A U$

CU
CU PB ZN
PB CU ZN MO BE
$W \quad S N$

N39 18

N39 11

N38 31

N38 18

N39 29

N38 35

N39 35

N39 43

N38 19

N39 45

N38 40

N39 22

N38 21

N37 52

N38 43

N4O 4

N38 57

N39 44

PB AG

$A U$ AG PB ZN CU MN W F BA

AU

ZN AG CU PB AU MN

$\begin{array}{lll}Z N & A G & C U \\ A U & A G & P B\end{array}$

AU

$\begin{array}{ll}U & V \\ \text { TI } & \text { TH }\end{array}$

$\begin{array}{lll}\text { TI } & \text { TH } & \text { REE } \\ \text { ZN } & \text { AG }\end{array}$

AG PB

PB

$W$

AU

TH REE NB FE TI

$F$ AU AG PB ZN

AU

PB CU

$A G$ PB AU CU ZN

$A G$ ZN PB AU CU MN FE BI W

$u$

W AU

U V

$Z N \quad A G P B \quad C U A U$

$A G \quad P B \quad Z N$

$A U$ CU BI

MO

W

$A G \quad A U P B \quad C U Z N$

U

AG ZN PB AU CU

$A G P B$

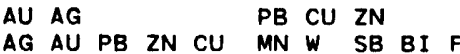

$A U$ AG PB CU ZN

AG CU PB
W105 39

W106 06

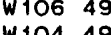

$\begin{array}{lll}W & 104 & 49 \\ W & 106 & -108\end{array}$

W106 08

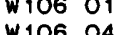

W106 04

$\begin{array}{ll}W 106 & 44 \\ W & 105 \\ W\end{array}$

W 10857

W105 32

$\begin{array}{lll}W & 21 \\ W & 106 & 1\end{array}$

W106 10

W106 44

W105 29

W106 54

W105 09

$W 10635$

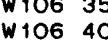

$\begin{array}{ll}W \\ W \\ w 105 & 40 \\ W & 40\end{array}$

$W 10544$
$W 10736$

$W 10736$
$W 105$
$W$

$W 10518$
$W 106$

$W 10623$
$W 10635$

W106 35

W105 23

W108 00

$W 108 \quad 19$

$W 10620$

$W 10524$

$W 10532$
$w 10657$

W106 57

W106 08

W107 04

W105 23

W105 48

W108 04

W107 22

$W 10722$
$W 106$
$W$

$\begin{array}{ll}W & 106 \\ w 106 & 22\end{array}$

W106 22

$\begin{array}{ll}W 105 & 10 \\ W & \end{array}$

W105 16*

$\begin{array}{ll}W 105 & 16 \\ W & 107 \\ W\end{array}$

$W 10743$
$W 10618$

$W 106 \quad 18$
$W 10552$

$W 10552$
$W 107$

$\begin{array}{ll}W 107 & 04 \\ W 105 & 27\end{array}$

W106 16

W105 30

$W 10750$

$W 10801$

W105 18

W108 01

W107 41

$W 107$
$W$

$W 10706$
$W 10520$

$W 10520$

W10736

$W 10745$
$W 105$
$W$

$W 10513$
$W 10636$
4C4C5A6+

4C4B7A6+

4D347B5A6+

9A37C 10A6+

4D47C7A7

404B7A6+

8C1457B7A7

2B 15C 1OA 1

4D37C 1OA5+

4C145B7A6

4D4C7A6

B4A5A7

9A37B7A7

9A15C7A7

4D7B7A7

4A27A5A7

$4 D C$

$4 C 4 C 5 A 6+$

4C4C5A6+

4D $47 B 7 A 7$
$8 C C 7 A$

7D347B5A6

7D347B5A6+

4D $145 C 7 A 6+$

9CC10A5+

2B37C4A6

704C7A6+

1A 15C 10A1

1A 15C 1OA 1

4A34C4AB

A37C 10A6+

BC 145B7A6

BC 145C7A

4C347C7A6+

4D7C7A7

4D347A5A6+

9A37B7A7

AA 15C 1OA

9A37B $10 B$

9AC

9C37C $10 A$

7D347C5A6+

4D 145C7A6

3B 4B5A7

1A 145A7A7

BC 15B7A7

$1 A 15 C$

4C7C7A7

9C37B 10A5+

9A $1587 A 7$

4D347B5A 7

9B37B 10A5+

4C47C7A7

4D47B7A7

11CC1A2

4A7C7A7 
$A G \quad A U P B \quad Z N$ $A U$ PB CU ZN BA SB RAP DISTRICT WEST CLIFF-SILVER CLIFF (HARDSCRABBLE) WET MOUNTAINS DISTRICT $U$

AMELIA ISLAND PROSPECT

BOULOGNE MINE

BRONSON (EAST OCALA HARD ROCK) DISTRICT

GREEN COVE SPRINGS MINE

INTERLACHEN PROSPECT

JACKSONVILLE DISTRICT

MELBOURNE-PALM BAY MINE

PENSACOLA BAY PROSPECT

PHILIPS INLET-PANAMA CITY PROSPECT

SOUTH GEORGIA-NORTH FLORIDA DISTRICT

SOUTH-CENTRAL (MAIN) DISTRICT

STEINHATCHEE (N. OCALA HARD ROCK) DISTRICT

TRATL RIDGE DISTRICT

VERO BEACH MINE

VULEE PROSPECT

ALTAMAHA PLANTATION AREA

ANDERSONVILLE DISTRICT

BLUE RIDGE AREA OF MURPHY MARBLE BELT

CARTERSVILLE BARITE DISTRICT

CARTERSVILLE MANGANESE DISTRICT

CHATSWORTH DISTRICT

CHESTATEE MINE

CREIGHTON (FRANKLIN) MINE

DAHLONEGA DISTRICT

DILLARDS AREA

ETON-RURALVALE DISTRICT

FOLKSTON AREA

HARRIS PROSPECT

JEKYLL ISLAND PROSPECT

MOUNTAIN CREEK AREA

NORTH FLORIDA PHOSPHATE DISTRIC

NORTHWEST GEORGIA BAUXITE DISTRICT

NORTHWEST GEORGIA BROWN ORE DISTRICT

NORTHWEST GEORGIA RED ORE DISTRICT

SAVANNAH BEACH PLACER PROSPECT

SAVANNAH DISTRICT

SEMINOLE (MAGRUDER) MINE

SOUTHWEST GEORGIA BROWN DRE DISTRICT

SPRINGVALE DISTRICT

TALLAPOOSA (TUDRO, WALDROP) MINE

TURNER PROSPECT

VILLA RICA

WALESKA AREA

ALBIA AREA

BUSSEY AREA

CENTERVILLE AREA
N38 34

N38 43

N38 04

N39 45

N38 27

N.37 43

N38 09

N38 13

N41 43

N41 29

N41 23

N3O 37

ZR KYN TH REE

N30 46

N29 20

N29 52

N29 52

N29 40

N30 18

N28 04

N3O 24

N3O 16

N29 45

N27 40

N29 54

N3O 03

N27 38

N3O 37

N31 17

N32 12

N34 50

N34 10

N34 15

N34 45

N34 33

N34 20

N34 20

N34 30

N34 57

N34 48

N3O 51

N33 59

N31 03

N32 48

N34 41

N3O 45

N34 17

N34 10

N34 52

N31 59

N32 03

N33 46

N32 00

N31 49

N33 51

N33 03

$\begin{array}{ll}\text { N33 } & 03 \\ N 33 & 47\end{array}$

N33 47

N34 17

N4 1 O3

N41 12

$\begin{array}{ll}\text { N4O } & 42 \\ \text { N42 } & 31\end{array}$
W105 27

W106 29

W107 40

W105 50

W108 44*

W106 47

W105 27

W105 18

W072 55

W072 54

W073 16

W081 27

W082 00

WO82 30*

WO8 142

WO8 153

WO8 133

W080 37

108701

08600

WO82 08*

W081 53*

WO83 19

WO82 02

WO8O 23

WOB 137

W081 29

W084 08

W084 21

W084 47

W084 45*

W084 42

W083 50

WO83 50

$\begin{array}{ll}\text { WO84 } & 17 \\ \text { WO8 } 400\end{array}$

W084 0

W083 26

WO8 48

WOB 58

WO84 52

W081 24

WO84 59

W083 42

WO83 00 *

W084 57*

W085 15*

WO85 19*

WO8O 53

WOB 1 05*

W082 34

WOB4 50*

WO84 52

W085 05

W085 05

W084 56

W084 54

W084 34
W092 48

W092 48

W093 01

W092 52
W094 07
9A37B $10 A 7$

1C 145B7A2

4D347C5A6+

3B4A5A7

9C37A 10B5+

8C7B7A7

4D 1457C7A6+

9D $125 C 7 A 2$

3A3C $10 A 7$

8D3C7A7+

1A 15C3A

2B37B4A8

2B37B4A8

2E37B2C7

2B37B4A8

B37C4A8

2B37B4A8

2B

2BJTC4AB

2B37C4A8

2E $37 A 2 B 7$

2E $37 B 2 C 7$

2B37A4A7-8

2B37B4A8

2B37C4A8

2B37C4A8

1OB37C9A7

IOE 1C 11A4+

BD 1B 1OA3

IOE 15B 11A3-4

3D 15C $11 A 2$

4A 15C7A

4A 15C7

4OEC

8D 1B 1OA4+

2B37B4A7-8

B $3704 A$

1OE 158C

4A 15C7A

2E37B2C7

1OB 1C9B8

$2 \mathrm{~A} 1 \mathrm{C} 9 \mathrm{C} 8$

2A 1C2B 4

2B37C4A8

2E37B2B 7

3E 15C7A

2A37C9C7+

3A 15C11A2-3

5A 158C5A

$11 B 15 C 11 A 2$

8D 1C10A2+

6A37C

6A37C

GA37B6A4 


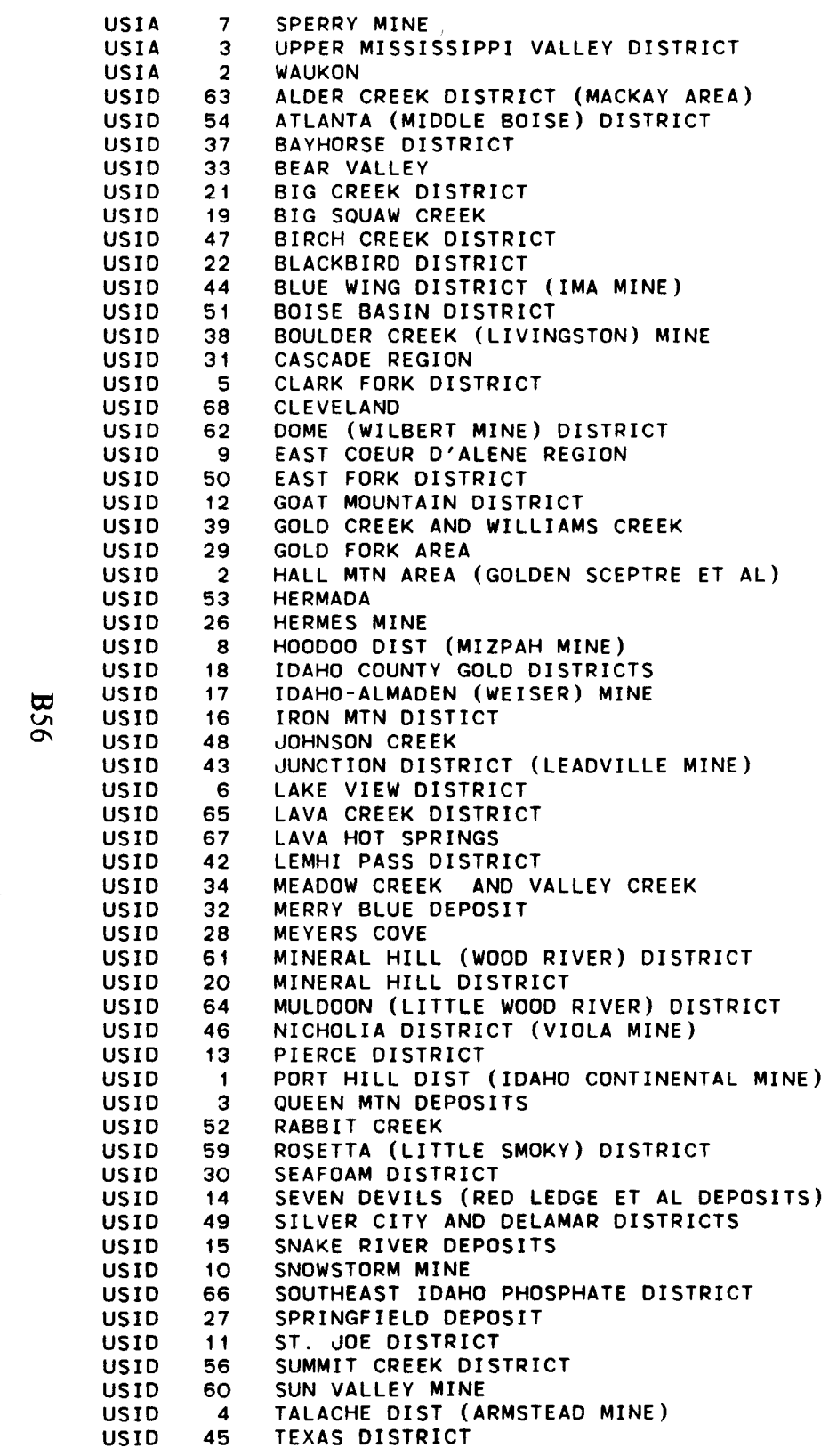

\begin{tabular}{|c|c|c|c|c|c|c|c|c|c|}
\hline GYP & & & & & & $\mathrm{N} 4 \mathrm{O}$ & 54 & Wo9 1 & 07 \\
\hline$Z N$ & PB & & & & & N42 & 31 & wo9o & $36 *$ \\
\hline $\mathrm{FE}$ & & & & & & N43 & 22 & WO9 1 & 30 \\
\hline $\mathrm{cu}$ & $\mathbf{W}$ & & $A G$ & PB & $\mathrm{ZN} \mathrm{FE}$ & N44 & 50 & $W 113$ & 44 \\
\hline$A U$ & $\Delta G$ & & & & & N43 & 47 & $W 115$ & 06 \\
\hline$A G$ & PB & $2 \mathrm{~N}$ & & & & N44 & 21 & W114 & 23 \\
\hline TI & & $U$ REE & & & & N44 & 19 & W115 & 23 \\
\hline$w$ & & & & & & N45 & 08 & $W+15$ & 22 \\
\hline $\mathrm{F}$ & & & & & & N45 & 29 & W114 & 58 \\
\hline PB & $\mathrm{ZN}$ & $A G$ & & & & N44 & 09 & $W 112$ & 49 \\
\hline co & $\mathrm{cu}$ & & NI & $A U$ & & N45 & 07 & $W 114$ & 20 \\
\hline w & & & $\mathbf{A G}$ & $P B$ & $\mathrm{CU}$ & N44 & 32 & $W 113$ & 42 \\
\hline$A U$ & & & $T I$ & & & N43 & 53 & W115 & 57 \\
\hline AG & PB & $Z N$ & $A U$ & $\mathrm{CU}$ & & N44 & 08 & $W 114$ & 36 \\
\hline TI & TH & REE NB & & & & N44 & 28 & $W 115$ & 57 \\
\hline PB & $A G$ & & $2 \mathrm{~N}$ & & & N48 & 10 & W116 & 10 \\
\hline$M N$ & & & & & & N42 & 18 & W111 & 42 \\
\hline PB & AG & & ZN & & & N43 & 58 & $W 113$ & oo \\
\hline PB & $A G$ & $Z \mathrm{~N}$ & $\mathrm{CU}$ & $A U$ & SB & N47 & 29 & W115 & 50 \\
\hline$A G$ & PB & $Z N$ & $A U$ & $\mathrm{CU}$ & & N43 & 59 & W114 & 39 \\
\hline KYN & & & & & & N46 & 56 & W115 & 52 \\
\hline TI & & & & & & N44 & 02 & $W 114$ & 49 \\
\hline TI & REE & & & & & N44 & 42 & $W 115$ & 59 \\
\hline $\mathrm{TH}$ & REE & & & & & N48 & 58 & W116 & 26 \\
\hline SB & & & & & & N43 & 52 & W115 & 22 \\
\hline$H G$ & & & & & & N44 & 55 & W115 & 12 \\
\hline CU & & & & & & N47 & $\infty 0$ & $W 116$ & 30 \\
\hline$A U$ & & & $\mathrm{TH}$ & TI & REE & N45 & 35 & W115 & $40 *$ \\
\hline HG & & & & & & N44 & 13 & W116 & 39 \\
\hline FE & FES & & & & & N44 & 30 & $W 116$ & 58 \\
\hline TI & & & & & & N43 & 54 & $W 116$ & 18 \\
\hline$A G$ & PB & $\mathrm{ZN}$ & $A U$ & $\mathrm{CU}$ & & N44 & 42 & W113 & 18 \\
\hline$A G$ & PB & & $\mathrm{ZN}$ & $\mathrm{CU}$ & & N47 & 54 & $w+16$ & 27 \\
\hline$A G$ & PB & $\mathrm{ZN}$ & & & & N43 & 33 & $W 113$ & 37 \\
\hline$M N$ & & & & & & N42 & 34 & W111 & 59 \\
\hline $\mathrm{TH}$ & REE & & & & & N44 & 55 & $W 113$ & 29 \\
\hline T I & & & TH & $2 R$ & & N44 & 18 & W115 & 01 \\
\hline$w$ & & & & & & N44 & 21 & W115 & 35 \\
\hline $\mathrm{F}$ & & & $B A$ & & & N44 & 50 & $w+14$ & 30 \\
\hline$A G$ & PB & $Z N$ & SB & & & N43 & 29 & $W 114$ & 22 \\
\hline TH & REE & NB & & & & N45 & 24 & W114 & 11 \\
\hline AG & PB & $\mathrm{ZN}$ & & & & N43 & 37 & $W 113$ & 53 \\
\hline PB & $Z N$ & $A G$ & & & & N44 & 22 & $W 112$ & 59 \\
\hline$A U$ & & & & & & N46 & 30 & W115 & 47 \\
\hline$A G$ & PB & & $\mathrm{ZN}$ & $\mathrm{CU}$ & & N48 & 56 & $W 116$ & 54 \\
\hline$W$ & & & & & & N48 & 54 & $W+16$ & 17 \\
\hline TI I & & & & & & N43 & 48 & $w+15$ & 41 \\
\hline$A G$ & PB & $Z N$ & $\mathbf{A U}$ & $\mathrm{CU}$ & & N43 & 36 & $W 114$ & 42 \\
\hline$A G$ & PB & $\mathrm{ZN}$ & $A U$ & $\mathrm{CU}$ & & N44 & 35 & $W 115$ & 04 \\
\hline $\mathrm{CU}$ & & & $\mathrm{AU}$ & $\mathbf{A G}$ & ZN & N45 & 14 & $w 116$ & 40 \\
\hline AG & $A U$ & SB & & & & N43 & 01 & Wi16 & 46 \\
\hline GYP & & & & & & N44 & 29 & $w 117$ & 13 \\
\hline $\mathrm{cu}$ & & & $A G$ & & & N47 & 28 & $w+15$ & 44 \\
\hline P & & & & & & N42 & 53 & $W 111$ & $45 *$ \\
\hline$w$ & & & & & & N44 & 47 & $W 115$ & 23 \\
\hline $\mathrm{cu}$ & & & & & & N47 & 12 & W115 & 30 \\
\hline$w$ & & & & & & N43 & 52 & $W 114$ & 12 \\
\hline BA & & & & & & N43 & 33 & $W 114$ & 28 \\
\hline$A G$ & PB & & $A U$ & $\mathrm{CU}$ & & N48 & 08 & $W 116$ & 29 \\
\hline PB & $\mathrm{ZN}$ & AG & & & & N44 & 28 & $W 113$ & 18 \\
\hline
\end{tabular}

6A37C6A4 7C37A 10B3 2A37C9A7 4A 4C7A 4D14B5A5+ 2B3B4A8 $1 A C$

8C 145C7A 7014C7A 5C 15A 11A 1A14587A 4A3B4A8 4D 14C5A 2B3B 4 A8 $7014 C 7 A$ 1OA3C $11 A 8$ $7 D+4 C 5 A$ 7D TA7A 4D 14C7A 10C 15B 2B3C4A8 2B3C $4 A 8$ 9D 14B7A $8 B C$

8A 14B7A

3A 1C 11A

AA $3 B 4 B 7-8$
$8 A 3 B 10 A 7$

2DC5A

2B3C4A8

4D 14C5A

4D 14C7A

4D47C7A

1OA3C $11 A 8$

9D 1A7A

$2 B 3 C 4 A 8$

1AC7A

8C47C7A7

4D 14B7A

$9 D 15 C$

$4014 C 5 A$

7D 14C

4A3C4AB

4D 14C7A

1AC7A

2B3C4A8

4D 14C5A

4014C5A

3A5A 1 1A4

4C7B7A7

6A37C6A 4

3A 1C 1OA

2E37A2B3

IAC

$3 C 1 C$

1A4C3A

8DC11A

4D 14C7A 
YELLOW PINE, MEADOW CREEK. ANTIMONY RIDGE

ILLINOIS-KENTUCKY FLUORSPAR DISTRICT UPPER MISSISSIPPI VALLEY DISTRICT SHOALS

BLUE RAPIDS AREA

DILLON AREA

ELLSWORTH AREA
HUTCHINSON AREA

LYONS AREA

SUN CITY MINE

TRI - STATE DISTRIC

BURKESVILLE AREA

CENTRAL KENTUCKY BROWN ROCK DISTRICT CENTRAL KENTUCKY FLUORITE DISTRICT

GRATZ VEIN AREA

PARIS-MILLERSBURG AREA

WESTERN KENTUCKY BROWN IRON DISTRICT

WESTERN KENTUCKY FLUORSPAR DISTRICT

CHACAHOULA MINE

GARDEN ISLAND BAY

GRAND ISLE (BLK. 18) MINE

JEFFERSON ISLAND MINE

LAFAYETTE AREA

LAKE WASHINGTON (GRANO ECAILLE) MINE

LAKE WASHINGTON (GRANO ECAILL

PLAQUEMINE AREA

STARKS DOME

SULPHUR MINE

WEEKS ISLAND-GRANDE COTE

WINNF IELD MINE

CHESTERFIELD DISTRICT

DAVIS MINE

GREAT FALLS (FORD, WATSON, MARYLAND MINES)

LINGANORE DIST (NEW LONDON \& LIBERTY MINES) MOUNT WASHINGTON (BARE HILLS) MINE

REED (WILKINS) MINE

SOLDIERS DELIGHT

SYKESVILLE DISTRICT

BALD MOUNTAIN (MACHIAS LAKE)

BARRETT-BIG HILL PROSPECT

BLACK HAWK-BLUE HILL DISTRICT

CENTRAL AROOSTOOK COUNTY DISTRICT

CRAWFORD PONO DEPOSITS

KATAHDIN PYRRHOTITE

LEDGE RIDGE (PARMACHENEE TOWNSHIP)

MOUNT MICA AREA

NORTHERN AROOSTOOK COUNTY DISTRICT

PENOBSCOT (HARBORSIDE) DISTRICT

SOUTHERN AROOSTOOK COUNTY DISTRICT

$Z N$ PB $A G$ AU CU

PB AG ZN CU AU SB

U

$A G \quad A U$

W AU SB

$S B$

ZN PB

ZN PYP

GYP

GYP

HAL

HAL

HAL

ZN PB

$B A$ ZN

$P$

F $B A$

BA ZN

FE

FE

S

S

HAL

$S$

SE

HAL

$\mathrm{S}$

HAL

GYP

$\begin{array}{ll}\text { FES } & \text { CU } \\ A U & \\ C U & \text { ZN AG }\end{array}$

CU

CR

FE ZN CO

ZN CU

$Z N$ CU

ZN CU FES

MN FE

CU PB ZN

PB AG AU

PB AG

MN FE

ZN CU FES

CU

SN

MN FE
N44 19

N43 48

N43 40

N47 31

N44 03

N43 47

N45 04

N44 21

N44 55

N44 55

N44 55

N47

N42 31

N39 42

N38 39

N38 4

N38 02

N38 23

N37 18

N37 00

N36 51

N38 06

N37 54

N38 25

N38 16

N38 08

N36 50

N37 19

N29 48

N29 12

N29 12

N3O 15

N29 06

N29 21

N32 45

N3O 17

N3O 1

N3O 18

N29 5

N31 55

N42 25

N42 41

N39 06

N39 29

N39 23

N39 37

N39 25

N39 24

N46 44

N44 58

N44 23

N46 22

N44 15

N45 26

N45 15

N44 16

N46 47

N44 21

N46 04
11432

W114 48

$\begin{array}{lll}W 114 & 17\end{array}$

W116 09

W114 34

$\begin{array}{ll}W 114 & 06 \\ W & 113 \\ W 2\end{array}$

W114 43

W115 20

$W 11520$
$W 11527$

$\begin{array}{ll}W 115 & 27 \\ W 088 & 17\end{array}$

W088 17

W086 41

W096 39

W097 12

W098 14

W097 57

W098 12

W098 56

WO94 30*

WO85 17

W084 43*

WOB 455

WO84 10

W083 36

W088 10*

W088 11

W088 11

W090 58

W089 09

W091 58

W091 58

W091 55

w090 36

WO93 30*

WO9 17

W093 37

W093 22

wo9 147

W092 42

WO72 48*

W072 52

W077 16

W077 15

W076 40

W076 26

W076 50

W076 57

W068 47

W067 13

W068 39

W068 00

W069 19

W069 12

W071 03

W070 $27 *$

W068 10

W068 46

W067 55
3B 14B5A5

4D4C7A

3B 14B5A6

1A4B3A

9A7C7A7

IA4B7A

1A4B7A

8C2378A5A5-7

7C37A 10B3+

6A37C6A4

GA37B6A4

6A37B6A4

6B37B6X4

$6 B 37 B 6 \times 4$

$6 B 37 B 6 \times 4$

6B37B6A4

7C37A 10B4+

BD37C 1OA

E37C9B8

C37B7B3+

D37C10A3+

$2 A 37 C 2 A 3$

2A37C9C4t

8C2378A5A4-6

11 A3B5A5+

1A3A5A5+

1 1 A3A5A5+

$11 A 3 B 5 A 5+$

$6 B 3 B 6 \times 5$

11A3B5A5+

11A3A5A5T

A

政

11 A3B5A5+

3B6 55

6A3C6A5

B4C $1 B$

3015C11A

उA 1C 1 1 A -3

5A8B5A3-

-

3A6C 1 1A2-3

3D5A 11A3

3D5C $10 A 4$

3D5B $11 A 4$

1OA5A2A4

5B58C5A

$11 B 6 C 5 A 4$

$3 D 5 C 11 A 3$

1BC $1 B$

305B11A4

10A5B2A4 


\begin{tabular}{|c|c|c|}
\hline USME & 12 & TUNK POND \\
\hline USMI & 16 & ALABASTER MINE \\
\hline USMI & 5 & AMERICAN METALS PROSPECT \\
\hline USMI & 14 & BEAVER I SLAND \\
\hline USMI & 8 & BUSCHELL LAKE \\
\hline USMI & 19 & DETROIT SALT \\
\hline USMI & 2 & EAST GOGEBIC RANGE \\
\hline USMI & 11 & FELCH TROUGH (GROVELAND MINE) \\
\hline USMI & 18 & GRAND RAPIDS ET AL MINES \\
\hline USMI & 10 & GWINN \\
\hline USMI & 6 & IRON RIVER-CRYSTAL FALLS DISTRICT \\
\hline USMI & 1 & ISLE ROYALE \\
\hline USMI & 3 & KEWEENAW COPPER DISTRICT \\
\hline USMI & 7 & MARQUETTE RANGE \\
\hline USMI & 12 & MENOMINEE RANGE \\
\hline USMI & 17 & MIDLAND \\
\hline USMI & 15 & NATIONAL CITY ET AL MINES \\
\hline USMI & 9 & REPUBLIC TROUGH \\
\hline USMI & 13 & ST. IGNACE DEPOSITS \\
\hline USMI & 4 & WHITE PINE MINE \\
\hline USMN & 2 & CUYUNA RANGE \\
\hline USMN & 1 & DULUTH GABBRO BELT \\
\hline USMN & 7 & FILLMORE COUNTY \\
\hline USMN & 6 & LAKE COUNTY (INCO) \\
\hline USMN & 3 & MESABI RANGE \\
\hline USMN & 5 & MINNAMAX \\
\hline USMN & 4 & VERMILION RANGE \\
\hline USMO & 24 & ANNAPOLIS DISTRICT \\
\hline USMO & 10 & AURORA DISTRICT \\
\hline USMO & 22 & BOSS MINE \\
\hline USMO & 12 & CAULFIELD DISTRICT \\
\hline USMO & 1 & CENTRAL DISTRICT \\
\hline USMO & 8 & CORRY MINES AREA \\
\hline USMO & 25 & EMINENCE DISTRICT \\
\hline USMO & 21 & FREDERICKTOWN DISTRICT \\
\hline USMO & 5 & GRANBY DISTRICT \\
\hline USMO & 18 & IRON MOUNTAIN \\
\hline USMO & 2 & OSAGE RIVER DISTRICT \\
\hline USMO & 11 & OZARK DISTRICT \\
\hline USMO & 3 & PEA RIDGE-BOURBON DISTRICT \\
\hline USMO & 9 & PIERSON CREEK (SPRINGF IELD) DISTRICT \\
\hline USMO & 19 & PILOT KNOB \\
\hline USMO & 14 & POTOSI DISTRICT \\
\hline USMO & 16 & SAINTE GENEVIEVE AREA \\
\hline USMO & 20 & SILVER MINE \\
\hline USMO & 13 & SOUTHEAST MISSOURI BROWN ORE DISTRICTS \\
\hline USMO & 17 & SOUTHEAST MISSOURI LEAD BELT \\
\hline USMO & 7 & SPRINGF IELO BROWN ORE DISTRICT \\
\hline USMO & 15 & STEELVILLE (CHERRY VALLEY) DISTRICT \\
\hline USMO & 4 & TRI-STATE DISTRICT \\
\hline USMO & 23 & VIBURNUM TREND (NEW LEAD BELT) \\
\hline USMO & 6 & WENTWORTH DISTRICT \\
\hline USMS & 3 & CAT ISLAND \\
\hline USMS & 2 & NORTH CENTRAL MISSISSIPPI DISTRICT \\
\hline USMS & 1 & NORTH MISSISSIPPI DISTRICT \\
\hline USMS & 4 & SHIP ISLAND \\
\hline USMT & 19 & AREA NE OF CHOTEAU \\
\hline USMT & 70 & ARGENTA DISTRICT \\
\hline USMT & 26 & ARGO, MINE \\
\hline USMT & $10 A$ & BABBIT MINE \\
\hline
\end{tabular}

\begin{tabular}{|c|c|c|c|c|}
\hline$w$ & & & N44 & 45 \\
\hline GYP & & & N44 & 12 \\
\hline $\mathrm{CU}$ & & $A G$ & N46 & 41 \\
\hline GYP & & & N45 & 44 \\
\hline CU & & & N46 & 30 \\
\hline HAL & & & N42 & 23 \\
\hline FE & & & N46 & 28 \\
\hline FE & & & N45 & 59 \\
\hline GYP & & & N42 & 57 \\
\hline FE & & & N46 & 16 \\
\hline $\mathrm{FE}$ & & & N46 & 04 \\
\hline $\mathrm{CU}$ & & & N48 & 04 \\
\hline $\mathrm{CU}$ & & $A G$ & N47 & 14 \\
\hline$F E$ & & & N46 & 30 \\
\hline FE & & & N45 & 50 \\
\hline HAL & & $k$ & N43 & 24 \\
\hline GYP & & & N44 & 16 \\
\hline FE & & & N46 & 26 \\
\hline GYP & & & N44 & 53 \\
\hline $\mathrm{CU}$ & & $A G$ & N46 & 46 \\
\hline FE & & MN & N46 & 30 \\
\hline FE & $T I$ & v & N48 & 02 \\
\hline FE & & & N43 & 37 \\
\hline $\mathrm{CU}$ & NI & PT & N47 & 51 \\
\hline FE & & & N4 7 & 30 \\
\hline $\mathrm{CU}$ & NI & PT & N47 & 40 \\
\hline FE & & & N47 & 48 \\
\hline $\mathrm{PB}$ & & $\mathrm{ZN} \mathrm{CU}$ & N37 & 19 \\
\hline $\mathrm{ZN}$ & PB & & N36 & 57 \\
\hline FE & $\mathrm{cu}$ & & N37 & 33 \\
\hline $\mathbf{Z N}$ & & & N36 & 35 \\
\hline BA & PB ZN & & N38 & 22 \\
\hline $2 N$ & PB & & N37 & 28 \\
\hline$C U$ & & & N37 & 06 \\
\hline PB & & $C U N I \quad C O Z N$ & N37 & 34 \\
\hline $2 N$ & PB & & N36 & 52 \\
\hline FE & & & N37 & 42 \\
\hline FE & & & N38 & 15 \\
\hline PB & $2 \mathrm{~N}$ & & N36 & 57 \\
\hline FE & & & N38 & 11 \\
\hline $\mathbf{Z N}$ & PB & & N37 & 10 \\
\hline FE & & & N37 & 35 \\
\hline BA & & PB ZN & N37 & 58 \\
\hline $\mathrm{CU}$ & & & N37 & 54 \\
\hline$w$ & & $A G P B S N$ & N37 & 30 \\
\hline FE & & & N36 & 30 \\
\hline PB & & $Z N$ CU AG & N37 & 49 \\
\hline FE & & & N37 & 30 \\
\hline FES & 5 FE & & N37 & 56 \\
\hline $2 \mathrm{~N}$ & PB & $C D$ & N36 & 56 \\
\hline PB & & ZN CU AG NI & N37 & 27 \\
\hline$Z N$ & & PB & N36 & 56 \\
\hline & & TI & N3O & 13 \\
\hline FE & & & N33 & 30 \\
\hline FE & & & N34 & 15 \\
\hline$T I$ & & & N3O & 13 \\
\hline FE & TI REE TH & & N47 & 53 \\
\hline $\mathbf{A G}$ & PB AU & $\mathrm{ZN} \mathrm{CU}$ & N45 & 18 \\
\hline w & $A U$ & & N46 & 35 \\
\hline SB & & & N47 & 35 \\
\hline
\end{tabular}




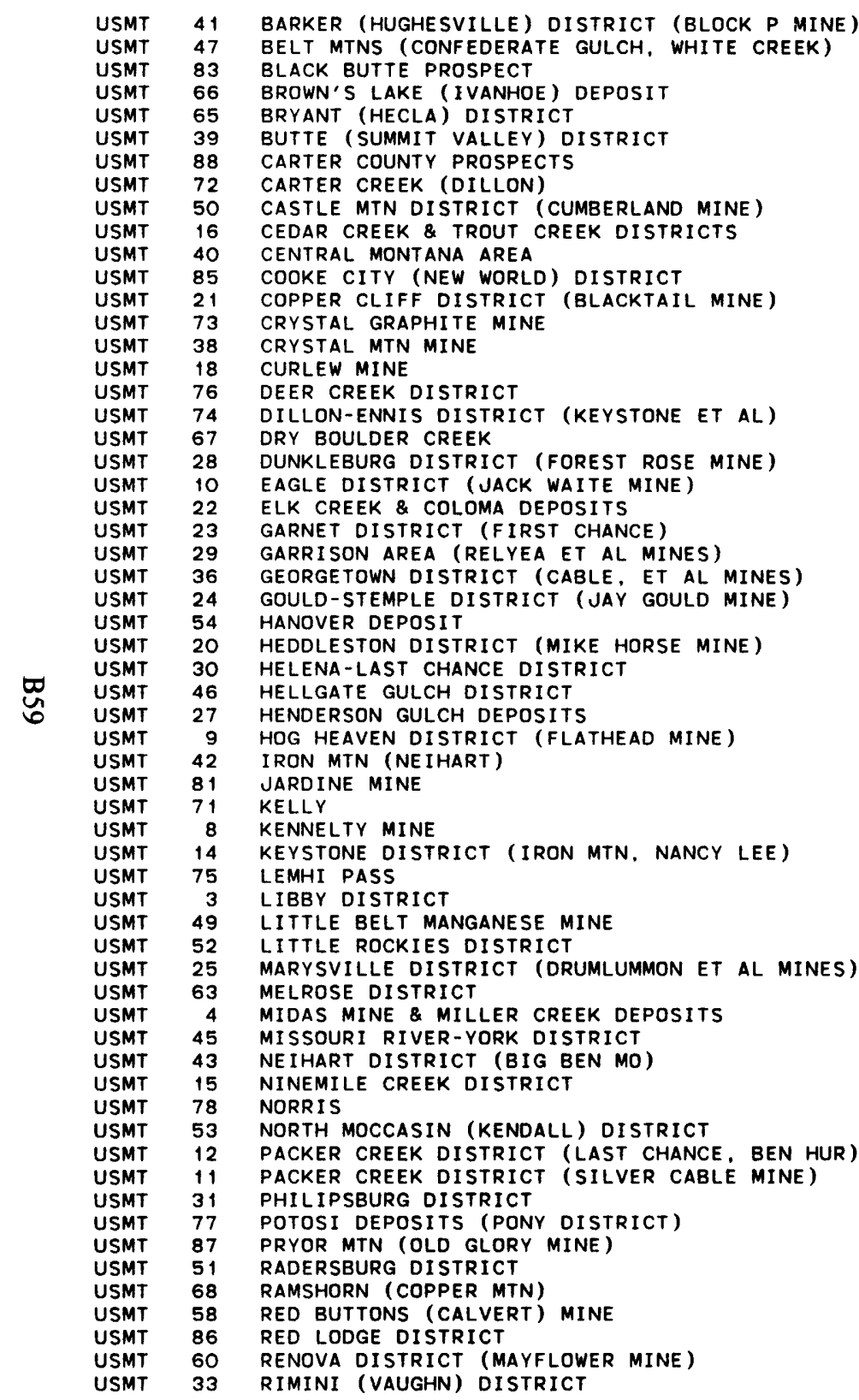

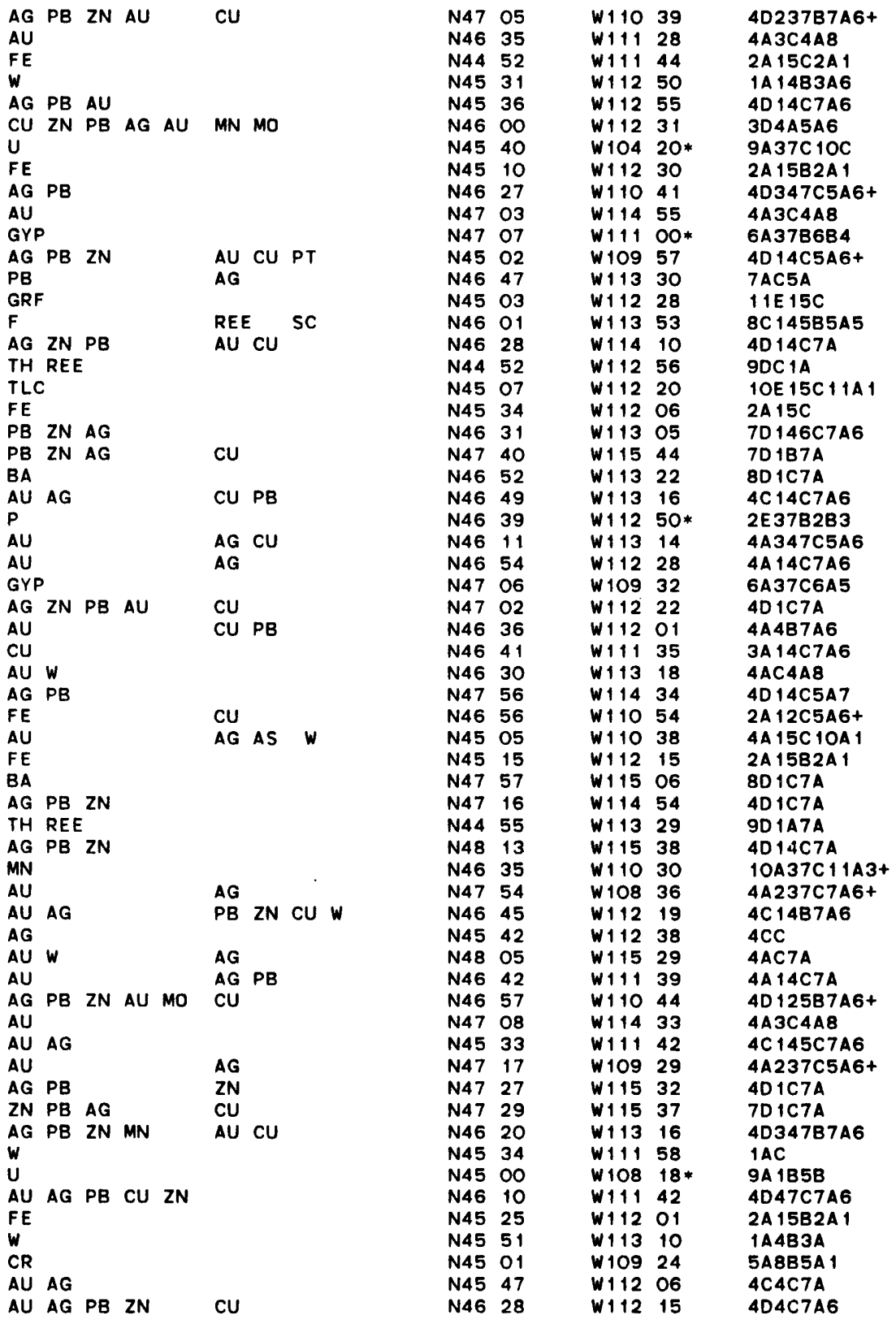




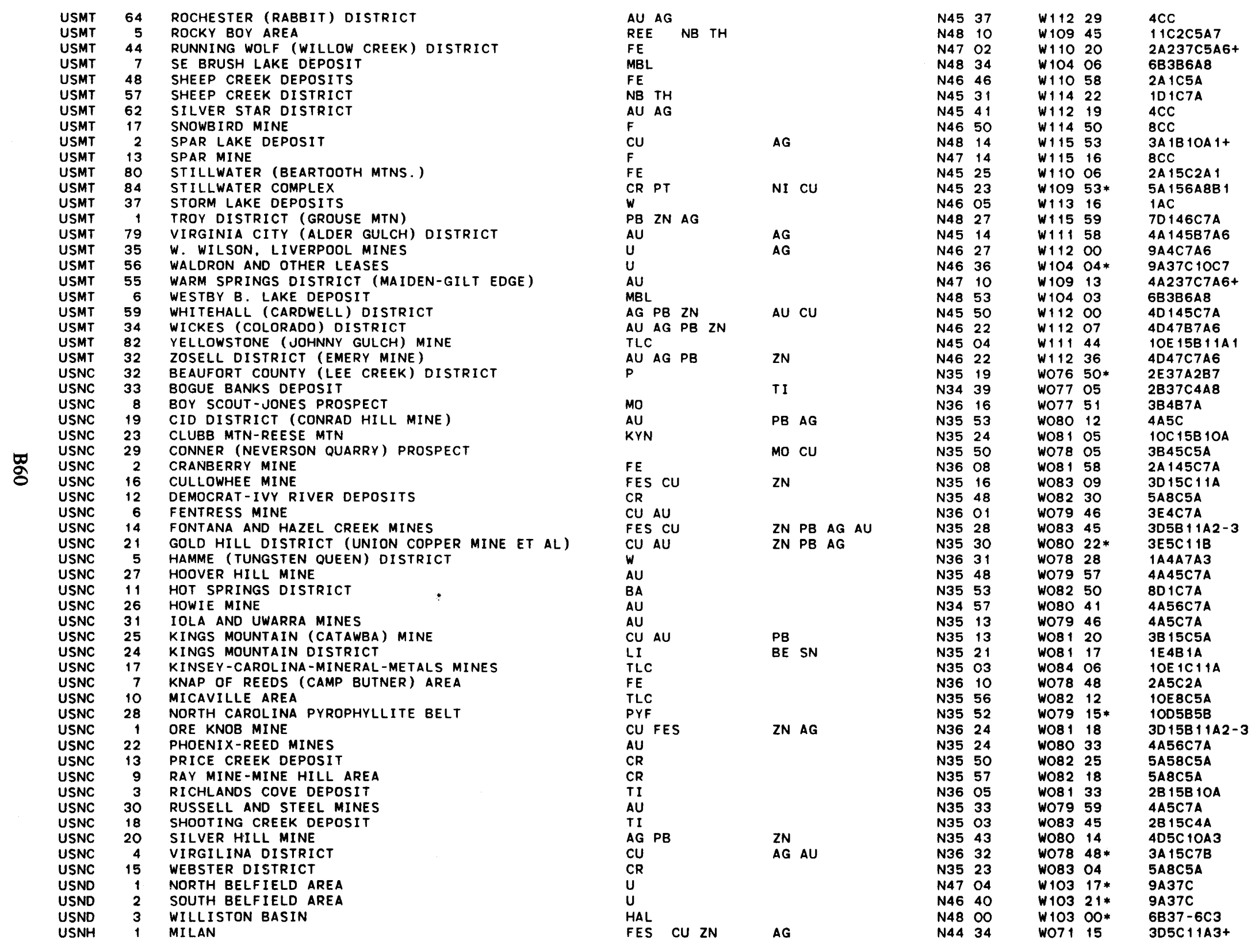


AMBROSIA LAKE DISTRICT

BISH CLAIMS

BOSTON HILL MINE, SILVER CITY AREA

BURRO MTN DIST (BURRO CHIEF \& SHRINE)

BURRO MTN DIST (TYRONE AREA)

CABALLO MTS AREA

CENTRAL (SANTA RITA) DIST (CHINO ET AL)

CERILLOS DISTRICT

CHARLEY 2 DEPOSIT

CHUSKA DISTRI

COCHITI DISTRICT (ALBEMARLE MINE)

COLLINS-GOODNER GROUP

COOKE'S PEAK DISTRIC

DELAWARE BASIN

EAST CARRIZO MTS (SHIPROCK) DISTRICT

EL RITO DEPOSIT

ELIZABETHTOWN-BALDY MTN DISTRICTS

FIERRO-HANOVER DISTRICT

FLUORITE RIDGE DISTRIC

GALLINAS MTS DISTRICT

GALLUP DISTRICT

GILA DISTRICT

GOOD LUCK CLAIMS

HACHITA (EUREKA) DISTRICT

HANSONBURG (CARTHAGE) DISTRICT

HARDING MINE

HEMBRILLO \& RED ROCK MINES

HERMOSA (PALOMAS) DISTRICT

HILLSBORO DISTRICT

HOOK RANCH (JARALOSA) AREA

HUCKLEBERRY DEPOSIT

JARILLA (OROGRANDE) DISTRICT

KINGSTON DISTRICT

LA BAJADA MINE

LA MADERA DEPOSIT

LA VENTANA MESA AREA

LAGUNA DISTRICT

LAKE VALLEY DISTRICT

LITTLE FLORIDA MTS DISTRICT

LORDSBURG DIST (85, MISERS CHEST, BONNEY)

LUCKY DON \& LITTLE DAVIE MINES

LUIS LOPEZ OISTRICT

MAGDALENA DISTRICT

MANZANO MTS DEPOSITS

MOGOLLON DISTRICT

NACIMIENTO MTS (CUBA) DISTRICT

NEW PLACERS DIST (ORTIZ, DOLORES MINES)

NORTHERN SIERRA CABALLO DISTRIC

ZN
CU
TI

1074

MN

U

\section{FE W BE}

CU

PB

CU U

N33 02

N31 54

N33 50

N36 11

N32 58

N33 10

N32 58

N34 18

N33 16

$\begin{array}{ll}\text { N33 } & 28 \\ \text { N32 } & 25\end{array}$

N32 55

N35 31

N36 27

AG PB MN MO $V$

MN F

$\mathrm{CU}$

U

ZN PB AG CU AU

AU AG PB ZN MO

N35 07

N32 44

N32 12

AG $A U$

PB BA

N32 18

N34 04

PB ZN AG AU CU

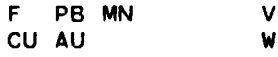

CU PB ZN

N34 58

N33 23

N36 00

N35 15

N33 06

N35 20

W074 35

1074 59

W074 20

W107 50*

W 10425

$\begin{array}{ll}W 108 & 17\end{array}$

W 10825

$\begin{array}{ll}W 108 & 23 \\ W 107 & 13\end{array}$

W103 57

W108 04

W106 09

W107 01

W107 50

W109 00

W106 33

$\begin{array}{ll}W 106 & 54 \\ W 107 & 43\end{array}$

W107 43

$\begin{array}{ll}W 103 & 30 * \\ W & 09 \\ W & 109\end{array}$

W106 10

W105 11

W108 04

$W 10743$
$W$

W105 45

W108 33

W108 30

$\begin{array}{ll}W 103 & 34 \\ W & \end{array}$

$W 10825$
$W 10621$

$\begin{array}{ll}W 106 & 21 \\ W 105 & 46\end{array}$

$\begin{array}{ll}W 105 & 46 \\ w 106 & 32\end{array}$

W107 43

$\begin{array}{ll}W 107 & 31 \\ W 107 & 25\end{array}$

$W 10725$

W108 49

W106 06

W107 43

$\begin{array}{ll}W 106 & 12\end{array}$

W105 58

W106 57

$\begin{array}{ll}W 107 & 19 \\ W & 107 \\ W 4\end{array}$

W 10734 *

W 10846

$\begin{array}{ll}W 108 & 46 \\ W 106 & 42\end{array}$

W106 58

W $107 \quad 12$

W106 23

W108 48

W106 53

$\begin{array}{lll}W 126 & 12\end{array}$

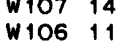

9A37C 10A5+

4C4C7AG+

9A37C10A5+

(B)37-6C4

9 C37C 10B5+

BCC

4A347C7A7

2D347B3A6

8C4C7A

$70 \mathrm{C}$

9A37B 10A5+

8C7B7A7

9A37C 1OA5+

4D4C7A6

7D347C7A6+

TE 145C1A2 -

IOE 16C $11 A 2$

4D37C5A

4A47C7A7

9A37C10A7

8 C7C7A7

3 A 347 C $3 A$

4D347C5A6

3A7C5A7

$8 \mathrm{CC}$

9A37C 1OA5+

9A37A 10A5

4D37C5A

10A3C7B7

3A4B7A6

9A37C5A4+

$7 D 347 B 3 A 7$

$8 \mathrm{CC}$

4C7B7A7

3A37C 10A5+

70347C5A

3A347C3A 7 


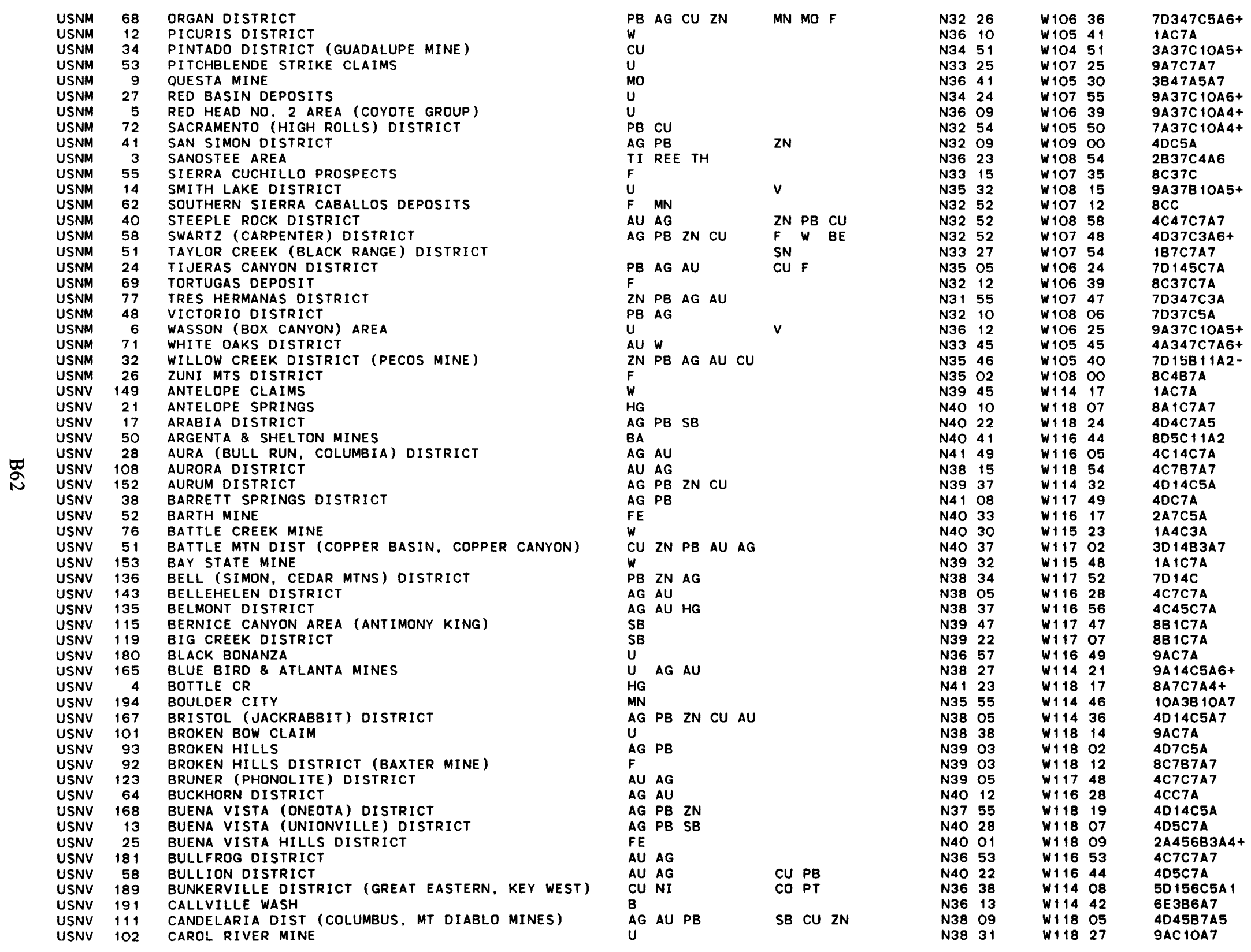




\begin{tabular}{|c|c|c|}
\hline $\begin{array}{l}\text { USNV } \\
\text { USNV }\end{array}$ & $\begin{array}{l}148 \\
147\end{array}$ & $\begin{array}{l}\text { CHERRY CREEK (EGAN CANYON) DISTRICT } \\
\text { CHERRY CREEK DISTRICT (TICUP MINE \& OTHERS) }\end{array}$ \\
\hline USNV & 146 & COLUMBUS MARSH \\
\hline USNV & 86 & COMSTOCK DISTRICT \\
\hline USNV & 73 & CONTACT (SALMON RIVER) DISTRICT \\
\hline USNV & 31 & CORNUCOPIA DISTRICT \\
\hline USNV & 67 & CORTEZ DISTRICT \\
\hline USNV & 68 & COTTONWOOD CANYON DISTRICT (LOVELOCK, NICKEL) \\
\hline USNV & 177 & CUCOMUNGO PROSPECT \\
\hline USNV & 162 & CURRANT CREEK DISTRICT \\
\hline USNV & 85 & DAYTON \\
\hline USNV & 1 & DEFENSE MINE \\
\hline USNV & 74 & DELANO DISTRICT (CLEVELAND \& DELANO MINES) \\
\hline USNV & 84 & DIXIE MINE \\
\hline USNV & 39 & DUTCH FLAT \\
\hline USNV & 128 & EAST NORTHUMBERLAND CANYON DEPOSIT \\
\hline USNV & 29 & EDGEMONT (CENTENNIAL) DISTRICT \\
\hline USNV & 196 & ELDORADO DISTRICT \\
\hline USNV & 156 & ELY (ROBINSON) OISTRICT \\
\hline USNV & 41 & ESTABROOK \& ROSSI MINES \\
\hline USNV & 154 & EUREKA DISTRICT \\
\hline USNV & 89 & FAIRVIEW DISTRICT \\
\hline USNV & 186 & FERGUSON (DELAMAR) DISTRICT \\
\hline USNV & 170 & FISH LAKE \\
\hline USNV & 169 & FISH LAKE VALLEY \\
\hline USNV & 182 & FLUORINE DISTRICT (DAISY MINE) \\
\hline USNV & 131 & GABBS (MAMMOTH) DISTRICT \\
\hline USNV & 98 & GARDNERVILLE (EAGLE) DISTRICT \\
\hline USNV & 72 & GARNET TUNGSTEN \\
\hline USNV & 9 & GERLACH \\
\hline USNV & 37 & GETCHELL MINE \\
\hline USNV & 45 & GOLCONDA \\
\hline USNV & 63 & GOLD ACRES MINE \\
\hline USNV & 192 & GOLD BUTTE AREA \\
\hline USNV & 35 & GOLD CIRCLE (MIDAS) DISTRICT \\
\hline USNV & 48 & GOLD RUN (ADELAIDE) DISTRICT \\
\hline USNV & 174 & GOLDFIELD DISTRICT \\
\hline USNV & 32 & GOOD HOPE DISTRICT \\
\hline USNV & 62 & GREYSTONE MINE \\
\hline USNV & 187 & GROOM MINE \\
\hline USNV & 140 & HALL PROSPECT (LIBERTY) \\
\hline USNV & 105 & HAWTHORNE DISTRICT \\
\hline USNV & 117 & HILLTOP MINE \\
\hline USNV & 90 & HOLY CROSS DISTRICT \\
\hline USNV & 178 & HORNSILVER DISTRICT \\
\hline USNV & 161 & HUB (SNAKE RANGE) MINE \\
\hline USNV & 11 & HUMBOLDT (IMLAY) DISTRICT \\
\hline USNV & 151 & HUNTER DISTRICT \\
\hline USNV & 114 & IOWA CANYON MINE \\
\hline USNV & 40 & I VANHOE \\
\hline USNV & 44 & IZENHOOD RANCH \\
\hline USNV & 122 & JACKSON (GOLD PARK) DISTRICT \\
\hline USNV & 3 & JACKSON MTNS DISTRICT \\
\hline USNV & 69 & JARBRIDGE DISTRICT \\
\hline USNV & 132 & JEFFERSON CANYON DISTRICT \\
\hline USNV & 33 & JERRITT (MARLBORO) CANYON \\
\hline USNV & 144 & JUMBO MINE \\
\hline USNV & 57 & KENNEDY DISTRICT \\
\hline USNV & 121 & KINGSTON DISTRICT \\
\hline USNV & 172 & KLONDYKE DISTRICT \\
\hline
\end{tabular}

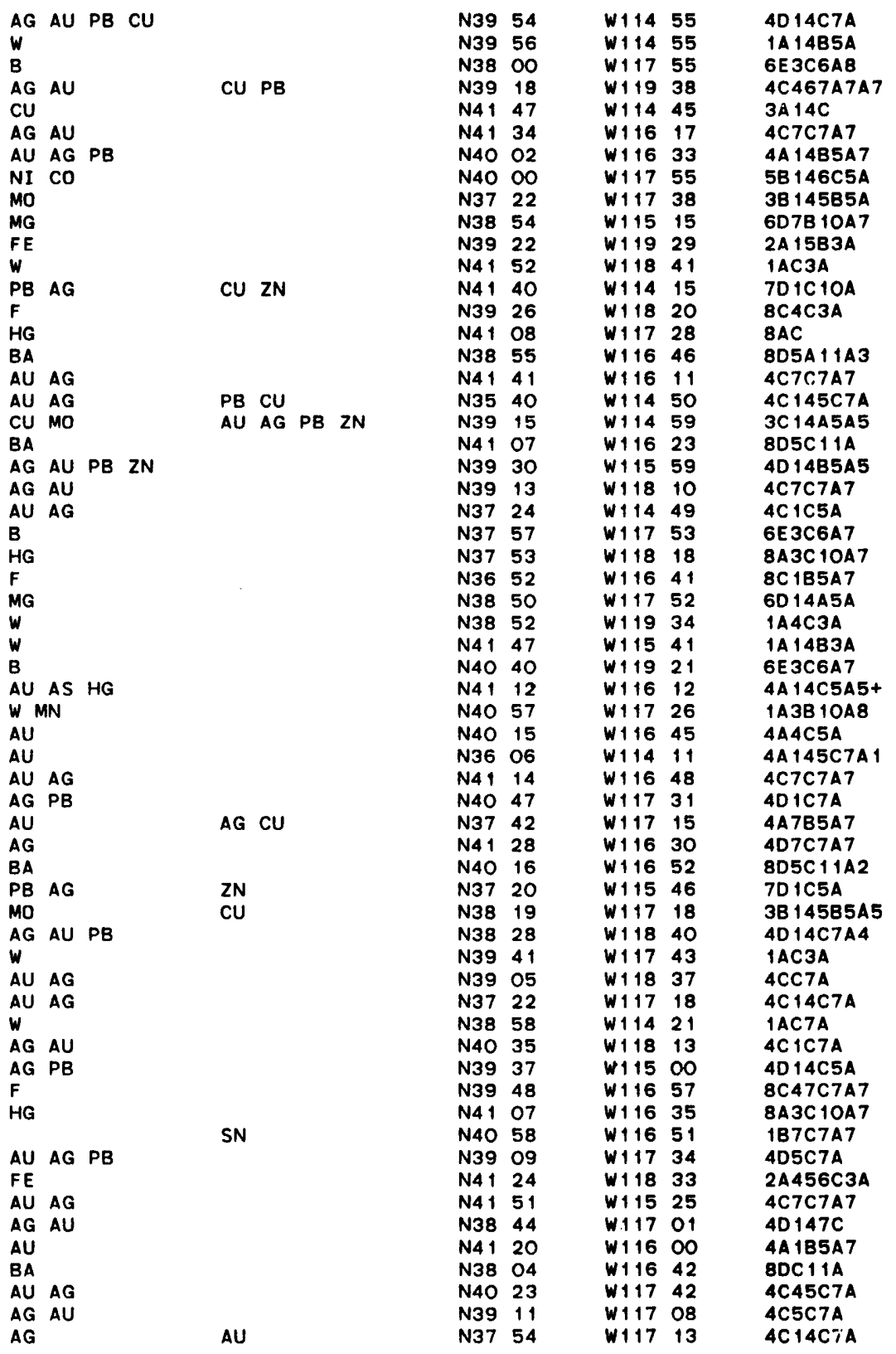




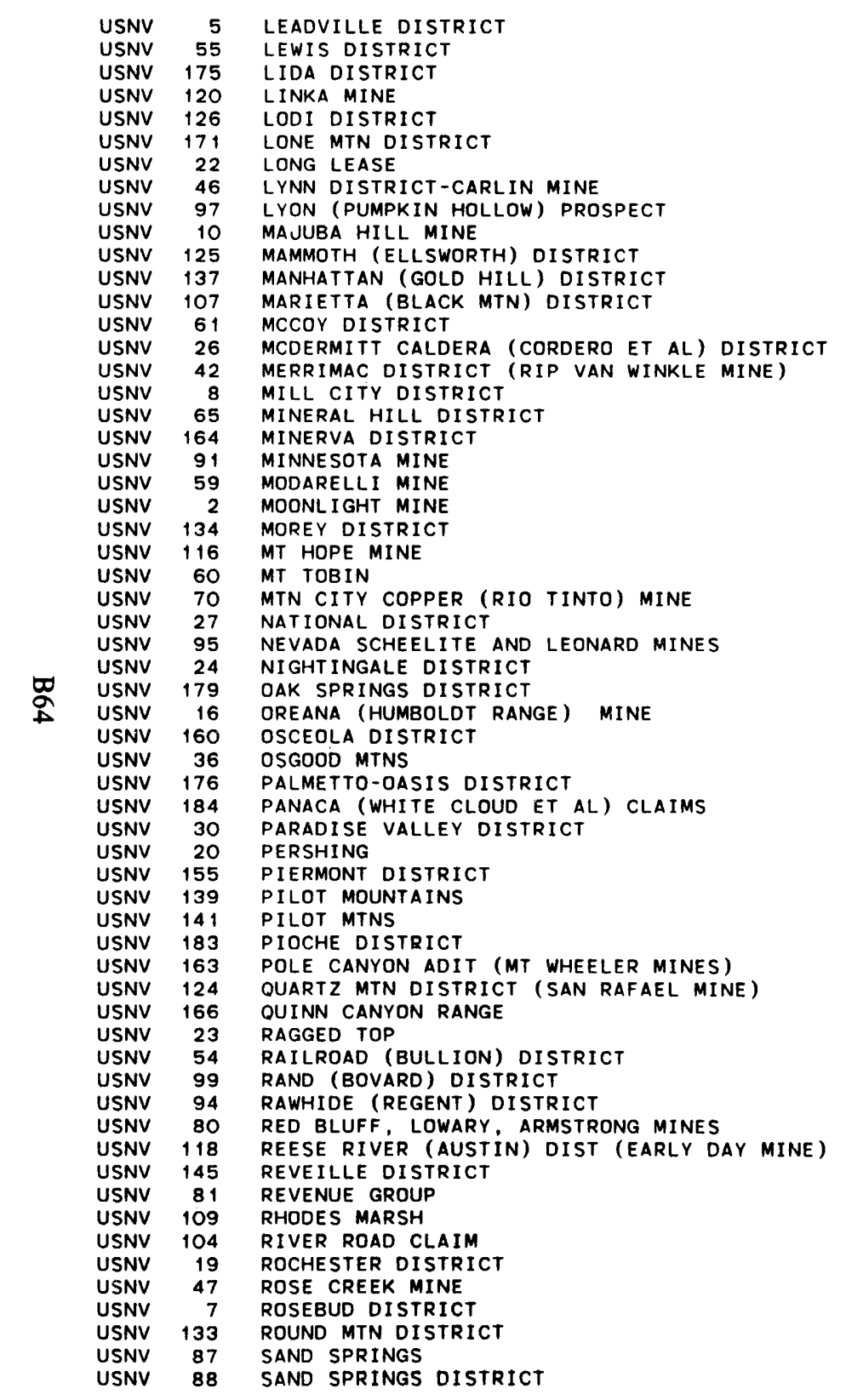

\begin{tabular}{|c|c|c|c|c|c|c|c|c|}
\hline $\mathbf{A G}$ & PB & $\mathrm{ZN}$ & & N4 1 & 07 & $W 119$ & 25 & 407C7A7 \\
\hline$A G$ & PB & & & N4O & 27 & W116 & 52 & 4D45C7A \\
\hline$A G$ & $A U$ & PB & & N37 & 27 & $W 117$ & 33 & 4D14C5A \\
\hline w & & & & N39 & 19 & W116 & 50 & 1AC3A \\
\hline W & & & & N38 & 58 & $W 117$ & 55 & 1A4C7A \\
\hline$A G$ & PB & $\mathrm{ZN}$ & & N37 & 57 & W117 & 25 & 4D 14C5A \\
\hline $\mathbf{w}$ & & & & $\mathrm{N} 4 \mathrm{O}$ & 05 & $W 118$ & 23 & 1ACZA \\
\hline$A U$ & & & & $\mathrm{~N} 4 \mathrm{O}$ & 52 & $W 116$ & 18 & 4A $1 B 5 A 7$ \\
\hline $\mathrm{FE}$ & $\mathrm{CU}$ & & & N38 & 55 & W119 & 05 & 2045A3A4+ \\
\hline SN & $\mathrm{CU}$ & & $\mathbf{u}$ & $\mathrm{N} 4 \mathrm{O}$ & 40 & $W 118$ & 27 & 1B4C3A7 \\
\hline$A U$ & AG & PB ZN & & N38 & 59 & $W 117$ & 45 & 4D5C7A7 \\
\hline$A U$ & $A G$ & & & N38 & 33 & $W 117$ & 03 & 4C45C7A7 \\
\hline$\Delta G$ & PB & & & N38 & 17 & $W 118$ & 22 & 4D5C7A \\
\hline $\mathrm{FE}$ & & & & N4O & 18 & $W 117$ & 12 & $2 A 14 C 3 A$ \\
\hline HG & $u$ & & & N4 1 & 55 & $W 117$ & 47 & 8АЗА5A7 \\
\hline PB & $\mathbf{A G}$ & $\mathrm{ZN}$ & & N4 1 & 07 & $w 116$ & $\infty$ & $7 D 14 C 7 A$ \\
\hline$w$ & & & & N4O & 47 & $W 118$ & 08 & 1A45A3A6 - \\
\hline PB & $\mathbf{A G}$ & & & N4O & 09 & W116 & 06 & $701 C 5 A$ \\
\hline w & & & & N38 & 48 & W114 & 21 & AA $14 B 7 A$ \\
\hline FE & & & $\mathrm{cu}$ & N39 & 03 & $W 119$ & 20 & 2D45C3A4 \\
\hline $\mathrm{FE}$ & & & & N4O & 22 & W116 & 16 & 2A47C5A7 \\
\hline $\mathbf{u}$ & & & & N41 & 47 & $W 118$ & 10 & 9A7C7A6+ \\
\hline $\mathbf{A G}$ & $A U$ & r $B$ MN & & N38 & 40 & $W 116$ & 16 & 4D7C7A7 \\
\hline $\mathbf{Z N}$ & $\mathbf{A G}$ & & $C D$ & N39 & 47 & W116 & 10 & $7014 C 3 A$ \\
\hline HG & & & & N4O & 21 & W117 & 32 & $B A C$ \\
\hline $\mathrm{Cu}$ & & & & N4 1 & 50 & W115 & 59 & $3 A 5 B 11 A 2$ \\
\hline$A U$ & $\mathbf{A G}$ & & SB HG & N4 1 & 50 & W117 & 34 & 4C7C7A7 \\
\hline w & & & & N39 & 02 & W118 & 19 & $1 A 45 B 3 A$ \\
\hline w & & & & $\mathrm{N} 4 \mathrm{O}$ & 01 & W119 & 14 & 1A45C3A \\
\hline $\mathbf{W}$ & & & & N37 & 14 & $W 116$ & 03 & 1A 1C3A \\
\hline w & & & $B E$ & $\mathrm{~N} 4 \mathrm{O}$ & 24 & $W 118$ & 15 & 1AC $1 A$ \\
\hline$A U$ & $A G$ & & & N39 & 04 & W114 & 23 & 4C 14C7A \\
\hline $\mathbf{w}$ & мO & & & N4 1 & 13 & W117 & 15 & 1А45B3A \\
\hline TLC & & & & N37 & 26 & W117 & $43 *$ & IOE $1 \mathrm{C} 1 \mathrm{1B}$ \\
\hline$u$ & & & & N37 & 48 & W114 & 22 & 9A3C 1OA7+ \\
\hline$A U$ & $A G$ & & & N41 & 36 & $W 117$ & 27 & $4 C 5 C 7 A$ \\
\hline SB & & & & N4O & 13 & W118 & 16 & 8BC7A \\
\hline AU & $\mathbf{A G}$ & & & N39 & 30 & $W 114$ & 35 & $4 C+C 7 A$ \\
\hline $\mathbf{w}$ & & & & N38 & 20 & $w 117$ & 53 & 1ACBA \\
\hline HG & & & & N38 & 18 & W117 & 55 & BAC \\
\hline $\mathrm{ZN}$ & PB & $\triangle G C U$ MN & & N37 & 55 & W114 & 29 & $701485 A 5$ \\
\hline $\mathbf{w}$ & & & $\mathrm{BE}$ & N38 & 54 & W114 & 20 & IAIC7A \\
\hline $\mathbf{A G}$ & PB & $\mathrm{ZN}$ & & N39 & 03 & $W 117$ & 58 & 40 14C7A7 \\
\hline $\mathrm{F}$ & & & $A G A U$ & N38 & 09 & $W 115$ & 40 & 8C $1 B 5 A 7$ \\
\hline w & & & & N4O & 03 & $W 118$ & 48 & 1ACZA \\
\hline $\mathbf{A G}$ & PB & cu & & N4O & 30 & $W 116$ & $\infty$ & $4014 C 3 A$ \\
\hline$A U$ & $\mathbf{A G}$ & & CU PB & N38 & 48 & W118 & 24 & 4C7C7A6+ \\
\hline$A U$ & $A G$ & & & N39 & 02 & W118 & 25 & 4C7C7A7 \\
\hline u & & & & N39 & 52 & $W 119$ & 40 & 9AC7A7 \\
\hline $\bar{A} G$ & $A U$ & & u & N39 & 28 & $W 117$ & 04 & $4 C 145 B 7 A 6+$ \\
\hline $\mathrm{ZN}$ & PB & $A G$ & SB HG & N38 & 01 & W116 & 06 & $701 C 5 A 6+$ \\
\hline$\vec{F}$ & & & & N39 & 42 & W118 & 15 & 8C1C7A7 \\
\hline B & & & & N38 & 14 & $W 1+8$ & 02 & 6E $3 C 6 A 8$ \\
\hline$u$ & & & & N38 & 29 & $W 118$ & 54 & 9AC7A \\
\hline $\mathbf{A G}$ & $A U$ & & PB CU & N4O & 18 & $W 118$ & 11 & $4 C 5 C 7 A$ \\
\hline $\mathbf{w}$ & & & & N4O & 51 & $W 117$ & 51 & 1ACBA \\
\hline $\mathbf{A} \mathbf{G}$ & & & CU PB & N4O & 48 & W118 & 36 & 4D7C5A7 \\
\hline$A U$ & $A G$ & & & N38 & 41 & $W 117$ & 04 & 4C7C7A7 \\
\hline B & & & & N39 & 17 & $W 118$ & 25 & 6E3C6A \\
\hline W & & & & N39 & 16 & $W 118$ & 21 & IACBA \\
\hline
\end{tabular}




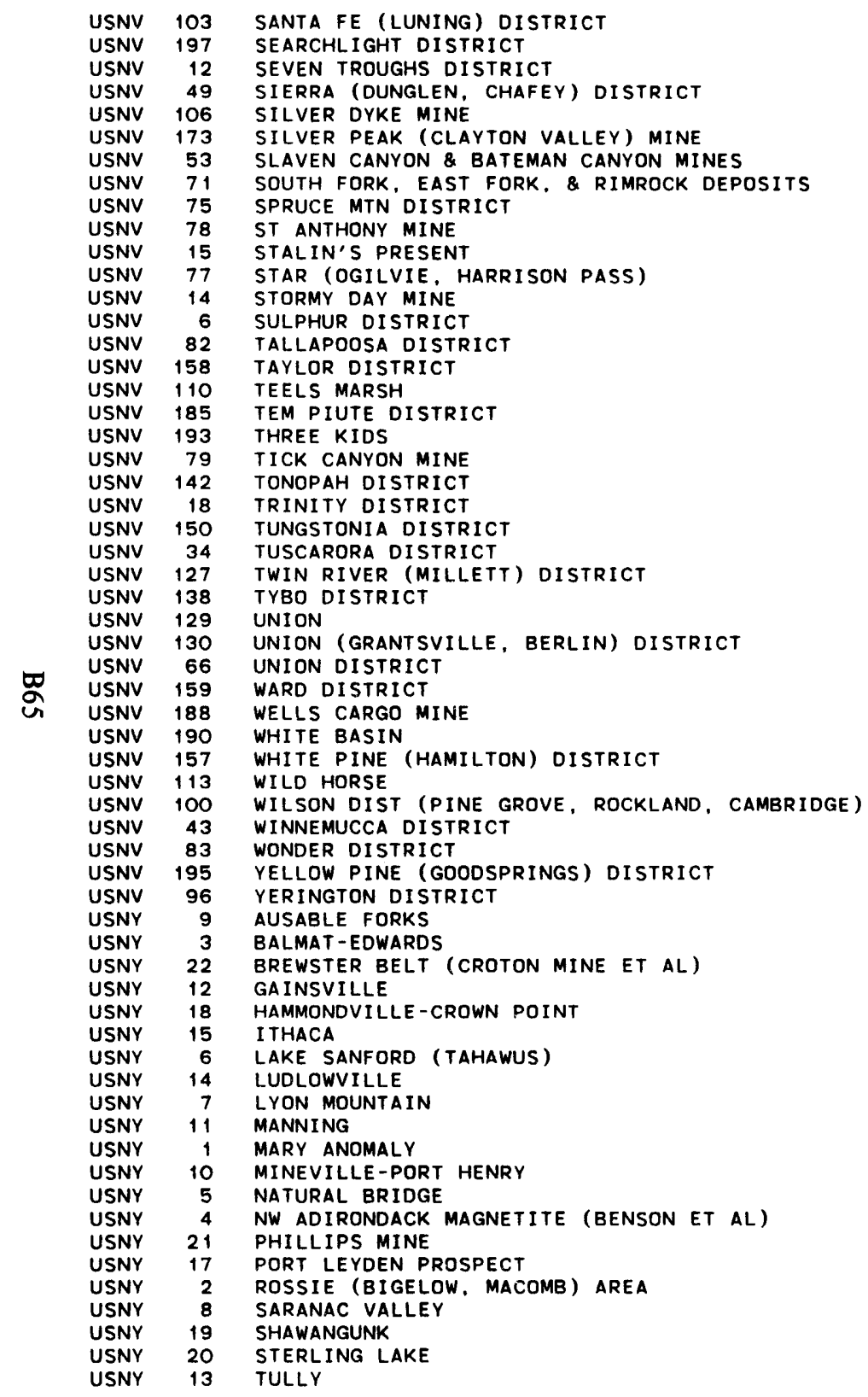

\begin{tabular}{|c|c|c|c|c|c|c|c|c|c|c|}
\hline CU & PB & $A U$ & $\mathbf{A G}$ & SB & & N38 & 30 & W118 & 05 & 3A 14C3A5+ \\
\hline AU & 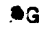 & & & PB & CU & N35 & 27 & W114 & 55 & $4 C 47 C 7 A 6$ \\
\hline$A U$ & $A G$ & & & & & N4O & 30 & $W 118$ & 38 & 4C7C7A7 \\
\hline AU & $A G$ & & & & & N4O & 43 & $W 117$ & 52 & $4 C 6 C 7 A$ \\
\hline$w$ & & & & & & N38 & 19 & $W 118$ & 12 & AA7C7A7 \\
\hline LI & & & & & & N37 & 46 & $w+17$ & 34 & IE $3 A 6 A B$ \\
\hline BA & & & & & & $\mathrm{N} 4 \mathrm{O}$ & 30 & $W 116$ & 48 & $805 C 11 A$ \\
\hline u & & & & & & N41 & 50 & $w 115$ & 52 & 9A7C $10 A$ \\
\hline PB & $A G$ & $Z N$ & & & & N4O & 34 & $W 114$ & 50 & 7D 14C5A \\
\hline $\mathbf{w}$ & & & & & & N39 & 59 & $W 118$ & 42 & 1AC3A \\
\hline $\mathbf{u}$ & & & & & & $\mathrm{N} 4 \mathrm{O}$ & 25 & $W 118$ & 12 & $9 A C 7 A$ \\
\hline w & & & & & & N4O & 19 & $W 115$ & 28 & 1AC3A \\
\hline $\mathbf{w}$ & & & & & & $\mathrm{N} 4 \mathrm{O}$ & 25 & W119 & 17 & 1AC3A \\
\hline AG & PB & & & HG & & N40 & 54 & $W 118$ & 39 & 4D7C5A7 \\
\hline AU & $A G$ & & & & & N39 & 28 & $W 119$ & 15 & 4C7C7A \\
\hline AU & $A G$ & & & & & N39 & 05 & W114 & 40 & 4C 14C7A \\
\hline B & & & & & & N38 & 09 & $W 118$ & 17 & 6E 3C6A8- \\
\hline $\mathbf{w}$ & $A G$ & $\mathbf{F}$ & & & & N37 & 39 & W115 & 37 & 1A 14B3A \\
\hline MN & & & & & & N36 & 04 & W114 & 53 & 1OA3B 1OA7 \\
\hline$u$ & & & & & & N39 & 54 & $W 119$ & 51 & 9A7C7A7 \\
\hline AG & $A U$ & PB & & $u$ & $W S E$ & N38 & 05 & $W 117$ & 14 & 4C7B7A7 \\
\hline AG & PB & & & SN & $A U C U W$ & N4O & 20 & $W 118$ & 30 & 4D4C7A5 \\
\hline w & & & & & & N39 & 40 & $w 114$ & 10 & 1AC7A \\
\hline AG & $A U$ & & & & & N41 & 18 & $w 116$ & 15 & 4C7C7A7 \\
\hline AG & PB & $Z N$ & & & & N38 & 55 & W117 & 16 & 4DC7A \\
\hline$A G$ & $A U$ & PB 2 & $Z N$ & & & N38 & 22 & $w 116$ & 24 & 404C5A7 \\
\hline HG & & & & & & N38 & 54 & W117 & 31 & $8 A C$ \\
\hline$A G$ & PB & $\mathrm{ZN}$ & & & & N38 & 53 & W117 & 35 & 4D5C7A \\
\hline PB & $A G$ & & & & & N4O & 03 & $W 116$ & C3 & $701 C 5 A$ \\
\hline$A G$ & PB & $Z N A$ & $A U C U$ & & & N39 & 04 & W114 & 52 & 4D 14C7A \\
\hline $\mathbf{F}$ & & & & & & N37 & 13 & W114 & 17 & $8 C 1 C 5 A$ \\
\hline B & & & & & & N36 & 20 & W114 & 34 & 6E $3 B 6 A 7$ \\
\hline AG & PB & & & $Z N$ & & N39 & 12 & W115 & 30 & 4D14B5A5 \\
\hline HG & & & & & & N39 & 51 & W117 & 24 & BAC \\
\hline$A U$ & $\mathbf{A G}$ & & & CU & PB ZN & N38 & 40 & W119 & 07 & $4 C 14 C 5 A$ \\
\hline PB & $\mathrm{ZN}$ & AG & & & & N4 1 & 03 & $W 117$ & 42 & 7D5C7A \\
\hline AG & $A U$ & & & & & N39 & 27 & $W 118$ & 02 & 4C7C7A7 \\
\hline $\mathbf{Z N}$ & PB & $A G A$ & $A U C U$ & & & N35 & 52 & W115 & 31 & $701485 A 6+$ \\
\hline $\mathrm{CU}$ & & & & $\mathbf{A G}$ & FE & N38 & 59 & $W 119$ & 11 & 3А45B5A4 \\
\hline FE & & & & & & N44 & 28 & W073 & 40 & $2 A 15 C 2 A 2$ \\
\hline $\mathrm{ZN}$ & PB & & & AG & HG & N44 & 18 & W075 & 20 & $7 C 1 A 11 A 2-4$ \\
\hline FE & $\mathrm{CU}$ & & & & & N4O & 24 & W073 & 40 & $2015 \mathrm{C}$ \\
\hline HAL & & & & & & N42 & 39 & W078 & 06 & $6837-6 \times 3$ \\
\hline $\mathrm{FE}$ & & & & & & N43 & 57 & W073 & 34 & $2 A 15 C 2 A 2$ \\
\hline HAL & & & & & & N42 & 28 & W076 & 34 & $6837-6 \times 3$ \\
\hline $\mathrm{FE}$ & $T I$ & & & $v$ & & N44 & 01 & W074 & 01 & 2C68A5A3 \\
\hline HAL & & & & & & N42 & 34 & W076 & 34 & $6837-6 \times 3$ \\
\hline FE & & & & & & N44 & 43 & W073 & 55 & $2 A 15 B 2 A 2$ \\
\hline $\mathrm{ZN}$ & & & & PB & & N43 & 11 & W078 & 07 & $7 B 3 C 2 A 8$ \\
\hline FE & & & & & & N44 & 36 & W074 & 20 & $2 A 15 C 2 A 2$ \\
\hline $\mathrm{FE}$ & & & & & & N44 & 06 & W073 & 32 & 2A 15B2A2 \\
\hline TLC & & & & & & N44 & 05 & W075 & 24 & IOE IC 1 TA4 \\
\hline $\mathrm{FE}$ & & & & & & N44 & 10 & W074 & 56 & 2A15A2A2 \\
\hline FES & & & & CU & NI U & N41 & 19 & W073 & 58 & $11 B 1456 C 11 A 1$ \\
\hline & & & & TI & & N43 & 35 & W075 & 19 & $2 B 3 B 4 A 8$ \\
\hline PB & $Z N$ & & & BA & $F$ & N44 & 25 & WO75 & 38 & 7C1C7A5t \\
\hline $\mathrm{FE}$ & & & & & & N44 & 37 & W073 & 50 & $2 A 15 C 2 A 2$ \\
\hline PB & $2 \mathrm{~N}$ & & & $\mathrm{CU}$ & & N41 & 37 & W074 & 26 & 7C1C7A4t \\
\hline $\mathrm{FE}$ & & & & & & N41 & 12 & W074 & 16 & $2 A C$ \\
\hline HAL & & & & & & N42 & 48 & W076 & 07 & $6 B 37-6 \times 3$ \\
\hline
\end{tabular}




\begin{tabular}{|c|c|c|c|c|c|c|c|c|c|}
\hline USNY & 16 & WATKINS & HAL & & N42 & 25 & W076 & 53 & $6837-6 \times 3$ \\
\hline USOH & 5 & AKRON (KENMORE) & HAL & & N4 1 & 03 & W081 & 32 & $6837-6 \times 3$ \\
\hline USOH & 6 & BARBERTON & HAL & & N4O & 58 & W081 & 34 & $6837-6 \times 3$ \\
\hline USOH & 4 & CLEVELAND & HAL & & N4 1 & 29 & W08 1 & 40 & $6837-6 \times 3$ \\
\hline USOH & 3 & FAIRPORT HARBOR & HAL & & N41 & 45 & W08 1 & 15 & $6 B 37-6 \times 3$ \\
\hline USOH & 2 & OTTAWA COUNTY & GYP & & N4 1 & 30 & W082 & 54 & 6A37B6A3 \\
\hline USOH & 7 & RITTMAN & HAL & & $\mathrm{N} 4 \mathrm{O}$ & 52 & W08 1 & 45 & $6837-6 \times 3$ \\
\hline USOH & 1 & TOLEDO & GYP & & N41 & 41 & W083 & 45 & $6 A 37 C$ \\
\hline USOK & 7 & BLAINE COUNTY & GYP & & N35 & 46 & W098 & 14 & 6A37C6A4 \\
\hline USOK & 6 & BUCHER MINE & GYP & & N35 & 56 & w098 & 23 & 6A37C6A4 \\
\hline USOK & 18 & BYARS AND RANDLETT PROPERTIES & $\mathrm{u}$ & & N34 & 06 & wo98 & 31 & $9 A 37 C 10 A 4+$ \\
\hline USOK & 15 & CEMENT DEPOSIT & $u$ & $\mathbf{v}$ & N34 & 54 & W098 & 08 & $9437 C_{10 A 4+}$ \\
\hline USOK & 16 & CRETA MINE & $\mathrm{cu}$ & AG & N34 & 29 & Wo99 & 34 & 3A37B 10A4 \\
\hline USOK & 20 & DAVIS DISTRICT & $\mathbf{Z N}$ & $C D$ & N34 & 27 & W097 & 12 & $7837 C_{10 A 3+}$ \\
\hline USOK & 10 & ELM RIVER AREA & GYP & & N34 & 55 & wo99 & 54 & 6A37C6A4 \\
\hline USOK & 11 & HARMON COUNTY & HAL & & N34 & 51 & w099 & 54 & $6837-6 \times 4$ \\
\hline USOK & 14 & LAKE LAWTONKA & TI & & N34 & 43 & w098 & 31 & $2 C C 4 A 8$ \\
\hline USOK & 4 & LEE UTO PROSPECT & $\mathrm{cu}$ & & N36 & 21 & W096 & 55 & 3АЗ 7 C $10 A 4$ \\
\hline USOK & 12 & MANGUM PROSPECT & $\mathrm{cu}$ & & N34 & 46 & wo9g & 32 & $3 A 37 C_{10 A 4}$ \\
\hline USOK & 17 & MATHIAS AND OBERLANDER PROPERTIES & $u$ & cu & N34 & 28 & W098 & 49 & 9A37C 1OA4+ \\
\hline USOK & 21 & OUACHITA MOUANTAINS & MN & & N34 & 15 & w094 & $35 *$ & 1OAC2B3 \\
\hline USOK & 19 & PAOLI PROSPECT & $\mathrm{CU}$ & & N34 & 48 & w097 & 22 & 3A37C $10 A 4$ \\
\hline USOK & 9 & ROGER MILLS COUNTY & HAL & & N35 & 13 & W099 & 38 & $6837-6 \times 4$ \\
\hline USOK & 3 & SOUTHARD AND WEST OF OKENE MINES & GYP & & N36 & 08 & W098 & 30 & 6A37B6A4 \\
\hline USOK & 5 & TRI-STATE DISTRICT & $\mathrm{ZN}$ PB & $C D$ & N36 & 58 & W094 & 38 * & 7C37A 10B4t \\
\hline USOK & 8 & WEATHERSFORD & GYP & & N35 & 32 & W098 & 46 & 6A37C6A4 \\
\hline USOK & 13 & WITCHITA MOUNTAINS & FE TI & & N34 & 44 & W098 & 48 & 2C68C5A2 \\
\hline USOK & 1 & WOODS COUNTY & HAL & & N36 & 49 & Wo99 & 16 & $6 \mathrm{~B} 37-6 \times 4$ \\
\hline USOK & 2 & WOODWARD COUNTY & GYP & & N36 & 30 & w099 & 05 & 6A37C6A4 \\
\hline USOR & 44 & ASHLAND MINE & AU & & N42 & 08 & W122 & 40 & $4 A 15 C 7 A$ \\
\hline USOR & 45 & BEAR CREEK & $u$ & & N43 & 59 & $w 120$ & 44 & $9 A 34 C 5 A 7$ \\
\hline USOR & 9 & BEAR CREEK DISTRICT & HG & & N44 & 01 & W120 & 43 & $8 A 7 C$ \\
\hline USOR & 26 & BLACK BUTTE & HG & & N43 & 33 & $W 123$ & 01 & 8А7B5A7 \\
\hline USOR & 25 & BOHEMIA DISTRICT (CHAMPION ET AL) & PB CU ZN & AU AG & N43 & 35 & W122 & 31 & 70467C7A7 \\
\hline USOR & 27 & BONANZA-NONPAREIL MINES & HG & & N43 & 23 & $W 123$ & 10 & 8АЗ7B5A7 \\
\hline USOR & 50 & BRETZ OPALITE & HG & & N42 & 02 & W117 & 56 & 8АЗB 10A7 \\
\hline USOR & 39 & CHOLLARD (GOLCONDA) & CR & & N42 & 05 & W123 & 33 & $5458 \mathrm{C}$ \\
\hline USOR & 20 & COOS BAY AREA (S. SLOUGH, SEVEN DEVILS ET AL) & CR TI & & N43 & 15 & $\mathbf{W 1 2 4}$ & 21 & $5 A 37 B 4 A 8$ \\
\hline USOR & 15 & CORNUCOPIA MINE & AU & U ZN PB & N44 & 59 & $w+17$ & 11 & $4 A 45 C 7 A 5+$ \\
\hline USOR & 19 & COYOTE & SB & AU & N44 & 18 & $W 117$ & 44 & $8 B C$ \\
\hline USOR & 12 & EASTERN BLUE MOUNTAINS REGION & AU & AG & N44 & 47 & $W 118$ & $18 *$ & $4 A 3 B 4 B 8$ \\
\hline USOR & 37 & EIGHT DOLLAR MOUNTAIN & NI & & N42 & 14 & W123 & 38 & $5858 \mathrm{C9A7+}$ \\
\hline USOR & 32 & EVANS CREEK AREA & CR & & N42 & 38 & W123 & 13 & 5A58C5A \\
\hline USOR & 34 & GALICE DISTRICT (ALMEDA, BENTON ET AL) & AU & CU AG PB & N42 & 33 & W123 & 34 & $4045 C 7 A 4+$ \\
\hline USOR & 30 & GALICE DISTRICT PLACERS & AU & & N42 & 38 & W123 & 39* & $4 A 3 C 4 B 7+$ \\
\hline USOR & 23 & GOLD BEACH & AU & CR PT & N42 & 25 & W124 & 24 & $4 A 37 C 4 A B$ \\
\hline USOR & 18 & GRAY EAGLE (KOEHLER) & SB & AU & N44 & 47 & W117 & 45 & 8B5C7A \\
\hline USOR & 31 & GREENBACK MINE ET AL & $A U$ & & N42 & 40 & $w 123$ & 17 & 4A5C7A \\
\hline USOR & 7 & HORSE HEAVEN MINE & HG & & N44 & 40 & W120 & 35 & 8A7B5A7 \\
\hline USOR & 16 & IRON DYKE (HOMESTEAD) MINE & $\mathrm{CU}$ & AU AG & N45 & 02 & W116 & 52 & $3 A 5 C 11 A 3+$ \\
\hline USOR & 14 & JOHN DAY (CANYON MOUNTAIN) DISTRICT & CR & & N44 & 21 & $w 118$ & 52* & 5А58B5B3 \\
\hline USOR & 17 & KEATING (BALM CREEK) MINE & $\mathrm{CU}$ & & N44 & 55 & $w 117$ & 20 & $3 A 5 C 11 A 3+$ \\
\hline USOR & 49 & LAKE ALVORD & B & & N42 & 20 & $w 118$ & 40 & GE3C6A8 \\
\hline USOR & 21 & LOWER ROGUE RIVER AREA & $C R$ & & N42 & 39 & W124 & $04 *$ & $5 A 58 \mathrm{C} 5 \mathrm{~B}$ \\
\hline USOR & 10 & MAURY MOUNTAIN DISTRICT & HG & & N44 & 05 & $w 120$ & 25 & BATC \\
\hline USOR & 33 & MEADOWS DISTRICT (WAR EAGLE ET AL) & HG & & N42 & 38 & W122 & 58 & 8A15C7A7 \\
\hline USOR & 43 & MOUNT ASHLAND AREA & CR & & $\mathrm{N} 42$ & 09 & $w 122$ & 48 & 5A58C5A \\
\hline USOR & 4 & NORTH SANTIAM DISTRICT & $Z N P B C U$ & $A G A U$ & N44 & 51 & W122 & 11 & $70467 C 7 A 7$ \\
\hline USOR & 5 & OAK GROVE DISTRICT & HG & & N45 & 04 & $w 121$ & 58 & 8А7C7A7 \\
\hline USOR & 8 & OCHOCO DISTRICT & HG & & N44 & 24 & $W 120$ & 30 & $8 A 7 C$ \\
\hline
\end{tabular}




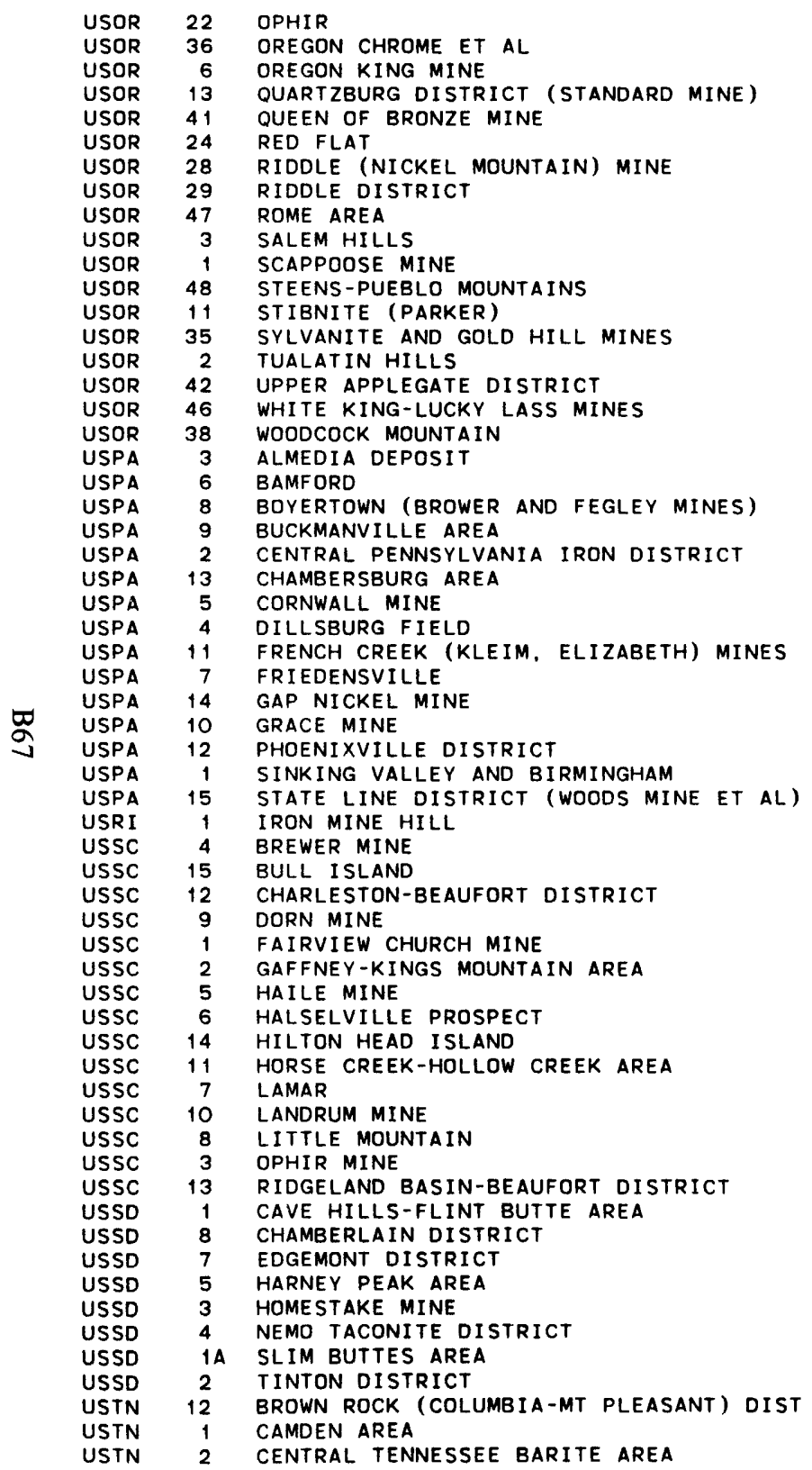

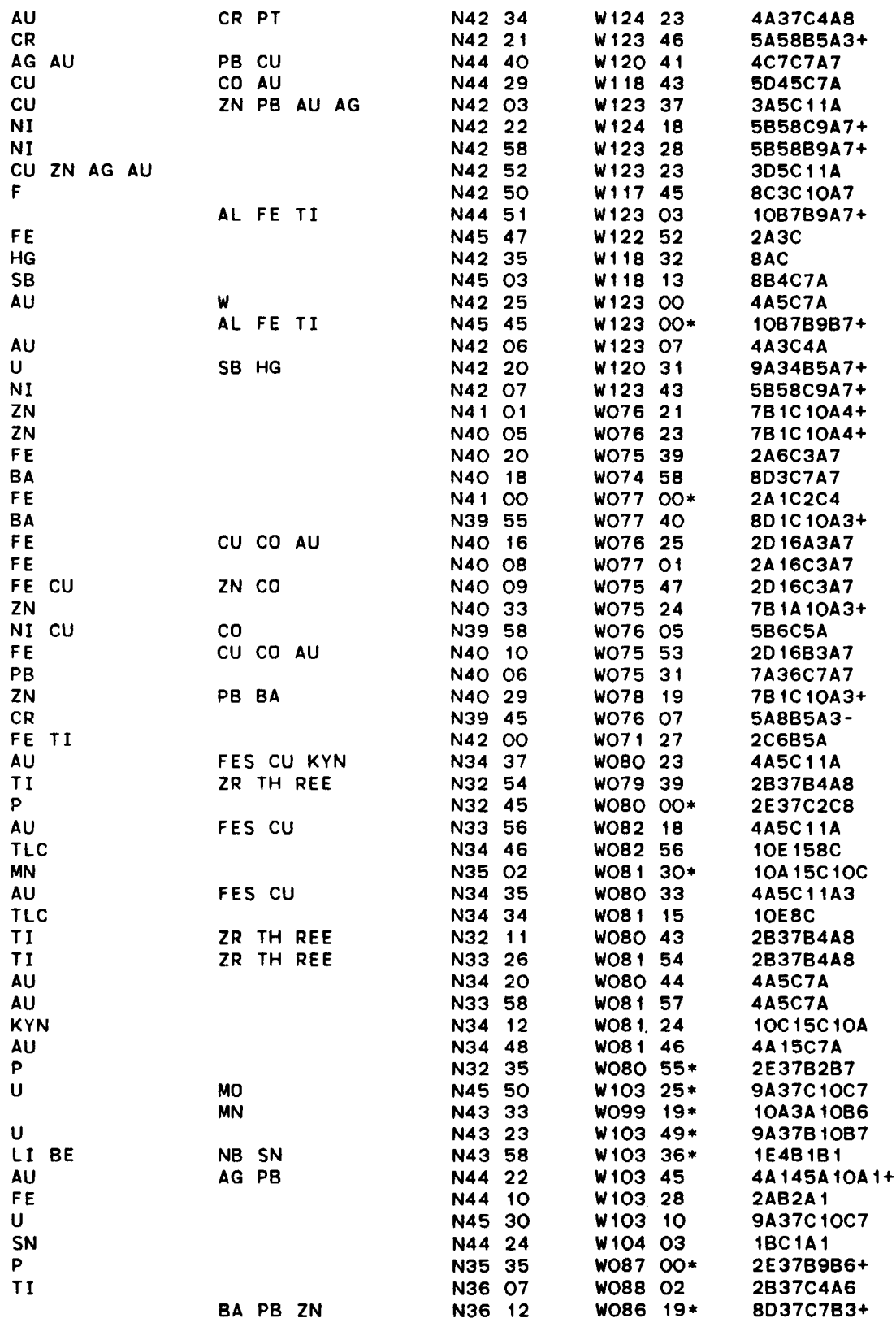




\begin{tabular}{|c|c|c|c|}
\hline & USTN & 15 & COKER CREEK DISTRICT \\
\hline & USTN & 5 & COPPER RIDGE-FLAT GAP DISTRICT \\
\hline & USTN & 16 & DUCKTOWN DISTRICT \\
\hline & USTN & 8 & EAST TENNESSEE MANGANESE DISTRICTS \\
\hline & USTN & 3 & ELMWOOD MINE \\
\hline & USTN & 7 & EMBREEVILLE \\
\hline & USTN & 10 & LEXINGTON-PINEY CREEK AREA \\
\hline & USTN & 6 & MASCOT-JEFFERSON CITY-NEW MARKET DIST \\
\hline & USTN & 9 & NATCHEZ TRACE STATE PARK AREA \\
\hline & USTN & 4 & NEW PROSPECT-STRAIGHT CREEK MINES \\
\hline & USTN & 13 & ROCKWOOD-CARDIFF DISTRICT \\
\hline & USTN & 14 & SWEETWATER DISTRICT \\
\hline & USTN & 11 & WESTERN HIGHLAND RIM DISTRICT \\
\hline & USTX & 3 & ACME MINE \\
\hline & USTX & 16 & ALLAMOORE COPPER DISTRICT \\
\hline & USTX & 18 & ALLAMOORE TALC DISTRICT \\
\hline & USTX & 5 & BAILEY COUNTY \\
\hline & USTX & 39 & BIG CREEK MINE \\
\hline & USTX & 36 & BOLING DOME \\
\hline & USTX & 44 & BRYAN MOUND \\
\hline & USTX & 15 & CAVE PEAK \\
\hline & USTX & $20 A$ & CHRISTOVAL WEST AREA \\
\hline & USTX & 43 & CLEMENS DOME \\
\hline & USTX & 48 & FALFURRIAS MINE \\
\hline & USTX & 38 & FANNETT DOME \\
\hline & USTX & 20 & FORT STOCKTON AREA \\
\hline & USTX & 13 & FRANKLIN MOUNTAINS \\
\hline & USTX & 24 & FREDERICKSBURG AREA \\
\hline & USTX & 7 & GARZA COUNTY \\
\hline & USTX & 45 & GULF HILL MINE \\
\hline & USTX & 1 & HART-MANSF I ELD RANCH \\
\hline & USTX & 26 & HOCKLEY MINE \\
\hline & USTX & 42 & HOSKINS MOUND \\
\hline & USTX & 31 & KARNES COUNTY AREA \\
\hline & USTX & 49 & KELSEY RANCH \\
\hline & USTX & 23 & LLANO DISTRICT \\
\hline & USTX & 22 & LLANO UPLIFT DISTRICT \\
\hline & USTX & 40 & LONG POINT \\
\hline & USTX & 9 & LONGWORTH MINE \\
\hline & USTX & 30 & MARISCAL \\
\hline & USTX & 33 & MCLEAN AND FELDER MINES \\
\hline & USTX & 21 & MENARD AREA \\
\hline & USTX & 37 & MOSS BLUFF \\
\hline & USTX & 41 & NASH DOME \\
\hline & USTX & 32 & NEW RANCH \\
\hline & USTX & 12 & NORTH BASIN \\
\hline & USTX & 35A & ORCHARD DOME \\
\hline & USTX & 47 & PALANGANA DOME \\
\hline & USTX & 34 & PROSPECT \\
\hline & USTX & 35 & PURSCH RANCH AREA \\
\hline & USTX & 4 & RED BED COPPER ( $N$. CENTRAL TEXAS) AREAS \\
\hline & USTX & 8 & ROTAN MINE \\
\hline & USTX & 14 & RUSTLER SPRINGS (CULBERSON) \\
\hline & USTX & 2 & SAUL RANCH \\
\hline & USTX & 28 & SHAFTER DISTRICT (PRESIDIO MINE ET AL) \\
\hline & USTX & 17 & SIERRA BLANCA DISTRICT \\
\hline & USTX & 25 & SOUTH BASIN \\
\hline & USTX & 27 & SPINDLETOP MINE \\
\hline & USTX & 10 & SWEETWATER MINE \\
\hline & USTX & 29 & TERLINGUA DISTRICT \\
\hline
\end{tabular}

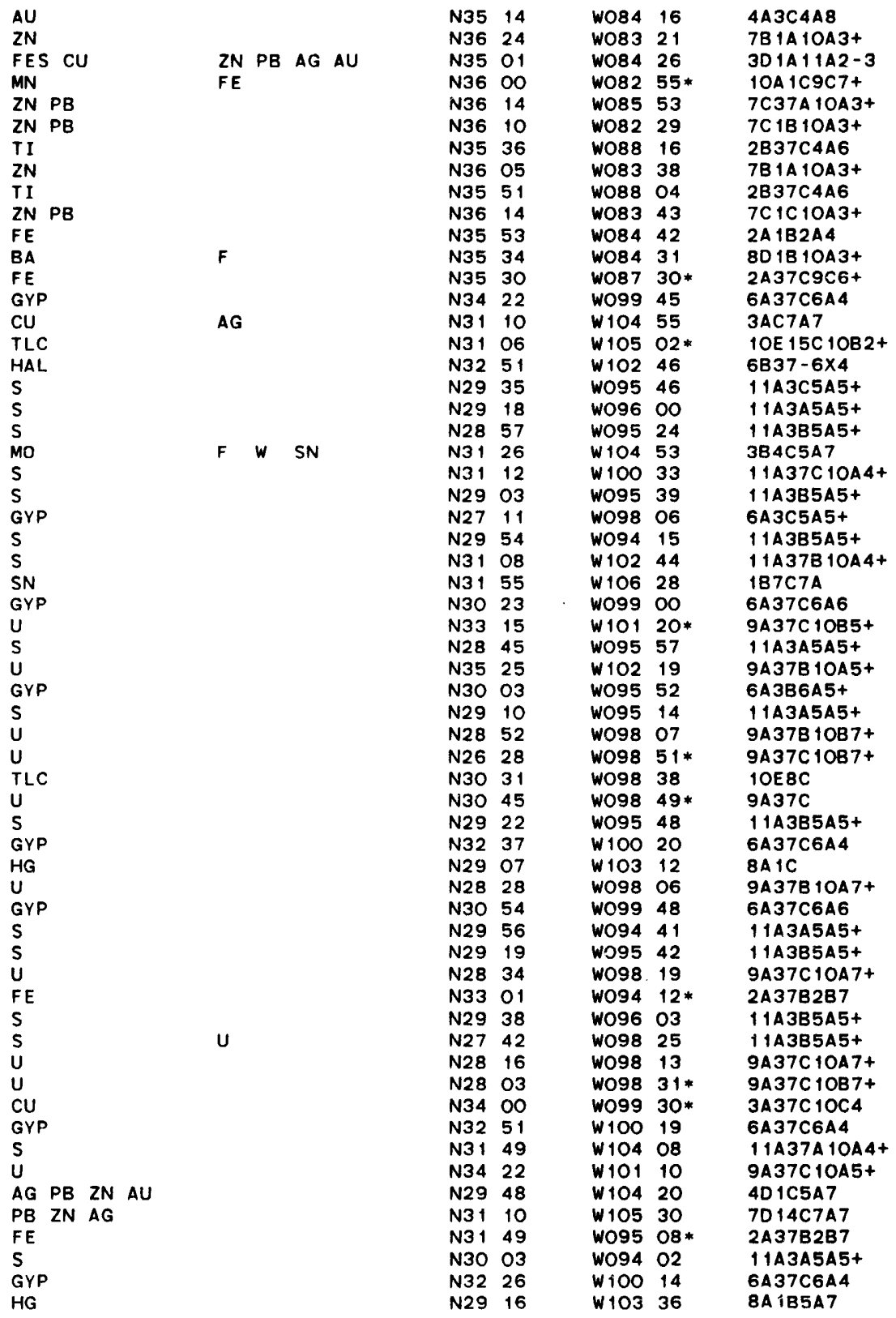


COPPER RANCH BEAVER LAKE DISTRICT-OK DEPOSIT

BINGHAM (WEST MTN) DIST, CARR FORK ET AL BONNEVILLE BASIN

BRADSHAW DISTRICT (CAVE MINE)

BULL VALLEY-COVE MTN DISTRIC

BULLOCK GROUP

CALF MESA AREA
CAMP FLOYD (MERCUR) DISTRICT

CANE CREEK ANTICLINE

CARBONATE DISTRICT (DYER MINE)

CASTLE PEAK DRAW AREA

CAVE CREEK AREA

CIRCLE CLIFFS AREA

COPPER ROCK \& WHITE STAR CLAIMS

COTTONWOOD CANYON AREA

CRAWFORD MOUNTAINS MINE

DEVIL GROUP

DRUM MTNS (DETROIT) DISTRICT

DUGWAY DISTRICT

EAGLE NEST GROUP

ERICKSON DISTRICT

ESCALANTE MINE

\section{GOLD HILL DISTRICT (AND CLIFTON AREA)}

GRAND COUNTY DISTRICT

GREAT SALT LAKE DEPOSITS

GREEN RIVER AREA

GROUSE CREEK RANGE

HENRY MOUNTAINS AREA

HORSE EAR CLAIM

HOUSE RANGE DEPOSITS

KOLOB (SCHOOL SECTION) MINE

LISBON VALLEY \& NEARBY AREAS

LITTLE \& BIG COTTONWOOD DISTRICTS

\section{LUCIN DISTRICT}

\section{MARYSVALE DISTRICT}

MINERAL MOUNTAINS DEPOSITS

MONTEZUMA CANYON AREA

MONUMENT VALLEY AREA
MT NEBO DISTRICT

MT NEBO DISTRICT

MYSTERY SNIFFER AND
NORTH TINTIC DISTRICT

OHIO \& MT BALDY DISTRICTS

\section{OPHIR DISTRICT}

PARADOX SALT BASIN

PARK CITY DISTRICT

PINE GROVE PROSPECT

POLAR MESA AREA

PROMONTORY DISTRICT

ROCK CREEK AREA

SAN FRANCISCO \& PREUSS DISTRICTS

SAN RAFAEL SWELL-GREEN RIVER DESERT AREA

CU

MN

HAL MG $K$ LI B

U $V$

u v

U

FE

$U$

PB ZN AG CU AU

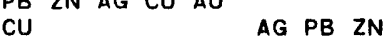

$\mathrm{K} A L U$

AG PB ZN

w $v$

$\begin{array}{ll}U & V \\ P B & A G\end{array}$

$$
\text { PB }
$$

AG PB ZN

$\begin{array}{lll}A G & A U & P B \\ P B & Z N & A G\end{array}$

AU CU

$P B$ ZN AG CU AU

$U$

ZN PB CU

TI

PB AG ZN AU CU MO BA
$\begin{array}{ll}W 104 & 53 \\ w 111 & 36\end{array}$

$W 11136$
$W 111$

$\begin{array}{lll}W & 111 & 53 \\ W & 113 & 50\end{array}$

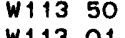

$\begin{array}{ll}W 113 & 08\end{array}$

W112 09

$W 11337 *$

$\begin{array}{lll}W & 11257 \\ W & 113 & 48\end{array}$

$\begin{array}{ll}W 113 & 48 \\ W & 112 \\ W\end{array}$

W110 43

$W 11210$

W109 42*

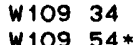

W109 27

W111 09*

W110 57

W109 36

W111 17

W109 31

$\begin{array}{lll}w 113 & 03\end{array}$

$\begin{array}{lll}W 113 & 13\end{array}$

$W 109$
$W 112$

$\begin{array}{ll}W \\ W 1113 & 59 \\ W & 413\end{array}$

W113 27

$W 11349$

$W 11223$

$\begin{array}{ll}W 109 & 53 \\ W 112 & 30\end{array}$

$\begin{array}{lll}W 112 & 30^{*} \\ W & 110 & 20 *\end{array}$

$W 11347$

W1 10 37*

$w 10940$
$w 113$

$\begin{array}{ll}W 11323 \\ w 113 & 13\end{array}$

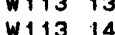

$\begin{array}{lll}W & 113 & 14 \\ w 109 & 11\end{array}$

$W 10911$

$\begin{array}{lll}W & 31 & 38 \\ W & 114 & 00\end{array}$

$W 11400$
$W 11212$

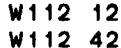

$W 11242$
$W 10914$

W110 18*

$\begin{array}{lll}W & 111 & 47\end{array}$

$W 11234$
$W 112$
$W$

$\begin{array}{ll}W 112 & 10 \\ W & 112\end{array}$

$\begin{array}{ll}W 112 & 18 \\ W & 112 \\ W\end{array}$

W108 00

$W 11129$

$W 11335$
$W 109$

$W 109$
$W 112$

$W 11226$
$W 111$
$W$

W113 16

W110 48*
$6837-6 \times 4$

4B37C5A4+

7D 14C5A7

$8 B 37 \mathrm{C} 10 \mathrm{~A}$

4D 14C5A7

3A 14C3A

C4C5A

3C 14A5A7

6B3A6B8

$2 A 347 C 5 A 7$

9A37C10A6+

9A37C 10A5

4A 14B5A7

3A37C5A4+

9A37C $10 B 6$

9C37C 10A5+

$9 A 37 C 10 B$

9A37C 10A5+

9C37C $10 A$

9A37C10A5+

10A 1C5A

7014C5A7

9A37C 10A5

OAC7A

4D 14C5A

4D 14C3A7

4C47C7A7

1OA37C $10 A 5$

6B3A6B8

9C37B 10B5+

1A 1C $3 A$

9C37C 10B5+

1A $14 B 3 A$

2A347A5A7

9C37A 10A5+

7D 1485A7

3D 14C5A

6C47B5A7

1A 1C3A

9C37C 10A

$701 C 5 A$

9A7B7A7

4D 14C3A7

4D 14C5A7

7D 14B5A7

6B37A6C4

7D 14A5A7

3B 14B5A

9C37C 10A5+

$7014 \mathrm{C}$

2B37C4A6

9 C37B 1OB5+ 


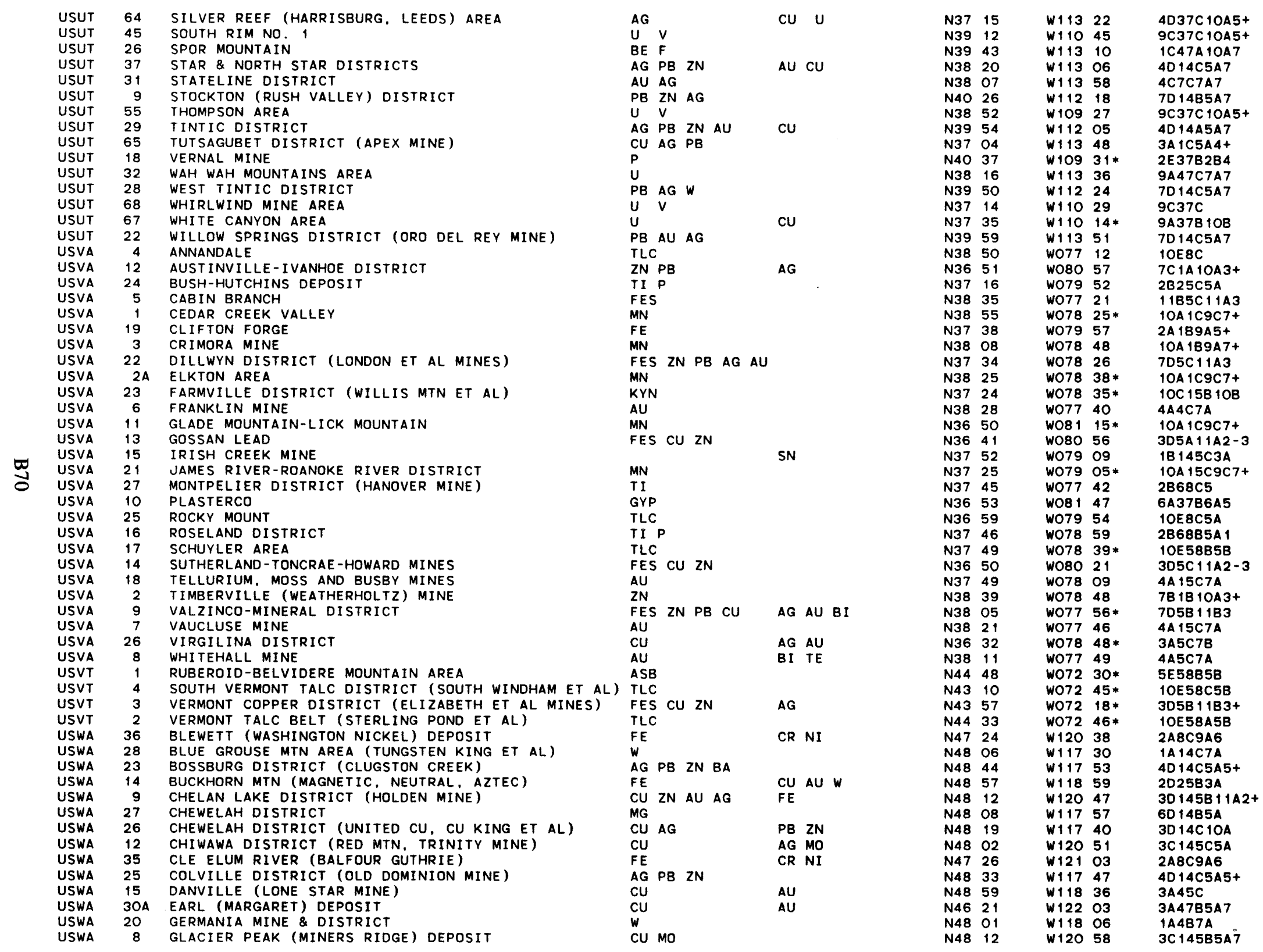




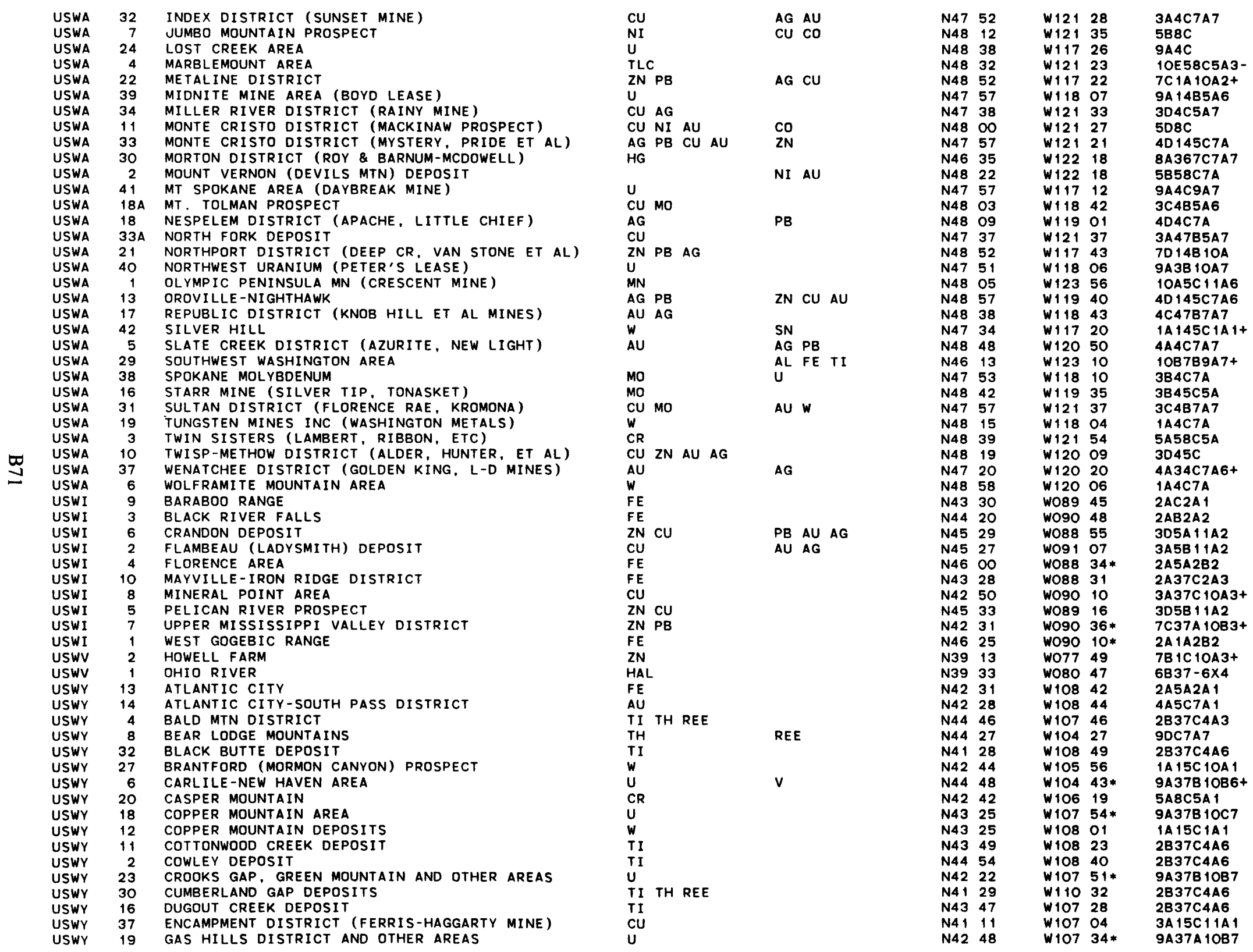




$\begin{array}{lrl}\text { USWY } & 9 & \text { GIBBS CREEK DEPOSITS } \\ \text { USWY } & 10 & \text { GRASS CREEK DEPOSIT } \\ \text { USWY } & 28 & \text { HARTVILLE DISTRICT. SUNRISE MINE } \\ \text { USWY } & 7 & \text { HEETLAND AND OTHER MINES } \\ \text { USWY } & 40 & \text { IRON MOUNTAIN DISTRICT } \\ \text { USWY } & 15 & \text { KAYCEE DISTRICT } \\ \text { USWY } & 35 & \text { KETCHUM BUTTE AND OTHER AREAS } \\ \text { USWY } & 29 & \text { LEEFE MINE } \\ \text { USWY } & 3 & \text { LOVELL DEPOSIT } \\ \text { USWY } & 17 & \text { MUD CREEK DEPOSIT } \\ \text { USWY } & 26 & \text { NEW HOPE AREA } \\ \text { USWY } & 5 & \text { NORTH-CENTRAL WYOMING URANIUM AREAS } \\ \text { USWY } & 38 & \text { PLATT PEGMATITE } \\ \text { USWY } & 36 & \text { POISON BASIN (BAGGS) AREA } \\ \text { USWY } & 1 & \text { PRYOR MTN AREA } \\ \text { USWY } & 24 & \text { PUMPKIN BUTTES AREA } \\ \text { USWY } & 39 & \text { RAMBLER MINE } \\ \text { USWY } & 34 & \text { RED CREEK DEPOSIT } \\ \text { USWY } & 33 & \text { SALT WELLS CREEK DEPOSIT } \\ \text { USWY } & 22 & \text { SEMINOE } \\ \text { USWY } & 21 & \text { SHIRLEY BASIN } \\ \text { USWY } & 25 & \text { SOUTHERN POWDER RIVER BASIN } \\ \text { USWY } & 31 & \text { WESTVACO } \\ \text { VIVI } & 1 & \text { VIRGIN GORDA COPPER MINE } \\ & & \end{array}$

\begin{tabular}{|c|c|c|c|c|c|c|c|}
\hline TI & TH REE & & N43 & 10 & $w 110$ & 28 & 2.B37C4A5 \\
\hline TI & & & N43 & 54 & $W 108$ & 33 & $2 \mathrm{~B} 37 \mathrm{C}$ \\
\hline FE & & & N42 & 19 & $W 104$ & 42 & 2A 1B2A1 \\
\hline $\mathbf{u}$ & & & N44 & 40 & $W 104$ & $05 *$ & $9 A 37 C_{10 C 6+}$ \\
\hline $\mathrm{FE}$ & TI & v & N4 & 36 & W 105 & $16 *$ & 2C68B5B2 \\
\hline u & & & N43 & 45 & $w 106$ & 30 & $9 A 37 \mathrm{C} 10 \mathrm{C} 7$ \\
\hline$u$ & & & N4 & 16 & W 107 & $25 *$ & $9 A 37 C 10 B 7$ \\
\hline $\mathbf{P}$ & & & N4 & 53 & $w 110$ & 58 & 2E37B2A3 \\
\hline TI & TH REE & & N44 & 48 & W 108 & 27 & $2 B 37 C 4 A 6$ \\
\hline TI & & & N43 & 43 & W107 & 45 & $2 B 37 C$ \\
\hline u & & & N42 & 55 & $W 104$ & $50 *$ & $9 A 37 C_{10 C 7}$ \\
\hline u & & & N44 & 30 & W 107 & $51 *$ & $9 A 37 C 10 C$ \\
\hline REE & & & N4 & 06 & $W 106$ & 32 & $11 C 4 C 1 A t$ \\
\hline$u$ & SE & & N4 & 03 & W107 & 46 & 9A37B 1OA7 \\
\hline$u$ & & & N45 & $\infty$ & $W 108$ & $18 *$ & 9A1B5B \\
\hline u & & & N43 & 45 & W105 & $55 *$ & 9A37B $10 B 7$ \\
\hline $\mathrm{cu}$ & & PT $P D \quad A U \quad A G$ & N4 & 15 & $W 106$ & 14 & $3 A 145 C 7 A 1$ \\
\hline TI & & & N4 & 02 & $W 109$ & 14 & $2 B 37 \mathrm{C}$ \\
\hline TI & TH REE & & N4 & 10 & $W 109$ & 02 & $2 B 37 C 4 A 6$ \\
\hline $\mathrm{FE}$ & & & N42 & 10 & W107 & 02 & $2 A 5 C 2 A 1$ \\
\hline$u$ & & & N42 & 19 & W106 & $10 *$ & 9A37A 1OB7 \\
\hline u & & & N43 & 00 & W105 & $32 *$ & 9A37B 10B7 \\
\hline TRO & & & N4 & 25 & W109 & 29 & 6B37A6A7 \\
\hline cu & MO & & N18 & 29 & WO64 & 25 & 3C45C7A6+ \\
\hline
\end{tabular}


